Göttinger Studien zur Kulturanthropologie / Europäischen Ethnologie Göttingen Studies in Cultural Anthropology / European Ethnology

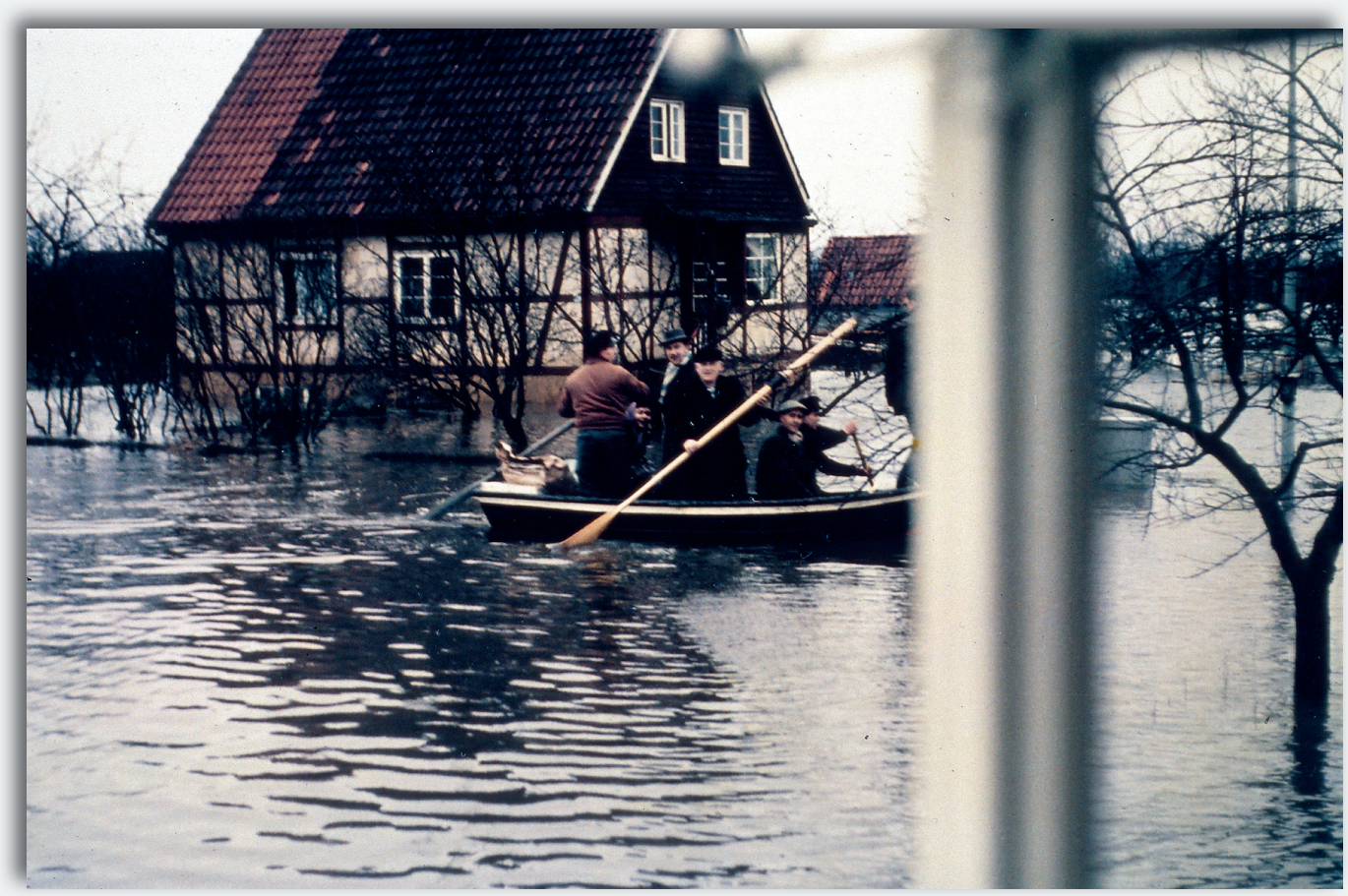

Frauke Paech

\title{
Die Hamburger Sturmflut von 1962
}

Eine Untersuchung aus erzählforschend-filmischer Perspektive 

Frauke Paech

Die Hamburger Sturmflut von 1962

Dieses Werk ist lizenziert unter einer

Creative Commons

Namensnennung - Weitergabe unter gleichen Bedingungen

4.0 International Lizenz.

(c) (1) ( ) 
erschienen als Band 12 in der Reihe „Göttinger Studien zur Kulturanthropologie/Europäischen Ethnologie“ im Universitätsverlag Göttingen 2020 
Frauke Paech

Die Hamburger Sturmflut von 1962

Eine Untersuchung aus

erzählforschend-filmischer Perspektive

Göttinger Studien zur

Kulturanthropologie/Europäischen

Ethnologie, Band 12

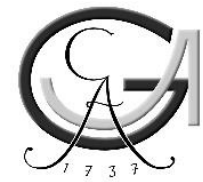

Universitätsverlag Göttingen

2020 


\section{Bibliographische Information der Deutschen Nationalbibliothek}

Die Deutsche Nationalbibliothek verzeichnet diese Publikation in der Deutschen Nationalbibliographie; detaillierte bibliographische Daten sind im Internet über $<$ http://dnb.dnb.de $>$ abrufbar.

„Göttinger Studien zur Kulturanthropologie/Europäischen Ethnologie“, herausgegeben von

Prof. Dr. Regina Bendix

E-Mail: rbendix@gwdg.de

Prof. Dr. Moritz Ege

E-Mail: mege@uni-goettingen.de

Prof. Dr. Sabine Hess

E-Mail: shess@uni-goettingen.de

Prof. Dr. Carola Lipp

E-Mail: Carola.Lipp@phil.uni-goettingen.de

Dr. Torsten Näser

E-Mail: tnaeser1@gwdg.de

Georg-August-Universität Göttingen

Institut für Kulturanthropologie/Europäische Ethnologie

Heinrich-Düker-Weg 14

37073 Göttingen

Dissertation, Universität Hamburg

Dieses Buch ist auch als freie Onlineversion über die Homepage des Verlags sowie über den Göttinger Universitätskatalog (GUK) bei der Niedersächsischen Staats- und Universitätsbibliothek Göttingen (https://www.sub.uni-goettingen.de) erreichbar. Es gelten die Lizenzbestimmungen der Onlineversion.

Satz und Layout: Sascha Bühler

Titelabbildung: Nachbarn rudern durch die Siedlung in Hamburg-Kirchdorf im Februar 1962. (Foto: Jürgen Ahrens)

(C) 2020 Universitätsverlag Göttingen

https://univerlag.uni-goettingen.de

ISBN: 978-3-86395-442-0

DOI: https://doi.org/10.17875/gup2020-1250

eISSN: 2512-7055 


\section{Inhalt}

Danksagung. . . . . . . . . . . . . . . . . . . .

Link zum Film Flut 1962 - Erinnern. Gedenken. Erzählen. . . . . . . . . .9

1 Einleitung: Mit Film über eine Katastrophenerfahrung erzählen $\ldots .11$

1.1 „Erinnern. Gedenken. Erzählen.“ - Forschungszugänge . . . . . . . . 16

1.2 Erfahrungen als gemeinschaftliche Erzählung . ............25

2 Erzählforschung über die Hamburger Sturmflut von 1962 mit Film -

Forschungsstand und -perspektiven . . . . . . . . . . . . . . . 29

2.1 Die Hamburger Sturmflut von 1962 als Forschungsfeld:

bisherige Zugänge und Untersuchungen................. 31

2.2 Der Begriff, Naturkatastrophe und der Mensch:

Zuschreibungsproblematiken .....................40

2.3 Katastrophenforschung: volkskundlich-kulturanthropologische

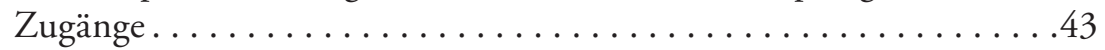

2.4 Katastrophenkultur zwischen Vulnerabiltät und Resilienz . . . . . . .47

2.5 Erzählforschung: Reden über katastrophische Erfahrungen . . . . . . . 52

2.6 Erzählen im Film . . . . . . . . . . . . . . . . . . . . . . . .57

2.6.1 Der ethnografische Film und Interviews . . . . . . . . . . 59

2.6.2 Eberhard Fechner. . . . . . . . . . . . . . . . . . .62

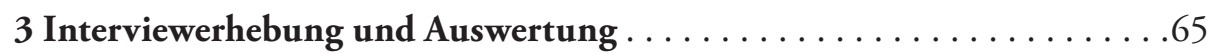

3.1 Interviews als kommunikative Praxis . . . . . . . . . . .66

3.1.1 Ermittlung von Interviewpartner_innen . . . . . . . . .67

3.1.2 Interviews erheben. . . . . . . . . . . . . . . . . . .69

3.1.3 Interviewtranskriptionen - ein Problemaufriss. . . . . . . . . . . 70

3.2 Interviews im Wissensformat Film . . . . . . . . . . . . . . . . 72

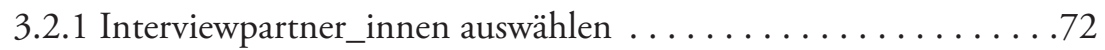

3.2 .2 Interviews filmen . . . . . . . . . . . . . . . . . 73

3.2 .3 Interviews auswerten $\ldots \ldots \ldots \ldots \ldots \ldots \ldots \ldots \ldots \ldots \ldots$

3.2 .4 Interviews montieren. . . . . . . . . . . . . . 76 
4 Der Film Flut 1962 - Erinnern. Gedenken. Erzäblen.. . . . . . . . . 79

4.1 Filmteil 1: Erzählen mit zeitgenössischen Fotografien..........85

4.1.1 Medienamateur_innen und ihre Fotomotive . . . . . . . . .85

4.1.2 Fotoalben als Erzählanlass . . . . . . . . . . . . . . . . .87

4.2 Filmteil 2: Erzählen als persönliche Masternarration ............92

4.2.1 Muster des Erzählens . . . . . . . . . . . . . . . . . .92

4.2.2 Leitmotive des Erzählens . . . . . . . . . . . . . . . . . . . .98

4.2.3 Leerstellen des Erzählens ... . . . . . . . . . . . . . . . . . 106

4.2.4 Zusammenfassung: Erzählen als Wiederherstellung von

Ordnung...........................

4.3 Filmteil 3: Erzählen im Kontext von Resilienz und Vulnerabilität . . .117

4.3.1 Erzählhaltungen zwischen Erfahrungswissen und

Risikobewusstsein .........................118

4.3.2 Angst als Argument . . . . . . . . . . . . . . . . . . . . 120

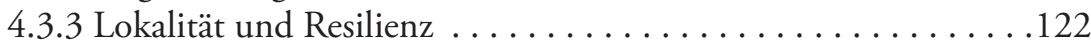

4.3.4 Cultures of Awareness ........................... . . . . . . . . .

5 Fazit: Erzählforschung und Film - Warum Worte Bilder brauchen . ..127

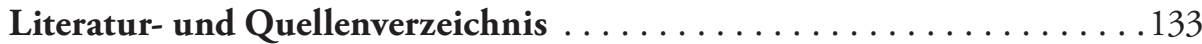

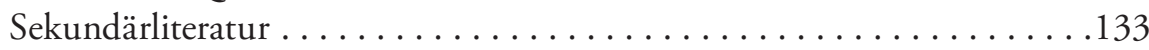

Gedruckte Quellen.................................149

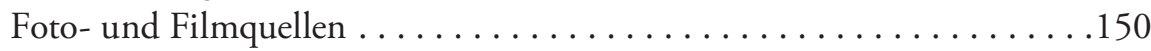

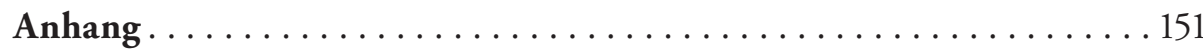

Interviewsample - Biografische Informationen..............151 


\section{Danksagung}

Diese Untersuchung wurde durch die Unterstützung vieler ermöglicht:

Mein größter Dank gilt meinen Interviewpartner_innen. Die Bereitschaft, mir ihre persönlichen und innerfamiliären Erfahrungen zu schildern, gewährte mir berührende und lehrreiche Einblicke in die Zeit während der Sturmflut von 1962 bis heute. Ihr Vertrauen und die Aufgeschlossenheit, sich auch vor der Kamera interviewen zu lassen, ermöglichten es mir, diese Untersuchung durchzuführen.

Ich danke meinen Gutachtern Albrecht Lehmann und Norbert Fischer am Institut für Volkskunde/Kulturanthropologie der Universität Hamburg, die die Entstehung und Entwicklung dieser Untersuchung stets anregend begleiteten. Darüber hinaus war ihre Offenheit gegenüber einem transmedialen Zugang von essenzieller Bedeutung. Zu Dank verpflichtet bin ich der Forschungsstelle für Zeitgeschichte in Hamburg, wo ich als wissenschaftliche Mitarbeiterin der ,Werkstatt der Erinnerung diese Untersuchung begonnen habe, insbesondere Dorothee Wierling für die notwendigen kritischen Hinweise sowie an Angelika Voß-Louis. Des Weiteren danke ich den Mitarbeiter_innen der Fotostelle der Universität Hamburg. Die finanzielle Unterstützung der Ida Lohmann-Siems Stiftung ermöglichte es mir, den Film Flut 1962 - Erinnern. Gedenken. Erzählen. fertigzustellen. Großen Dank schulde ich Andree Kummerfeld für seine ausdauernde und präzise Arbeit als Kameramann und als Cutter. Damit hat er wesentlichen Anteil an der filmtechnischen Produktion. Margret Markert, die Leiterin der Geschichtswerkstatt Wilhelmsburg \& Hafen, war 
meine erste Anlaufstelle im Feld und wurde im Laufe der Jahre von einer keyperson zu einer Freundin, danke. Maria Akingunsade konnte ich für die Interviewtranskriptionen gewinnen, eine langjährige Zusammenarbeit darüber hinaus verbindet auch uns. Für die umsichtige Satzlegung und Fertigstellung dieser Publikation danke ich Sascha Bühler sowie Petra Lepschy vom Universitätsverlag Göttingen.

Die Theologische Bibliothek der Universität Hamburg und das Archiv der deutschen Frauenbewegung in Kassel boten eine ideale Arbeitsatmosphäre für die Fertigstellung dieser Untersuchung. Mein Dank geht in diesem Zusammenhang an Hannelore Wilke sowie an die Mitarbeiterinnen des Archivs, auch für ihr zugewandtes Interesse an meiner Befindlichkeit. Bedanken möchte ich mich sehr für wertvolle Anregungen und für die zielführende Unterstützung, den friendly push, bei Regina Bendix und Torsten Näser am Institut für Kulturanthropologie/Europäische Ethnologie der Georg-August Universität Göttingen. Für die Begleitung dieser Arbeit, für die Unterstützung und für die Ermutigungen danke ich von Herzen zudem Christine Bischoff, Gerrit Herlyn, Inga Klein, Leonie Koch, Margarete Kranz, Janina Kriszio, Thomas Kuhn, Heike Perrakis, Janine Schemmer und Sonja Windmüller. Mein besonderer Dank gilt meiner Mutter, Margret Paech, und vor allem Barbara Günther, die mein Vorhaben immer unterstützt und unermüdlich begleitet hat. 
Link zum Film Flut 1962 - Erinnern. Gedenken. Erzählen.

(2007, 60 Minuten)

https://doi.org/10.5446/45883

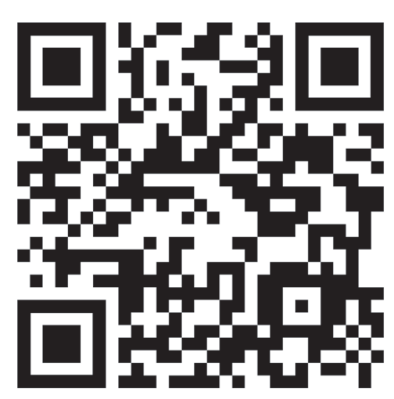





\section{Einleitung: Mit Film über eine Katastrophenerfahrung erzählen}

„Liebe Wilhelmsburgerinnen und Wilhelmsburger, wir haben uns heute hier wieder versammelt, wie jedes Jahr, um gemeinsam der Opfer jener Tage zu gedenken." Mit diesen Worten beginnt der Ortsamtsleiter Heiko Schulz, als behördlicher Vertreter der Stadt Hamburg, seine Rede an die Teilnehmer_innen einer Gedenkveranstaltung am Wilhelmsburger Flutdenkmal am Abend des 16. Februars 2004 aus Anlass der Hamburger Sturmflut von 1962. Anwesend sind neben Vertreter_innen der Wilhelmsburger Deichwacht und der Freiwilligen Feuerwehr hauptsächlich Zeitzeug_innen und deren Angehörige.

In der beschriebenen Szene sehen wir Menschen versammelt, die ich als Erinnerungsakteur_innen ansehe, denn sie repräsentieren eine Personengruppe, die Jahr um Jahr dieser Veranstaltung beiwohnt. Bei der Sturmflut von 1962 starben auf Hamburger Stadtgebiet über 315 Menschen, aber zigtausende Einwohner_innen haben dieses Ereignis überlebt, und viele von ihnen können auch heute noch davon erzählen. Somit verweist die beschriebene Szene auf das Forschungsinteresse dieser Untersuchung, nämlich, mit Albrecht Lehmann, dem Nestor einer kulturwissenschaftlichen Erzählforschung als Bewusstseinsanalyse ausgeführt, auf das „Reden 
über Erfahrung “. ${ }^{1}$ Diese Ansprache ist zugleich Bestandteil der ins Thema einführende Sequenz des von mir produzierten Dokumentarfilms Flut 1962 - Erinnern. Gedenken. Erzählen. ${ }^{2}$

Das erkenntnisleitende Vorgehen dieser Untersuchung besteht darin, die erhobenen Interviewergebnisse auf das audiovisuelle Medium Film zu übertragen. Wenn auch ein selten gewählter Weg für wissenschaftliche Qualifikationen, so sind Dokumentarfilme dennoch eine seit Jahrzehnten anerkannte wissenschaftliche Repräsentationsform. ${ }^{3}$ Für die Volkskunde/Kulturanthropologie und deren auf den Alltag und seine Verarbeitung fokussierten empirischen Forschungsmethoden bietet das Wissensformat Film die Möglichkeit, Ergebnisse niedrigschwellig, publikumswirksam und erfahrungsnah zu vermitteln, was gerade für diese Wissenschaft seit jeher ein wichtiges, wenn auch komplexes Anliegen darstellt. ${ }^{4}$

In meinen schriftlichen Ausführungen lehne ich mich an die Dissertationen von Lisa Röösli und Marius Risi an, die zu ihren je individuell erstellten Dokumentarfilmen eine gemeinsame Publikation mit „begleitenden Forschungsberichten“ veröffentlichten. ${ }^{5}$ Auch im Folgenden werden empirische und analytische Ergebnisse sowie wesentliche Aspekte, die die Produktion von Flut 1962 - Erinnern. Gedenken. Erzählen. bestimmten, ausgeführt. Oder mit Risi formuliert, greift auch dieser Text

„den Bildern dort mit Worten unter die Arme, wo sie hinsichtlich einer kulturanthropologischen Betrachtung besonders erklärungsbedürftig sind - an anderen Stellen überlässt er sie hingegen ganz bewusst der Seh- und Urteilskompetenz der Betrachterinnen und Betrachter. " ${ }^{\text {"6 }}$

Thematisch steht im Mittelpunkt dieser Untersuchung das Erzählen über die Hamburger Sturmflut von 1962. Dabei interpretiere ich das Erzählen als sozial und kulturell geformte Handlungspraxis und damit verbunden nicht nur als erinnerungskulturell relevantes Geschehen, sondern auch als mögliche Strategie der Erfahrungsbewältigung. Durch die Analyse selbst erhobener, qualitativer Interviews mit damals Betroffenen dieses Extremereignisses können induktiv differenziertere Interpretati-

1 Lehmann, Albrecht: Reden über Erfahrung. Kulturwissenschaftliche Bewusstseinsanalyse des Erzählens. Berlin 2007.

2 Der Film Flut 1962 - Erinnern. Gedenken. Erzählen. (2007, 60 Minuten) ist abrufbar unter https://doi.org/10.5446/45883.

3 Ausführlich hierzu siehe Ballhaus, Edmund (Hg.): Dokumentarfilm: Schulen - Projekte Konzepte. Berlin 2013 (abgekürzt: 2013a); Ruby, Jay: Picturing Culture. Explorations of Film \& Anthropology. Chicago 2000.

4 Vgl. Ballhaus, Edmund (Hg.): Kulturwissenschaft, Film und Öffentlichkeit. Münster 2001.

5 Vgl. Leimgruber, Walter: Ethnografischer Film: (un)geliebtes Stiefkind der Kulturwissenschaft. In: Röösli, Lisa/Risi, Marius: Lebensbilder - Bilderwandel. Zwei ethnografische Filmprojekte im Alpenraum. Münster 2010, S. 9-24, S. 9.

6 Risi, Marius: Im Lauf der Zeiten. Oberwalliser Lebenswelten. In: Röösli, Lisa/Ders.: Lebensbilder - Bilderwandel. Zwei ethnografische Filmprojekte im Alpenraum. Münster 2010S. 149-254, S. 152. 
onsweisen der in der Gesellschaft verankerten Haupterzählung freigelegt werden. Im Kontext von Katastrophenschutzmanagement und -prävention gelten Zeitzeug_innen hierzulande nach wie vor zumeist als wenig relevante Größe, anders als etwa in der US-amerikanischen Katastrophenforschung, wo deren Erfahrungswissen Anerkennung findet im Kontext von Untersuchungen zu Vulnerabilität (der Anfälligkeit einer Gesellschaft) und Resilienz (der gesellschaftlichen Widerstandsfähigkeit und dem Vermögen zur Regeneration), da diese Konzepte wesentlich als sozial determiniert interpretiert werden. ${ }^{7}$

Die erhobenen Erinnerungserzählungen werden im Rahmen dieser Untersuchung innerhalb ihres semantischen Gehalts und vor allem als subjektive Deutungspraktiken interpretiert: erstens im Kontext damaliger Handlungsfelder und zweitens - filmisch dargestellt - als Repräsentation und Performanz von Erfahrungswissen. Daran anknüpfend können drittens Rückschlüsse auf die heutige und womöglich zukünftige Relevanz dieser erinnerungskulturellen Praxis des Erzählens gezogen werden.

Das Erzählen damals betroffener Frauen und Männer steht somit im Mittelpunkt des Films Flut 1962 - Erinnern. Gedenken. Erzählen. Im Jahr 2004 führte ich insgesamt 53 Interviews, 31 wurden mit einer Videokamera aufgezeichnet. ${ }^{8}$ Anschließend erfolgte auf methodisch-analytischer Ebene die Zusammen- und Weiterführung praxeologischer Ansätze, konkret eine Verknüpfung von Erzählforschung und filmischer Repräsentation durch die Konzeptentwicklung und Produktion eines volkskundlich-kulturanthropologischen Dokumentarfilms. Mit dem Kulturwissenschaftler Edmund Ballhaus formuliert meint dies, dass diese filmische Arbeit geleitet ist von dem Anspruch „kulturwissenschaftliches Erkenntnisinteresse und fachspezifische Methoden wie eine Folie über alle Schritte der Filmproduktion zu legen“.? Dabei orientiere ich mich weniger an Traditionen des volkskundlichen Films, sondern ich nutze das Medium Film zur Repräsentation von Forschungsergebnissen bzw. als Wissensformat, wie oben bereits dargelegt wurde. ${ }^{10}$

In ihrem programmatischen Aufsatz zur Methodologie der Visuellen Anthropologie sprechen sich auch Walter Leimgruber, Silke Andris und Christine Bischoff dafür aus, diesen Forschungszugang nicht primär dafür zu nutzen, „bestehendes

Vgl. Oliver-Smith, Anthony/Hoffman, Susanna M.: Introduction. Why Anthropologists Should Study Disasters. In: Dies. (Hg.): Catastrophe \& Culture. The Anthropology of Disaster. Oxford 2002, S. 3-22, S. 3.

8 Detaillierte biografische Informationen zum Interviewsample siehe im Anhang.

9 Ballhaus, Edmund: Kulturwissenschaftliche Erkenntnis als dokumentarische Inszenierung. Dokumentarfilm in Göttingen. In: Ders. (Hg.): Dokumentarfilm: Schulen - Projekte - Konzepte. Berlin 2013, S. 234-264, S. 236 (abgekürzt: 2013b).

10 Eine programmatisch kritische Betrachtung des volkskundlichen Films siehe Ballhaus, Edmund: Film und Feldforschung. In: Ders./Engelbrecht, Beate (Hg.): Der ethnographische Film. Eine Einführung in Methoden und Praxis. Berlin 1995, S. 13-46 (abgekürzt: 1995a); Engelbrecht, Beate: Film als Methode in der Ethnologie. In: Ballhaus, Edmund/Dies. (Hg.): Der ethnographische Film. Eine Einführung in Methoden und Praxis. Berlin 1995, S. 143-186. 
anthropologisches Wissen zu bestätigen oder zu illustrieren, sondern als Mittel, neues Wissen zu generieren“. ${ }^{11}$ Die Autor_innen stellen in Anlehnung an den Ethnologen und Dokumentarfilmer David MacDougall zwei idealtypische filmische Formate im Forschungsprozess einander gegenüber, die auch die Arbeitsschritte für Flut 1962 - Erinnern. Gedenken. Erzählen. bestimmen. ${ }^{12}$ Zum einen verwendet MacDougall den Begriff „extension of the eye“. Im Zusammenwirken von Filmemacher_in, Kamerapraxis und gefilmten Protagonist_innen entsteht sogenanntes Rohmaterial, das zur eigenen Erkenntnisgenerierung im Forschungsprozess sowie ggf. für die anschließende Illustration einer schriftlichen Analyse produziert wird. ${ }^{13}$ Zum anderen, von MacDougall als „extension of the mind“ bezeichnet, steht das abgeschlossene filmische Werk, das einem narrativen Strang folgt und die deutliche Handschrift der Filmemacherin bzw. des Filmemachers trägt. Die Kamera fungiert hier „mit dem Ziel, thematische und formale Konzepte zu verwirklichen “14 sowie in Hinblick darauf, das Endprodukt Film einer Öffentlichkeit zugänglich zu machen und somit gegebenenfalls auch auf das Feld zurückzuwirken.

Die im Rahmen dieser Forschungsarbeit praktizierte filmische Vorgehensweise umfasst zunächst die Datenerhebung, also die Erhebung von Rohmaterial in Form der gefilmten Interviews. Nach ihrer Auswertung erfolgt die Transformation dieses Materials in ein narrativ gestaltetes filmisches Endprodukt, in ein Wissensformat, in dem die zentralen Untersuchungsergebnisse dargestellt werden. Somit fungiert Flut 1962 - Erinnern. Gedenken. Erzählen. als extension of eye and mind. Quasi ,dazwischengeschaltet' sind erkenntnisgenerierende Prozesse der Interviewanalyse und -interpretation sowie die Produktion weiterer, darstellungsrelevanter Filmaufnahmen, des Weiteren die Reflexionsprozessen unterworfene Einbeziehung von zeitgenössischem Foto- und Filmmaterial sowie insgesamt die Montage als methodisches Instrument mit „selektierenden, narrativierenden und rhythmisierenden Eigenschaften". 15

Dieses mit großem Arbeits- und Zeitaufwand durchgeführte Vorgehen unterliegt nicht nur analytischen Prämissen, sondern zusätzlich auch filmisch-narrativen Gestaltungs- und Darstellungskonventionen, damit die filmische Gesamtaussage innerhalb

${ }_{11}$ Leimgruber, Walter/Andris, Silke/Bischoff, Christine: Visuelle Anthropologie: Bilder machen, analysieren, deuten und präsentieren. In: Hess, Sabine/Moser, Johannes/Schwertl, Maria (Hg.): Europäisch-ethnologisches Forschen. Neue Methoden und Konzepte. Berlin 2013, S. 247-281, S. 276, mit Bezug auf MacDougall, David: Visual Anthropology and the Ways of Knowing. In: Ders.: Transcultural Cinema. Princeton 1998, S. 61-92.

12 Vgl. MacDougall, David: The Visual in Anthropology. In: Banks, Marcus/Morphy, Howard (Hg.): Rethinking Visual Anthropology. New Haven 1997, S. 276-295, S. 292.

13 Eine kritische Würdigung dieser Vorgehensweise siehe Hänel, Dagmar: Anmerkungen zum gefilmten Interview in der Volkskunde. In: Wossidlo, Joachim/Roters, Ulrich (Hg.): Interview und Film. Volkskundliche und Ethnologische Ansätze zu Methodik und Analyse. Münster u. a. 2003, S. 107-118.

14 Leimgruber/Andris/Bischoff 2013, S. 263.

15 Näser, Torsten: Film und Text. Ethnografische Wissensformate im Diskursvergleich. Berlin 2014, S. 249. 
der jeweiligen Veröffentlichungsarenen ${ }^{16}$ sowohl für Fachwissenschaftler_innen als auch für ein insgesamt heterogenes Publikum plausibel und nachvollziehbar wird. ${ }^{17}$

Seit 2007 wurde Flut 1962 - Erinnern. Gedenken. Erzählen. wiederholt gezeigt, zuletzt 2012 im Rahmen von mehreren Veranstaltungsreihen zum 50. Gedenkjahr an die Sturmflut von 1962. Aufgrund seiner mittlerweile mehreren hundert Zuschauer_innen wird er womöglich mittlerweile als ein Bestandteil dessen rezipiert, worüber dieser Film erzählt, nämlich als eine weitere erinnerungskulturelle Repräsentation der damaligen Ereignisse.

Der Film thematisiert im ersten Teil die Bedeutung von zeitgenössischen Fotografien und Fotoalben als Bestandteil innerfamiliärer Tradierungspraktiken von Erinnerungserzählungen. ${ }^{18}$ Durch ihren zugleich medialen Repräsentationsgehalt stellen diese Objekte einen wesentlichen Referenzrahmen für den Austausch und für die Vermittlung von Erfahrungen dar. Der zweite Teil des Films widmet sich konkret den Erinnerungserzählungen über die Ereignisse vom 16. und 17. Februar 1962. Interviewauszüge aus den Haupterzählungen bilden hier den ausführlichsten und mit 40 Minuten Dauer auch den längsten Teil des Films. Der dritte Teil thematisiert heutige Haltungen und Strategien der Bewältigung dieser Extremerfahrung. Er bildet das filmische Fazit und generiert gleichzeitig einen „nachvollziehbaren Ausstieg, eine[n] logischen Schluss “. ${ }^{19}$ Dieser dritte Filmteil hat wie der erste eine Länge von ca. 10 Minuten, beide Teile fungieren als argumentative und dramaturgische Rahmung des mittleren Teils. Als filmische Leitmotive nutze ich neben Filmmusik zudem selbst gedrehte Filmaufnahmen von Orten des damaligen Geschehens sowie von heutigen Artefakten einer potenziellen Hochwasserbedrohung (z. B. Hinweisschilder und Hochwasserschutzanlagen), des Weiteren Aufnahmen von Gedenksymbolen und -orten, wie etwa von der Neuenfelder St. Pankratiuskirche, die der

16 Der Begriff „Veröffentlichungsarena“ bezeichnet den Aufführungskontext eines filmischen Formats. Vgl. Eckardt, Sandra/Näser, Torsten: Ethnografisches Filmen. In: Bischoff, Christine/ Oehme-Jüngling, Karoline/Leimgruber, Walter (Hg.): Methoden der Kulturanthropologie. Bern 2014, S. 273-290, S. 273.

17 Manfred Hattendorf spricht in diesem Zusammenhang von der Erfüllung eines Wahrnehmungsvertrags zwischen Publikum und Filmemacher_in. Vgl. Hattendorf, Manfred: Dokumentarfilm und Authentizität. Ästhetik und Pragmatik einer Gattung. Konstanz 1994, S. 71. Weiterführend siehe Engelbrecht, Beate: For Whom Do We Produce? In: Crawford, Peter Ian (Hg.): The Construction of the Viewer. Aarhus 1996, S. 163-176.

18 Innerhalb einer Volkskunde/Kulturanthropologie, die ,Kultur' in einem auch „ikonologischen Kontext" verortet, ergeben sich mit Ulrich Hägele breit gefächerte Forschungszugänge: „[s] owohl die Geschichte der Bilder als Artefakte der Erinnerung, als auch Sehgewohnheiten, Codierungen, mediale Wahrnehmungsprozesse und Authentizität. Es geht um die Zusammenhänge von Bild, Kontext und Bedeutung und um deren Wandel im digitalen Zeitalter." Hägele, Ulrich: Visuelle Kultur? Thesen zum erweiterten Fachverständnis bildmedialer Forschung. In: Gerndt, Helge/Haibl, Michaela (Hg.): Der Bilderalltag. Perspektiven einer volkskundlichen Bildwissenschaft. Münster u. a. 2005, S. 375-388, S. 384. 
Bevölkerung damals als Fluchtort diente. Mit diesen Aufnahmen ist sowohl intendiert, auf einer konnotativen Ebene zu antizipieren, was die Befragten vor ihrem inneren Auge sehen, als auch auf einer denotativen Ebene auf anhaltende bzw. zukünftige Gefahrenlagen auf Hamburger Stadtgebiet hinzuweisen. Um diesen Aussageabsichten eine weitere Argumentationsebene hinzuzufügen, finden im ersten und zweiten Filmteil Film- und Fotoaufnahmen von den damals überfluteten Gebieten Verwendung. Der Titelzusatz des Films „Erinnern. Gedenken. Erzählen.“ verweist somit auch auf die Interdependenzen dieser zeithistorischen Quellen mit heutigen selbstproduzierten Filmaufnahmen. Im Folgenden werden die Termini näher bestimmt und ihre Relevanz für diese Untersuchung begründet.

\section{1 „Erinnern. Gedenken. Erzählen.“ - Forschungszugänge}

Autobiografisches Erinnern hat grundsätzlich, also in seinem alltäglichen Gebrauch, mehrere Funktionen. Der Psychologe Rüdiger Pohl skizziert diese Nutzungsweisen folgendermaßen: Erstens lassen sich selbstbezogene Funktionen des autobiografischen Erinnerns konstatieren, also Motive, die auf „die Bildung und Akzentualisierung eines Selbstkonzepts“ abzielen. Individuelle Erfahrungen werden dabei als „eine kohärente Lebensgeschichte (life story) “ verstanden, „die uns das Gefühl von Identität (im Sinne persönlicher Konsistenz) und Wachstum vermittelt “. ${ }^{20}$ In Verbindung dazu stehen zweitens sogenannte sozial-kommunikative Funktionen des autobiografischen Erinnerns, diese zielen auf „die Herstellung und Aufrechterhaltung sozialer Bezüge, wie auch Akte der Selbstoffenbarung oder das Zeigen von Empathie“ ab. Im verbalen Austausch, so Pohl, also „dem miteinander Teilen persönlicher Erlebnisse (memory sharing), geben wir etwas von uns preis und hoffen auf entsprechende Reaktionen." ${ }^{21}$ Drittens lassen sich direktive Funktionen des autobiografischen Erinnerns identifizieren, also, laut Pohl, „die Bereitstellung von Wissen, Einstellungen und Meinungen, die Fähigkeit zur Enkulturation, die Hilfe beim Problemlösen und bei der Planung zukünftiger Handlungen sowie die Erfahrungsweitergabe an andere. "22

Dies mag als eine tendenziell zu konsistente Darstellung funktionaler Bereiche des autobiografischen Gedächtnisses anmuten, denn eine dark side, etwa Tabus und Brüche, scheinen dieser Konzeptualisierung, also dessen, was durch Erinnerungsleistungen im Gedächtnis behalten und verarbeitet oder auch vergessen wird, nicht inhärent. Für die hier untersuchten Erinnerungserzählungen ist diese Schematik jedoch gewinnbringend, weil sie zum einen die grundsätzliche Gerichtetheit auto-

20 Pohl, Rüdiger: Was ist Gedächtnis/Erinnerung? Das autobiographische Gedächtnis. In: Gudehus, Christian/Eichenberg, Ariane/Welzer, Harald (Hg.): Gedächtnis und Erinnerung. Ein interdisziplinäres Handbuch. Stuttgart/Weimar 2010, S. S. 75-84, S. 80. Hervorhebung im Original, mit Bezug auf Bluck, Susan: Autobiographical Memory. Exploring its Functions in Everyday Life. In: Memory 11 (2), 2003, S. 113-124.

21 Ebd. Hervorhebung im Original.

22 Ebd., S. 80f. 
biografischen Erinnerns auf die drei Zeitebenen Vergangenheit, Gegenwart und Zukunft expliziert, zum anderen, da sie zugleich drei relevante Untersuchungsfelder umreißt: erstens die „life story“ als genuinen Untersuchungsgegenstand einer Erzählforschung als Bewusstseinsanalyse, zweitens ein Film als Repräsentationsmedium inhärentes und nach außen adressiertes „memory sharing“ sowie drittens die Intentionalität selbst, also Vermittlungsabsichten, die auf durch erfahrungsgeschichtliches Lernen erworbenen Kompetenzen fußen. Diese drei subjektorientierten Funktionen autobiografischen Erinnerns sind für diese Untersuchung erkenntnisleitend, weil, anders als etwa bei der institutionalisierten Erinnerungskultur Hamburgs im Kontext von Holocaust, Luftkrieg und dem Ende des Zweiten Weltkriegs, wo öffentliches Gedenken einen „Erinnerungsrahmen für private und familiäre Erinnerungen “23 setzt, es in Bezug auf die Sturmflut von 1962 nur wenig ausgeprägt ist: Denkmäler und Gedenktafeln, Publikationen mit Erfahrungsberichten, Ausstellungen und Gedenkveranstaltungen sind zumeist auf lokale Initiativen zurückzuführen oder zumindest nicht ohne deren Engagement entstanden. ${ }^{24}$ Nördlich der Elbe, also dem geografisch und in Bezug auf die Bevölkerungszahlen größeren Teil des Stadtgebiets, befinden sich - mit Ausnahme weniger Flutmarken am Elbwanderweg, finanziert von der Innenbehörde ${ }^{25}$ sowie der Grabanlage auf dem im Norden Hamburgs gelegenen Ohlsdorfer Friedhof - keine von städtischer Seite initiierten Erinnerungsstätten.

Ein Grund hierfür mag das Dilemma sein, in dem sich die verantwortlichen Behördenvertreter_innen befinden. Es besteht darin, sowohl die Hochwassersicherheit Hamburgs proklamieren und technokratische Kompetenz beweisen zu wollen, als auch die umfassenden Katastrophennotfallprogramme bekannt zu machen und somit ein Risikobewusstsein der Bevölkerung wach zu halten. ${ }^{26}$ Dieser Vorbehalt in punkto Sicherheit scheint von Seiten städtischer Verantwortlicher zugunsten einer reduzierten Erinnerungskultur entschieden worden zu sein. Lediglich zu ,runden“ Gedenktagen und in Wahlkampfzeiten finden sich prominente Politiker_innen zu den Gedenkveranstaltungen ein. ${ }^{27}$ Gleichwohl scheint dieses Phänomen verallge-

23 Thießen, Malte: Drei Geschichten des „Feuersturms“. Erinnerungen von Zeitzeugen zwischen privaten, familiären und öffentlichen Erzählungen des Luftkriegs. In: Dierken, Jörg (Hg.): Geisteswissenschaften in der Offensive. Hamburger Standortbestimmungen. Hamburg 2009, S. 353-374, S. 368.

24 Dies gilt etwa für die Gedenkveranstaltungen anlässlich des 50. Jahrestages. Ausführlich hierzu siehe Mauch, Felix: Erinnerungsfluten. Das Sturmfluthochwasser von 1962 im Gedächtnis der Stadt Hamburg. München/Hamburg 2015, S. 245-252, mit Bezug u. a. auf Markert, Margret/ Paech, Frauke: 50 Jahre Flut. Projektbericht über die Veranstaltungsreihe zum 50sten Gedenkjahr an die Hamburger Sturmflut von 1962 in Wilhelmsburg. Geschichtswerkstatt Wilhelmsburg \& Hafen. Hamburg 2012.

25 Vgl. ebd., S. 172.

26 Siehe hierzu auch Fellmer, Mareike: Bürgerschaftliches Engagement und Sturmfluten. Ausprägungen und Einflussfaktoren am Beispiel des Flussgebietes der Tideelbe. Detmold 2014.

27 Vgl. Paech, Frauke: „Die ganzen menschlichen Geschichten“ - Die Hamburger Sturmflut von 1962 im Bewusstsein der Wilhelmsburger Bevölkerung. In: Geschichtswerkstatt Wilhelmsburg \& 
meinerbar zu sein. Der Historiker Christof Mauch stellt für das massenmediale Interesse in der heutigen, globalisierten Welt heraus:

„Memory of natural disasters is, in contrast to memory of war, markedly short-lived. As waters return to their preflood levels and the last victims of earthquakes are recovered, so, too, does mass-media interest in natural disasters quickly subside. ${ }^{28}$

Bereits 1981 gelangte der Historiker Arno Borst zu der Auffassung, dass europäische Gesellschaften einen großen Vorbehalt gegenüber katastrophalen Erfahrungen hätten, da es dem modernen „Selbstgefühl zutiefst“ widerstrebe, diese „als dauerhafte Erfahrung der Gesellschaft und der Geschichte anzunehmen“. Vielmehr würden die Ereignisse in eine Gegenwart „isoliert“ und aus der Vergangenheit „elimiert [...], weil sie die Zukunft nicht definieren sollen." ${ }^{29}$

Dieser Mangel an öffentlicher Aufmerksamkeit korrespondiert nicht mit dem Stellenwert, den die Hamburger Sturmflut von 1962 für die damals betroffenen Bevölkerungsteile hat. Auch die bereits erwähnten, im Jahr 2012 primär von lokalen Erinnerungsakteur_innen, wie der Geschichtswerkstatt Wilhelmsburg \& Hafen, Kirchengemeinden und Heimatvereinen initiierten, organisierten und durchgeführten Veranstaltungsreihen zum 50. Jahrestag können daher sowohl als Ausdruck eines offensiven Umgangs mit der eigenen Geschichte als auch als Bedürfnis interpretiert werden, die damaligen Erlebnisse in der Öffentlichkeit ,wach ' zu halten. Hier ließe sich demgemäß von einem bürgerschaftlich organisierten Erinnerungsrahmen sprechen, der wiederum das öffentliche Gedenken rahmt. ${ }^{30}$

Vor diesem Hintergrund verweist der Titelzusatz des Films „Erinnern. Gedenken. Erzählen." auf drei komplexe Ebenen der Tradierung und Vergegenwärtigung sowie auf eine Perspektivierung in die Zukunft, die miteinander in enger Beziehung stehen.

Hafen/Museum Elbinsel Wilhelmsburg (Hg.): Wilhelmsburg. Hamburgs große Elbinsel.

Hamburg 2008, S. 161-173, S. 162.

28 Mauch, Christof: Introduction. In: Ders./Pfister, Christian (Hg.): Natural Disasters, Cultural

Responses. Case Studies towards a Global Environmental History. Lanham u. a. 2009, S. 1-16,

S. 3.

29 Vgl. Borst, Arno: Das Erdbeben von 1348. Ein historischer Beitrag zur Katastrophenforschung. In: Historische Zeitschrift 233 (3), 1981, S. 529-569, S. 532. Und auch Frank Uekötter kommt 2014 zu einem ähnlichen Ergebnis, wenn er im Kontext von „Erinnerungsorten“ (Pierre Nora) das Fehlen von „Naturkatastrophen“ dahingehend interpretiert, dass es sich hierbei „letztlich um störrische Ereignisse“ handele, „die wie erratische Blöcke in das Gefüge der menschlichen Geschichte einfallen. “ Uekötter, Frank: Wege zu einer ökologischen Erinnerungskultur. In: Ders. (Hg.): Ökologische Erinnerungsorte. Göttingen 2014, S. 7-26, S. 18.

30 Trotz einer, wie oben angeführt, ambivalenten Haltung wurde die größte der Veranstaltungsreihen 2012 finanziell ermöglicht durch die Hamburger Behörde für Stadtentwicklung und Umwelt (BSU) und die Senatskanzlei der Freien und Hansestadt Hamburg. Zu dieser Veranstaltungsreihe siehe Markert/Paech 2012. 
Zunächst sei erneut hervorgehoben, dass sich dieses Extremereignis dadurch ausweist, von vielen Menschen memoriert zu werden. Etwa 100.000 Menschen haben 1962 in den Hamburger Stadtteilen gelebt, die in der Nacht vom 16. auf den 17. Februar von Wassermassen überflutet wurden. Von erinnerungskultureller Relevanz ist dabei erstens, dass dies als überraschend und erstmalig auftretend rezipiert wurde, denn eine in ihren Ausmaßen vergleichbare Sturmflut hatte es in Hamburg zuletzt 1825 gegeben. ${ }^{31}$ Zweitens ist ein unbestreitbarer Folgenreichtum der Geschehnisse zu konstatieren: über 300 Tote, zehntausende obdachlose Menschen, zerstörte Infrastruktur und ein immens hoher finanzieller Schaden. ${ }^{32}$ Die Erinnerungen sind drittens verknüpft mit einer starken emotionalen Aufladung, die zu dem Bedürfnis führt, sich mit anderen darüber verbal auszutauschen, die Geschehnisse somit häufig gedanklich abzurufen. Dies ermögliche, hier erneut mit Pohl argumentiert, „so in gewisser Weise ein Wieder-Erleben (re-experiencing)“ der Erfahrungen. ${ }^{33}$ In diesem Zusammenhang hebt er eine primär bildhafte Vorstellung hervor, die sich in episodisch gestalteten Narrationen widerspiegele. Als hilfreiche und somit gängige Praxis im Erzählen gelte zudem die chronologische Organisation dieser Geschichten. ${ }^{34}$

Diese in Bezug auf das autobiografische Gedächtnis formulierten Kategorisierungen verweisen auf den hier zentralen Untersuchungsgegenstand, nämlich die Erinnerungserzählungen, und sie begründen eine Argumentation aus der Perspektive einer kulturwissenschaftlichen Erzählforschung als Bewusstseinsanalyse.

\section{Das Erzählen}

Sowohl aufgrund der seit 1962 publizierten Erfahrungsberichte ${ }^{35}$ als auch aufgrund der Ergebnisse dieser, auf Interviewerhebungen basierenden, Untersuchung ist davon auszugehen, dass die Erinnerungserzählungen an die Hamburger Sturmflut von 1962 nicht tabuisiert sind, sondern seit damals bei Gesprächsanlässen, etwa im innerfamiliären Rahmen, ausgetauscht werden. In den Erinnerungen sind somit nicht die Ereignisse per se memoriert, sondern die erzählten Geschichten. ${ }^{36}$ Dabei entspricht das Erzählen dem Grundbedürfnis nach persönlicher Verarbeitung und Entlastung, gepaart mit der Intention, sich die eigenen Erfahrungen begreifbar zu machen. Erklärungen für belastende Erlebnisse zu formulieren und eine das Geschehen ordnende Struktur führen in der Regel zu chronologisch ausgerichteten

31 Vgl. Fischer, Norbert: Einführung. In: Fiedler, Gudrun (Hg. im Auftrag des Stader Geschichtsund Heimatvereins): Stader Jahrbuch 2011. 50 Jahre Sturmflut 1962. Ereignis - Geschichte Gedenken. Stade 2011, S. 13-15 (abgekürzt: 2011a).

$32 \mathrm{Zu}$ den Ereignissen der Sturmflut von 1962, interpretiert als eine in der Gesellschaft verankerte Meistererzählung siehe ausführlich Kapitel 2.1.

33 Vgl. Pohl 2010, S. 75. Hervorhebung im Original.

34 Vgl. ebd., S. 76.

35 Ausführlich zur Bedeutung sogenannter, grauer Literatur`siehe Kapitel 2.1.

36 Vgl. Assmann, Aleida: Erinnerungsräume. Formen und Wandlungen des kulturellen Gedächtnisses. München 1999, S. 250. 
Narrationen, wie oben schon angedeutet wurde. Mit Regina Bendix gehe ich von der Prämisse aus, dass

„die erste, vielleicht grundlegendste Katastrophenhilfe, die sich Menschen leisten können, das Erzählen [ist]: es ordnet die Erfahrung, und setzt gleichzeitig den Bewältigungsprozeß in Gang, der schlußendlich dazu beitragen wird, die Katastrophe in die Geschichte einzureihen. "37

Wir haben es hierbei mit biografisch einschneidenden Erfahrungen zu tun, unabhängig davon, ob diese als traumatisierend bezeichnet werden können bzw. als solche erfahren wurden.$^{38}$ Dies ist aus volkskundlich-kulturanthropologischer Perspektive auch nicht ermittelbar. ${ }^{39}$ Von fachlichem Interesse ist vielmehr, dass Erzählpraktiken sachliche Zusammenhänge evozieren, in die die Erlebnisse als Erfahrungen eingeordnet sind. ${ }^{40}$

Das Erzählen als wichtigen Bestandteil von Erinnerungskultur auszuweisen, ist nicht nur von fachlicher Relevanz, sondern mit ihr geht eine gesellschaftliche einher, denn ein Ereignis ,in die Geschichte einzureihen“, ist eine Handlung der Verständigung auf überindividueller Ebene. ${ }^{41}$ Die Relevanz des Forschungsthemas ergibt sich somit nicht nur aufgrund aktueller gesellschaftlicher Diskurse zum Phänomen des Klimawandels, innerhalb dessen Erforschung sich eine Untersuchung auch der Hamburger Sturmflut von 1962 im Kontext einer Folgenforschung einordnen ließe, sondern auch daraus, dass das Ereignis nach wie vor in kollektiven Gedächtnissen präsent und somit, in die Geschichte eingereiht' ist. Dieses nährt sich wiederum aus Erzähl- und auch Bildtraditionen, die gesellschaftlich anerkannt sind und verstanden werden. ${ }^{42}$

Der Historiker Christian Pfister merkt hierzu an: „And yet memory is crucial for the development of coping strategies. " ${ }^{43}$ Angesprochen wird von ihm damit im Kontext von Erinnerungskultur ein mögliches, Lernen aus der Katastrophe'. Als Schlüsselbegriffe zur Erforschung des gesellschaftlichen Umgangs mit Naturgefahren

37 Bendix, Regina: Zwischen Chaos und Kultur. Zur Ethnographie des Erzählens im ausgehenden 20. Jahrhundert. In: Zeitschrift für Volkskunde 92 (1), 1996, S. 169-184, S. 170.

38 Vgl. Rieken, Bernd: Nordsee ist Mordsee. Sturmfluten und ihre Bedeutung für die Mentalitätsgeschichte der Friesen. Münster 2005, S. 302.

39 Aleida Assmann führt aus: „In kulturwissenschaftlicher Perspektive geht es beim Trauma um eine Gratwanderung des Erinnerns, die sich zwischen einer auf das Unheil fixierten Erstarrung einerseits und einer das Unheil reflexiv gänzlich überwindenden Bewältigung andererseits bewegt." Assmann, Aleida: Gedächtnis als Leitbegriff der Kulturwissenschaften. In: Musner, Lutz/Wunberg, Gotthart (Hg.): Kulturwissenschaften. Forschung - Praxis - Positionen. Freiburg i. Br. 2003, S. 27-47, S. 40.

40 Siehe hierzu Rieken, Bernd (Hg.): Erzählen über Katastrophen. Beiträge aus deutscher Philologie, Erzählforschung und Psychotherapiewissenschaft. Münster/New York 2016.

$41 \quad$ Vgl. Bendix 1996, S. 170.

42 Ausführlich hierzu siehe Kapitel 2.1.

43 Pfister, Christian: „The Monster Swallows You“. Disaster Memory and Risk Culture in Western Europe, 1500-2000. In: RCC Perspectives (1) 2011, S. 1-23, S. 3. 
sowie der Katastrophenvermeidung gelten Konzepte von Vulnerabilität und Resilienz. Studien fokussieren hier auf lokale, ebenso ländliche wie städtische, Milieus. ${ }^{44}$ Das Potenzial, das dabei Erzählungen von Betroffenen innewohnt, hat bisher kaum gesellschaftliche Beachtung gefunden. An diesem Punkt kann eine Erzählforschung als Bewusstseinsanalyse ansetzen, die Interviewpartner_innen und ihre repetitiven Praktiken des Erinnerns, Gedenkens und Erzählens als Protagonist_innen und somit als erinnerungskulturelle Akteur_innen begreift.

\section{Das Gedenken}

Den Terminus Gedenken interpretiere ich weniger als institutionalisierte Zusammenkunft, also als eine kollektive Ausdrucksform des historischen Gedächtnisses ${ }^{45}$ der Stadt Hamburg, sondern erzählend vermittelt in seiner Funktion als „eine Brücke zwischen Gegenwart und Vergangenheit, zwischen Individuum und Familie, Privatem und Öffentlichem". ${ }^{46}$ Es ist eine zwar gemeinschaftlich initiierte und an eine Öffentlichkeit gerichtete, aber individuell motivierte und grundierte Praxis. Gedenken kann, wie der Historiker Malte Thießen hervorhebt, somit als eine „kollektive und gegenwartsbezogene Erzählung der Vergangenheit" angesehen werden. ${ }^{47}$

Für einen in Gedenkpraktiken implizit eingeschriebenen „soziale[n] Verfestigungsprozeß von Vergangenheit“, hebt auch der Soziologe Harald Welzer die zeitliche Ebene der Gegenwart hervor. ${ }^{48}$ Vergangenheit zu begehen sei stets absichtsvoll, somit seien Gedenkpraktiken ein auch zeitlich ausgerichtetes Bewusstsein inhärent. Dieses zielt nicht nur auf Werte- und Kohärenzerhalt einer Gruppe ab, wie Welzer herausstellt. ${ }^{49}$ Darüber hinaus impliziert insbesondere die Performativität individueller, gemeinschaftlich gerahmter Erzählungen, wie sie auch in Flut 1962 - Erinnern. Gedenken. Erzählen. gezeigt werden, eine ebenso auf zukünftiges Handeln ausgerichtete gesellschaftliche Relevanz. Durch sie wird im Gedenken

44 Siehe hierzu Schott, Dieter: Naturkatastrophen und städtische Resilienz. Die Hamburger Sturmflut im Kontext städtischer Naturkatastrophen der Neuzeit. In: Heßler, Martina/Kehrt, Christian (Hg.): Die Hamburger Sturmflut von 1962. Risikobewusstsein und Katastrophenschutz aus zeit-, technik- und umweltgeschichtlicher Perspektive. Göttingen/Bristol 2014, S. 37-57; siehe hierzu Bankoff, Greg: Cultures of Disaster. Society and natural hazards in the Philippines. London 2003.

45 „[D]ie kollektiven Gedächtnisse ebenso wie die Gruppen, mit denen sie verbunden sind, existieren stets im Plural, während das historische Gedächtnis, das einen integrierenden Rahmen für viele Geschichten konstruiert, im Singular existiert“. Assmann 1999, S. 131. Assmann bezieht sich auf die Unterscheidung von Maurice Halbwachs zwischen kollektivem Gedächtnis und dem Gedächtnisbegriff in der Geschichtswissenschaft. Vgl. Halbwachs, Maurice: Das kollektive Gedächtnis. Frankfurt a. M. 1985 (1939), S. 72 f (abgekürzt: 1985b).

46 Vgl. Thießen 2009, S. 372.

47 Vgl. Thießen, Malte: Eingebrannt ins Gedächtnis. Hamburgs Gedenken an Luftkrieg und Kriegsende 1943 bis 2005. Hamburg 2007, S. 13.

48 Vgl. Welzer, Harald: Das kommunikative Gedächtnis. Eine Theorie der Erinnerung. 2. Auflage. München 2008, S. 233.

49 Vgl. ebd. 
das historische Bewusstsein offenbar. Der Historiker Jörn Rüsen spricht in diesem Zusammenhang von einer „Erinnerungsleistung“ mit „Gegenwarts- und Zukunftsaspekt", in der die Erfahrung zu "Orientierungen der Lebenspraxis verarbeitet" werde. $^{50}$ Ich untersuche demgemäß, ob die interviewten Frauen und Männer über ein Geschichtsbewusstsein verfügen, interpretiert im „Zusammenhang von Vergangenheitsdeutung, Gegenwartsverständnis und Zukunftsperspektive “. ${ }^{51}$ Daraus wäre zu folgern, dass auch der hier erhobenen Äußerungsform des Gedenkens und Erinnerns, nämlich dem Erzählen, eine auf die drei Zeitebenen bezogene, gesellschaftlich relevante Funktion innewohnt.

\section{Das Erinnern}

Der Terminus Erinnern beschreibt zunächst eine individualisierende Perspektivierung. Das, was erinnert wurde, zu äußern, geschieht allerdings zumeist in dem Bewusstsein, bereits einer Erzählgemeinschaft anzugehören. ${ }^{52}$ Somit werden sich die persönlichen Geschichten in bereits bewährte Erzählmuster dieser Gemeinschaft und in die Palette inhaltlicher Übereinkünfte einpassen, wie ich anhand des Begriffes persönliche Masternarration in Kapitel 2.1 ausführen werde. Für eine Auswertung solcherart letztendlich überindividuell gestalteter und adressierter Geschichten fällt es gleichwohl schwer, die persönliche, „die biografische Dimension der Erinnerung" zu ermitteln. In den Fokus des Erkenntnisinteresses rückt daher mit Thießen die analytisch konkreter zu fassende „biografische Dimension der Motive der Erinnerung. " ${ }^{53}$ Auch diese Motive sind nicht ausschließlich bezogen auf eine erzählerische Gegenwart, sondern ebenfalls grundiert durch vergangene Erfahrungen und sie können absichtsvoll sein, etwa wenn sie Handlungsorientierungen für die Zukunft implizieren.

Diese pluralen Perspektiven einer Analyse von Erinnerungserzählungen gelten insbesondere dann, wenn es sich (wie bei den hier erhobenen) um Geschichten handelt, die ein ,gutes Ende' nehmen. ${ }^{54}$ Die biografische Dimension eines Erinne-

50 Vgl. Rüsen, Jörn: Historische Orientierung. Über die Arbeit des Geschichtsbewußtseins, sich in der Zeit zurechtzufinden. Köln/Weimar/Wien 1994, S. $6 f$.

51 Vgl. Jeismann, Karl-Ernst: Geschichtsbewußtsein. In: Bergmann, Klaus u. a. (Hg.): Handbuch der Geschichtsdidaktik. 4. Auflage. Seelze 1992, S. 40-43, S. 40.

52 Vgl. Nünning, Ansgar: Wie Erzählungen Kulturen erzeugen. Prämissen, Konzepte und Perspektiven für eine kulturwissenschaftliche Narratologie. In: Strohmaier, Alexandra (Hg.): Kultur - Wissen - Narration. Perspektiven transdisziplinärer Erzählforschung für die Kulturwissenschaften. Bielefeld 2013, S. 15-53, S. 44f. Nünning folgert, es sei „heuristisch produktiv, Kulturen als Erzählgemeinschaften zu konzeptualisieren, denn es sind ganz wesentlich die kollektiv geteilten Geschichten, die zur sozialen Kohärenzbildung, zur Entstehung von Deutungs- und Interpretationsgemeinschaften sowie zur Verständigung über Werte und Normen beitragen." Ebd., S. $45 f$.

53 Thießen 2007, S. 19

54 Vgl. Thießen 2009, S. 374. 
rungsmotivs bestünde dann darin, das gute Ende zu plausibilisieren, etwa im Modus einer individuell ,eingefärbten` Erfolgsgeschichte.

Die erinnerten Geschichten weisen also bestimmte, analysierbare Erzählmuster auf. Deren strukturelle und inhaltliche Beschaffenheit ermöglicht es, Erkenntnisse zu generieren in Bezug auf den erinnerungskulturellen Gehalt, auf die heutige Relevanz für die Zeitzeug_innen sowie in Bezug auf die diesen Geschichten inhärenten, zukünftigen Potenziale.

Die Frage nach der gesellschaftlichen Relevanz von Zeitzeug_innen der Hamburger Sturmflut von 1962 und ihrer erinnerungskulturellen Praktiken kann auch durch einen Rekurs auf aktuelle Debatten über das Ende der Ära der Zeitzeug_innen im Kontext des Holocaust erhellt werden. Aleida Assmann etwa plädiert dafür, „Erinnerungskultur [...] als tragendes Element unserer Zivilgesellschaft auszuweisen." 55 Gleichwohl seien erinnerungskulturelle Praktiken Ausdruck gesellschaftlicher Veränderungen. Assmann identifiziert in diesem Zusammenhang Prämissen einer, wie sie es nennt, „neuen Erinnerungskultur“. ${ }^{56}$ Eine damit einhergehende, neue Wertschätzung von Zeitzeug_innen, wie Assmann sie fordert, möchte auch diese Untersuchung stärken.

Zunächst rekurriert Assmann als Ausgangspunkt ihrer Argumentation auf die bereits bestehende Übereinkunft darüber, dass „Erinnern [...] eine zentrale Form menschlicher Selbstvergewisserung und Orientierung in Raum und Zeit" sei. ${ }^{57}$ Das mittlerweile breite gesellschaftliche Interesse werde nun thematisiert anhand von „individuellen und inoffiziellen Erinnerungen wie Einzelschicksalen, Familiengedächtnissen, Zeitzeugen, Hinterlassenschaften von Tagebüchern, Briefen und Fotos sowie Fragen transgenerationeller Weitergabe“ .58

Des Weiteren spielt, wie hier bereits mit Thießen und Welzer argumentiert, auch für Assmann die konstruktive Kraft der Vergegenwärtigung eine zentrale, neue Rolle: „Erinnern ist Vergegenwärtigung von Vergangenheit. ,Vergegenwärtigung' bedeutet: in der Gegenwart und für die Gegenwart." ${ }^{59}$ Zeitzeug_innen nutzen diese Praxis, und das ist das „Neue“ für Assmann, um sich ihrer Geschichte zu „vergewissern, ihre Eigenart [zu] markieren, ihr Selbstbewusstsein [zu] stärken und Orientierung für die Zukunft [zu] gewinnen." ${ }^{60}$ Ihr Verweis auf die Zukunft fundiert die hier bereits dargelegte, erweiterte Perspektivisierung von Erinnerung. Drittens stellt

\footnotetext{
55 Assmann, Aleida: Das neue Unbehagen an der Erinnerungskultur. München 2013, S. $14 \mathrm{f}$.

56 Ebd., S. 204ff.

57 Vgl. ebd., S. 204.

58 Ebd.

59 Ebd., S. 205. Hervorhebung im Original.

60 Ebd. Assmann führt in Bezug auf erinnerte Vergangenheit aus: „vielmehr greift das, was von ihr ausgewählt und aufgerufen wird, unmittelbar in die Gegenwart ein, um Identitäten zu konstruieren, Einstellungen herbeizuführen, Motivationen zu stimulieren, Handlungen zu ermöglichen, Entscheidungen zu beeinflussen.“ Ebd., S. 206. Diese Erinnerungsleistung ließe sich demgemäß als „Geschichtsbewusstsein“ im Sinne Rüsens bezeichnen.
} 
Assmann heraus, dass „Erinnern [...] der Darstellung [bedarf].“ Die Repräsentationen von Ereignissen seien „niemals einfache Abbildungen, sondern immer schon Modellierungen, Deutungen, Konstruktionen dessen, worauf Bezug genommen“ werde. ${ }^{61}$ Diese, Erinnerungskulturen inhärente, Nähe zu „Medien, Gattungen und Darstellungsformaten “ ermögliche für eine Untersuchung (selbst-)reflexive Rückbezüge bei der Auseinandersetzung mit Vergangenheit und deren Vergegenwärtigung. Assmann hebt viertens als das „Neue“ in Bezug auf Erinnerungskultur „den ethischen Charakter dieses Vergangenheitsbezuges " hervor. ${ }^{62}$ Die vormaligen, als Opfer titulierten und adressierten Menschen erlangten ihren Status als Subjekt zurück, indem sie erinnern, „was man lieber vergessen möchte“ und widersetzen sich somit einem grundlegenden „anthropologischen oder identitätssichernden Bedürfnis“ ${ }^{63}$ Als wesentlichen Motor hierfür macht Assmann „vergemeinschaftete [...] Erinnerungen [aus], die dazu angetan sind, die Leiden der Opfer anzuerkennen und ihre Geschichte in die Erinnerung mit aufzunehmen. " turen stärkend auf Gruppenidentitäten, indem sie ihre Werte bestätigen sowie deren Selbstbewusstsein und Handlungsmacht stützen können. ${ }^{65}$

Die von mir interviewten Zeitzeug_innen sind keine homogene Gruppe und sollen auch nicht als solche interpretiert werden, auch wenn ihre Erinnerungserzählungen durchaus Ähnlichkeiten aufweisen, wie noch ausgeführt wird. Vielmehr entspreche ich einer bereits seit Ende der 1990er Jahre laut gewordenen Forderung, „das kollektive Gedächtnis so [zu] konzipieren und [zu] erforschen, dass seine jeweiligen empirischen Träger sichtbar bleiben ". ${ }^{66}$ Es ist beabsichtigt, diesen integrativen Ansatz durch das hier produzierte Wissensformat Film deutlich zu machen, um so dem Zusammenwirken individueller Erinnerung und sozialer Rahmungen Rechnung zu tragen. ${ }^{67}$

Wie oben dargelegt, werden die Protagonist_innen als Erinnerungsakteur_innen interpretiert. Daher ist es das Anliegen des Films Flut 1962 - Erinnern. Gedenken, Erzählen. die drei „per definitionem "68 miteinander in Relation stehenden Zeitmodi

$61 \quad$ Vgl ebd., S. 206.

62 Vgl. Assmann 2013, S. $207 \mathrm{f}$.

63 Als ein Beispiel für die Umdeutung der (eigenen) Opfer-Rolle kann mittlerweile die (Selbst-)

Bezeichnung, Überlebende_r' (survivor) angesehen werden.

64 Assmann 2013, S. 208f.

65 Ebd., S. 32.

66 Vgl. Pohl 2010, S. 85.

67 „Eine Erinnerung ist um so reicher, je größer die Anzahl jener Rahmen ist, in deren Schnittpunkt sie auftaucht, und die sich in der Tat kreuzen und teilweise gegenseitig decken." Halbwachs, Maurice: Das Gedächtnis und seine sozialen Bedingungen. Frankfurt a. M. 1985 (1925) (abgekürzt: 1985a). Halbwachs expliziert in diesem Zusammenhang bestimmte „Konventionssysteme“, ohne die „[e] in soziales Leben nicht vorstellbar“ (S. 367) wäre. Diese „Bezugsrahmen des Kollektivgedächtnisses" sind für ihn Zeit, Raum sowie weitere Ordnungsstrukturen. Vgl. ebd., S. 368.

68 Welzer, Harald: Vom Zeit- zum Zukunftszeugen. Vorschläge zur Modernisierung der Erinnerungskultur. In: Sabrow, Martin/Frei, Norbert (Hg.): Die Geburt des Zeitzeugen nach 1945. 
des autobiografischen Erzählens - Vergangenheit, Gegenwart, Zukunft - im Kontext von Erinnerungspraktiken darzustellen: auf die Gegenwart verweisen heutige Filmaufnahmen, also die Interviewausschnitte sowie lokale Außenaufnahmen, auf die Vergangenheit wird mittels zeitgenössischer Fotografien und Filmaufnahmen rekurriert. Der dritte Zeitmodus, die Zukunft, wird auf der heutigen sprachlichen Ebene integriert, durch Interviewaussagen zu Reflexionen sowohl über die zukünftige Relevanz erinnerungskultureller Praktiken für nachfolgende Generationen als auch über Hochwasserrisiken durch erneut auftretende Sturmfluten.

\subsection{Erfahrungen als gemeinschaftliche Erzählung}

In der Erhebungsphase der Interviews waren mir die vordergründig großen strukturellen und inhaltlichen Ähnlichkeiten in den Erinnerungserzählungen aufgefallen. Ich hatte den Eindruck, dass sich die Befragten innerhalb ihrer Geschichten an überindividuellen, verinnerlichten Leitmotiven und Erzählmustern orientieren. Dies betraf insbesondere die in der ersten Phase der Interviews erfragte Haupterzählung über die damaligen Ereignisse. Dies erstaunte mich, haben die Menschen doch de facto unterschiedliche Erfahrungen in jener Nacht vom 16. auf den 17. Februar 1962 und während der darauffolgenden Tage gemacht, etwa weil sie an verschiedenen Orten mit unterschiedlichen Auswirkungen durch die Deichbrüche konfrontiert waren: frierend auf dem Dach ihres ebenerdigen Hauses in einer Behelfsheimsiedlung ausharrend, also unmittelbar umgeben von Wassermassen, oder in dem oberen Stockwerk eines Mehrfamilienhauses. Und auch das damalige Lebensalter differenziert sicherlich die individuellen Erfahrungen aus. Dennoch schien es mir nach Erhebung der ersten Interviews fast so, als ob ich nun Bestandteile der Haupterzählungen der weiteren Interviewpartner_innen zumindest teilweise würde antizipieren können.

Eine weitere Auffälligkeit bestand darin, dass nahezu alle Interviewpartner_innen ein Repertoire an zeitgenössischen Quellen zu dem Ereignis besaßen, insbesondere Amateurfotografien, die häufig selbst oder von Nachbarn aufgenommen worden waren, sowie Publikationen, insbesondere den Sonderdruck des Hamburger Abendblatts vom März 1962. ${ }^{69}$ Diese Materialen waren für den Interviewtermin herausgesucht und bereitgelegt worden.

Eine dritte Auffälligkeit war die Selbstdarstellung der - ich nenne sie in diesem Zusammenhang explizit - Zeitzeug_innen, um hier ihren performativen Status als Erinnerungsakteur_innen hervorzuheben. ${ }^{70}$ Alle Befragten, auch diejenigen, die bislang keine Interviewerfahrung hatten, waren in ihrem Auftreten und in ihren

Göttingen 2012, S. 33-48, S. 34.

69 Hamburger Abendblatt: Das war die große Flut. Chronik und Dokumentation der bitteren Tage im Februar 1962. Sonderdruck. Hamburg 1962.

70 Zur Figur des Zeitzeugen als Phänomen der öffentlichen Erinnerungskultur siehe Sabrow, Martin/Frei, Norbert (Hg.): Die Geburt des Zeitzeugen nach 1945. Göttingen 2012. 
Ausführungen eloquent und machten auf mich einen insgesamt souveränen Eindruck. Es bestand wenig Scheu oder Zurückhaltung, von den Erlebnissen zu erzählen. Ich fragte mich, ob die Interviewpartner_innen mir gegenüber fast so etwas wie eine aktuell virulente Vermittlungsabsicht verfolgten, da sie sich im Interview weniger, wie von mir erwartet, auf eine mentale Zeitreise in die Vergangenheit begaben. Offensichtlich lagen hier nicht die „erinnerungskulturellen Voraussetzungen “71 vor, die, wie Welzer anmerkt, zu einer zeitlich ausschließlich rückwärtsgewandten Perspektivisierung geführt hätten.

Die insgesamt selbstbewusste Ausstrahlung der Befragten vermittelte mir zudem den Eindruck, dass die damaligen Ereignisse mittlerweile bewältigt wurden. Auch wenn in den Geschichten durchaus eine emotionale Involviertheit spürbar war, so wurde insbesondere durch die erzählerischen Fertigkeiten deutlich, dass das Erlebte durchdacht, beurteilt und in das heutige Leben integriert worden ist. Hier knüpft eine volkskundlich-kulturanthropologische Erzählforschung an, die bewusstseinsanalytisch „nach den historischen Ursprüngen der Gegenwart" fragt. ${ }^{72}$ Dieser Zugang untersucht erstens die Erzählmuster, an denen sich Menschen in ihren Geschichten orientieren. Dabei interessieren mich zweitens die leitmotivisch orientierten Darstellungen, die erst durch die Vergleichbarkeit mit den Erzählungen anderer zutage treten.

Das Erzählen - gemeint ist damit also sowohl das, was gesagt wird, als auch wie es gesagt wird - interpretiere ich als soziale Handlung, die sowohl auf gesellschaftliche Prozesse ausgerichtet ist als auch eine zwischenmenschliche Performanz in der konkreten Interviewsituation darstellt. ${ }^{73}$ Gleichwohl dient das Erzählen auch einer gesprächsimmanenten, hier im Folgenden grundiert durch die Psychologin Gabriele Lucius-Hoene und den Germanisten Arnulf Deppermann, „Rekonstruktion narrativer Identität" ${ }^{74}$ Identität wird auch in dieser Untersuchung interpretiert als Selbstverortung innerhalb einer Gruppe, also als ein Konzept prozessualer Auseinandersetzung des Individuums mit sich selbst und mit anderen. In Krisensituationen, wie der Hamburger Sturmflut von 1962, in denen Gruppen herausgefordert werden, gerät auch die eigene Selbstsicherheit aus dem Gleichgewicht. Zur Rückgewinnung von Normalität, gewissermaßen als Ordnungsstruktur, kommt dem verbalen Aus-

71 Welzer 2012, S. 34.

72 Vgl. Lehmann 2007, S. 138.

73 Die Untersuchung von performativen Praktiken in der Interviewsituation gilt als Schwerpunkt insbesondere der US-amerikanischen Folklore Studies. Dabei wird, wie Stefan Beck herausstellt, die „Aufmerksamkeit insbesondere auf die je individuelle, kreative und spielerische Praxis der Akteure in Interaktionen gerichtet“. Beck, Stefan: Die Bedeutung der Materialität der Alltagsdinge. Anmerkungen zu den Chancen einer wissenschaftstheoretisch informierten Integration von Symbol- und Sachforschung. In: Brednich, Rolf Wilhelm/Schmitt, Heinz (Hg.): Symbole. Zur Bedeutung der Zeichen in der Kultur. 30. Deutscher Volkskundekongreß in Karlsruhe vom 25. bis 29. September 1995. Münster u. a. 1997, S. 175-186, S. 183.

74 Vgl. Lucius-Hoene, Gabriele/Deppermann, Arnulf: Rekonstruktion narrativer Identität. Ein Arbeitsbuch zur Analyse narrativer Interviews. 2. Auflage. Wiesbaden 2004. 
tausch, also dem „Reden über Erfahrung “75 als „grundlegendste Katastrophenhilfe“, eine große Relevanz zu, wie hier bereits mit Bendix ausgeführt wurde. ${ }^{76}$

Die sprachlichen Modi der erhobenen Erinnerungserzählungen stehen also im Mittelpunkt dieser Untersuchung. Dabei verbindet sie inter- und transdisziplinäre Zugangsweisen der Volkskunde/Kulturanthropologie mit einem „pragmatischen Funktionalismus der Linguistik“".77 Die verschiedenen methodischen Ansätze zur Analyse narrativer Interviews unterscheiden sich ,hinsichtlich ihrer erkenntnistheoretischen Grundlagen "78, allerdings erscheint ein solcherart kombiniertes analytisches Vorgehen sinnvoll, wie bereits andere Forschungsarbeiten bestätigen. ${ }^{79}$

Sowohl individuelle Haltungen als auch gesellschaftlich verankerte Sinnstrukturen, die in den Erzählungen deutlich werden, sind auch hier von Interesse. Im Folgenden stehen daher Zugänge einer kulturwissenschaftlichen Erzählforschung als Bewusstseinsanalyse im Mittelpunkt. Dafür werden Forschungsstände und wesentliche Forschungsperspektiven im Kontext des Untersuchungsthemas expliziert und reflektiert.

$75 \quad$ Lehmann 2007.

76 Bendix 1996, S. 170.

77 Vgl. Herlyn, Gerrit: Systematik oder Intuition? Zur kulturanalytischen Praxis der Auswertung qualitativer Interviews. In: Johler, Reinhard u. a. (Hg.): Kultur_Kultur. Denken. Forschen. Darstellen. 38. Kongress der Deutschen Gesellschaft für Volkskunde in Tübingen vom 21.-24. September 2011. Münster u. a. 2013, S. 485-491, S. 486f. Herlyn setzt sich darin kritisch auch mit dem Analyseansatz von Lucius-Hoene und Deppermann auseinander. Siehe hierzu auch Sutter, Ove: Erzählte Prekarität. Autobiographische Verhandlungen von Arbeit und Leben im Postfordismus. Frankfurt a. M./New York 2013, S. 87.

78 Kofler, Alexandra: Erzählen über Liebe. Die Konstruktion von Identität in autobiografischen Interviews. Frankfurt a. M./New York 2012, S. 91.

79 Siehe hierzu Sutter 2013; Herlyn, Gerrit: Computer im Alltag - Computer als Alltag. Erzählstrategien und biographische Deutungen im Veralltäglichungsprozess von Technik. Hamburg 2008; Kofler 2012; Leikauf, Roland: „Welcome to My Bunker“ - Vietnamkriegserfahrungen im Internet. Bielefeld 2016. Sutter etwa nutzt diesen Zugang für seine Untersuchung als für ihn „zentrale Referenz" unter Verweis darauf, dass Lucius-Hoene und Deppermann „das eher mikroanalytisch ausgerichtete funktional-pragmatische Konzept auf die Analyse jener autobiographischen Großerzählungen übertragen haben, die durch narrative Interviews erhoben werden." Sutter 2013, S. 90. 



\section{Erzählforschung über die Hamburger Sturmflut von 1962 mit Film - Forschungsstand und -perspektiven}

Als ,Naturkatastrophen' rezipierte Extremereignisse gelten im deutschsprachigen Raum primär als Forschungsgebiete der Soziologie und der Geografie - seit einigen Jahren insbesondere im Kontext von Untersuchungen zum Phänomen des Klimawandels. ${ }^{80}$ Die Geschichtswissenschaft fokussierte lange Zeit anhand historisch-archivalischer Zugänge vornehmlich auf katastrophale Geschehnisse für die Zeit bis in das 19. Jahrhundert. ${ }^{81}$

Die Katastrophenforschung der Volkskunde/Kulturanthropologie beschäftigt sich mit der Wahrnehmung, Deutung und Kommunikation von katastrophalen Ereignissen. Diesen Umgang mit einem Extremereignis analysiert sie zudem im Kontext von Bewältigungsstrategien und in seiner Abhängigkeit von gesellschaftlichen Konventionen und Handlungsfeldern. ${ }^{82}$ Individuelles Erfahrungswissen wurde indes bisher

${ }^{80}$ Siehe hierzu z. B. Voss, Martin (Hg.): Der Klimawandel. Sozialwissenschaftliche Perspektiven. Wiesbaden 2010.

81 Siehe hierzu z. B. Borst 1981; Groh, Dieter/Kempe, Michael/Mauelshagen, Franz: Einleitung. Naturkatastrophen - wahrgenommen, gedeutet, dargestellt. In: Dies. (Hg.): Naturkatastrophen. Beiträge zu ihrer Deutung, Wahrnehmung und Darstellung in Text und Bild von der Antike bis ins 20. Jahrhundert. Tübingen 2003, S. 11-33.

82 Vgl. Douglas, Mary: Risk and Blame. Essays in Cultural Theory. London/New York 1992, insbesondere S. 38-54. 
kaum qualitativ erfasst und analysiert. Seit einigen Jahren wird diesem Forschungsdefizit entgegengewirkt, insbesondere durch Untersuchungen von Bernd Rieken ${ }^{83}$, fokussierend auf Perspektiven der Erzählforschung als Bewusstseinsanalyse sowie durch das Tübinger Teilprojekt im Sonderforschungsbereich ,Bedrohte Ordnungen'. Diese Forschungszugänge werden nachfolgend in Kapitel 2.3 weiter ausgeführt.

Erzählforschung als Bewusstseinsanalyse stellt, wie Lehmann für dieses Forschungskonzept hervorhebt, „die Menschen, ihre Erfahrungen und Wünsche, Welt- und Wirklichkeitsbilder" in seinen Mittelpunkt. ${ }^{84}$ Von der erzählerischen Gegenwart ausgehend werden primär gesprochene Texte analysiert und interpretiert. Der Einbezug von schriftlichen Selbstzeugnissen, Fotografien und anderen materiellen Gütern kann zusätzliche Erkenntnisse hervorbringen.

Anknüpfend an die volkskundliche Fachtradition der Sagen- und Märchenforschung steht hier nicht - wie zumeist in der Oral History - der historische Quellenwert von Erinnerungen im Fokus des Interesses, sondern die funktionale Bedeutung der Erzählungen für den Einzelnen und für Gruppen, respektive für eine Gesellschaft, innerhalb derer diese Erzählungen platziert sind und rezipiert werden. ${ }^{85}$ Dabei ist davon auszugehen, dass diese Erzählungen nicht allein auf individueller Erfahrung gründen, sondern in ihnen „ein Wissen aus zweiter Hand“86 inkorporiert ist. Das Gedächtnis kombiniert „unentwirrbar “87 Gelesenes und Aufgeschnapptes aus verschiedenen Publikationen, Fernsehsendungen und aus den Geschichten anderer Menschen. Um analytisch der daraus folgenden Unmöglichkeit, eine Erzählung ,entwirren' zu können, zu begegnen, interpretiere ich das Zusammenwirken von originären eigenen Erfahrungen mit von Anderen übernommenen Inhalten bewusst als in seiner Gesamtheit subjektive Erinnerungs- respektive Erzählleistung, also als ein „Amalgam“" ${ }^{88}$. Letztlich für eine Analyse zugänglich ist nur eine solcherart verwobene "nach außen gerichtete Selbstdarstellung ". ${ }^{89}$

Diese induktiv ausgerichtete Untersuchung über die Hamburger Sturmflut von 1962 fragt nach den Bedeutungen des Erzählens und weist dem Sprechen eine sowohl individuelle als auch soziale Funktion zu. Auch Silke Meyer weist auf dieses Zusammenwirken hin, wenn sie Erzählungen sowohl ,als individuelle Akte der Sinnstiftung" als auch „als Träger von intersubjektiven Werthaltungen und Hand-

83 Siehe hierzu Rieken, Bernd: Schatten über Galtür? Ein Beitrag zur Katastrophenforschung. Münster 2010; Rieken 2016.

84 Vgl. Lehmann 2007 (Klappentext).

85 Zur Kompatibilität der volkskundlich-kulturanthropologischen Erzählforschung mit Ansätzen der Oral History siehe Leikauf 2016, S. $44 \mathrm{ff}$.

86 Lehmann 2007, S. 36.

87 Ebd.

88 Assmann definiert die Leistung des Gedächtnisses als ein „Amalgam aus der Geschichte als Erzählung und der Geschichte als Ereignis“. Assmann 2003, S. 35.

89 Vgl. Leikauf 2016, S. 39. 
lungsmaximen“ interpretiert. ${ }^{90}$ Oder wie Klara Löffler formuliert: „Erzählen ist die Brücke über den Dualismus zwischen persönlicher und sozialer Identität. “91 Sie hebt zudem eine weitere Bedeutung und Folge sprachlichen Handelns hervor, nämlich die adressierte Person mit in die Geschichte einzubeziehen. ${ }^{92}$ Auf die Funktion der Erzeugung eines gemeinsamen Vorstellungsraumes im Erzählen weist auch Hans Joachim Schröder hin. ${ }^{93}$ Dieser gründet sich auf bestimmte inhaltliche und strukturelle Erzählkonventionen, die mir als Interviewerin zuvor bekannt waren. Die Befragten rekurrieren auf eine, wie ich sie im Folgenden nenne, in der Gesellschaft verankerte Meistererzählung über das damalige Geschehen. Diese wird hier in ihrem strukturellen und inhaltlichen Gehalt als analytische Referenz interpretiert, denn die erhobenen Interviews sind maßgeblich durch diese Meistererzählung bestimmt, wie ich bereits oben anhand der antizipierbaren Ähnlichkeiten angedeutet habe. In dieser Erzählung sind bestimmte Leitmotive verankert, die inhaltliche Orientierungsmarker für die Erzählenden anbieten sowie Erzählmuster, die eine die Erlebnisse evaluierende Botschaft bereitstellen.

\subsection{Die Hamburger Sturmflut von 1962 als Forschungsfeld: bisherige Zugänge und Untersuchungen}

Die Hamburger Sturmflut von 1962 gilt bis in die heutige Zeit als Bestandteil des kollektiven Gedächtnisses der Stadt Hamburg und wird deutschlandweit erinnert. Es hat sich eine gesellschaftliche Übereinkunft über die Erzählweisen und -inhalte herausgebildet. Diese Darstellung möchte ich wie folgt zusammenfassen:

In der Nacht vom 16. auf den 17. Februar 1962 wurde Hamburg von einer bis dahin unvorstellbar schweren Sturmflut heimgesucht. Das orkanartige Sturmtief „Vincinette“ fegte bereits seit mehreren Tagen aus nordwestlicher Richtung über die Nordseeküste hinweg und drückte das Wasser in die Elbmündung hinein. Die Beamten der zuständigen Hamburger Behörden und die Bevölkerung waren unzureichend vorbereitet, auch die Technik versagte. Außerdem waren die Deiche veraltet und finanzielle Mittel für ihre Instandhaltung limitiert. Eine vergleichbare Naturkatastrophe hatte sich zuletzt 1825 ereignet.

Als ab Mitternacht die Deiche überliefen und es im weiteren Verlauf zu über 60 Deichbrüchen kam, war die Bevölkerung davon im Schlaf überrascht

$90 \quad$ Vgl. Meyer, Silke: Was heißt Erzählen? Die Narrationsanalyse als hermeneutische Methode der Europäischen Ethnologie. In: Zeitschrift für Volkskunde 110 (2), 2014, S. 243-267, S. 245.

91 Löffler, Klara: Zurechtgerückt. Der Zweite Weltkrieg als biographischer Stoff. Berlin 1999, S. 70.

92 Ebd., S. 92.

93 Vgl. Schröder, Hans Joachim: Die gestohlenen Jahre. Erzählgeschichten und Geschichtserzählungen im Interview: der Zweite Weltkrieg aus der Sicht ehemaliger Mannschaftssoldaten. Tübingen 1992, hier S. 153ff. Schröder nimmt Bezug auf Jochen Rehbeins Ausführungen zur Unterscheidung zwischen Erzählen und Berichten. Vgl. Rehbein, Jochen: Beschreiben, Berichten und Erzählen. In: Ehlich, Konrad (Hg.): Erzählen in der Schule. Tübingen 1984, S. 67-124. 
worden. In den Hamburger Stadtteilen Cranz und in Neuenfelde, in Francop und auf Finkenwerder, in Waltershof und in Altenwerder bis hinunter nach Moorburg, also insbesondere der Süderelberaum wurde von den Wassermassen überflutet, die ca. 220 Millionen Kubikmeter entsprechen dem 60-fachen Inhalt von Binnen- und Außenalster zusammen. Aber auch nördliche, an der Elbe gelegene Gebiete wie Teile von Billwerder-Moorfleet standen unter Wasser. Am stärksten betroffen war die Elbinsel Wilhelmsburg. Insgesamt war ein Fünftel des Hamburger Stadtgebietes überflutet. Um 3.07 Uhr betrug der Wasserstand am Pegel St. Pauli 5,70 Meter über Normalnull (NN). Zu diesem Zeitpunkt hatte sich bereits der schwerste Deichbruch, am Spreehafen im Norden Wilhelmsburgs, ereignet. Dort kamen in den Behelfsheimsiedlungen über 200 Menschen zu Tode.

Dem damaligen Polizei- und späteren Innensenator Helmut Schmidt war es zu verdanken, dass ab dem 17. Februar unbürokratisch und koordiniert umfassende Hilfsmaßnahmen eingeleitet wurden. Etwa 26.000 Helfer_innen kamen zum Einsatz, davon über 7.000 Bundeswehrsoldaten, mit über 120 Hubschraubern auch von ausländischen Einheiten, wie der US Air Force und der Royal Air Force, des Weiteren 5.000 Polizisten, 3.000 Feuerwehrmänner sowie Angehörige von verschiedenen Hilfsorganisationen. Die betroffenen Gebiete waren über mehrere Tage ohne Wasser-, Gas- und Stromversorgung sowie ohne Telefonverbindung. Die Überflutungen dauerten an einigen Stellen bis zu vier Wochen an. Am 26. Februar 1962 versammelten sich auf dem Hamburger Rathausmarkt rund 150.000 Menschen, um in einer Trauerfeier der Toten zu gedenken. Neben anderen Vertretern der Bundesregierung war auch Bundespräsident Heinrich Lübke anwesend.

Die Sturmflut forderte in Hamburg insgesamt 315 Todesopfer, Tausende von Nutz- und Haustieren ertranken, etwa 100.000 Menschen waren von den Wassermassen eingeschlossen, über 6.000 Behelfsheime wurden zerstört oder schwer beschädigt und fast 10.000 Wohnungen waren monatelang unbewohnbar. Die finanziellen Schäden - inklusive der Produktionsausfälle und zerstörten Gewerbebetriebe - beliefen sich auf rund 1 Milliarde DM.

Es setzte eine damals nicht für möglich gehaltene Welle der Hilfsbereitschaft und Solidarität ein, die durch Sach- und Geldspenden aus dem Inund Ausland ihren Ausdruck fanden. Dieser Gemeinschaftsgeist zeigte sich zudem in der Nachbarschaftshilfe sowie innerfamiliär auch zwischen den Generationen. Die Bewohner_innen der überfluteten Gebiete erhielten bald staatliche finanzielle Unterstützung. Tausende von Kindern, Müttern und älteren Leuten wurden - ebenfalls mit Hilfe von Spenden - zur Erholung verschickt.

Nach der Flut begannen die Verantwortlichen der zuständigen Behörden nach neuesten wassertechnischen Erkenntnissen, Hochwasserschutzanlagen 
zu planen und zu bauen, deren Kosten mit über 500 Millionen DM veranschlagt wurden. Ein Katastrophenschutzplan für Hamburg folgte. ${ }^{94}$

Der hier dargelegte, chronologisch aufgebaute Bericht über die damaligen Ereignisse und seine Folgen gibt stringent und quantifizierend die Geschehnisse wieder und formuliert deren Abfolge und Bedingtheiten. ${ }^{95}$ Durch seine Nüchternheit unterscheidet sich dieser Bericht von den „Vorstellungsbilder“ evozierenden Erinnerungserzählungen, die demgemäß weit mehr über eine „Affinität zur Bildlichkeit“ verfügen, wie sich anhand der Interviewanalyse bestätigen lässt. ${ }^{96}$

Diese sachliche Schilderung hat mit seinem strukturgebenden Rahmen Einfluss auf die individuellen Geschichten. Sie sind ebenfalls von „textuelle[r] Kohärenz“ geprägt und typisch dafür, wie Mitglieder einer modernen, westlichen Gesellschaft ihre kollektiven Gedächtnisse organisieren. ${ }^{97}$ Der Kulturwissenschaftler Mathias Berek spricht von einer priorisierenden kollektiven Überformung, wenn Erzählungen „sich nicht nur auf die Wiedergabe persönlicher Erlebnisse beschränken, sondern eine Bedeutung innerhalb der jeweiligen Erinnerungskultur haben ". ${ }^{98}$ Lediglich ein „chronologisches und räumliches Schema“ werde zurückbehalten, um, wie Maurice Halbwachs hervorhebt, „die Totalität der vergangenen Ereignisse [...] zu einem

94 Die Zahlen werden seit 1962 in verschiedenen Berichten wiederholt angeführt, wie etwa in Eismann, Martina/Mierach, Michael: Wenn die Flut kommt ... Erinnerungen an die Katastrophe von 1962 und heutiger Hochwasserschutz. Hg. von der Behörde für Bau und Verkehr Hamburg/Amt für Wasserwirtschaft. Hamburg o. J. (2002) sowie in Hötte, Herbert (Hg.): Die große Flut. Katastrophe. Herausforderung. Perspektiven. Begleitbuch zu den Ausstellungen im Hamburger Rathaus und im Museum für Hamburgische Geschichte aus Anlass des 50. Jahrestages der Sturmflut vom Februar 1962. Hamburg 2012. Eine eigenständige Archivrecherche zur Überprüfung der Daten fand im Rahmen dieser Untersuchung nicht statt. Ich gehe davon aus, dass die Größenordnungen stimmig sind. In den meisten Publikationen werden die Todesopfer auf Hamburger Stadtgebiet mit 315 beziffert, diese Zahl wird darin ebenfalls ohne Quellenangabe genannt. Der vom Hamburger Senat berufene Sachverständigenausschuss kam Ende März 1962 zu dem Ergebnis, dass auf Seiten der Bevölkerung 312 Menschen zu Tode kamen sowie fünf Helfer (zwei Bundeswehrsoldaten, je ein Mitarbeiter des Deutschen Roten Kreuzes und des Technischen Hilfswerk sowie ein Angestellter der Verwaltung). Vgl. Bericht des vom Senat der Freien und Hansestadt Hamburg berufenen Sachverständigenausschusses zur Untersuchung des Ablaufs der Flutkatastrophe. Hamburg 1962, S. 53. Dieser Bericht wurde Ende März 1962 veröffentlicht. Die weiteren, noch bis August desselben Jahres geborgenen Toten sind darin nicht aufgeführt. Vgl. Mauch 2015, S. 90.

${ }_{95} \mathrm{Zu}$ den Unterscheidungsmerkmalen zwischen Erzählen und Berichten siehe Lehmann, Albrecht: Erzählstruktur und Lebenslauf. Autobiographische Untersuchungen. Frankfurt a. M./New York 1983, S. 64ff.; siehe Lucius-Hoene/Deppermann 2004, S. 143ff.

96 Vgl. Vgl. Gerndt, Helge: Mit Bildern erzählen. In: Brednich, Rolf Wilhelm (Hg.): Erzählkultur. Beiträge zur kulturwissenschaftlichen Erzählforschung. Hans-Jörg Uther zum 65. Geburtstag. Berlin/New York 2009, S. 309-325, S. 322 f.

97 Vgl. Pethes, Nicolas: Kulturwissenschaftliche Gedächtnistheorien zur Einführung. Hamburg 2008, S. 6, mit Bezug auf Assmann 1999, S. 130-142.

98 Vgl. Berek, Mathias: Kollektives Gedächtnis und die gesellschaftliche Konstruktion der Wirklichkeit. Eine Theorie der Erinnerungskulturen. Wiesbaden 2009, S. 90. 
einzigen Bild zusammen[zu]stellen ". ${ }^{99}$ Der Historiker Paul Nolte weist in diesem Zusammenhang diesen Synthesen eine Orientierung gebende Funktion zu. Mehr noch sind „Meistererzählungen“ für ihn nicht nur „epistemische Deutungsmuster“, sondern in ihrer textuellen Tiefenstruktur und in ihrer narrativen Realisierung sei gleichsam ein „Anspruch auf autoritative Geltung“ eingeschrieben. ${ }^{100}$ Seine Ausführungen stehen in einem hier relevanten Spannungsverhältnis zu der Interpretation von Halbwachs, der von der Voraussetzung ausgeht, dass man die Ereignisse „vom Gedächtnis jener Gruppen löst, die sie in Erinnerung behielten“". ${ }^{101}$ Hieraus ließe sich folgern, dass sich auch die von mir befragten Zeitzeug_innen sowohl an überindividuell geprägten Vorlagen orientieren als auch subjektive Erfahrungen nach wie vor Bestandteil der Erzählungen sind. Daher beziehe ich mich im Folgenden auf den oben angeführten Bericht als die in der Gesellschaft verankerte Meistererzählung über die Hamburger Sturmflut von 1962. Die individuellen, in Interviews erhobenen Erzählungen bezeichne ich dem gegenüber im Folgenden als subjektbezogene bzw. persönliche Masternarrationen. Der obige Bericht hat also großen Einfluss auf die individuellen Erinnerungsgeschichten, wie auch die Analyse in Kapitel 4.2 zeigen wird. Dies gilt ebenso für die seither publizierten Ereignisdarstellungen.

\section{Graue Literatur}

Die Hamburger Sturmflut von 1962 stand bis vor wenigen Jahren nur selten im Zentrum wissenschaftlicher Betrachtung. Vielmehr sind seit 1962 kontinuierlich unterschiedliche Publikationen sogenannter, grauer Literatur' erschienen, Erfahrungsschilderungen etwa, die, kombiniert mit quantifizierenden Darstellungen der Ereignisabläufe, herausgegeben von lokalen und städtischen Institutionen, einen zugleich bilanzierenden und teilweise durchaus auch moralisierenden Duktus aufweisen. ${ }^{102}$ Ähnlich angelegt sind etwa aus Anlass des 40. Jahrestages 2002 im Eigenverlag publizierten Erfahrungsberichte von Zeitzeug_innen, illustriert mit eigenen Fotografien. ${ }^{103}$ Des Weiteren sind Tatsachenromane zu nennen, die fiktive Personen und ihr dramatisches Schicksal während der Flutnacht in den Mittelpunkt

99 Vgl. Halbwachs 1985b, S. 73.

100 Vgl. Nolte, Paul: Von Glück und Streit, Lernen und Stabilität. Historiografische Meistererzählungen deutscher Demokratie. In: Hertfelder, Thomas/Lappenküper, Ulrich/Lillteicher, Jürgen (Hg.): Erinnern an Demokratie in Deutschland. Demokratiegeschichte in Museen und Erinnerungsstätten der Bundesrepublik. Göttingen 2016, S. 121-137, S. $124 f$.

101 Vgl. Halbwachs 1985b, S. 73.

102 Siehe hierzu Wilhelmsburger Zeitung (Hg.): Der 17. Februar 1962. Hamburg o. J. (ca. 1962); Keesenberg, Hermann: Die Wilhelmsburger Sturmflutkatastrophe 1962 aus heimatgeschichtlicher Sicht. Hg. von der Wilhelmsburger Zeitung. Hamburg o. J. (ca. 1962); Wilhelmsburg 1962. Hg. von den Wilhelmsburger Heimatvereinen. Hamburg 1962; Die große Flut in Hamburg. Eine Chronik der Katastrophe vom Februar 1962. Im Auftrag und in Zusammenarbeit mit der Schulbehörde zusammengestellt von Hans Bütow. Hamburg o. J. (ca. 1963).

103 Siehe hierzu Mohr, Henry/Köpke, Rudolf (Hg.): Die große Flut. Augenzeugen berichten über ihre Erlebnisse vom 16. auf den 17. Februar 1962. Hamburg 2003. 
stellen. ${ }^{104}$ Darüber hinaus wurden Begleitpublikationen zum 40. und zum 50. Jahrestag im Kontext von behördlich organisierten Ausstellungen veröffentlicht, die in ihrer Grundaussage primär auf die Darstellung von heutigen Hochwasserschutzmaßnahmen ausgerichtet sind. Die Kombination aus Zeitzeug_inneninterviews, chronologischen Darstellungen der Abläufe und zeitgenössischen Fotografien kommt darin eine die Gesamtaussageabsicht der Publikationen stützende Funktion zu. Das Ereignis dient darin vornehmlich als mahnende, aber explizit vergangene Referenz. ${ }^{105}$ Publikationen, die an Jugendliche adressiert sind bzw. deren Sichtweisen thematisieren, werden ebenfalls bis in die heutige Zeit veröffentlicht. ${ }^{106}$ Sie verfolgen einen pädagogischen Ansatz bzw. wurden sie, unter Mitwirkung von Zeitzeug_innen, als Schüler_innenprojekte von Mädchen und Jungen verfasst, die mittlerweile der Enkel- und Urenkelgeneration angehören. ${ }^{107}$

\section{Wissenschaftliche Publikationen}

In wissenschaftlichen Publikationen kommt der Hamburger Sturmflut von 1962 häufig eine Referenzfunktion zu. Dies gilt etwa bei Forschungsprojekten der Geografie sowie der Stadt- und Raumplanung. Basierend auf quantifizierenden Auswertungen fokussieren diese im Kontext des Klimawandels auf Vorhersagemöglichkeiten für Hamburg bzw. auf Fragen des Risikomanagements. ${ }^{108}$

Die Geschehnisse werden bislang hauptsächlich aus geschichtswissenschaftlicher Perspektive untersucht: Ein vielfach zitierter Aufsatz von Jens Ivo Engels thematisiert umfassend aus umwelthistorischer Perspektive den Wandel des Naturbildes in der Bundesrepublik aufgrund von „Naturkatastrophen“ und die Bedeutung, die der

104 Siehe hierzu Herlin, Hans: Die Sturmflut. Nordseeküste und Hamburg im Februar 1962. Hamburg 1982. (Hg. zusammen mit dem Hamburger Abendblatt); Herlin, Hans: Die Sturmflut. Nordseeküste und Hamburg im Februar 1962. Hamburg 1997. (Neuauflage mit stark reduzierter Anzahl an Fotografien); Schuller, Alexander: Sturmflut über Hamburg. Die Nacht, in der die Stadt ertrank. Ein Tatsachenroman. München 2006.

105 Siehe hierzu Eismann/Mierach 2002; Hötte 2012.

106 Siehe hierzu Boie-Eyer, Ursula: Die Hamburger Sturmflut. Göttingen o. J. (ca. 1963); Boie, Kirsten: Ringel, Rangel, Rosen. Hamburg 2010.

107 Siehe hierzu: Geschichtswerkstatt Wilhelmsburg \& Hafen/Pauw Literaturmanagement: Als der Deich brach. Sturmflut - Wortflut. Jugendliche erzählen. Hamburg 2012; „Wir haben viel Glück gehabt“. Lebensgeschichten zur Sturmflut 1962. Hg. von der Stadtteilschule Stellingen/ Ida Ehre Schule. Hamburg 2016 (inkl. DVD eines Interviews mit Helmut Schmidt).

108 Vgl. Gönnert, Gabriele: Sturmfluten in der Elbe - Das Hochwasser- und Bemessungskonzept für Hamburg. In: Ratter, Beate (Hg.): Küste und Klima. Hamburger Symposium Geographie, Bd. 1. Hamburg 2009, S. 23-33; Ratter, Beate/Kruse, Nicole: Klimawandel und Wahrnehmung. Risiko und Risikobewusstsein in Hamburg. In: Böhner, Jürgen/Ratter, Beate (Hg.): Klimawandel und Klimawirkung. Hamburger Symposium Geographie, Bd. 2. Hamburg 2010, S. 119-137; vgl. Knieling, Jörg/Schaerffer, Mareike/Tressl, Stephan: Klimawandel und Raumplanung. Flächen- und Risikomanagement überschwemmungsgefährdeter Gebiete am Beispiel der Hamburger Elbinsel. Hamburg 2009. 
Sturmflut von 1962 hierbei zukommt. ${ }^{109}$ Als Untersuchungsgrundlage dienen Engels Tages- und Wochenpresse, Verlautbarungen der Landes- und Bundesregierung sowie Sitzungsprotokolle der Parlamente und Ausschüsse. Aus stadthistorischer Perspektive untersucht Dieter Schott Interpretationsnarrative politischer Akteur_innen. ${ }^{110}$ Schott kommt in Bezug auf die Hamburger Sturmflut von 1962 zu dem Ergebnis, dass es damals von politischer Seite als opportun galt, vor dem Hintergrund der Erfahrungen des Zweiten Weltkrieges, eine Schicksalsgemeinschaft zu implementieren mit dem Ziel, ein Bewusstsein lokaler und nationaler Solidarität zu erzeugen. Die Ergebnisse von Engels und Schott aufnehmend, ordnet Michael Kempe das Ereignis in den Kontext historischer Sturmfluten an der Nordseeküste ein und fokussiert dabei auf erinnerungskulturelle Besonderheiten. Er legt dar - hierbei bezieht er sich auf Gedenkreden der nachfolgenden 25 Jahre -, dass von institutioneller Seite unverändert auf die identitätsstiftenden Funktionen von Solidarität und Hilfsbereitschaft rekurriert wurde. ${ }^{111}$

Die bisherigen volkskundlich-kulturanthropologischen Untersuchungen haben eine mentalitätsgeschichtliche Ausrichtung. Die Hamburger Sturmflut von 1962 steht dabei sowohl bei Norbert Fischer als auch bei Bernd Rieken nicht im primären Fokus des Interesses. Fischer widmet sich aus sozialhistorischer Perspektive den gesellschaftlichen Bedingungen an der Nordseeküste und im Niederelberaum im Kontext des Deichbaus in der Länderregion Kehdingen-Oste. ${ }^{112}$ Und in seinen Untersuchungen zu maritimen Gedächtnislandschaften bezieht er vergleichend die Hamburger Ereignisse bzw. dortige erinnerungskulturelle Artefakte in seine analytischen Betrachtungen mit ein. ${ }^{113}$ Rieken nimmt sich in seiner Längsschnittuntersuchung zur Geschichte der Sturmfluten an der Nordsee in einem Kapitel auch den

109 Vgl. Engels, Jens Ivo: Vom Subjekt zum Objekt. Naturbild und Naturkatastrophen in der Geschichte der Bundesrepublik Deutschland. In: Groh, Dieter/Kempe, Michael/Mauelshagen, Franz (Hg.): Naturkatastrophen. Beiträge zu ihrer Deutung, Wahrnehmung und Darstellung in Text und Bild von der Antike bis ins 20. Jahrhundert. Tübingen 2003, S. 119-142.

110 Vgl. Schott, Dieter: One City - Three Catastrophes: Hamburg from the Great Fire 1842 to the Great Flood 1962. In: Massard-Guilbaud, Geneviève/Platt, Harold L./Ders. (Hg.): Cities and Catastrophes. Coping with Emergency in European History. Frankfurt a. M. u. a. 2002, S. 185204.

111 Vgl. Kempe, Michael: ,Mind the Next Flood!' Memories of Natural Disasters in Northern Germany from the Sixteenth Century to the Present. In: The Medieval History Journal 10 (1/2), 2007, S. 327-354.

112 Vgl. Fischer, Norbert: Der wilde und der gezähmte Fluss - Zur Geschichte der Deiche an der Oste. Stade 2011 (abgekürzt: 2011c); vgl. Fischer, Norbert: Wassernot und Marschgesellschaft. Zur Geschichte der Deiche in Kehdingen. Stade 2003.

113 Vgl. Fischer, Norbert: Maritime Gedächtniskultur an der Nordseeküste. Adaptionen der Katastrophe. In: Fischer, Ludwig/Reise, Karsten (Hg.): Küstenmentalität und Klimawandel. Küstenwandel als kulturelle und soziale Herausforderung. München 2011, S. 77-95 (abgekürzt 2011b); vgl. Fischer, Norbert: Gedächtnislandschaften in Geschichte und Gegenwart. Kulturwissenschaftliche Studien. Wiesbaden 2016. 
Hamburger Ereignissen von 1962 an und stützt sich hierbei etwa auf den bereits genannten Aufsatz von Engels. ${ }^{114}$ Bislang liegen zwei Aufsätze von mir vor, in denen lokale bzw. geschlechtsspezifische Besonderheiten des Erzählens über die damaligen Geschehnisse im Mittelpunkt stehen. ${ }^{115}$

Der von den Historiker_innen Martina Heßler und Christian Kehrt der Helmut-Schmidt-Universität/Universität der Bundeswehr Hamburg im Jahr 2014 publizierte Sammelband thematisiert die Hamburger Sturmflut von 1962 erstmals umfassend als „multidimensionale[s] Ereignis“ aus umwelt-, technik- und zeitgeschichtlicher Perspektive. ${ }^{116}$ Er ist das Ergebnis einer Tagung, die 2012 zum 50. Gedenkjahr stattgefunden hatte. Heßler und Kehrt interpretieren das Geschehen insgesamt als ein „zeithistorisches Schlüsselereignis“117 in der bundesrepublikanischen Gesellschaft. Auch sie konstatieren, dass es in der Zeitgeschichtsschreibung bisher wenig Beachtung gefunden habe und heben demgegenüber den „engen Zusammenhang mit Kriegserfahrungen, dem Katastrophenschutz und der Geschichte der Bundeswehr" ${ }^{118}$ hervor. Heutige Aktualität und Relevanz des damaligen Ereignisses attestieren sie aufgrund der „gesteigerten medialen Aufmerksamkeit auf Katastrophen“ und der „Debatten um den anthropogenen Klimawandel“. ${ }^{119}$ Inhaltliche Schwerpunkte des Sammelbandes sind demgemäß Untersuchungen aus stadthistorischer Perspektive im Kontext von Resilienz und Vulnerabilität als Kategorien heutigen Risikobewusstseins, Vergleiche mit der Sturmflut 1953 in den Niederlanden und der Sturmflut in Hamburg 1976, zum Gesellschaftswandel im Mensch-Natur-Technik-Verhältnis, zur Wahrnehmung der Bundeswehr und zur Rolle des Zivilschutzes sowie ein umwelthistorischer Rückblick von Felix Mauch, interpretiert als eine „Nachgeschichte“ der Ereignisse. ${ }^{120}$ Für Mauch ist die Einbeziehung von Analysekonzepten zu Vulnerabilität und Resilienz von großer Relevanz, haben sie doch einen Bezug zur Zukunft, denn „diese richten ihren Blick nicht allein zurück, sondern verknüpfen Erfahrungen des Gestern mit Erwartungen an

114 Vgl. Rieken 2005; vgl. Rieken, Bernd: Angst vor dem Meer. Sturmfluten aus Sicht der volkskundlich-historischen Katastrophenforschung. In: Volkskunde in Rheinland-Pfalz 22, 2007, S. 23-48 (abgekürzt: 2007a).

115 Siehe Paech 2008; siehe Paech, Frauke: Eine Naturkatastrophe gendern?! Erzählungen über die Hamburger Sturmflut von 1962. In: Ariadne. Forum für Frauen- und Geschlechtergeschichte 64, 2013, S. 58-65.

116 Vgl. Heßler, Martina/Kehrt, Christian: Einleitung: Die Hamburger Sturmflut. Betrachtungen aus zeit-, technik- und umwelthistorischer Perspektive. In: Dies. (Hg.): Die Hamburger Sturmflut von 1962. Risikobewusstsein und Katastrophenschutz aus zeit-, technik- und umweltgeschichtlicher Perspektive. Göttingen/Bristol 2014, S. 9-34, S. 10.

117 Ebd., S. 17.

118 Ebd., S. 12.

119 Ebd.

120 Vgl. Mauch, Felix: Die Natur der Katastrophe. Ein umwelthistorischer Rückblick auf die Hamburger Sturmflut. In: Heßler/Kehrt In: Heßler, Martina/Kehrt, Christian (Hg.): Die Hamburger Sturmflut von 1962. Risikobewusstsein und Katastrophenschutz aus zeit-, technik- und umweltgeschichtlicher Perspektive. Göttingen/Bristol 2014, S. 129-148. 
das Morgen. "121 Seine Untersuchungsergebnisse hat Mauch 2015 in einer umfassenden Monografie zu Wahrnehmungs- und Deutungsdiskursen über die Hamburger Sturmflut zwischen 1962 und 2012 ausgeführt. Daher rekurriert er in diesem Grundlagenwerk über die Erinnerungskultur an dieses Extremereignis ebenso auf den 50. Jahrestag sowie auf Museumsausstellungen und heutige Gedenkorte, interpretiert von ihm als materielle Quellen. ${ }^{122}$

Auffällig erscheint indes, dass Zeitzeug_innen als Akteur_innen weder bei Mauch noch bei Heßler und Kehrt Berücksichtigung finden. ${ }^{123}$ Letztgenannte heben allerdings die Potenziale von Zeitzeug_inneninterviews für eine Analyse heutigen Risikobewusstseins hervor. ${ }^{124}$

Untersuchungsergebnisse, die diese Einschätzung gleichwohl stützen, liefert bereits 2009 eine quantitative Studie der HafenCity Universität Hamburg. Darin wird der Bevölkerung des 1962 am stärksten betroffenen Stadtteils, der Elbinsel Wilhelmsburg, ein ausgeprägtes „Risikobewusstsein“ attestiert. ${ }^{125}$ Daran anknüpfend untersucht die Stadtplanerin Mareike Fellmer das bürgerschaftliche Engagement im Umgang mit Sturmflutrisiken. Dafür vergleicht sie Interviewaussagen von behördlichen und zivilgesellschaftlichen Akteuren aus Niedersachsen, Schleswig-Holstein und Hamburg. Ein auch für diese Untersuchung erkenntnisleitendes Ergebnis ist, dass, laut Fellmer, das Erfahrungswissen in Bezug auf die Sturmflut von 1962 heutzutage „eine bedeutende Grundlage für das bürgerschaftliche Engagement“ darstelle. ${ }^{126}$ Ihre Interviewpartner heben den Wert ihrer Erfahrungen in jenen Tagen, „für die Wahrnehmung und das Bewusstsein des Sturmflutrisikos, für die regionale Identität, für Deutungsmuster von Risiko, Schutz und Bedrohung sowie dem heutigen Umgang mit Sturmflutrisiken hervor ". ${ }^{127}$

Demgemäß interpretiert Fellmer Erinnerungskultur als „Ausprägungen von Sicherheits- und Risikokultur“ und folgert „einen Einfluss auf die kulturell geprägte Wahrnehmung und den Umgang mit Sturmflutrisiken" ${ }^{128}$ In ihrem Vergleich der drei Bundesländer wird deutlich, dass Hamburg heutzutage zwar über eine ausgeprägte „Sicherheitskultur“ verfüge, was sich beispielsweise an „dem Vertrauen auf Expertenwissen und technischen Schutzbauwerken "129 zeige. Dieses Vertrauen gehe allerdings auf Kosten einer „Risikokultur“, da somit von der Bevölkerung eine

\footnotetext{
121 Mauch 2014, S. 135, mit Bezug auf Mauelshagen, Franz: Flood Disasters and Political Culture at the North Sea Coast. A Long-term Historical Perspective. In: Historical Social Research 32 (3), 2007, S. 133-144, S. 134.

122 Mauch 2015.

123 Vgl. Eßler, Henrik: (Rezension zu) Mauch, Felix: Erinnerungsfluten. In: Zeitschrift für Geschichtswissenschaft 64 (10), 2016, S. 917-919, S. 919.

124 Vgl. Heßler/Kehrt 2014, S. 17, mit Bezug auf Paech 2008.

125 Vgl. Knieling/Schaerffer/Tressl 2009, S. 68f.

126 Vgl. Fellmer 2014, S. 196.

127 Ebd., S. 196.

128 Vgl. ebd., S. 192.

129 Ebd., S. 196.
} 
eigenverantwortliche Vorsorge nicht als notwendig erachtet werde, mit der Folge, dass diese im Katastrophenfall einer Sturmflut verwundbar sei. Für den Stadtteil Wilhelmsburg kommt Fellmer anhand von Wohnungsbaumaßnahmen im Kontext der Internationalen Bauausstellung (IBA) im Jahr 2013 zu einem ähnlichen Ergebnis. ${ }^{130}$ Als ein Resümee ihrer Untersuchung fordert sie, „dass seitens der staatlichen Küsten- und Katastrophenschutzverwaltungen mehr Möglichkeiten zur zivilgesellschaftlichen Mitgestaltung im Sturmflutschutz geschaffen werden sollten." 131 Dieses Engagement bezieht Fellmer auf ehrenamtliche Tätigkeiten, etwa in Deichverbänden.

Wissenschaftliche Publikationen finden im Gegensatz zur häufig lokal adressierten, ,grauen Literatur' kaum eine breite Leser_innenschaft. Auch massenmediale Erzeugnisse, wie Zeitungsartikel sowie Fernsehfilme und -dokumentationen haben im Gegensatz dazu einen vermutlich größeren Anteil daran, wie das Geschehen heutzutage erinnert und interpretiert wird. Der Fokus ist darin insgesamt aufOpferund Heldengeschichten gerichtet bzw. sind die Figuren dramaturgisch so angelegt, auch weil sich die Darstellung vornehmlich auf die dramatischen Ereignisse während der Nacht beschränkt bzw. darauf zuspitzt. ${ }^{132}$ Durch dieses „Wie von Form, Modus und rhetorischer Ausgestaltung“, hier interpretiert als „mögliche Arten ihrer Darbietung" wirken diese Stories an der spezifischen Form der Tradierung dieser Ereignisse mit. ${ }^{133}$ Dies gilt gleichermaßen für eine Artikelreihe zum 40. Jahrestag 2002 im Hamburger Abendblatt oder in den Harburger Anzeigen und Nachrichten, eine NDR-Reportage, ein ARTE-Doku-Drama und einen RTL-Zweiteiler, der dem Katastrophenfilm-Genre zugeordnet werden kann. ${ }^{134}$

Im Gegensatz zu offiziellen Gedenkreden, musealen Repräsentationen und Denkmälern werden diese Fernsehfilme überregional rezipiert. Und ebenso wie Artefakte des Hochwasserschutzes und Flussmorphologien können sie als Verweise auf gegenwärtige und zukünftige deutschlandweite Risiken in den potenziellen Überschwemmungsgebieten fungieren, wie z. B. 2016 in Baden-Württemberg, aber

130 Vgl. Knieling, Jörg/Fellmer, Mareike: Climate Adaption in Metropolis Hamburg: Paradigm Shift in Urban Planing and Water Management towards ,Living with Water? In: Schmidt-Thomé, Philipp/Klein, Johannes (Hg.): Climate Change Adaptation in Practice: From Strategy Development to Implementation. Chichester u. a. 2013, S. 83-93, S. 83 f.

131 Fellmer 2014, S. 258.

132 Fernsehdokumentationen und Reportagen über umweltkatastrophische Ereignisse, so stellt Kathrin Pöge-Alder am Beispiel des Hochwassers in Sachsen-Anhalt 2013 heraus, betonen „die Themen Not und Rettung“ sowie „die Welle der Hilfsbereitschaft und den Gemeinschaftssinn“ anhand von selbstlos agierenden Einzelpersonen. Vgl. Kawan, Christine Shojaei: Tagungsbericht. 8. Tagung der Kommission für Erzählforschung in der dgv. Gösling, 3.9.-6.9.2014. In: Fabula 55 (1/2), 2014, S. 301-306, S. 305.

133 Vgl. Lucius-Hoene/Deppermann 2004, S. 42.

134 Siehe hierzu Sturmflut. Regie: Lutz Büscher. Fernsehfilm (NDR 1987); Die Nacht der großen Flut. Regie: Raymond Ley. Doku-Drama (NDR/ARTE 2005); Die Sturmflut. Regie: Jorgo Papavassiliou; Drehbuch: Holger Karsten Schmidt. Zweiteiliger Fernsehfilm (RTL 2006). 
auch in der Hansestadt. ${ }^{135}$ Die Hamburger Sturmflut von 1962 wird aufgrund ihrer Bekanntheit heutzutage nach wie vor auch überregional dazu genutzt, eine Gefahrenlage hervorzuheben. Die Süddeutsche Zeitung beispielsweise titelte am 5. Dezember 2013 in ihrem Thema des Tages „Freitag um sechs Uhr wird's kritisch“. Ein Orkan namens Xaver „,bedroht Deutschland“, besonders Hamburg schien dem „Angriff eines echten Rüpels“ ausgesetzt. Und weiter heißt es: „Die Ähnlichkeiten sind frappierend, der Orkan 1962 könnte die Blaupause für Xaver sein“, so wird der Leiter eines privaten Wetterdienstes zitiert. ${ }^{136}$ Bestandteil des ganzseitigen Artikels ist eine mittlerweile ikonische Fotografie der Deutschen Presse-Agentur. Sie zeigt die damalige Rettung von vom Wasser eingeschlossener Bewohner_innen einer Behelfsheimsiedlung.

Dieser fotogestützte Vergleich mit 1962 hat seine Wirkung auf die heutige Leser_innenschaft vermutlich nicht verfehlt. Mehr als fünfzig Jahre liegt dieses Ereignis zurück. Es hat anscheinend weder regional noch überregional wenig von seiner Tragkraft als „Blaupause“, also als Referenz für nachfolgende, als ,Naturkatastrophen' deklarierte Geschehnisse verloren.

Der Begriff ,Naturkatastrophe ${ }^{6}$ muss jedoch einer kritischen Betrachtung unterzogen werden, insbesondere in Hinblick auf seine analytisch weiterführende Relevanz.

\title{
2.2 Der Begriff, Naturkatastrophe' und der Mensch: Zuschreibungsproblematiken
}

\author{
"Contrary to wide spread popular usage, \\ there are no such things as, natural disasters. " 37
}

Aus heutiger Forschungsperspektive fällt es schwer, den Begriff ,Naturkatastrophe eindeutig zu definieren. Einig sind sich die Forscher_innen sozial-, kultur- und geisteswissenschaftlicher Disziplinen darin, dass sich die Deutung dieses Begriffes gewandelt hat: von einer primär religiösen Deutung als Strafe Gottes, die durch naturwissenschaftliche Erklärungen ergänzt sich wandelte hin zu einer Interpretation, die die Hybris des Menschen zunächst mit- und dann hauptverantwortlich macht, und die somit eine Natur, die Opfer des Menschen ist, generiert. ${ }^{138}$ Dies ist jedoch kein alter-

135 Vgl. Mauch 2014, S. 145; vgl. Fischer 2011, S. $165 f$.

136 Vgl. Parnack, Charlotte/Kuhr, Daniela: Angriff eines echten Rüpels. Wie sich Hamburg auf „Xaver" vorbereitet - und mit welchen Problemen auf Schienen und Straßen zu rechnen ist. In: Süddeutsche Zeitung vom 5.12.2013, S. 2.

137 Bankoff, Greg: Comparing Vulnerabilities. Towards Charting a Historical Trajectory of Disasters. In: Historical Social Research 32 (3), 2007, S. 103-114, S. 103, mit Bezug auf Blakie, Piers u. a. (Hg.): At Risk. Natural Hazards, People's Vulnerability and Disasters. London/New York 2004.

138 Vgl. Imhof, Kurt: Katastrophenkommunikation in der Moderne. In: Pfister, Christian/ Summermatter, Stephanie (Hg.): Katastrophen und ihre Bewältigung. Perspektiven und Positionen. Bern/Stuttgart/Wien 2004, S. 145-163, S. 154ff. 
nierender Prozess, verschiedene Denkmuster waren und sind gleichzeitig vorhanden, und sie lassen sich bis ins 16. Jahrhundert zurückverfolgen. ${ }^{139}$ Heutzutage ist allerdings ein deutliches Übergewicht rational-weltlicher Deutungen festzustellen. ${ }^{140}$

Der Hinweis des Schweizer Katastrophenhistorikers Christian Pfister, dass jeder „Naturkatastrophe [...] ein Extremereignis zu Grunde [liegt], das von der Natur ausgeht; aber nicht jedes naturbedingte Extremereignis löst eine Katastrophe aus “ ${ }^{141}$, macht allerdings deutlich, dass der Bezugspunkt, ein Ereignis als ,Naturkatastrophe zu definieren, nicht die naturbedingten Extremereignisse selbst sind, sondern die Auswirkungen, die diese Geschehnisse auf Menschen haben. Die Historiker Dieter Groh, Michael Kempe und Franz Mauelshagen interpretieren demgemäß den Wortbestandteil Natur-als einen Verweis auf die ursachenbedingten Abläufe einer Katastrophe, als eine Zuschreibung, die allerdings den Menschen als (mit-) verursachend ansehen kann. Der Blick auf die Begriffsgeschichte von ,Naturkatastrophe und insbesondere auf lexikalische Definitionen von „Katastrophe“ offenbaren zudem eine Nähe zu Epos und Drama, wenn sie als „Wendung [...] zum Guten oder Bösen, zum Glück oder Unglück“ interpretiert wird. ${ }^{142}$ In dieser Untersuchung, die Erinnerungserzählungen, ist daher ebenfalls nicht die Natur, sondern primär der „auf menschliches Drama“"verweisende Wortbestandteil -katastrophe von erkenntnisleitendem Interesse. ${ }^{143}$

In Weiterführung eines Forschungsdesiderats von Pfister, das Phänomen der ,Naturkatastrophe“ „als ein ganzheitliches Geschehen, das vielerlei Gesichter zeigt “144 zu interpretieren, fokussiert auch die jüngere Forschung vermehrt auf soziale Dimensionen von Extremereignissen, die aufgrund ihrer Komplexität als „hybrid“145 oder als „interdependent“ im Sinne eines „Beziehungsgeflecht[s] zwischen Umwelt und Gesellschaft" verstanden werden. ${ }^{146}$

Die Geografen Carsten Felgentreff und Thomas Glade bezeichnen ,Naturkatastrophen“ als eine „zutiefst menschliche Kategorie“147, denn erst durch die

139 Vgl. Groh/Kempe/Mauelshagen 2003, S. 20f.

140 Vgl. Jakubowski-Tiessen, Manfred/Lehmann, Hartmut: Religion in Katastrophenzeiten: Zur

Einführung. In: Dies. (Hg.): Um Himmels Willen. Religion in Katastrophenzeiten. Göttingen 2003, S. 7-13, S. 11 .

141 Pfister, Christian: Naturkatastrophen und Naturgefahren in geschichtlicher Perspektive. Ein Einstieg. In: Ders. (Hg.): Am Tag danach. Zur Bewältigung von Naturkatastrophen in der Schweiz 1500-2000. Bern/Stuttgart/Wien 2002, S. 11-25, S. 15.

${ }_{142}$ Vgl. Allgemeine deutsche Real=Encyklopädie für die gebildeten Stände. (Conversations= Lexikon) Bd. 6. 8. Auflage. Leipzig 1835, S. 135.

143 Vgl. Groh/Kempe/Mauelshagen 2003, S. 19. Die dem Begriff, Katastrophe‘ innewohnende Unschärfe führt im weiteren Verlauf dieser Untersuchung u. a. zu Fragen der Begriffsverwendung seitens der Befragten. Siehe hierzu Kapitel 4.2.3.

144 Vgl. Pfister 2002, S. 13.

145 Heßler/Kehrt 2014, S. 18.

146 Vgl. Mauch 2014, S. 147.

147 Felgentreff, Carsten/Glade, Thomas: Naturereignisse sind unausweichlich, Katastrophen nicht?! In: Dies. (Hg.): Naturrisiken und Sozialkatastrophen. Heidelberg 2008, S. 443-448, S. 448. 
subjektiven Erfahrungen werde ein Ereignis als katastrophal bewertet. Im Kontext dieser Interpretation entwickelte sich, etwa anhand der Untersuchung von sich in katastrophalen Situationen offenbarender sozialer Ungleichheit, „die Einschätzung des Grades an Verwundbarkeit zum Hauptgegenstand" geistes- und sozialwissenschaftlicher Betrachtung. ${ }^{148}$ In diesem Zusammenhang geraten analytische Zugänge auf Vulnerabilität und Resilienz in den Fokus der Betrachtung und weniger Konzeptionen von ,Natur'. Sie diene heutzutage somit „vielmehr als eine Perspektive auf den Gegenstand" des Katastrophischen. ${ }^{149}$ Auf die volkskundlich-kulturanthropologische Essenz des Begriffes ,Natur' verweist Rolf Wilhelm Brednich, indem er dessen kulturelle Determiniertheit als eine Grundprämisse herausstellt. Er führt aus, dass „die Natur in diesem Sinne als das Ergebnis menschlichen Denkens und Handelns" zu verstehen sei. ${ }^{150}$ Mit ähnlicher Fokussierung hat neben Engels auch unlängst Felix Mauch am Beispiel der Hamburger Sturmflut von 1962 anhand seiner auf zeitgenössischen Quellen basierenden Untersuchungen aufgezeigt, „welche Konzeptionen von Natur eine Gesellschaft im Katastrophenfall entwarf und wie sie sich zu diesen ins Verhältnis setzte. "151 Mauch folgert daraus eine durch damalige Akteur_innen erfolgte Zuschreibung der ,Natur „als Verursacher der Katastrophe.“ Er ordnet diesen Befund analog zu Brednich ein, wenn er resümiert: „Weil Politik, Medien und Öffentlichkeit die Flut als ,Naturkatastrophe' konzeptualisierten, ist sie auch als solche beschreibbar. Denn was sozial konstruiert ist, muss nicht weniger wirklich sein. ${ }^{" 152}$

Somit kann der Begriff, Naturkatastrophe' zwar faktisch als eine Art konsensuales Labeling bezeichnet werden. Als analytische Kategorie für eine, hier interessierende, erfahrungsgeschichtliche Perspektive hingegen liefert er aus den genannten Gründen wenig Anreize.

Diese Entwicklung ist nicht unbeeinflusst von kulturanthropologischen Paradigmen US-amerikanischer Forscher_innen, die auf „the intersections of society, culture, and nature" abzielen. ${ }^{153}$ Diese Schnittstellen begründen insgesamt also primär ein Zusammendenken von Katastrophen und Kultur.

148 Vgl. Walter, François: Katastrophen. Eine Kulturgeschichte vom 16. bis ins 21. Jahrhundert. Stuttgart 2010, S. 19.

149 Vgl. Mauch 2014, S. 132. Hervorhebung im Original.

150 Vgl. Brednich, Rolf Wilhelm: Vorwort. In: Ders./Schneider, Annette/Werner, Ute (Hg.): Natur Kultur. Volkskundliche Perspektiven auf Mensch und Umwelt. 32. Kongreß der Deutschen Gesellschaft für Volkskunde in Halle vom 27.9. bis 1.10.1999. Münster u. a. 2001, S. XI-XII, S. XI.

151 Mauch 2014, S. 147.

152 Ebd. Bereits Gerndt weist auf diese Problematik hin, wenn er das Atomunglück von Tschernobyl als ,Katastrophe“ und als „kulturelle Tatsache" deklarierte, da hier (individuelle und gesellschaftliche) Wertsetzungen immanent gewesen seien. Zudem hebt er anhand dieses Beispiels als „kulturbedingte Vermittlungswege“ die massenmediale Verbreitung hervor. Vgl. Gerndt, Helge: Kulturvermittlung. Modellüberlegungen zur Analyse eines Problemkomplexes am Beispiel des Atomunglücks von Tschernobyl. In: Zeitschrift für Volkskunde 86 (1), 1990, S. 1-13, S. 3.

153 Vgl. Oliver-Smith/Hoffman 2002, S. 17. 


\subsection{Katastrophenforschung: volkskundlich-kulturanthropologische Zugänge}

Dieses „epistemologische Problem “154, dass Ereignisse zwar als ,Naturkatastrophen bezeichnet werden, ohne dass die Bezeichnung jedoch analytische Relevanz besäße, wird in der Volkskunde/Kulturanthropologie etwa auch von den Mitarbeitern des 2015 an der Universität Tübingen abgeschlossenen Drittmittelprojekts mit dem Titel „Lawinen als Bedrohung sozialer Ordnung. Katastrophen im zentralen Alpenraum" vertreten. Jan Hinrichsen, Reinhard Johler und Sandro Ratt gelangen zu der Auffassung, dass der Begriff, Naturkatastrophe' mittlerweile als dekonstruiert und sogar als „historisiert“ gilt. ${ }^{155}$ Anhand zweier Fallbeispiele aus dem 20. Jahrhundert - den Lawinenunglücken in Blons (1954) und Galtür (1999) - werden Verarbeitungsstrategien des jeweiligen Ereignisses durch die Bevölkerung im Kontext einer kollektiven Erinnerungskultur untersucht. ${ }^{156} \mathrm{Im}$ Fokus stehen dabei neben Traditionen vor dem Hintergrund jahrhundertelanger Bedrohungen auch sich wandelnde Deutungen und Bewältigungsstrategien, die vergleichend in den Blick genommen werden. Dabei verbindet Reinhard Johler fachhistorische und heutige Forschungsperspektiven, indem er volkskundlich-kulturwissenschaftliche Katastrophenforschung folgendermaßen definiert:

„[Sie] beschäftigt sich in historischer und gegenwärtiger Perspektive mit der populären Vermittlung von und dem popularen Umgang mit Katastrophen. Deutung und Wahrnehmung - somit die kulturellen Faktoren - von Katastrophen stehen in ihrem Zentrum."157

Diese Begriffsbestimmung im Kontext von „Katastrophenkulturen“158 fußt auf fachgenuinen Untersuchungen der letzten 30 Jahre. Johler nimmt ausdrücklich Bezug auf grundlegende Ausführungen von Helge Gerndt, der 1990 gemeinsam mit Paul Hugger, Ina-Maria Greverus, Utz Jeggle und anderen Fachvertreter_innen einen

\footnotetext{
Mauch 2014, S. 132.

155 In ihrer Begründung führen die Autoren sowohl umfangreiche, primär von der Geschichtswissenschaft durchgeführte Untersuchungen an sowie die Annahme, dass die Forschungsergebnisse zu ,Naturkatastrophen' im Vergleich zu Untersuchungen zu anderen Extremereignissen, wie etwa ,Technikkatastrophen', kaum signifikante Unterschiede aufwiesen. Vgl. Hinrichsen, Jan/Johler, Reinhard/Ratt, Sandro: Katastrophen. Vom kulturellen Umgang mit (außer)alltäglichen Bedrohungen. In: Frie, Ewald/Meier, Mischa (Hg.): Aufruhr - Katastrophe - Konkurrenz - Zerfall. Bedrohte Ordnungen als Thema der Kulturwissenschaften. Tübingen 2014, S. 63, mit Bezug auf Groh/Kempe/Mauelshagen 2003.

156 Vgl. Hinrichsen/Johler/Ratt 2014, S. 61-82.

157 Johler, Reinhard: Die Kultur der Katastrophe und die Materialisierung von Kultur. In: Braun, Karl/Dieterich, Claus-Marco/Treiber, Angela (Hg.): Materialisierung von Kultur. Diskurse Dinge Praktiken. Würzburg 2015, S. 438-443, S. 439. 
Themenband der Zeitschrift für Volkskunde zu ,Katastrophen ' veröffentlichte. ${ }^{159}$ Die Beiträge waren 1988 im Rahmen eines deutsch-französischen Kolloquiums mit dem Titel "Gefahrenzonen/Ethnologie des situations dangereuses" entstanden. Sie befassen sich mit unterschiedlichen Aspekten von Mensch-Gefahr-Beziehungen. Hintergrund dieser Veranstaltung war der GAU im Atomkraftwerk von Tschernobyl im Jahr 1986 gewesen sowie damit einhergehende gesellschaftliche Diskurse über eine „Risikogesellschaft" ${ }^{\prime 160}$ seit Mitte der 1980er Jahre.

Allerdings teile ich nur bedingt die Einschätzung von Johler, dass die Nähe zur „inzwischen tonangebenden historischen Katastrophenforschung“ bei gleichzeitiger Ferne zu „vorbildgebende[n] Untersuchungen“ kulturanthropologischer US-amerikanischer Forscher_innen unter deutschsprachigen Fachvertreter_innen zu einer Vernachlässigung von Positionierungen zu den im Kontext von Katastrophenforschung mittlerweile relevanten „Leitbegriffen“ Resilienz und Vulnerabilität geführt hätten. ${ }^{161}$ Bereits Hugger hatte in dem angesprochenen Band in seinem Aufsatz mit dem Titel „Elemente einer Ethnologie einer Katastrophe in der Schweiz“" zwar diese heutzutage als ,Leitbegriffe' apostrophierten Konzepte nicht genannt, aber bereits explizit die Anfälligkeit einer Gesellschaft bzw. deren Widerstandsfähigkeit und ihr (mangelndes) Vermögen zur Regeneration thematisiert. ${ }^{162}$ In diesem Zusammenhang kommt Hugger in Bezug auf ein durchlebtes Extremereignis und die latente Gefährdung einer Gesellschaft, „die unter dem Damoklesschwert immer wiederkehrender Katastrophen" lebe, anhand seiner Untersuchung in einer Schweizer Region, zu dem Ergebnis, dass die Erfahrungen erstens einen "gemeinschaftsstiftenden Charakter" hatten. ${ }^{163}$ Mehr noch besäßen die Erfahrungen „ein ungeheures soziales Potential, eine Wirkkraft, die als Ferment an der Basis des gesellschaftlichen Lebens stehen“ könne. ${ }^{164}$ Zweitens konstatiert Hugger "spezielle Organisationsformen“165. Diese Kriterien fungieren heutzutage zur Bestimmung von Resilienz und Vulnerabilität einer Gesellschaft, wie im nachfolgenden Kapitel ausgeführt wird. Darüber hinaus stellt Hugger als drittes Kriterium heraus, dass „der moderne Staat dem Bürger weitgehend die Katastrophenvorsorge abgenommen, sie veradministriert

159 Siehe Zeitschrift für Volkskunde 86 (1), 1990. Bereits um 1900 gab es erste fachspezifische Publikationen zum Thema, und auch im Handwörterbuch des deutschen Aberglaubens finden sich für den Kontext relevante Beiträge. Eine Übersicht zur volkskundlichen Katastrophenforschung dieser frühen Jahre siehe Schmidt, Andreas: „Wolken krachen, Berge zittern, und die ganze Erde weint ...". Zur kulturellen Vermittlung von Naturkatastrophen in Deutschland 1755 bis 1855 . Münster u. a. 1999, S. 22ff.

160 Als Schlüsselwerk gilt Beck, Ulrich: Risikogesellschaft. Auf dem Weg in eine andere Moderne. Frankfurt a. M. 1986.

161 Vgl. Johler 2015, S. 440.

162 Vgl. Hugger, Paul: Elemente einer Ethnologie der Katastrophe in der Schweiz. In: Zeitschrift für Volkskunde 86 (1), 1990, S. 25-36.

163 Vgl. ebd., S. 25f. Hervorhebung im Original.

164 Vgl. ebd., S. 25. Hervorhebung im Original.

165 Ebd., S. 25. Hervorhebung durch die Autorin. 
hat. Sie sind in die Hände von Funktionären übergegangen. "166 Dies habe zur Folge, so sein Resümee, dass dort

„[d]as Katastrophenbewußtsein wieder ins Unterbewußte abgetaucht [ist], eine echte Auseinandersetzung mit der existenziellen Gefahr im Sinne eines gemeinsamen sozialen Handelns und eines Überdenkens menschlicher Seinsbedingungen erfolgte nicht. In dieser Hinsicht steht die Schweizer Bevölkerung heute verletzlicher und unreifer da, als die Vorfahren vor zwei, drei Generationen." 167

Huggers analytischer Befund deckt sich mit aktuellen Forschungsergebnissen, etwa wie oben angeführt von Fellmer, zu einer heutigen, mangelnden Risikokultur in Hamburg. ${ }^{168}$ Vergleichbare Forschungsperspektiven eint auch Hinrichsen, Johler und Ratt, wenn sie ihr Interesse an den Relationen zwischen „lokalen Bedrohungstraditionen“ und einer „Bedrohungsvergessenheit" hervorheben, beides Begrifflichkeiten, die bereits bei Hugger aufscheinen. ${ }^{169}$ Darüber hinaus erscheint Huggers Untersuchung auch für heutige Forschungsperspektiven durchaus beachtenswert, auch im Hinblick auf eine auf Strukturen fokussierende, global ausgerichtete Katastrophenforschung nach US-amerikanischem Vorbild, wie sie Johler indes für die fachinterne Betrachtung fordert. Mit Hugger wäre reflektierbar, dass „[j]edes Land seine spezifischen Katastrophen [hat], die typischen Gefährnisse und Krisen. Sie ergeben sich aus unterschiedlichen topographischen, klimatischen, demographischen, wirtschaftlichen und politischen Bedingungen ". ${ }^{170}$

Der von Johler formulierte Befund einer Dominanz historischer Forschungen innerhalb der Volkskunde/Kulturanthropologie ist dennoch nicht von der Hand zu weisen. Die Untersuchung von Andreas Schmidt aus dem Jahr 1999 etwa widmet sich den sprachlichen Codes unterschiedlicher Medien der Jahre 1755 bis 1855, wie etwa Kalendern, Magazinen, Zeitschriften und Kinderliteratur, in denen ,Naturkatastrophen“ thematisiert werden. ${ }^{171}$ Er interpretiert diese Gattungen „als Mittler zwischen dem Alltagsdenken und dem wissenschaftlichen Denken" und untersucht sie demgemäß als medial vermittelte Interpretationsangebote in Bezug „auf ihre Produktion, Funktion und Rezeption" unter der gebräuchlichen Prämisse, dass „Sprache über Kultur transportiert wird und die Sprache selbst Kultur ist. " 172 Schmidts Publikation ist ein Beispiel für den nach wie vor fachlichen Schwerpunkt einer historisch-archivalisch grundierten Perspektive auf ,Naturkatastrophen ' ${ }^{173}$

166 Ebd., S. 34f.

167 Ebd., S. 35.

168 Vgl. Fellmer 2014.

169 Vgl. Hinrichsen/Johler/Ratt 2014, S. $69 f$.

170 Hugger 1990, S. 25.

171 Vgl. Schmidt 1999.

172 Ebd., S. 4.

$173 \mathrm{Zu}$ als ,Technikkatastrophen' deklarierten Ereignissen siehe Helge Gerndt (1990); siehe Frank, Saskia: Zeppelin-Ereignisse. Technikkatastrophen im medialen Prozess. Marburg 2008. 
Die beiden weiteren wichtigen Fachvertreter dieses Forschungsbereiches sind, wie oben bereits erwähnt, Bernd Rieken und Norbert Fischer. Sie eint eine mentalitätsgeschichtliche Grundausrichtung. Während Fischer sozial- und regionalhistorisch fundiert zur norddeutschen Regionalgeschichte des Deichbaus und zu maritimen Gedächtnislandschaften im Kontext von ,Naturkatastrophen ${ }^{6}$ forscht ${ }^{174}$, bezieht sich Rieken als Psychotherapeut und Volkskundler in seinen Untersuchungen vornehmlich auf eine „Kulturgeschichte der Angst“..175 Damit weist er auf die Relevanz psychologischer Fragestellungen auch für die volkskundliche Forschung hin. ${ }^{176}$ Dies ist gerade in Bezug auf die Auswertung historischer Quellen kein unumstrittener Zugang. ${ }^{177}$ Rieken begründet seinen Fokus mit einer Kritik an natur- und sozialwissenschaftlichen Forschungszugängen, die zwar im Kontext eines „integralen Risikomanagement[s] "“ vorgeblich auch die betroffene Bevölkerung als relevanten Faktor ansehen, „ohne jedoch den ,menschlichen Faktor', nämlich Sorgen und Ängste" mit zu berücksichtigen. ${ }^{178}$

Neben der Nordseeküste sind es die Alpen, die als Region im Fokus der volkskundlich-kulturanthropologischen Katastrophenforschung stehen, nicht nur bei Hugger, wie oben bereits ausgeführt wurde. Rieken ist zudem ein Vertreter des fachlichen Schwerpunkts einer Beschäftigung mit als katastrophal deklarierten Ereignissen aus der Perspektive der Erzählforschung. Auch die 8. Tagung der „Kommission für Erzählforschung“ im Jahr 2014 widmete sich dem „Erzählen über Katastrophen“. Darin finden sich Beispiele für eine Bezugnahme auf aktuelle Forschungszugänge US-amerikanischer Provenienz, was die Kritik Johlers relativieren könnte, etwa der Beitrag von Anna Jank. Anhand qualitativer Interviews mit Bewohner_innen auf den Halligen Langeness und Oland über ihre Erfahrungen der 1962er Sturmflut, hebt Jank das „Spannungsverhältnis zwischen Angst und Sicherheit" hervor. Dieses wird von ihr in Anlehnung an Rieken als ein forschungsrelevantes „Grundproblem[s] menschlicher Existenz" interpretiert sowie in Hinblick auf ein „development of coping strategies“, wie hier bereits mit Pfis-

$174 \mathrm{Zu}$ den Untersuchungen von Norbert Fischer siehe ausführlich Kapitel 2.1.

175 Rieken, Bernd: Vom Nutzen volkskundlich-historischer Zugänge für die Katastrophenforschung: New Orleans 2005. In: Hartmann, Andreas/Meyer, Silke/Mohrmann, Ruth-E. (Hg.): Historizität. Vom Umgang mit Geschichte. Hochschultagung „Historizität als Aufgabe und Perspektive“ der Deutschen Gesellschaft für Volkskunde vom 21.-23. September 2006 in Münster. Münster u. a. 2007, S. 149-162, S. 157 (abgekürzt 2007b); vgl. Rieken 2007a.

176 Vgl. Rieken 2005.

177 Marie Luisa Allemeyer bringt es in ihrer Rezension auf den Punkt: „Was, so drängt sich die Frage auf, hat Rieken in die Lage versetzt, derartige Einblicke in die Psyche historischer Akteure zu erlangen?" Allemeyer, Marie Luisa: Nordsee ist Mordsee. Rezension. In: sehepunkte 6 (7/8), 2006: http://www.sehepunkte.de/2006/07/9339.html (aufgerufen am 11.11.2019) sowie die Antwort von Rieken: http://www.sehepunkte.de/2006/09/kommentar/bernd-rieken-ueberrezension-die-geschichte-der-flutkatastrophen-35/ (aufgerufen am 11.11.2019). 
ter formuliert worden ist. ${ }^{179}$ Jank liefert damit ein Beispiel für die Untersuchung von Verhaltensweisen, die als „Bewältigungsstrategie für den erlebten Kontrollverlust“ begriffen werden können. ${ }^{180}$

Nichtsdestotrotz ist die Haltung von Johler gegenüber facheinschlägigen Untersuchungen nachvollziehbar und weiterführend, da es tatsächlich bislang erst wenige gibt, die, anders als der von ihm geleitete Sonderforschungsbereich, sich etwa an dem von Greg Bankoff erarbeiteten Konzept der „Cultures of Disaster“ und seinem Fokus auf „the relationship between a society's vulnerability and the adaption of its culture in terms of local knowledge and coping practices" orientieren und dabei auf Regionen fokussieren, die auch heutzutage einer konkreten bzw. latenten Bedrohung ausgesetzt sind. ${ }^{181}$

In der US-amerikanischen Kulturanthropologie ist seit den 1970/80er Jahren die bereits erwähnte Verwundbarkeit (vulnerability) einer Gesellschaft zum wesentlichen Untersuchungsfeld geworden. ${ }^{182}$ Hierbei wird unterschieden nach sozialen Kategorien, historischer Epoche sowie nach den Darstellungsweisen eines als katastrophal rezipierten Ereignisses. ${ }^{183}$ Diese Kategorien, von Hinrichsen, Johler und Ratt allgemein als Bestandteile einer Katastrophenkultur identifiziert, ${ }^{184}$ stellen Konzepte von Vulnerabilität und Resilienz in den Mittelpunkt auch des volkskundlich-kulturanthropologischen Interesses.

\subsection{Katastrophenkultur zwischen Vulnerabiltät und Resilienz}

Resilienzforschung wurde in den 1950er Jahren zunächst in der Entwicklungspsychologie und seither in der psychologischen Forschung sowie - in einer davon unabhängigen Traditionslinie - in der Naturrisikoforschung und in ökologischen Resilienzdiskursen vertieft. In Letzteren wird die Widerstandskraft vornehmlich in Hinblick auf ihre Bedeutung für den Erhalt eines (Öko-)Systems untersucht. Resi-

\footnotetext{
179 Vgl. Pfister 2011, S. 3.

180 Vgl. Jank, Anna: „Erzählen über Katastrophen“. 8. Tagung der Kommission für Erzählforschung in der Deutschen Gesellschaft für Volkskunde. In: Österreichische Zeitschrift für Volkskunde 117 (3/4), 2014, S. 300-305, S. 302.

181 Vgl. Bankoff, Greg: The Historical Geography of Disaster. ,Vulnerability' and ,Local Knowledge in Western Discourse. In: Ders./Frerks, Georg/Hilhorst, Dorothea (Hg.): Mapping Vulnerability. Disasters, Development and People. London/Sterling 2004, S. 25-36, S. 36.

182 Vgl. Walter 2010, S. 16, mit Bezug auf Wisner, Ben u. a. (Hg.): At Risk. Natural Hazards, People's Vulnerability and Disasters. London/New York 2004, S. XI-XIII. Neben kulturellen Ressourcen als Untersuchungskategorien sind bei Walter „die Mechanismen und die technischen Möglichkeiten, die es erlauben, die Katastrophe zu bekämpfen oder, anders ausgedrückt, die Fähigkeit des Systems, den vorangegangenen Gleichgewichtszustand wiederherzustellen "von analytischem Interesse. Vgl. Walter 2010, S.16.

183 Vgl. ebd., S. 19.

184 Vgl. Hinrichsen/Johler/Ratt 2014, S. 65.
} 
lienz wird dort demgemäß „im Spannungsfeld von Beharrung und Veränderung“ interpretiert, mit Schwerpunkt auf dem „Beharrungsvermögen“ dieses Systems. ${ }^{185}$

Eine weitere Entwicklungslinie stellen seit den 1970er Jahren die oben bereits skizzierten sozialwissenschaftlichen Vulnerabilitätsdiskurse dar. ${ }^{186}$ Von außen hervorgerufene Extremereignisse, wie Umweltkatastrophen oder 9/11, führen die Verwundbarkeit von Sicherheitssystemen vor Augen. Diese werden im Kontext von Resilienz weniger im Zusammenhang von (nicht-)ausreichenden technischen Präventionsmaßnahmen diskutiert, sondern im Kontext normativer Aspekte, wie "class, gender, ethnicity“. ${ }^{187}$

Es lassen sich in Bezug auf gesellschaftliche Resilienz drei Untersuchungsdimensionen unterscheiden: erstens die Widerstandsfähigkeit, als Präventionskomponente verstanden, zweitens die Kapazität, den Ausgangszustand nach einem Extremereignis relativ schnell wiederherzustellen, und drittens sich daraus ergebende Lern- und Anpassungsfähigkeiten. Dieses sind keine statischen Eigenschaften, sondern sie implizieren Prozesshaftigkeit und damit auch Innovationspotenziale. Vulnerabilität und Resilienz sind hierbei als Begriffspaar zu verstehen, sie sind „in einer gemeinsamen Handlungsperspektive verortet und aufeinander bezogen "188 oder, mit dem Soziologen Stefan Kaufmann formuliert, ist Resilienz ein Zugang, um „Sicherheitsprobleme zu reflektieren: Resilienz kommt dort ins Spiel, wo von einer allgemeinen gesellschaftlichen Vulnerabilität die Rede ist. “" ${ }^{189}$ Beide Zugänge lassen sich daher als „prägende[n] Ideen eines umfassenden Sicherheitsdispositivs" bezeichnen. ${ }^{190}$ Gemeinsam ist ihnen, dass sie als Referenz auf den Zustand vor einem Extremereignis rekurrieren. Vulnera-

185 Vgl. Bonß, Wolfgang: Karriere und sozialwissenschaftliche Potenziale des Resilienzbegriffs. In: Endreß, Martin/Maurer, Andrea (Hg.): Resilienz im Sozialen. Wiesbaden 2015, S. 15-31, S. $17 f$. Siehe hierzu Bankoff 2003, S. $5 \mathrm{ff}$.

187 Vgl. Bonß 2015, S. 18. Greg Bankoff, als ein Vertreter dieser Forschungsrichtung, kritisiert, dass „Naturkatastrophen“ innerhalb eines westlichen „cultural discourse“ angesiedelt seien, gegründet auf Verallgemeinerungen und Zuschreibungen zu tropischen Regionen, ihrer vermeintlichen Unterentwicklung und Verwundbarkeit („tropicality, underdevelopment and vulnerability"). Diese Diskurse verunglimpften viele Regionen der Welt als „dangerous - desease-ridden, povertystricken and disaster-prone " und demgemäß werde die Bevölkerung als „inferior, as untutored, incapable, victims" klassifiziert. Die westliche Welt wiederum meine, durch medizinische Versorgung, Investitionen und Präventivsysteme diese Defizite bekämpfen zu müssen. Bankoff stützt sich in dieser Kritik auf seine Untersuchungen auf den Philippinen. Vgl. Bankoff 2003, S. 17. Christmann, Gabriela B./Balgar, Karsten/Mahlkow, Nicole: Zur sozialwissenschaftlichen Konzeption von Vulnerabilität und Resilienz. Konzeptionelle Überlegungen und empirische Betrachtungen am Beispiel von Städten. In: Endreß, Martin/Maurer, Andrea (Hg.): Resilienz des Sozialen. Wiesbaden 2015, S. 123-149, S. 132.

189 Kaufmann, Stefan: Resilienz als ,Boundary Object'. In: Daase, Christopher/Offermann, Philipp/ Rauer, Valentin (Hg.): Sicherheitskultur. Soziale und politische Praktiken der Gefahrenabwehr. Frankfurt a. M./New York 2012, S. 109-131, S. 111.

190 Vgl. Kaufmann, Stefan/Blum, Sabine: Vulnerabilität und Resilienz. Zum Wandern von Ideen in der Umwelt- und Sicherheitsdiskussion. In: Detten, Roderich von/Faber, Fenn/Bemmann, Martin (Hg.): Unberechenbare Umwelt. Wiesbaden 2013, S. 91-120, S. 92. 
bilität und Resilienz werden somit als Fähigkeiten interpretiert, die bereits zuvor bei einem Individuum oder innerhalb einer Gruppe vorhanden gewesen sind.

Resilienz unterscheidet sich damit von Konzepten der Prävention und Vorsorge. Der größte Unterschied besteht darin, dass nicht impliziert wird, Extremereignisse könnten verhindert werden, sondern dieses Konzept zielt darauf ab, „vorab existierende Widerstandspotenziale zu stärken, die dazu führen, dass das entsprechende System in seinen ,Normalzustand' zurückkehren kann - wie immer der auch definiert sein mag. "191 Mit diesem kritischen Nachsatz verweist der Soziologe Wolfgang Bonß darauf, dass dieses Konzept insgesamt in Zusammenhang mit einem auf den oben angesprochenen Vulnerabilitätsdiskursen basierenden, „Paradigmenwechsel im Umgang mit Unsicherheit, [...] hin zu einer Akzeptanz von Unsicherheitspotenzialen jenseits von Prävention und Identifikation von Risikopotenzialen “ stehen müsse. ${ }^{192}$ Bonß prognostiziert in diesem Zusammenhang eine „neue[n] Kultur der Unsicherheit“ und plädiert für „ein prinzipielles Bekenntnis zu Unsicherheit als Basis und Bezugspunkt menschlichen Lebens bei gleichzeitiger Absage an die Idee der Möglichkeit vollständiger Sicherheit." ${ }^{193}$

Damit knüpft er an den heutzutage bestehenden gesellschaftlichen Konsens darüber an, dass Extremereignisse nicht verhindert werden können. Nichtsdestotrotz gehe es darum, möglichst unbeschadet aus diesen hervorzugehen. Implizierte der Begriff der ,Risikogesellschaft' noch einen erfolgreichen Umgang mit zukünftigen Risiken und Gefahren, so trete „an die Stelle des Risikobewusstseins die Anforderung des Umgangs mit Unsicherheit. “194 Demgemäß fokussiert eine Untersuchung von Resilienz auf die Identifikation und Analyse von vorhandenen „Ressourcen, Fähigkeiten, Dispositionen, Strategien etc.“, begriffen als Potenziale, um gegenüber Extremereignissen zu bestehen. ${ }^{195}$ In diesem Zusammenhang können, auch mit dem Geografen Hans-Georg Bohle formuliert, „Wissenssysteme, die historisch akkumuliert und kulturell übermittelt werden“ als eine „Grundvoraussetzung für Resilience“ identifiziert werden. ${ }^{196}$ In seiner Argumentation bezieht er ausdrücklich das Erfahrungswissen der Bevölkerung mit ein, wenn er bilanziert:

191 Bonß 2015, S. 19.

192 Vgl. ebd., S. 16.

193 Bonß, Wolfgang: (Un-)Sicherheit in der Moderne. In: Zoche, Peter/Kaufmann, Stefan/ Haverkamp, Rita (Hg.): Zivile Sicherheit. Gesellschaftliche Dimensionen gegenwärtiger Sicherheitspolitiken. Bielefeld 2010, S. 43-71, S. 65.

194 Gerhold, Lars: Risikobewusstsein. Sicherheit als Konstrukt gesellschaftlicher Wahrnehmung. In: Daase, Christopher/Offermann, Philipp/Rauer, Valentin (Hg.): Sicherheitskultur. Soziale und politische Praktiken der Gefahrenabwehr. Frankfurt a. M./New York 2012, S. 341-356, S. 352.

195 Vgl. Hempel, Leon/Markwart, Thomas: Einleitung. Ein Streit über die Katastrophe. In: Hempel, Leon/Bartels, Marie/Markwart, Thomas (Hg.): Aufbruch ins Unversicherbare. Zum Katastrophendiskurs der Gegenwart. Bielefeld 2013, S. 7-27, S. 7.

196 Bohle, Hans-Georg: Leben mit Risiko - Resilience als neues Paradigma für die Risikowelten von morgen. In: Felgentreff, Carsten/Glade, Thomas (Hg.): Naturrisiken und Sozialkatastrophen. Heidelberg 2008, S. 435-441, S. 436. Hervorhebung im Original. 
„Resiliente Systeme haben es demzufolge verstanden, mit Wandel und Unsicherheit aktiv umzugehen, indem sie aus Krisen gelernt haben, auf das Unerwartete vorbereitet waren und ihr soziales wie ökologisches Gedächtnis gepflegt haben. " 197

Für sein Forschungsgebiet, die humangeografische Gefahren- und Risikoforschung, formuliert Bohle demgemäß das Desiderat, „dass wir besser verstehen lernen, wie Menschen im alltäglichen Umgang mit Risiken diese zu bewältigen suchen “. ${ }^{198}$

Einigkeit in Bezug auf die Ressourcen zur Konstruktion von Resilienz herrscht darüber, dass neben Wissen „um zukünftige Bedrohungen zu erkennen, zu antizipieren und resilienzbildende Maßnahmen zu entwickeln "199 auch materielle - ökonomische und physische - sowie soziale Ressourcen maßgeblich seien, also insgesamt die „Einbindung in ein soziales Netzwerk, hohe persönliche und soziale Kompetenzen, materielle Absicherung etc. “. 200 Auch US-amerikanische Vertreter_innen eines soziologisch ausgerichteten öffentlichen Verwaltungsmanagements betonen die Wichtigkeit für Individuen „to be emotionally connected to their communities, voice their concern, mitigate potential limitations of population diversity, and distribute fairly the roles and responsibilities within the community. ${ }^{\text {"201 }}$

Wir haben es hier mit einer Perspektive auf die Bevölkerung zu tun, die diese nicht als potenzielle Opfer chiffriert, die durch eine administrative Kraft gelenkt bzw. gerettet werden muss, sondern von der zivilgesellschaftliches Engagement erwartet wird. Dass diese Zuschreibungen auch einer kritischen Beurteilung unterliegen sollten, ist nicht von der Hand zu weisen, insbesondere solche, die einer politischen Ökonomie nahestehen, in die neoliberale Strömungen mit eingeschrieben scheinen. ${ }^{202}$ Solcherart Konzepte von Resilienz behindern, so kritisiert die Soziologin und Geschlechterforscherin Sarah Bracke, „skills and capacities of imagining other possible worlds, as well as the agential modalities to pursue those imaginations." ${ }^{203}$ Resilienz fördere somit den Verlust innovativer Vorstellungskraft und, interpre-

197 Ebd., S. 437. Siehe hierzu auch Christmann/Balgar/Mahlkow 2015, S. 134.

198 Bohle 2008, S. 437.

199 Christmann/Balgar/Mahlkow 2015, S. 134.

200 Vgl. Sticher, Brigitta: Das mit dem Unversicherbaren konfrontierte Individuum. Eine psychologische Betrachtung. In: Hempel, Leon/Bartels, Marie/Markwart, Thomas (Hg.): Aufbruch ins Unversicherbare. Zum Katastrophendiskurs der Gegenwart. Bielefeld 2013, S. 307-333, S. 320.

201 Kapucu, Naim/Hawkins, Christopher V./Rivera, Fernando: Disaster Resiliency. Interdisciplinary Perspectives. In: Dies. (Hg.): Disaster Resiliency. Interdisciplinary Perspectives. New York/ London 2013, S. 1-14, S. 9.

202 So weist etwa Sarah Bracke auf die Gefahr hin, das Konzept von Resilienz zu interpretieren „not as a term to describe the ways in which individuals might deal well with the challenges of contemporary society, but as a key to investigate contemporary operations of power and notably to further explore processes of subjectification that belong to the realm of neoliberal governmentality and biopower." Bracke, Sarah: Bouncing Back. Vulnerability and Resistance in Times of Resilience. In: Butler, Judith/Gambetti, Zeynep/Sabsay, Leticia (Hg.): Vulnerability in Resistance. London 2016, S. 52-75, S. 62.

Ebd., S. 64. 
tiert als ein Sicherheitskonzept, das auf Beharrungsvermögen abzielt, verstelle folglich den Blick für gesellschaftliche Entwicklungen. Vulnerabilität werde dementsprechend als Schwäche interpretiert und nicht als grundlegende „ontological characteristic of social relations“. ${ }^{204}$ Diese Sichtweise, Vulnerabilität als ontologische Grundkonstante aufzufassen, kann allerdings aus volkskundlich-kulturanthropologischer Perspektive nicht unumstritten sein.

Für den Theologen und Philosophen Clemens Sedmak ist es ein epistemisches Problem. Er identifiziert drei Sinnsysteme der Resilienzstärkung, die auch für diese Untersuchung weiterführende Erkenntnisse liefern: Erstens die Fähigkeit, eigene Schwäche einzugestehen als Bedingung dafür, Hilfe anzunehmen. Mehr noch ist dies für ihn eine soziale Kompetenz, und der Mensch verfüge demgemäß über einen „sozialen Sinn“. In diesem Zusammenhang steht für Sedmak zweitens ein „Kontrollsinn“, nämlich „Wahl- und Handlungsmöglichkeiten“ und damit insbesondere „[e]in Sinn für Disziplin, Kontrolle und Selbstkontrolle“. Menschen, die sich nicht mehr nur als Opfer sehen, sondern „die sich einer Viktimisierung verweigern“, nehmen bewusst Handlungsspielräume wahr bzw. nutzen diese, um eine Widerstandskraft herauszubilden. ${ }^{205}$ Als die Resilienz stärkenden „Richtungssinn“ macht er drittens aus, dass „Menschen, die ein Ziel vor Augen haben, [...] einen Sinn für Eigenverantwortung und einen Sinn für Wachstum" wahrnehmen. ${ }^{206}$ Sedmak konstatiert für diese drei Sinnsysteme eine identitätsstiftende Funktion, da sie auf soziale Beziehungen, Handlungsoptionen und einen Wertekanon der Menschen rekurrieren. ${ }^{207}$ Für den Aufbau von Resilienz hebt er des Weiteren auch kulturelle Praktiken, wie etwa Rituale, hervor sowie insbesondere die Bedeutung der Familie. Dieses Beziehungsgeflecht mit „Organisationsmuster[n], Kommunikationskultur, Problemlösungsstrategien und positive[n] Orientierungssysteme[n] “ ist - so Sedmak - auch narrativ konstituiert. Wenn beispielsweise eine Familie in der Lage ist, „die eigene Geschichte auch auf Zukunft hin in einer kohärenten Weise zu erzählen“, so könne dies als wichtiger Resilienzfaktor angesehen werden. ${ }^{208} \mathrm{Im}$ Kontext von Resilienz und Vulnerabilität kommt Erinnerungserzählungen somit eine insgesamt große Bedeutung zu.

\footnotetext{
204 Vgl. ebd., S. 69. Sarah Bracke führt aus: „There is, one could argue, an ethical imperative at work here, which holds that one ought to overcome the hazards and shocks of our times and that moral good is to be found in this overcoming." Ebd., S. 62.

205 Vgl. Sedmak, Clemens: Innerlichkeit und Kraft. Studie über epistemische Resilienz. Freiburg i. Br. 2013, S. 30.

206 Vgl. ebd., S. 31.

207 Vgl. ebd., S. 31f.

208 Vgl. ebd., S. 27.
} 


\title{
2.5 Erzählforschung: Reden über katastrophische Erfahrungen
}

\author{
"[These stories] illustrate with astounding clarity the interplay between the event, \\ the personal experience thereof, and the structuring \\ of this experience in a meaningful fashion. ${ }^{209}$
}

Westliche Gesellschaften stellen für die Ausgestaltung von Erfahrungen konkrete Erzählmuster bereit, die es ermöglichen, sich verständlich zu machen und sich untereinander über Erlebnisse auszutauschen. ${ }^{210}$ Auch die in der Gesellschaft verankerte Meistererzählung ordnet sich hier ein. Für Individuen stellt sie sowohl „ordnende“ als auch „normativ-kollektive“ Dimensionen bereit. ${ }^{211}$ Dabei ist von einem spürbaren „Bedürfnis“212 auszugehen, als chaotisch empfundene Erfahrungen in eine kohärente Struktur und in eine inhaltlich kontinuierliche Erfahrungsschilderung zu überführen. Die große Bedeutung dieser strukturgebenden Erzählmuster beschreibt Bendix anhand einer Untersuchung, die sie im direkten Anschluss an das Erdbeben 1989 in der Gegend um San Francisco durchgeführt hat:

„While disaster ultimately implies death of at least a few [...], those who remain thrust themselves into vigorous affirmation of life, and words strung together, eventually finding narrative cohesion, are the first symbolic evidence of our escape from death. "213

Auch die Psychologinnen Helena Topaloglou und Sabine Tschugguel stellen mit Bezug auf das Lawinenunglück von Galtür im Jahr 1999 fest, dass „die kollektive Trauer und Aufarbeitung der Ereignisse [...] durch gelungenes Miteinander-Reden die wesentlichste und hilfreichste Bewältigungsstrategie" gewesen sei. ${ }^{214}$

Dieser Akt des erzählenden sich Mitteilens hat verschiedene Funktionen: zum einen sind dies „solidarisierende Funktionen “215 des Erzählens über ein Ereignis, das von vielen erlebt wurde, dem Bedürfnis folgend, Erfahrungen mit anderen Menschen zu teilen. Dabei stehen nicht Unterschiede von Erfahrungen im Mittelpunkt,

209 Bendix, Regina: Reflections on Earthquake Narratives. In: Western Folklore 49 (1), 1990, S. 331-347, S. 333 .

210 Vgl. Meyer 2014, S. 251. Siehe hierzu auch Lucius-Hoene/Deppermann 2004, S. 248-288.

211 Vgl. Meyer 2014, S. 261.

212 Ebd., S. 256.

213 Bendix 1990, S. 336.

214 Vgl. Topaloglou, Helena M./Tschugguel, Sabine: Lawinenunglück Galtür. Die Frage nach der individuellen Bewältigung aus personenzentrierter Perspektive. In: Rieken, Bernd (Hg.): Wie bewältigt man das Unfassbare? Interdisziplinäre Zugänge am Beispiel der Lawinenkatastrophe von Galtür. Münster 2015, S. 37-61, S. 58.

215 Lehmann, Albrecht: Erzählen eigener Erlebnisse im Alltag. Tatbestände, Situationen, Funktionen. In: Zeitschrift für Volkskunde 74 (2), 1978, S. 198-215, S. 209. Zu den sozial-kommunikativen Funktionen des Erinnerns siehe Kapitel 1.1. 
sondern herausgestellt werden die Gemeinsamkeiten der Erlebnisse. ${ }^{216}$ Eine zentrale Aufgabe, die Erzählungen in diesem Kontext zukommt, ist nach Lehmann, „daß sie Bedürfnisse der einzelnen nach Solidaritätserlebnissen in der Gruppe befriedigen “. ${ }^{217}$ Dieser zumeist unmittelbare Austausch von Erfahrungen zwischen den betroffenen Menschen hat, so Bendix, eine demokratische Anmutung, denn zunächst stehen diese Geschichten gleichwertig nebeneinander und es geht nicht darum, sich durch möglichst spektakuläre Erzählungen bzw. Erzählweisen hervorzuheben. ${ }^{218}$ Im zeitlichen Verlauf wirkt diese Dynamik innerhalb bestimmter Gruppen weiter fort, oder mit Lehmann zusammengefasst: „Zunächst existiert eine Gemeinsamkeit der Erfahrung. Aus ihr ergibt sich im Lebensprozeß eine Gemeinsamkeit des Erinnerns. Schließlich kann daraus eine Gemeinsamkeit des Erzählens werden. “219

Zum anderen besitzen Erzählungen eine auch individualisierende Funktion, begründet dadurch, dass sich jeder „von anderen Menschen vorteilhaft unterscheiden möchte ${ }^{\text {“2220 }}$ bzw. Anerkennung für die eigene Geschichte erlangen und mit ihr irgendwann „erfolgreich" sein möchte. ${ }^{221}$ Außerdem, so führen Topaloglou und Tschugguel aus - interpretiert als Strategie zur Bewältigung eines Extremereignisses - ermöglichen die Geschichten anderer Betroffener „eine gewisse innere Distanzierung [...] und, resonanzbedingt, das Erkennen eigener Gefühle." ${ }^{\text {222 }}$

Die Bedeutung, die insbesondere die Bündigkeit und innere Stimmigkeit einer erzählten Erfahrungsgeschichte in Bezug auf die Verarbeitung von Katastrophenerfahrungen hat, stellt auch die Traumatherapeutin Gisela Perren-Klingler heraus. ${ }^{223}$

216 Vgl. Lehmann, Albrecht: Rechtfertigungsgeschichten. Über die Funktion des Erzählens eigener Erlebnisse im Alltag. In: Fabula 21 (1), 1980, S. 56-69, S. 57.

217 Lehmann 1978, S. 210.

218 Vgl. Bendix 1990, S. 336.

219 Lehmann 1983, S. 25.

220 Lehmann 1978, S. 206.

221 Vgl. Lehmann 1980, S. 57; siehe hierzu auch Welzer, Harald: Das Interview als Artefakt. Zur Kritik an der Zeitzeugenforschung. In: BIOS 13 (1), 2000, S. 51-63, S. 55.

222 Topaloglou/Tschugguel 2015, S. 60.

223 Vgl. Perren-Klingler, Gisela: Katastrophen: Betroffenheit von Einzelnen und Gemeinschaften. Über die Rolle der Narrative beim Umgang mit psychischen Folgen. In: Pfister, Christian/Summermatter, Stephanie (Hg.): Katastrophen und ihre Bewältigung. Perspektiven und Positionen. Bern/Stuttgart/Wien 2004, S. 217-230, S. 217. Mit Perren-Klingler ließen sich die für diese Untersuchung Befragten vermutlich dem sog. Typ-I-Trauma zuordnen. Darunter fasst sie „Menschen mit einem funktionierenden und intakten sozialen Netz, [die] akut auftretende und kurz dauernde einmalige kritische Ereignisse zwar nicht ohne Leiden und Erschütterung [überstehen], doch können sie meist ohne professionelle Hilfe damit fertig werden." Ebd., S. 219f. Zu den psychischen Problemen von Katastrophenopfern siehe auch Ketterer, Werner/Spada, Hans: Der Mensch als Betroffener und Verursacher von Naturkatastrophen. In: Plate, Erich J. (Hg.): Naturkatastrophen und Katastrophenvorbeugung. Bericht des Wissenschaftlichen Beirats der DFG für das Deutsche Komitee für die „International Decade for Natural Disaster Reduction“ (IDNDR). Weinheim u. a. 1993, S. 73-107, S. 80 und S. 91. Darin wird darauf hingewiesen, dass nach Katastrophen ein durchaus breites Spektrum von emotionalen und psychischen Störungen bei den Betroffenen zu beobachten sei, also auch langfristige Beeinträchtigungen, etwa bei Kindern und älteren Menschen. 
Sie hebt mit Blick auf das Sprichwort „geteiltes Leid ist halbes Leid“ die Bedeutung hervor, die ein verbaler Austausch für die Verarbeitung eines Traumas habe. ${ }^{224}$ Auch Rieken betont die Notwendigkeit des Erzählens „als Akt der Psychohygiene“.225

Perren-Klingler verweist in diesem Zusammenhang auf linguistische Aspekte, konkret darauf, dass es für eine gemeisterte Verarbeitung der Erfahrungen spricht, wenn, wie auch im Rahmen dieser Untersuchung deutlich wird, die Sprache kognitiv und präzise ist. ${ }^{226}$ Die Konsistenz und Kohärenz persönlicher Masternarrationen lassen somit Rückschlüsse auf den Grad der Verarbeitung der Erlebnisse zu.

Dass diese Geschichten inhaltlich zunächst sicherlich nicht unverändert bleiben, ist auf den weiteren Verlauf des Extremereignisses zurückzuführen. Durch den direkten Austausch mit Anderen, durch Hörensagen-Geschichten sowie durch Medieninformationen wird zudem die eigene Nahperspektive sowohl räumlich als auch inhaltlich erweitert, wie auch Bendix 1989 im Verlauf der Aufräumarbeiten nach dem Erdbeben in San Fransisco beobachtet: "The stories begin to shift focus; they will dwell more extensively on after-quake observations, criticism of the media, or clean-up activities. Especially strange second-hand narratives are woven into one's own story." 227

Und das persönliche Chaos beginnt sich parallel zu den Aufräumarbeiten ,buchstäblich`zu ordnen:

„Personal narratives are, so I contend, the primary means at an individual's disposal to regain order out of chaos. While fire trucks, bulldozers, construction crews and money allow for the removal of rubble and rebuilding of physical structures, personal narratives accomplish the same work in our heads and hearts." 228

An welchem Zeitpunkt die eigenen existenziellen „Schlüsselerfahrungen“229 zu gestalteten „Schlüsselerzählungen " transformieren, ist nicht exakt zu bestimmen. ${ }^{230}$ Von Bedeutung auch für diese Untersuchung ist, dass sich Schlüsselerzählungen „in ihrer Ausgestaltung [...] verfestigen“, einerseits wenn sie einen besonderen biografischen Bedeutungsgehalt aufweisen und andererseits wenn eine die Erzählerin bzw. den Erzähler bestätigende „kommunikative Wirkung“ erzielt wurde. ${ }^{231}$ Löffler spricht in diesem Zusammenhang von „Erzählwürdigkeit“, also von etwas, das „sich

224 Vgl. Perren-Klingler 2004, S. 223. Weiterführend siehe hierzu auch Alexander, Jeffrey C.:

Towards a Theory of Cultural Trauma. In: Ders. u. a. (Hg.): Cultural Trauma and Collective Identity. Berkeley/London 2004, S. 1-30, S. 2.

225 Vgl. Rieken 2010, S. 189.

226 Vgl. Perren-Klingler 2004, S. 226.

227 Bendix 1990, S. 345.

228 Ebd., S. 333.

229 Lucius-Hoene/Deppermann 2004, S. 119.

230 Vgl. ebd., S. 135

231 Zum Begriff, Schlüsselerzählung siehe ausführlich ebd., S. 33. 
vor dem Publikum bewährt hat, und das mit dem Weitererzählen rechnet, wenn nicht darauf zielt". 232

Die Besonderheiten von Schlüsselerzählungen fassen Lucius-Hoene und Deppermann folgendermaßen zusammen:

„Sprachlich sind sie von ihrer textuellen Umgebung durch eine Erhöhung des erzählerischen Auflösungsgrads, zunehmende Dramatisierung und pointierte Darstellungsweise gekennzeichnet. Oft weisen sie alle Merkmale einer szenischepisodischen Erzählung auf oder sind zur Anekdotenform ausgefeilt." ${ }^{233}$

Von großer Bedeutung in Bezug auf erlebte und erinnerte „Grenzsituationen“ ist zudem, wie Lehmann herausstellt, „die damals herrschende Atmosphäre“. Sie präge „die Qualität der Erinnerung und die Dramatik der Erzählung“ nachhaltig. ${ }^{234}$ Konstituierend hierfür können Sinneseindrücke sein:

„Meistens rekapitulieren diese Geschichten nicht einzelne Sinneswahrnehmungen, sondern bringen die Eindrücke des Gesichts, Geruchs, Gehörs, Geschmacks in der Erinnerung zur Synthese. Synergetische Erinnerungserzählungen dieses Typs wollen dem Leser oder Hörer vermitteln, was damals wahrgenommen wurde und wie es auf den Erlebenden wirkte. "235

Für die Erzählenden kann die Vergegenwärtigung der damaligen Atmosphäre als Einstieg in die Erzählung bzw. als ein insgesamt „wesentlicher Bestandteil“ fungieren. ${ }^{236}$ Auch wenn es sich hierbei ebenfalls nicht um „,objektive“ Eindrücke“ handele, so könne dennoch „von der Art und Weise und von den Inhalten des Erzählens auf die Qualität der Atmosphäre eines erinnerten Erlebnisses“ geschlossen werden.237

In Bezug auf die hier untersuchten Erinnerungserzählungen über die Hamburger Sturmflut von 1962 kann davon ausgegangen werden, wie oben dargelegt wurde, dass die Befragten ihre Erlebnisse bereits mehrfach erzählt haben. Dies führt, in Anlehnung an Ulrike Jureit, zu einer „Verfestigung“ der Erzählungen, also zu

„abgerundete[n], ,flüssig erzählte[n] und in sich geschlossen wirkende[n] Geschichten, die durch häufige Wiederholung immer konstantere Konturen annehmen können. Diese Dynamik kann bewirken, daß sich über viele Jahre Erzählversionen festsetzen, die in gleicher Weise - teilweise in wortwörtlicher

232 Löffler 1999, S. 89, mit Bezug auf Kurt Röttgers: „Eine Geschichte, die nicht weitererzählt werden kann, ist gar keine Geschichte." Röttgers, Kurt: Die Erzählbarkeit des Lebens. In: BIOS 1 (1), 1988, S. 5-17, S. 14; siehe hierzu auch Lucius-Hoene/Deppermann 2004, S. 127f. und S. 146.

233 Lucius-Hoene/Deppermann 2004, S. 135.

234 Vgl. Lehmann 2007, S. 71, mit Bezug auf Böhme, Gernot: Atmosphäre. Essays zur neuen Ästhetik. Frankfurt a. M. 1995.

235 Ebd., S. 72.

236 Vgl. ebd.

237 Vgl. ebd., S. 70. 
Übereinstimmung - rekapituliert werden. Wir haben es also mit Ritualisierungen zu tun. ${ }^{\text {"238 }}$

Diese im Sinne einer Ritualisierung verfestigt-schematischen und chronologischordnenden Erzählungen sind bedeutsam für den Bewältigungsprozess der als existenziell erfahrenen Erlebnisse. ${ }^{239}$ Lucius-Hoene und Deppermann weisen im Kontext der Verarbeitung von Erfahrungen auf die doppelten Zeitperspektiven hin, die sich in Erinnerungserzählungen manifestieren und somit für eine Analyse der Aussagen wichtig sind. Schlüsselerzählungen werden demzufolge zumeist in sogenannter „Erzählzeit" wiedergegeben. ${ }^{240}$ Das darin „erzählte Ich“" orientiert sich zumeist am szenisch-episodischen Erzählen. Mit Hilfe von direkter Rede werden Erlebnisse "re-inszeniert ${ }^{241}$ und ein offener Ausgang der Ereignisse antizipiert. In diese Darstellungsform mischt sich die sachlich-reflektierende „Erzählzeit“ mit dem „erzählende[n] Ich" ${ }^{242}$ Grundlage hierfür sind seither erworbene Wissensbestände, die nun in die individuelle Erzählung einfließen, sie „unentwirrbar“243 machen. Die Tatsache, dass darin die Vergangenheit, also die „erzählte Zeit“, aus heutiger Perspektive geschildert wird, legt nahe, dass ebenfalls heutiges „Wissen, Interpretationsschemata, Argumentationszusammenhänge etc." die Ausgestaltung der Erzählungen maßgeblich prägen. ${ }^{244}$

Diese Ausführungen gelten nicht nur für persönliche Erinnerungserzählungen, auch wissenschaftliche Studien „entfalten eine sprachlich-strukturelle Eigenlogik“ in Bezug auf Krisen und Extremereignisse. ${ }^{245} \mathrm{Zu}$ einem ähnlichen Ergebnis, hier zum ,Mythos der Klimakatastrophe', kommt auch der Soziologe Willy Viehöver. ${ }^{246}$ In seinen Ausführungen über die Bedeutung und Funktion von Sprache bzw. über den „Prozess der Narrativisierung" attestiert er seinen Kolleg_innen, ihre Forschungsergebnisse „im Modus der erzählten Katastrophe“ zu präsentieren. ${ }^{247}$ Für die Darstellung ihrer Untersuchungen montierten sie ,aus den wissenschaftlichen Daten und gesellschaftlichen Hintergrunddeutungen eine kohärente und folgenrei-

238 Jureit, Ulrike: Überlebensdiskurse. Zur Methodik wissenschaftlicher Analyse von Interviews mit Überlebenden der Konzentrations- und Vernichtungslager. Hamburg 1999, S. 88.

239 Vgl. Bendix 1990.

240 Lucius-Hoene/Deppermann 2004, S. 27.

241 Ebd., S. 28. Hervorhebung im Original.

242 Vgl. ebd., S. 116.

243 Lehmann 2007, S. 36.

244 Vgl. Lucius-Hoene/Deppermann 2004, S. 116.

245 Vgl. Meyer, Carla/Patzel-Mattern, Katja/Schenk, Gerrit Jasper: Krisengeschichte(n). „Krise“ als Leitbegriff und Erzählmuster in kulturwissenschaftlicher Perspektive - Eine Einführung. In: Dies. (Hg.): Krisengeschichte(n). „Krise“ als Leitbegriff und Erzählmuster in kulturwissenschaftlicher Perspektive. Stuttgart 2013, S. 9-23, S. 13.

246 Vgl. Viehöver, Willy: Die Klimakatastrophe als ein Mythos der reflexiven Moderne. In: Clausen, Lars/Geenen, Elke M./Macamo, Elisio (Hg.): Entsetzliche soziale Prozesse. Theorie und Empirie der Katastrophen. Münster 2003, S. 247-286.

247 Vgl. ebd., S. 250. Hervorhebung im Original. 
che Geschichte“, so Viehöver. ${ }^{248}$ In seinen Überlegungen nimmt er Bezug auf Hayden White und dessen Argumentation zur „narrative[n] Modellierung“ bzw. zum „Emplotment“ geschichtswissenschaftlicher Texte. ${ }^{249}$

Auch der Film Flut 1962 - Erinnern. Gedenken. Erzählen. - das sei an dieser Stelle bereits als Vorgriff auf das hier praktizierte, analytische Verfahren hervorgehoben - ist das Ergebnis einer Vorgehensweise, in der Erinnerungserzählungen in analytisch begründete Bestandteile zerlegt und in der Phase der Montage erneut zusammengesetzt werden, mit dem Ziel einer, wie White dies nennt, „Explanation by Emplotment“. Diese Art der Encodierung ist demgemäß ein „providing the ,meaning" of a story by identifying the kind of story that has been told“. ${ }^{250}$ Der Gewinn dieser Vorgehensweise liegt darin, die Besonderheiten dieser Erinnerungsgeschichten herauszuarbeiten, oder mit White argumentiert: „Emplotment is the way by which a sequence of events fashioned into a story is gradually revealed to be a story of a particular kind." 251

Dabei geht White mit dem Konzept der „Metahistory“ noch einen Schritt weiter, indem er „die nahe Verwandtschaft zwischen Geschichtsschreibung und literarischen, fiktiven sowie mythischen Textkonventionen" nicht nur anerkennt, sondern fordert, „die sinnstiftenden, konfigurierenden Möglichkeiten der konventionellen Erzählstile für Wirklichkeitsdarstellungen zielbewußt aufzunehmen“, wie die Dokumentarfilmerin Wilma Kiener die Argumentation von White zusammenfasst. ${ }^{252}$

Im Sinne einer „Metahistory“ positioniert sich auch diese Untersuchung, indem sie für die Repräsentation der Forschungsergebnisse auf die erkenntnisgenerierenden Potenziale des Konstruktionscharakters nicht nur der erhobenen Erinnerungserzählungen, sondern auch des Wissensformats Film setzt. ${ }^{253}$

\subsection{Erzählen im Film}

Das Medium Film - dies gilt sowohl für Spiel- als auch für Dokumentarfilme erzählt mit Hilfe einer Sprache, die ebenso wie schriftlicher Text als ein kulturell fundiertes und erprobtes „semantisches Gebilde“254, interpretiert werden kann. Auch wenn es als eigenständig und von der Wortsprache unabhängig angesehen wer-

248 Vgl. ebd., S, 283.

249 Vgl. White, Hayden: Metahistory. Die historische Einbildungskraft im 19. Jahrhundert in

Europa. Frankfurt a. M. 1991, S. 21ff.

250 White, Hayden: Metahistory. The Historical Imagination in nineteenth-century Europe.

Baltimore/London 1975, S. 7.

251 Ebd.

252 Vgl. Kiener, Wilma: Die Kunst des Erzählens. Narrativität in dokumentarischen und ethnographischen Filmen. Konstanz 1999, S. 113f., Hervorhebung im Original.

253 Weiterführend zur Konstruktion des Erzählens siehe Lehmann 2007, S. $50 \mathrm{ff}$.

254 Schmidt-Lauber, Brigitta: Grenzen der Narratologie. Alltagskultur(forschung) jenseits des Erzählens. In: Hengartner, Thomas/Dies. (Hg.): Leben - Erzählen. Beiträge zur Erzähl- und Biographieforschung. Festschrift für Albrecht Lehmann. Berlin/Hamburg 2005, S. 145-162, S. 146. 
den muss, so gilt auch Film als zeichenhaftes und Bedeutung tragendes System. ${ }^{255}$ Seine Lesbarkeit ist für ein Publikum insbesondere dann gewährleistet, wenn sich ein Film einer dramaturgisch motivierten, szenischen Plotstruktur bedient, rekurrierend auf klassische Erzählgenres, wie dem Drama, und wenn er den bekannten und somit dekodierbaren Konventionen der Montage folgt. Dies gilt ebenfalls für kulturwissenschaftliche Filme, wie auch Ballhaus hervorhebt. ${ }^{256}$ Auch sie sind „festgelegten Montageregeln und dramaturgischen Stilen unterworfen", die sich unter anderem darin ausdrücken, dass viele Filme „mit einer ins Thema einführenden Sequenz [beginnen] und [...] mit einem Fazit, einem nachvollziehbaren Ausstieg, einem logischen Schluss" enden. ${ }^{257}$

Film als Wissensformat kann als „dialogische, evokative und polyvokale“258 Form der Kulturrepräsentation die Mehrdimensionalität eines Phänomens sichtbar machen, wie Brigitta Schmidt-Lauber herausstellt. Schwieriger einzulösen erscheint hingegen ihre Folgerung, eine im Film betonte Mehrstimmigkeit könne das Deutungsmonopol des Forschers bzw. der Forscherin aufheben. Als Filmemacherin bin ich diejenige, die, wenn auch nicht sichtbar, die filmische Erzählung formt. Dies geschieht auf einer extradiegetischen Ebene, wohingegen die Protagonist_innen sichtbar und Bestandteil der filmischen intradiegetischen Ebene sind, wenn sie, wie in Flut 1962 - Erinnern. Gedenken. Erzählen., in der Interviewsituation gezeigt werden. ${ }^{259}$ Nichtsdestotrotz treffe ich als Autorin die Entscheidungen bezüglich Auswahl und Gestaltung und folge damit meinem Erkenntnis- und Vermittlungsinteresse. Mein Film bedient sich in Anlehnung an Kiener dem Kommunikationsmodell des „Protagonisten-Erzählers“. Auch wenn ich im Gegensatz zu Kiener, wie oben dargelegt, nicht soweit gehen würde, meine de facto Unsichtbarkeit im Film als erzählextern und der Rolle der sichtbaren Protagonist_innen gegenüber als sich unterordnend zu interpretieren, so, folge ich ihrer Definition, ist

255 Vgl. Kiener 1999, S.17.

256 „Die Dramatisierung der Filmerzählung ist auch bei wissenschaftlich begründeten Dokumentarfilmen ein gängiges Mittel." Ballhaus 2013b, S. 254.

257 Vgl. ebd., S. 255.

258 Schmidt-Lauber, Brigitta: Einleitung zu Panel III: Repräsentationspraxen. Wissens- und Vermittlungsformate kultureller Realitäten. In: Simon, Michael u. a. (Hg.): Bilder. Bücher. Bytes. Zur Medialität des Alltags. 36. Kongress der Deutschen Gesellschaft für Volkskunde in Mainz vom 23. bis 26. September 2007. Münster u. a. 2009, S. 143-147, S. 143. Im Sinne von Schmidt-Lauber hatte bereits Hans-Ulrich Schlumpf eine „Demokratisierung der Leinwände“ gefordert. Vgl. Schlumpf, Hans-Ulrich: Von sprechenden Menschen und Talking Heads. In: Ballhaus, Edmund/Engelbrecht, Beate (Hg.): Der ethnographische Film. Eine Einführung in Methoden und Praxis. Berlin 1995, S.105-119, S. 112. Im Gegensatz zu meiner Vorgehensweise stehen Diskurse zum ethnografischen Film, die sein partizipatorisches Potenzial hervorheben, etwa dadurch, dass die Protagonist_innen sich selbst filmen. Siehe hierzu Walter, Florian: Kollaborative Feld- und Filmforschung. Gleichberechtigte Formen der Kommunikation und transkulturelle Verstehensprozesse. In: Berliner Blätter 67, 2014, S. 56-67.

259 Vgl. Hißnauer, Christian: Fernsehdokumentarismus. Theoretische Näherungen, pragmatische Abgrenzungen, begriffliche Klärungen. Konstanz 2011, S. 295 und S. 299. 
es doch auch mein wesentliches Anliegen, „die innere Fokussierung“ der Interviewten während der Interviewsituation aufzugreifen und in den Mittelpunkt des Films zu stellen. ${ }^{260}$ Da diese Vorgehensweise nur bedingt innerfachlichen Paradigmen entspricht, wonach Visuelle Anthropolog_innen ,am besten ohne, zumindest aber mit wenig Interviews auskommen "261 sollen, werden im Folgenden wesentliche Diskurse zum Einsatz von Interviews im Film vorgestellt und eingeordnet.

\subsubsection{Der ethnografische Film und Interviews}

Die extensive Verwendung von Interviews im Film, zumeist als Talking Heads, also den in halbnaher bis naher Einstellung gefilmten sprechenden Menschen, wurde lange Jahre kritisch beurteilt. Für die Ethnologin Barbara Keifenheim etwa „ist nichts langweiliger als ein Ethnofilm, in dem es nur so von talking heads wimmelt. "262 Ähnlich äußern sich Joachim Wossidlo und Ulrich Roters, wenn sie den „statischen Charakter“ einer Interviewsituation kritisieren, der mit der „visuelle Erzählung“ eines Films breche. Insbesondere bemängeln sie, dass „[d] ie Zeit, die den Sprechenden gegeben wird, um sich selbst darzustellen, [...] auf Kosten der Zeit [geht], die dem Filmemacher für seine Bilder bleiben. " ${ }^{263}$ Hans-Ulrich Schlumpf schließt sich diesem Vorbehalt an. Zudem weist er auf Manipulationsmöglichkeiten bei der Verwendung von Interviews im Off hin, also wenn die Protagonist_innen im Film zwar hörbar, aber nicht sichtbar sind, sowie auf einen inflationären und ,abgegriffenen " Gebrauch „sprechender Menschen“ durch das Fernsehen. ${ }^{264}$ Schlumpf resümiert, „dass uns die intensive Ausbeute sprechender Menschen im Film in eine Sackgasse geführt und von den reichen Möglichkeiten der Filmsprache, die primär eine Bildsprache ist, zu weit weggeführt" habe. ${ }^{265}$

Interviews gesteht Schlumpf allerdings zugleich den Rang eines „möglichen Element[s] unter anderen“ zu und hebt ihre „eigentümliche Kraft“, etwa zur „Aufarbeitung neuerer Geschichte durch Beteiligte“ hervor. ${ }^{266}$ Eine weitere Stärke sieht er darin, dass die audiovisuelle Sichtbarkeit von Protagonist_innen die „Subjektivität der Aussage jeden Moment an den Konnotationen des Bildes nachvollziehbar“

\footnotetext{
260 Zum Erzählertyp des ,Protagonisten-Erzählers' siehe Kiener 1999, S. 254-256.

261 Näser 2014, S. 231. Zu innerfachlichen Diskursen in Bezug auf die Rolle von Interviews im ethnografischen Film siehe ausführlich ebd., S. 231ff.

262 Keifenheim, Barbara: Der Einsatz von Film und Video. In: Beer, Bettina (Hg.): Methoden und Techniken der Feldforschung. Berlin 2003, S. 249-263, S. 259. Hervorhebung im Original.

263 Wossidlo, Joachim/Roters, Ulrich: Vorwort. In: Dies. (Hg.): Interview und Film. Volkskundliche und Ethnologische Ansätze zu Methodik und Analyse. Münster u. a. 2003, S. 7-9, S. 8.

264 Vgl. Schlumpf 1995, S. 113.

265 Vgl. ebd.' S. 115.

266 Vgl. ebd.
} 
mache. ${ }^{267}$ Und er folgert: „Richtig eingesetzt, ermöglicht es (das Element der sprechenden Menschen im Film) auch in Zukunft große Wirkung. " 268

Nichtsdestotrotz exponiert auch Schlumpf Interviewsequenzen im Kontext eines Manipulationsverdachtes, wenn er kritisiert, dass deren Auswahl für den Film „auf der Wahl eines allmächtigen Regisseurs beruht, dessen Methoden für das Publikum schwer nachprüfbar" sei. ${ }^{269}$ Der Kritik kann entgegengehalten werden, dass diese ,Allmacht' außerhalb fachlicher Diskurse unter Dokumentarfilmer_innen mittlerweile weitestgehend als opportun gilt; eine extradiegetische Erzählperspektive wird als grundsätzlich filminhärent angesehen. ${ }^{270}$

Vordergründig reiht sich auch Edmund Ballhaus in die kritischen Beurteilungen von Talking Heads ein, wenn er betont, dass ein wissenschaftlicher Film „wenn er Film bleiben soll, kein verbales Erklärungssystem aufbauen [kann], aus dem sich sein Sinn erschließt. ${ }^{\text {"271 }}$ Gemeinsam ist diesen Haltungen, dass gefilmten Interviews primär nach ihrem - vermeintlich geringen - visuellen Gehalt beurteilt werden. Ballhaus führt zur Stützung seiner Argumentation aus: Film könne „visuell fokussieren und mit der überlegten Abfolge (und Konfrontation) von Bildern, Einstellungen, Sequenzen und Szenen ein Phänomen durchdringen und seinen Sinn offenbaren. "272

Auch er stellt die Aussagekraft gefilmter Interviews tendenziell in Frage, wenn er dabei vernachlässigt, dass auch sie ebenfalls Bilder sind, die erst durch Montagepraktiken und -techniken ihren narrativen „Sinn“ erhalten. Gefilmte Interviews werden somit in erster Linie als abgebildete gesprochene Texte angesehen, die über keinen audiovisuellen Eigenwert verfügen. Torsten Näser interpretiert diese Einstellungen mit Bezug auf die Writing-Culture-Debatten und deren Repräsentationskritiken als innerfachliche „Abgrenzungsbemühungen“ einer Visuellen Anthropologie, die einer „engen Verkopplung von Wissenschaft und Schrift ${ }^{\text {“273 }}$ zu entgehen trachte. Diese Haltung von Fachvertreter_innen erstaunt, insbesondere von Seiten einer Wissenschaftsdisziplin, in deren Fokus der Mensch als handelndes Subjekt steht. Dies schließt ausdrücklich auch die Praktiken des homo narrans mit ein. Paul Henley, ein Vertreter des observatorial cinema, also eines Dokumentarfilmgenres, das sich ausdrücklich nicht auf Interviews stützt, zeigt sich aufgeschlossener, wenn er relativiert:

„However, notwithstanding its limitations, there are certain circumstances in which the interview, be it in some conversational ,disguise' or even in a rel-

\footnotetext{
267 Vgl. ebd., S. 112.

268 Ebd., S. 115.

269 Vgl. ebd., S. 112.

270 Vgl. Kiener 1999, S. 238.

271 Ballhaus 1995a, S. 27.

272 Ballhaus, Edmund: Das Dilemma als Chance. Der kulturwissenschaftliche Film im Prozeß der Feldforschung. In: Lipp, Carola (Hg.): Medien popularer Kultur. Erzählung, Bild und Objekt in der volkskundlichen Forschung. Frankfurt a. M./New York 1995, S. 417-432, S. 430 (abgekürzt: 1995b).

273 Näser 2014, S. 234.
} 
atively formal form, can be an entirely appropriate device for anthropological film-makers, even for those committed to the general precepts of observational cinema." 274

Als Beispiele benennt auch Henley Aktivitäten, die vergangen, nun nicht mehr beobachtbar, jedoch in der Erinnerung abrufbar seien, wie z. B. anhand der örtlichen Umgebung, durch Erinnerungsobjekte oder unter Einbeziehung von zeitgenössischem „supporting“ Material, wie etwa Fotografien. ${ }^{275}$ Des Weiteren führt er innere Vorstellungsbilder und die Erläuterung von Handlungsmotiven an. ${ }^{276}$ Interessant an dieser Haltung ist, dass sie dem innerfachlichen Vorbehalt widerspricht, wonach Film „wohl die Form, nicht aber die Bedeutung festhalten [kann] und dass sich abstrakte Vorstellungen kaum in Bilder übertragen lassen. "277 Diese Einschätzung kritisiert auch Näser, wenn er betont, dass gerade Interviews es ermöglichen, „die thematischen Eingrenzungen, die im Filmdiskurs vor allem durch ein Fokussieren des vorfindbar Sichtbaren markiert sind, aufzubrechen." ${ }^{278}$

Henley stärkt mit seiner Aussage die für diese Untersuchung relevanten Prämissen einer audiovisuellen Argumentation. Mehr noch: seine Position unterstreicht die Potenziale gefilmter Interviews für die volkskundlich-kulturanthropologische Forschung:

„[T]he interviews, broadly defined, is a filmmaking device that anthropological film-makers should be happy to use if and when appropriate. [...] But provided we are aware of their stylistic effects and their epistemological shortcomings, interview techniques can be used in a sensitive manner to elicit a rich variety of oral testimony, ranging from the purely factual to the most intensely emotional. As such, they should form part of every anthropological film-maker's repertoire of skills. “279

Trotz seiner, oben dargelegten, kritischen Haltung gegenüber Interviews nutzt auch Ballhaus sie in dem von Henley skizzierten Sinn: Seine Filme sind sowohl durch Interviews gestaltet als auch Gegenstand analytischer Betrachtung. ${ }^{280}$ Hervorgehoben sei hier insbesondere die Filmreihe zur ostfriesischen Sozial- und Kulturgeschichte. Sie entstand zwischen 2001 und 2009. Die acht Filme haben das Ziel,

274 Henley, Paul: Are you happy? Interviews, ,conversations' and ,talking heads' as methods for gathering oral testimony in ethnographic documentary. In: Wossidlo, Joachim/Roters, Ulrich (Hg.): Interview und Film. Volkskundliche und Ethnologische Ansätze zu Methodik und Analyse. Münster u. a. 2003, S. 51-67, S. 57.

275 Vgl. ebd., S. 61.

276 Vgl. ebd., S. 57.

277 Leimgruber 2010, S. 11.

278 Näser 2014, S. 301.

279 Henley 2003, S. 64.

280 Vgl. Ballhaus, Edmund: Rede und Antwort. Antwort oder Rede? Interviewformen im kulturwissenschaftlichen Film. In: Wossidlo, Joachim/Roters, Ulrich (Hg.): Interview und Film. Volkskundliche und Ethnologische Ansätze zu Methodik und Analyse. Münster u. a. 2003, S. 11-47. 
„die Wechselwirkungen zwischen Landschaft und Lebensformen zu veranschaulichen und die Wandlungsprozesse dieses Kulturraumes im Verlauf des 20. Jahrhunderts zu dokumentieren." ${ }^{281}$ Zudem beabsichtigte Ballhaus, in diesen vom NDR co-finanzierten Produktionen, Stereotypen und Klischees über die Region und ihre Bevölkerung zu zerstreuen. ${ }^{282}$ Daher setzte Ballhaus auf eine bewusste „soziale[n] Kontextualisierung": Drehorte, Bekleidung und eine habituelle Stilisierung, die durch handlungsorientierte Interviews sowie insbesondere durch das von den Protagonist_innen untereinander verwendete „Plattdeutsch“283 erzeugt wird, verweisen auf bestimmte soziale Milieus. Als weitere Stilistik - mit dem Ziel einer „sinnlichen Verstärkung und Veranschaulichung des Erzählinhalts“284 - setzt Ballhaus, neben handlungsorientierten Interviews, häufig auch auf die, wie er dies nennt, „kontextualisierte Erzählung“, dadurch dass Protagonist_innen an für sie bedeutsamen Orten gefilmt werden. Sie fungieren als „Orte[n] der Erinnerung“, um durch die räumliche Vertrautheit einen Erzählfluss zu generieren. ${ }^{285}$ Mit dem Ziel der „emotionale[n] Annäherung" und um die Wirkung der Erzählungen zu verstärken, verschränkt er die gefilmten Interviews darüber hinaus mit (historischen) Fotografien. ${ }^{286}$

Neben diesen, in der Tradition volkskundlich-filmischer Entwicklungen, neuen Darstellungspraktiken benennt Ballhaus das „kollektive Erzählen“ als vornehmliches Stilmittel seiner dialogischen Montage. ${ }^{287}$ Dabei bezieht er sich ausdrücklich auf Eberhard Fechners dokumentarisches Filmschaffen der 1960er bis 1980er Jahre. Dieses soll daher im Folgenden genauer vorgestellt werden, um dessen Relevanz auch für den Film Flut 1962 - Erinnern. Gedenken. Erzählen. zu plausibilisieren.

\subsubsection{Eberhard Fechner}

Der Dokumentarfilmer, Regisseur und Schauspieler Eberhard Fechner gilt als ein Chronist des 20. Jahrhunderts, der immer wieder die Perspektive von ganz normalen Menschen“ einnahm. ${ }^{288}$ Seine Interviewfilme bezeichnete er selbst als „Gesprächsfilme“. ${ }^{289}$ Dieser Terminus verweist auf eine Montagepraxis, die in ihrer Wirkung darauf abzielt, den Eindruck eines Gespräches der Protagonist_innen untereinander

\footnotetext{
281 Ballhaus 2013b, S. 240.

282 Vgl. ebd., S. $239 f$.

283 Ebd., S. 243.

284 Ebd., S. 247.

285 Vgl. ebd., S. $244 f$.

286 Zur Methode der Fotoelicitation siehe Saini, Pierrine/Schärer, Thomas: Erinnerung, Film- und Fotoelicitation. In: Bischoff, Christine/Oehme-Jüngling, Karoline/Leimgruber, Walter (Hg.): Methoden der Kulturanthropologie. Bern 2014, S. 313-330.

287 Vgl. Ballhaus 2013b, S. 257f.

288 Vgl. Netenjakob, Egon: Eberhard Fechner. Lebensläufe dieses Jahrhunderts im Film. Weinheim/ Berlin 1989, S. 12.

289 Vgl. Wilbers, Dörte: „Montierte Erkenntnis“. Überlegungen zur Relevanz der Methoden Eberhard Fechners für den kulturwissenschaftlichen Film. In: Ballhaus, Edmund (Hg.): Kultur-
} 
zu erwecken. Dörte Wilbers spricht hier von „künstliche[n] Dialoge[n]“290, die durch die Montage generiert werden. Fechners Filme sind somit nicht „anhand der Bilder, sondern anhand der Worte“ konzipiert. ${ }^{291}$ Sie verfolgen das Ziel, „durch Kontrast und Widerspruch künstlich Bewusstsein“ herzustellen. ${ }^{292}$ Das Publikum solle angeregt werden, selbst eine Haltung zu entwickeln und zu Einsichten zu gelangen. Auch Flut 1962 - Erinnern. Gedenken. Erzählen. folgt diesem Ansatz. Er expliziert, in Anlehnung an Fechner, auch eine bewusste Autor_innenschaft, die darüber entscheidet, „welche Person an welcher Stelle mit welcher Aussage zitiert wird. “293

Im Gegensatz zu der von Ballhaus postulierten Vorgehensweise ${ }^{294}$, vor Beginn der Dreharbeiten über das Sample der Interviewten bereits entschieden zu haben, wurde darüber hinaus auch hier nach dem Prinzip der Offenheit verfahren. Dies widerspricht dem Ansatz des sogenannten Drehens auf Schnitt, einer auch von Ballhaus vertretenen, vermeintlichen „Tatsache, dass der fertige Film bereits im Feld hergestellt“ werde. ${ }^{295}$ Ein solches Vorgehen, so führt Näser allerdings kritisch aus, verlege „die Gestaltungskraft, die Entscheidungsgewalt des Filmemachers bzw. der Filmemacherin, über die er/sie in der Phase der Montage verfügt, ins Feld vor. [...] Gleichzeitig wird das Tun in der Schnittanlage marginalisiert. "296

Bei Fechner dienen die Dreharbeiten hingegen primär der Interviewerhebung und der Materialsammlung. Dieses Vorgehen ist insbesondere im Filmgenre, Interviewdokumentarismus' gebräuchlich, zu dem auch die Gesprächsfilme von Fechner gezählt werden. ${ }^{297}$ Analog dazu erfolgt auch die Montage der Interviewpassagen für den Film Flut 1962 - Erinnern. Gedenken. Erzählen. nicht nach dem ursprünglichen Interviewverlauf, sondern nach inhaltlichen und ordnenden Kriterien in Hinblick auf die filmische Aussageabsicht. ${ }^{298}$ Übersetzt in eine kulturanthropologisch geleitete Vorgehensweise bezeichnet dies ein Montageverfahren, das, basierend auf der

wissenschaft, Film und Öffentlichkeit. Münster u. a. 2001, S. 275-289, S. 275. Zur Problematik dieser Genrebezeichnung siehe Hißnauer 2011, S. 297.

Wilbers 2001, S. 275, mit Bezug auf Egon Netenjakob, der auf Fechners berufliche Herkunft hinweist, wenn er argumentiert: „Und nur ein Theatermann konnte überhaupt auf die Idee kommen, gegen die cineastische Regel statt vom Bild vom Wort aus zu gehen und künstliche Dialoge zu montieren." Netenjakob 1989, S. 104.

291 Vgl. Wilbers 2001, S. $280 f$.

292 Vgl. Netenjakob 1989, S. 226.

293 Ebd., S. 16.

294 Vgl. Ballhaus 2013b, S. 237.

295 Vgl. Ballhaus 1995a, S. 25.

296 Näser, Torsten: Die Rede vom Stiefkind. Sprachliche Performativität des ethnografischen Films. In: kulturen 2, 2016, S. 37-54, S. 44. „Die Idee dahinter: Die Einstellungen sollen so gedreht werden, dass ihnen im Feld ein im Hinblick auf die Montage handlungsanregendes Potenzial eingeschrieben wird, das im Schnittraum lediglich abzurufen ist“. Ebd.

297 Ausführlich zum Thema ,Interviewdokumentarismus' siehe Hißnauer 2011, S. 287ff.

298 Siehe hierzu ausführlich Kapitel 3.2.4. 
Interviewauswertung und -analyse, dem wissenschaftlichen Erkenntnisinteresse und seiner Vermittlungsabsichten unterworfen ist. ${ }^{299}$

Bevor im Folgenden die methodischen Besonderheiten in der Erhebung, Auswertung und Montage gefilmter Interviews plausibilisiert werden, ist es zunächst notwendig, grundsätzliche methodologische Paradigmen zu diskutieren, die ebenso für nicht gefilmte, also mit Audio-Aufzeichnungsgeräten aufgenommene Interviews, Gültigkeit haben, also die Erhebungsphase der Interviews in ihrer Gesamtheit betreffen.

299 Vgl. Hißnauer 2011, S. 296. 


\section{Interviewerhebung und Auswertung}

Diese Untersuchung ist dem „methodologischen Postulat qualitativer Forschung“ und somit sowohl dem „Prinzip der Kommunikation“, als auch dem „Prinzip der Offenheit" verpflichtet. ${ }^{300}$ Gemeint ist hier, dass das methodische Vorgehen davon bestimmt ist, wie Schmidt-Lauber beschreibt, dass

„sich während der Forschung neue Fragen und Perspektivenwechsel ergeben und die Planung, Materialerhebung, Auswertung und Analyse nur idealtypisch einander folgende Schritte, realiter indes parallel verlaufende Bestandteile eines Prozesses sind. “" ${ }^{\text {301 }}$

Interviews zu filmen dient auch hier zunächst der Materialerhebung, also im Sinne MacDougalls einem „extension of the eye“. Nach ihrer Auswertung und Analyse im weiteren Forschungsverlauf fungieren ausgewählte Interviewaussagen dann, überführt in einen dramaturgisch gestalteten Film mit Aussage- und Vermittlungsabsichten, erkenntnisleitend als „extension of the mind“. .02

300 Schmidt-Lauber, Brigitta: Das qualitative Interview oder: Die Kunst des Redenlassens. In: Göttsch, Silke/Lehmann, Albrecht (Hg.): Methoden der Volkskunde. Positionen, Quellen, Arbeitsweisen der Europäischen Ethnologie. Berlin 2001, S. 165-186, S. 171. Hervorhebungen im Original. Ebd.

302 Vgl. MacDougall, David: Visual Anthropology and the Ways of Knowing. In: Ders.: Transcultural Cinema. Princeton 1998, S. 61-92. 


\subsection{Interviews als kommunikative Praxis}

Interviews ohne Kamera konstituieren sich ebenso wie gefilmte Interviews durch einen primär verbalen Austausch zwischen Interviewer_in und Interviewpartner_in. Hierbei handelt es sich, ob mit Kamera oder ohne, um keine alltägliche Situation: wenn auch auf Freiwilligkeit basierend, sitzen sich zwei in der Regel einander unbekannte Menschen gegenüber; der bzw. die Forscher_in fragt und der bzw. die Zeitzeug_in antwortet. Daher kann diese kommunikative Praxis auch als soziale Handlung begriffen werden. Die Tatsache, dass „ein Interview [...] kein alltägliches, selbstverständliches Gespräch “303 ist, sollte trotz der heutigen Omnipräsenz von Interviews, etwa in Fernsehdokumentationen, nicht außer Acht gelassen werden. Nichtsdestotrotz sind, so führt Schmidt-Lauber aus, „,[d]ie Regeln der Gattung ,Interview' [...] den Gesprächspartnern vertraut". 304

Diese unterschiedlichen Rollen implizieren unterschiedliche Erwartungen und Absichten, Vorerfahrungen und Wissensbestände, und sie evozieren Performanzen: Interviewsituationen sind eine "fragende, zuhörende und antwortende Annäherung“" innerhalb bestimmter Rollen(-zuschreibungen) „die zu gestalten den Interaktionspartnern in einer ganz spezifischen Situation obliegt. “305

Die Reziprozität in der Interviewsituation ist gleichwohl nicht immer eindeutig. ${ }^{306}$ Von Seiten der Interviewenden bestehen ein Forschungsinteresse und der Wunsch, sich als Wissenschaftler_in zu präsentieren. Ihr bzw. ihm gegenüber sitzt der bzw. die Interviewpartner_in, ebenfalls eine bestimmte Absicht verfolgend, nämlich „den thematischen Sachverhalt, die eigene Person oder ein bestimmtes Ereignis auf spezifische Weise zu präsentieren." 307 Zeitzeug_innen gelten als Expert_innen der eigenen Geschichte ${ }^{308}$ und wie Schmidt-Lauber postuliert, sie sollten auch „als solche[r] angesprochen“ werden. ${ }^{309}$ Löffler hebt in diesem Zusammenhang die „in Sozialisationsprozessen erworbene kommunikative und narrative Kompetenz des Befragten" hervor. Unter Verweis auf den von Gabriele Michel verwendeten Begriff der „Erzählkompetenz“ stellt sie die Fähigkeiten der Befragten heraus, „zwischen den unmittelbar und intersubjektiv gesetzten Maßstäben und den mehrfach vermittelten Standards abzuwägen und das biografische Erzählen im Befragungsgespräch zu organisieren.“ Damit einher gehen „Relevanzsetzung[en]“, innerhalb derer die Geschichten erzählt werden. ${ }^{310}$

303 Schmidt-Lauber 2001, S. 177.

304 Schmidt-Lauber 2005, S. 149.

305 Schmidt-Lauber 2001, S. 172.

306 Vgl. ebd., S. 178.

307 Sutter 2013, S. 122.

308 Vgl. Löffler 1999, S. 89.

309 Vgl. Schmidt-Lauber 2001, S. 177.

310 Löffler 1999, S. 90, mit Bezug auf Michel, Gabriele: Biographisches Erzählen - zwischen individuellem Erlebnis und kollektiver Geschichtentradition. Untersuchung typischer Erzählfiguren, ihrer sprachlichen Form und ihrer interaktiven und identitätskonstituierenden Funktion in Geschichten und Lebensgeschichten. Tübingen 1985, S. 53. 
Ein Interview ist demgemäß keine „einsame Handlung des Befragten“, sondern „Produkt der situativen und personalen Bedingungen der Gesprächssituation“, wie auch Welzer hervorhebt. ${ }^{311}$ Eine angenehme Gesprächsatmosphäre wäre somit eine Interaktion, in der beide Seiten sich unbefangen fühlen, danach agieren zu können, wie sie „gesehen werden möchten und sich selbst sehen. “312 Diese Selbstpositionierungen, so Lucius-Hoene und Deppermann, haben „nicht nur beschreibenden Charakter. Mit ihr wird ein bestimmter Selbstentwurf [...] mit selbstvergewissernder Wirkung erhoben: So bin ich, ich möchte mich als diese Person verstehen und verstanden werden. "313

Solcherart interaktive Begegnungen sollten durch die Forschenden reflektiert, etwaige Störungen analysiert und insbesondere das Ideal einer interessierten und aufgeschlossenen, selbstkritischen und insgesamt philanthropischen Grundhaltung beherzigt werden, die das gegenüber als Expert_in der eigenen Geschichte wahrnimmt und anerkennt. Schröder berichtet aus seiner Erfahrung:

„In den meisten Fällen wird ein Interviewer, der Erzählschilderungen ,hervorlocken' will, sowohl ein aufmerksamer, sich mit eigenen Redebeiträgen zurückhaltender Zuhörer als auch ein , anregender', auf den Erzählgegenstand neugierig eingehender Frager sein. " 314

Löffler beschreibt die Interviewsituation demgemäß als eine „Verabredung konzentrierter Aufmerksamkeit." 315

Diese Besonderheiten der Interaktionen während der Interviewsituation gelten sowohl für nichtgefilmte als auch für gefilmte Interviews. Im Folgenden wird, der Chronologie der hier durchgeführten Interviewerhebungsphasen entsprechend, die Vorgehensweise zur Ermittlung von Interviewpartner_innen näher ausgeführt.

\subsubsection{Ermittlung von Interviewpartner_innen}

Die Recherche nach Interviewparter_innen war zunächst beeinflusst von sogenannten key persons, also von Informant_innen, die bereits in Kontakt zum Interviewfeld standen bzw. stehen. ${ }^{316}$ Im Jahr 2003 begann ich meine Recherchen und fand umgehend Unterstützung durch die Leiterin der Geschichtswerkstatt Wilhelmsburg \& Hafen, Margret Markert. Wilhelmsburg war 1962 der am stärksten von den Auswirkungen der Sturmflut betroffene Stadtteil gewesen. Durch die in der Gesellschaft verankerte Meistererzählung gilt er heutzutage als lokales Synonym für das Ereig-

\footnotetext{
311 Welzer 2000, S. 53.

312 Schmidt-Lauber 2001, S. 168.

313 Lucius-Hoene/Deppermann 2004, S. 68.

314 Schröder 1992, S. 15.

315 Löffler 1999, S. 87.

316 Vgl. Lindner, Rolf: Ohne Gewähr. Zur Kulturanalyse des Informanten. In: Jeggle, Utz (Hg.): Feldforschung. Qualitative Methoden in der Kulturanalyse. Tübingen 1984, S. 59-71, S. 59ff.
} 
nis. ${ }^{317}$ Frau Markert stellte mir archivierte Zeitungsartikel und Fotografien zur Verfügung und vermittelte mir erste Zeitzeug_innenkontakte. ${ }^{318}$

Per Brief stellte ich mein Anliegen vor. Die ersten Interviews, die mit Audiokassetten aufgezeichnet wurden, führte ich mit den sowohl in der Geschichtswerkstatt als auch im Heimatverein Wilhelmsburg engagierten Mitgliedern Ursula Falke und Reimar Röttmer zusammen mit dessen Ehefrau Renate Röttmer. Ursula Falke hatte ihre Erfahrungen während der Sturmflut von 1962 bereits in einem Fernsehinterview und in einer Publikation thematisiert. ${ }^{319}$ Im Anschluss an die Interviews vermittelten mir diese ersten Befragten weitere, ihnen persönlich bekannte Gesprächspartner_innen, etwa Familienangehörige oder (ehemalige) Nachbar_innen. Hierbei handelte es sich zumeist um Zeitzeug_innen, die ihre Erlebnisse bisher noch nicht außerhalb ihres jeweiligen persönlichen Umfeldes geschildert hatten.

$\mathrm{Da}$ es nicht nur mein Ziel war, unterschiedliche Jahrgänge sowie paritätisch Frauen und Männer, sondern Betroffene möglichst aus allen damals überfluteten Hamburger Stadtteilen zu interviewen, kontaktierte ich zeitgleich einen Redakteur der Harburger Anzeigen und Nachrichten (HAN), einer Lokalzeitung für den Süderelberaum. ${ }^{320}$ Der Journalist hatte 2002 zum 40. Jahrestag eine Artikelreihe mit Erlebnisschilderungen veröffentlicht. Er vermittelte mir umfangreiche Kontakte zu von ihm interviewten Zeitzeug_innen. Diese waren in der Darstellung ihrer Geschichte ebenfalls nicht unerfahren, und auch sie wirkten als key persons und vermittelten mir weitere Kontakte aus ihrem persönlichen Umfeld.

Die befragten, damals bereits erwachsenen Zeitzeug_innen lebten 1962 aufgrund des Wohnungsmangels und gesellschaftlicher Konventionen zumeist noch bei ihren Eltern. Ehepaare wohnten häufig zusammen mit ihren Eltern bzw. Schwiegereltern in einem Mehrfamilienhaus. Alle gehörten einem ähnlichen sozialen Milieu an. Die meisten interviewten Männer absolvierten damals eine Ausbildung oder arbeiteten als Facharbeiter, zumeist in den hafennahen Industriebetrieben, während die Frauen halbtags häufig im Büro, im Einzelhandel oder „wegen der Kinder“ als Hausfrau tätig waren. Nahezu alle Befragten, die zur Zeit der Sturmflut noch unverheiratet gewesen waren, gründeten in der Folgezeit eine Familie. Zum Zeitpunkt der Interviewerhebungen waren nur noch wenige von ihnen berufstätig. Die Altersspanne der Zeitzeug_innen lag zum Zeitpunkt der Interviewerhebung zwischen 51 und 92 Jahren. ${ }^{321}$

317 Informationen zu den damals überfluteten Stadtteilen siehe Kapitel 2.1.

318 Zur Problematik der Vorauswahl von Informant_innen siehe Lindner 1984, S. 60.

319 Siehe hierzu Falke, Ulla: „Bis zur großen Sturmflut war mir nicht bewusst, dass ich auf einer Insel lebe." In: Geschichtswerkstatt Wilhelmsburg \& Hafen (Hg.): Wilhelmines Gedächtnis. Geschichte weiblich in fünf exemplarischen Lebensläufen. Hamburg 2002, S. 17-39.

320 Die Produktion der Tageszeitung Harburger Anzeigen und Nachrichten wurde im Jahr 2013 eingestellt.

321 Mittlerweile sind einige der von mit interviewten Frauen und Männer verstorben. Eine detaillierte Auflistung mit biografischen Informationen zu den Interviewpartner_innen befindet sich im Anhang. 
Das hier gewählte, nach dem Schneeballprinzip geleitete, Verfahren führte schließlich zu einer Samplebildung, in der „ein spezifisches Netzwerk zutage“ tritt, das auf meinen persönlichen Entscheidungen beruht und somit, wie Schmidt-Lauber kritisch anmerkt, „der Reflexion und Interpretation bedarf. “322 Es besteht aus Zeitzeug_innen, die bis auf wenige Ausnahmen entweder in ihrem damaligen Stadtteil wohnhaft geblieben sind oder dorthin beständig enge Kontakte pflegen. Keine_r der Befragten hatte damals innerhalb des persönlichen Umfeldes einen Todesfall zu beklagen. ${ }^{323}$ Diese Umstände haben sicherlich die Bereitschaft begünstigt, in einem Interview über die damaligen Erlebnisse zu erzählen.

\subsubsection{Interviews erheben}

Zwischen September 2003 und November 2004 führte ich 22 Audio- und 31 Video-Interviews, selten als Kleingruppeninterviews, etwa mit Mutter und Tochter, mit Nachbarn oder mit Ehepaaren. Die Erhebungsphase war bereits abgeschlossen, als im Dezember 2004 ein Tsunami im Indischen Ozean Hunderttausende von Toten forderte. Ich gehe davon aus, dass dieses Extremereignis ansonsten Bestandteil der Interviews gewesen wäre. ${ }^{324}$

Die Interviews erfolgten fast ausschließlich bei den Zeitzeug_innen zu Hause, davon ausgehend, dass dies ein für sie vertrauter Ort ist, eine „Schutzzone“325, an dem sich die Befragten wohlfühlen würden. ${ }^{326}$ Das Wohnumfeld diente somit zur Unterstützung einer offenen Gesprächsatmosphäre ${ }^{327}$, es ermöglichte mir jedoch auch Eindrücke zu erhalten, etwa zu den sozio-ökonomischen Lebensumständen.

$\mathrm{Zu}$ dem Interviewtermin hatten die Gesprächspartner_innen in Eigeninitiative häufig Fotografien, Zeitungsartikel oder Bücher aus ihren privaten Sammlungsbeständen bereitgelegt. Jureit spricht in diesem Zusammenhang von einem „Erfolgsdruck“, unter dem die Interviewten stehen, und davon, dass dieser durchaus Einfluss auf die Interviewsituation haben könne: Um das „von ihnen Mitgeteilte als ,wahr und ,richtig' auszuweisen“, also um ihre Glaubwürdigkeit als Zeitzeug_innen zu untermauern, hielten Interviewte häufig historische Materialien bereit. Jureit argumentiert: „Die erzählte Geschichte erfährt durch diese Objekte eine Illustrierung, die aber in erster

322 Schmidt-Lauber 2001, S. 172.

323 Martha Wolfenstein spricht in diesem Zusammenhang von einer Klassifizierung als „,remote miss" cases“. Wolfenstein, Martha: Disaster. A Psychological Essay. London 1957, o. S., zit. nach Bendix 1990, S. 344.

324 In nur wenigen Interviews wurde auf das Elbehochwasser im Jahr 2002 Bezug genommen. Andere ,Naturkatastrophen' wurden nicht als Vergleich herangezogen.

325 Löffler 1999, S. 88.

326 Almut Leh fordert in diesem Zusammenhang, die Befragten sollten ein „Heimrecht“ zugesprochen bekommen. Vgl. Leh, Almut: Forschungsethische Probleme in der Zeitzeugenforschung. In: BIOS 13 (1), 2000, S. 64-76, S. 65.

327 Vgl. Schmidt-Lauber 2001, S. 178. 
Linie deren Richtigkeit unterstreichen soll. “" ${ }^{228}$ Inwiefern diese Medienerzeugnisse den Interviewpartner_innen bereits als Vorbereitung auf das Interview bzw. als eine Art ,Auffrischungshilfe für ihre Erinnerungen diente, wurde von mir nicht ermittelt, dies kann jedoch vermutet werden. Während der Interviews nahmen die Befragten allerdings keinen direkten Bezug auf das Material.

Die von mir geführten themenzentrierten, narrativen Interviews gliederten sich in drei inhaltliche Frageteile: erstens die Erzählaufforderung durch eine offen formulierte Einstiegsfrage nach dem Ablauf der Ereignisse, konkret der Bitte, mir die Ereignisse der Hamburger Sturmflut von 1962 aus ihrer Perspektive zu schildern und zweitens einer Nachfragephase, um ausführlichere Erzählungen zu den zuvor von den Befragten ggf. nur angerissenen Thematiken zu erhalten. In einem dritten Fragekomplex - der Bilanzierungsphase - standen Fragen nach den individuellen Deutungen und Reflexionen sowie zu Bewältigungspraktiken im Vordergrund.

Leitfadenorientierte Interviews ermöglichen sowohl eine Fokussierung auf thematische Schwerpunkte als auch „eine stärkere Strukturierung und Vereinheitlichung der Gespräche", auch wenn - wie hier geschehen - die Fragen flexibel dem inhaltlichen Verlauf der Interviews angepasst werden. ${ }^{329}$ In der Phase der Interviewanalyse bildet der Leitfaden die Grundlage für eine Vergleichbarkeit der Aussagen. ${ }^{330}$

Grundsätzlich vermittelt die Verwendung von Audiorekorder bzw. Filmkamera den Befragten nicht nur den Eindruck einer professionellen Vor- und Nachbereitung der Interviews, vielmehr unterstreichen die Aufzeichnungen die Expert_innenrolle der Zeitzeug_innen, wie etwa Almut Leh herausstellt: „Auf sie ist das Mikrophon ausgerichtet; der Interviewer ist auf die Rolle des Zuhörers verwiesen. Für den Erzähler ist er ein Stichwortgeber [...]. Die eigentliche Zuhörerschaft - dafür steht das Tonband - ist viel größer." "331

Diese Inwertsetzung wird durch das Filmen eines Interviews umso mehr betont. Gerade weil die Aufnahmetechnik während der Interviewsituation nicht gänzlich vergessen wird, bleibt den Befragten bewusst, dass die Äußerungen von großer Relevanz für das Forschungsvorhaben sind.

\subsubsection{Interviewtranskriptionen - ein Problemaufriss}

Im Gegensatz dazu stehen Reaktionen der Interviewpartner_innen auf die Verschriftlichung ihrer Aussagen. Im Forschungsprozess dienen Transkriptionen primär als analytische Hilfsmittel und somit der Erkenntnisgewinnung. Die Besonderheiten dieser ungewöhnlichen Textgattung ließen sich gegenüber denjenigen, darum baten, ihre Äußerungen gegenzulesen, allerdings nur bedingt vermitteln.

328 Jureit 1999, S. 90. Sie führt als ein „weiteres Mittel der Beweisführung“von Seiten der Befragten an, dass diese sich auf andere Personen berufen. Inwiefern die Vermittlung weiterer Gesprächspartner_innen durch die Befragten diesem Zweck diente, wurde hier nicht ermittelt.

330 Vgl. Sutter 2013, S. 124.

331 Leh 2000, S. 67. 
Die Erstellung von Interviewtranskriptionen ist ein Übersetzungs- und damit auch Interpretationsvorgang, dadurch dass verbal getätigte Äußerungen in einen schriftlichen Text überführt werden. ${ }^{332}$ In einem ersten Arbeits- und Reflexionsschritt habe ich die Transkriptionen auf ihre Richtig- und Vollständigkeit hin überprüft. Schmidt-Lauber empfiehlt bei einer Analyse, die auf einer Vielzahl von Interviews basiert, frühzeitig im Untersuchungsverlauf Transkriptionen anzufertigen. Damit werde ermöglicht, „,erste Interpretationen, auffällige Lücken oder offene Fragen in den weiteren Gesprächen berücksichtigen zu können und den Interviewstil kritisch zu überprüfen. “333 Diese sukzessive Vorgehensweise hat sich auch hier, dem Prinzip der Offenheit qualitativer Forschungsmethoden folgend, als erkenntnisleitend erwiesen.

Eine kommunikative Praxis der besonderen Art stellt indes die Weitergabe von Interviewtranskripten an die Interviewten dar, wie oben bereits angedeutet wurde, denn diese hat - im Gegensatz zu den bisherigen Arbeitsschritten - auch zu Missstimmungen und Konflikten geführt. ${ }^{334}$ Transkriptionen, also die ungeglätteten Niederschriften der gesprochenen Worte, sind als Textsorte gemeinhin nicht geläufig; die schwarz auf weiß fixierten Äußerungen waren den Interviewten deutlich unangenehm. Leh hebt bedauernd hervor: „Selten hat der Zeitzeuge Sinn für den Charme seiner abgebrochenen Sätze und abgeschliffenen Wortendungen. Kaum ein Satz, der auch nur annähernd grammatikalischen Grundregeln genügt. "335 Auch die Erfahrungen aus dieser Untersuchung legen die Vermutung nahe, dass „interessanterweise es zumeist vor allem die Sprache [ist], durch die der Zeitzeuge sich bloßgestellt fühlt; selten sind es seine Geschichten“.336

Sollen Auszüge aus Interviewtranskriptionen Eingang in eine Forschungsarbeit finden, so werden sie zumeist zum Zweck einer „besseren“ Rezipierbarkeit geglättet. ${ }^{337}$ Dies gilt nur bedingt für die Interviewaussagen, die im Film Flut 1962 - Erinnern. Gedenken. Erzählen. verwendet werden. Gesprochener Text unterliegt im Film einer anderen Beurteilung als in einer schriftlich fixierten Arbeit, weil Menschen im gefilmten Interview eher zugestanden wird, nicht immer flüssig zu reden. Dies interpretiere ich als einen Mehrwert der filmischen Darstellung von Interviewpassagen, weil so der Handlungscharakter gesprochener Worte unterstrichen werden kann.

\footnotetext{
332 Zur Problematik von Interviewtranskriptionen siehe Schmidt-Lauber 2005, S. 155ff. sowie grundlegend Schröder 1992, S. $79 f f$.

333 Schmidt-Lauber 2001, S. 179.

334 Ein Interviewpartner hatte mir nach Durchsicht die Nutzungsrechte entzogen, eine weitere Transkription durfte nur in der durch den Neffen einer Interviewpartnerin überarbeiteten Version verwendet werden.

335 Leh 2000, S. 70. Leh weist darauf hin, dass diese Reaktionen durchaus ebenfalls interpretationswürdig wären.

336 Ebd., S. 71.

337 Vgl. Sutter 2013, S. 127.
} 


\subsection{Interviews im Wissensformat Film}

Die Entscheidung, Film als Wissensformat, also zur Generierung und als Repräsentationsmedium von Forschungsergebnissen zu nutzen, hat Auswirkungen auf die methodische Vorgehensweise: weniger auf die Auswertung der Interviews als solche, gleichwohl auf für die Interviewerhebungssituation. Die Kameraarbeit folgte hierbei für Flut 1962 - Erinnern. Gedenken. Erzählen. insgesamt einem sich eher zurücknehmenden, naturalistischen Modus, der weder beobachtend, noch evokativ wirken soll. ${ }^{338}$ Die Kamera war auf einem Stativ befestigt, um während des Interviews ruhige Aufnahmen generieren zu können und um eine feste Kameraperspektive zu gewährleisten.

Die Dreharbeiten wurden gemeinsam mit einem Kameramann durchgeführt. Andree Kummerfeld und ich hatten bereits zuvor zwei volkskundlich-kulturanthropologische Filme realisiert, und die gemeinsame fachlich-methodologische Auseinandersetzung, insbesondere zum gefilmten Interview, bildete die Grundlage auch für diesen Forschungsfilm. ${ }^{339}$ Eine Aufteilung der Verantwortlichkeiten hat sich dabei als sinnvoll erwiesen. Eine Fokussierung auf die jeweiligen Tätigkeiten Andree Kummerfeld war zuständig für die Aufnahmetechnik, auch in Bezug auf Licht und Ton, während ich die Interviews führte - hatte den Vorteil, dass keine Kamera die Blickachse zwischen Interviewpartner_in und Interviewerin verstellte. Der Kameramann befand sich in der Position des „versteckten und geschützten“ Zuschauers, er hatte Distanz zum Geschehen, um seine Arbeit konzentriert durchführen zu können. ${ }^{340}$ Dies gilt gerade dann, wenn die filmende Person nicht in die Gesprächssituation miteinbezogen ist. Auch an der Auswahl von Interviewpartner_innen und Drehorten war der Kameramann nicht beteiligt.

\subsubsection{Interviewpartner_innen auswählen}

Die Auswahl der Zeitzeug_innen für ein gefilmtes Interview war nicht vorrangig aufgrund eines ,artistic calibre“ erfolgt ${ }^{341}$, also nicht aufgrund subjektiver Bewertungen unterliegender, darstellerischer Fähigkeiten, wortgewandt und pointenreich erzählen zu können. Während Ballhaus die Notwendigkeit eines „Anforderungsprofil[s]“ für die Auswahl von Interviewpartner_innen im Film hervorhebt - er führt in diesem Zusammenhang sprachliche Eloquenz und sowie eine ,bildfüllende' Ausstrahlung $\mathrm{an}^{342}$ - so war dies für Flut 1962 - Erinnern. Gedenken. Erzäblen. kein wesent-

338 Siehe grundlegend hierzu MacDougall, David: Ein nichtpriviligierter Kamerastil. In: Friedrich, Margarete (Hg.): Die Fremden sehen. München 1984, S. 73-83.

339 Weitere Filmprojekte mit Andree Kummerfeld: „So lange der Michel steht“ - Selbstbild und Alltagserfahrungen in den lebensgeschichtlichen Erzählungen zweier homosexueller Männer (2001) sowie (gemeinsam mit Anne-Katrin Becker) „Je näher ich dran bin, umso weniger verstehe ich davon" - Gespräche über den Tod (1995).

340 Vgl. Ballhaus 1995a, S. 30.

341 Bendix 1990, S. 336.

342 Vgl. Ballhaus 2003, S. 34. 
liches Kriterium. ${ }^{343}$ Primär erfolgte die Auswahl der Interviewpartner_innen anhand der bereits genannten geschlechterparitätischen und lokalen Gesichtspunkte. Der Tatsache, dass Wilhelmsburg der damals am stärksten betroffene Hamburger Stadtteil gewesen ist, entspreche ich dadurch, dass die meisten gefilmten Interviews mit Zeitzeug_innen, die damals dort lebten, geführt wurden. Gleichwohl ist in der späteren Arbeitsphase der Montage die Entscheidung zwischen gleichen Aussageinhalten nicht gänzlich unbeeinflusst von der verbalen Ausdrucksfähigkeit und dem Vermögen der Befragten, ihre Geschichte stringent erzählen zu können. ${ }^{344}$

Eine grundsätzliche Scheu, sich in der Interviewsituation filmen zu lassen, sich somit also der zusätzlichen Anwesenheit von Kamera, einer filmenden Person und ggf. Scheinwerfern auszusetzen, konnte nicht festgestellt werden. Insgesamt gehe ich davon aus, dass dieses Befragungsverfahren aufgrund von Rezeptionserfahrungen mit dem Medium Fernsehen mittlerweile bekannt ist. In die Definition von Löffler einer Interviewsituation als „[e] in szenisches Arrangement mit Tradition“345 möchte ich daher das gefilmte Interview mit einbeziehen sowie mit Ballhaus die Dreharbeiten darüber hinaus im Lichte eines emanzipatorischen Effekts interpretieren: „Viele Gefilmte erleben die filmische Dokumentation ihrer Anschauungen, ihrer Biographie, ihres Lebensumfeldes als Stärkung ihrer Identität. Ihnen gilt das Interesse des Drehteams. Sie sehen sich dokumentiert." ${ }^{436}$

Nichtsdestotrotz ist die Situation der Dreharbeiten in ihrer Gesamtheit als ein potentieller Einflussfaktor auf die ohnehin immer interaktionistische Interviewsituation zu reflektieren. ${ }^{347}$

\subsubsection{Interviews filmen}

Die Auswertung der zuvor nicht gefilmten Audio-Interviews hatte zu der Entscheidung geführt, Flut 1962 - Erinnern. Gedenken. Erzählen. als Interviewfilm im Sinne Fechners anzulegen, also nicht nur exemplarisch einige wenige Protagonist_innen mit ihren exemplarischen Geschichten in den Mittelpunkt des Films zu stellen, sondern die Besonderheit sowohl der Kongruenzen als auch der Vielstimmigkeit hervorzuheben. Diesem Vorhaben liegt im Hinblick auf das filmische Endprodukt eine bestimmte ästhetische Arbeitsweise zugrunde, die bereits während der Dreharbeiten reflektiert und umgesetzt werden muss. Dieses Vorgehen zielt auf zwei filmische

343 Alexander von Plato weist jedoch bei diesem auf Vielstimmigkeit angelegten Verfahren zu Recht auf die Gefahr hin, „Homunkuli“ zu produzieren, wenn sich Wissenschafter_innen ermächtigen, eine Auswahl darüber zu treffen, wessen Aussagen medial erhalten und überliefert werden. Vgl. Plato, Alexander von: Medialität und Erinnerung. Darstellung und „Verwendung" von Zeitzeugen in Ton, Bild und Film. In: BIOS 21 (1), 2008, S. 79-92, S. 89. Zur Problematik einer Auswahl von Interviewpartner_innen vgl. ebd., S. $84 f$.

344 Siehe hierzu ausführlich Kapitel 3.2.3.

345 Löffler 1999, S. 76.

346 Ballhaus 2013b, S. 238.

347 Eine kritische Haltung gegenüber gefilmten Interviews siehe Plato 2008. 
Strategien ab, die miteinander kompatibel sein sollen, nämlich darauf, sowohl die gesellschaftlich vorgeprägten Erzählmuster als auch die Individualität der Protagonist_innen darzustellen. Dabei sollen die Einzelaussagen als überindividuell dekonstruiert werden können, gleichzeitig gilt es, die Protagonist_innen nicht zu egalisieren. Um dies zu erreichen, habe ich mich bei jedem Interview für eine ähnliche Kameraeinstellung entschieden: bei den Interviews unbewegt und halbnah, fokussiert auf den bzw. die jeweilige Protagonist_in. ${ }^{348}$ Erst dann ist im Schnitt gewährleistet, dass die einzelnen Interviewausschnitte „durch eine[n] andere[n] fortgesetzt, ergänzt, bestätigt, erläutert" werden, wie Egon Netenjakob für die Wirkungsweise der Montage bei Fechner herausstellt ${ }^{349}$, und dabei - begünstigt durch die immer wieder identische Einstellungsgröße - zu dem intendierten künstlichen Dialog verschmelzen können.

Um individualisierende Effekte innerhalb dieser bewusst sowohl auf Musterbildung als auch auf Differenz hin konzipierten filmischen Narration zu erzielen, wurden die Interviewten vor unterschiedlichen Bildhintergründen gefilmt. Dies ermöglichte eine Vermittlung ergänzender Informationen und Eindrücke zu der jeweils sprechenden Person. ${ }^{350}$ In der filmischen Rezeption wiederum begünstigt dies eine Unterscheidbarkeit der insgesamt 19 Protagonist_innen bzw. einen Wiedererkennungseffekt. ${ }^{351}$

\subsubsection{Interviews auswerten}

Auch wenn der Mehrwert einer audiovisuellen Repräsentation der Zeitzeug_innen auf der Hand liegt, nicht nur durch die Hörbarkeit der Stimme, ${ }^{352}$ sondern auch durch die Sichtbarkeit der Befragten, also der Kombination aus Gestik, Mimik und Körperhaltung sowie der Kleidung, so sind hier primär die Aussageinhalte, also die verbal getätigten Aussagen, von analytischem Interesse. Somit wird im Rahmen die-

348 Zu den Konventionen gefilmter Interviews: „Wesentlich dafür sind die Position der Protagonisten (sitzend), die Einstellungsgröße (Nah oder Groß), die Perspektive (Normalsicht/Kamera in Augenhöhe) und die Blickrichtung." Hißnauer 2011, S. 298.

349 Netenjakob 1989, S. 103. Ausführlich zur Arbeitsweise von Eberhard Fechner siehe Kapitel 2.6.2.

350 Bei einem identischen Hintergrund, bei Studioaufnahmen z. B. vor einem schwarzen Tuch mit Lichtsetzungen, wie dies häufig in Fernsehproduktionen üblich ist, werden biografische Hintergrundinformationen über Texteinblendungen, sogenannten ,Bauchbinden', vorgenommen.

351 Diese Darstellungsweise geht allerdings auf Kosten einer detaillierten biografischen Kontextualisierung, die, anders als es etwa durch filmische Porträts möglich wäre, hier weitestgehend minimiert ist. Vgl. Schröder, Hans Joachim: Biografisches Erzählen im Umgang mit Technik. In: Seifert, Manfred/Friedreich, Sönke (Hg.): Alltagsleben biografisch erfassen. Zur Konzeption lebensgeschichtlich orientierter Forschung. Dresden 2009, S. 61-68, S. 65.

352 Vgl. Bendix, Regina: Die Stimme: Eine Spurensuche. In: Hengartner, Thomas/Schmidt-Lauber, Brigitta (Hg.): Leben - Erzählen. Beiträge zur Erzähl- und Biographieforschung. Festschrift für Albrecht Lehmann. Berlin/Hamburg 2005, S. 71-95. 
ser Untersuchung der visuellen Ebene innerhalb der gefilmten Interviews ein analytisch „lediglich ergänzende[r] Stellenwert“ zugesprochen. ${ }^{353}$

Die Analyse der Interviews operierte auch hier in einem „Spannungsfeld zwischen intuitiven und systematischen Elementen“, wie Gerrit Herlyn für gängige kulturanthropologische Auswertungspraktiken bilanziert. ${ }^{354}$ Nichtsdestotrotz bildeten „die expliziten Selbstdeutungen“der Interviewpartner_innen den grundlegenden Ansatzpunkt, mit dem Ziel „die Sinnkonstruktion“ der Befragten offenzulegen. 355 Eine Orientierung an den subjektiven Erzählungen bildete die Grundlage, um auf strukturelle und inhaltliche Besonderheiten aufmerksam zu werden und im weiteren Auswertungsverfahren auf diese fokussieren zu können. ${ }^{356} \mathrm{Als}$ wesentliches Analysewerkzeug dienten dabei die Transkriptionen der sowohl per Audiogerät als auch per Video aufgezeichneten Interviews. Nach ihrer Überprüfung auf Vollständigkeit erfolgte eine Unterteilung nach inhaltlichen Schlagwörtern, die in analytische Kategorien überführt wurden. Der von Schmidt-Lauber gewählte Zugang einer „vergleichenden Zusammenschau der Interviews bzw. von Interviewausschnitten aufgrund ausgewählter Kategorien" integriert auch in dieser Untersuchung die geführten Interviews in ihrer Gesamtheit und bündelt sie unter dem Begriff der „selektive[n] Plausibilisierung“, da „zu einzelnen thematischen Bereichen aus unterschiedlichen Interviews Beispiele zitiert“ werden ${ }^{357}$, mit dem Ziel, „Verdichtungen und Verallgemeinerungen " herzustellen. ${ }^{358}$

Ein volkskundlich-kulturanthropologisch argumentierender Film, der Erinnerungserzählungen in seinen Mittelpunkt stellt, muss zudem bei aller Verdichtung mehrerer Aussagen auch der Bedeutung subjektiver Einzelaussagen gerecht werden, zumindest dann, wenn der bzw. die Filmemacher_in ohne Kommentar auskommen will. Somit an das empirische (Roh-)Material gebunden, besteht keine Möglichkeit, Aussagen zu paraphrasieren. Hinzu kommen Rezeptionserwartungen eines Publikums an die Länge eines Dokumentarfilms, die es erforderlich machen, dramaturgisch zuzuspitzen und Inhalte zu priorisieren. Diese Mehrfachanforderungen können auf Kosten einer allumfassenden Darstellung der Interviewaussagen, die für die Untersuchung relevant sind, gehen, wie das Beispiel der Erinnerungserzählungen im Kontext sozial-räumlicher Orientierungen von Mitgliedern der Familie

353 Vgl. Bohnsack, Ralf: Qualitative Bild- und Videointerpretation. Die dokumentarische Methode. 2., durchgesehene und aktualisierte Auflage. Opladen/Farmington Hills 2011, S. 138. Zur Einführung in die Analyse videografierten Datenmaterials siehe Rosenthal, Gabriele/Witte, Nicole: Zur Analyse videographierten Datenmaterials. In: Rosenthal, Gabriele: Interpretative Sozialforschung. Eine Einführung. 5. aktualisierte und ergänzte Auflage. Weinheim/Basel 2015, S. 130149.

354 Herlyn 2013, S. 487.

355 Vgl. Leh 2000, S. 72. Gleichwohl stellt jede Interaktionssituation eine auch performative und kommunikative Praxis dar, die somit nicht als von den Interviewenden unbeeinflusst interpretiert werden kann. Siehe hierzu auch Kapitel 3.1.

356 Vgl. Sutter 2013, S. $128 f$.

357 Vgl. Schmidt-Lauber 2001, S. 181.

358 Vgl. Herlyn 2013, S. 485. 
3 Interviewerhebung und Auswertung

Meyer aus Francop in Kapitel 4.2.2 verdeutlicht. Es fand trotz seiner unzweifelhaften Bedeutung für diese Untersuchung im Film keine Berücksichtigung, da es in das übergeordnete dramaturgische Gesamtkonzept von Flut 1962 - Erinnern. Gedenken. Erzählen. nicht integriert werden konnte. Dieses Dilemma ist ein Beispiel für die Herausforderungen filmisch-linearer Darstellungskonventionen im Vergleich zu den Möglichkeiten einer , papiergeduldigen 'Verschriftlichung. ${ }^{359}$

\subsubsection{Interviews montieren}

Für die Montage wurden die gefilmten Interviews zwei Arbeitsschritten unterzogen: erstens einer erneuten, minutiösen Auseinandersetzung mit den Interviewtranskriptionen und zweitens einer sukzessiven Verdichtung, oder wie Schröder ana$\log$ zu optischen Verfahren ausführt, einer „Scharfeinstellung“, aus der „innerhalb einer kleinen Gruppe ein subjektiver Erfahrungskosmos ,herangezoomt "“ wurde. ${ }^{360}$ Hierfür wurde das Material thematisch sortiert, auf sich ergänzende oder widersprechende Aussagen hin gruppiert und erneut, nun anhand des entwickelten dramaturgischen Konzeptes, kategorisiert.

Eine weitere Verdichtung der ausgewählten Interviewpassagen erfolgte in der Arbeitsphase des Filmschnitts. Nun wurde deutlich, ob die Zusammenführung zuvor schriftlich transkribierter, miteinander in inhaltlich relevanter Beziehung stehender Interviewaussagen in der Montage umsetzbar sein bzw. in seiner intendierten Aussage- und Vermittlungsabsicht auch funktionieren würde. Ballhaus etwa führt in diesem Zusammenhang die Problematik zweier Protagonist_innen mit „disharmonischen Tonfall[s]" an. ${ }^{361}$ Damit verweist er auf eine zentrale Problematik der Repräsentation gefilmter Interviews im Unterschied zu verschriftlichten sowie insgesamt auf mögliche Auswahlbeschränkungen, die auktorialen Aussageabsichten entgegenstehen können. In einem Film, der fast ausschließlich aus Interviews besteht, kann die Montage zweier Aussagen schon dann den Eindruck von Diskontinuität erzeugen, wenn sie sich hinsichtlich des Tonfalls, des Sprachtempos oder Ähnlichem stark unterscheiden. Diese Problematik trifft für Flut 1962 - Erinnern. Gedenken. Erzählen. jedoch nur begrenzt zu, da der hier intendierten Darstellung individueller, gemeinschaftlich gerahmter Erzählungen durch die hohe Vielzahl

359 Mehr Möglichkeiten bieten, im Vergleich zu linearen Filmen, neuere Formen des non-linearen

Erzählens. Verwiesen sei in diesem Kontext bspw. auf sog. webbasierte $i$-docs, die eher einem

Materialarchiv gleichen und die Nutzer_innen dazu auffordern, sich durch eine Vielzahl meist kurzer Videoclips zu navigieren. Anders als einer von dem bzw. der Filmemacher_in festgelegten Dramaturgie, folgen die Zuschauer_innen hierbei selbst und immer wieder aufs Neue gewählten Pfaden durch das bereitgestellte Filmmaterial. Daher erlaubt eine solche von Produzenten_innenseite bewusst narrationsarm enkodierte Umgebung auch ein viel höheres Maß an (zum Teil auch stark ausdifferenzierten) inhaltlichen Dimensionen. In einer auktorialen linearen Erzählung hingegen sind diese Dimensionen der Gesamtdramaturgie unterordnet bzw. müssen ihr zumindest partiell zwangsläufig ,zum Opfer' fallen.

360 Vgl. Schröder 2009, S. 65.

361 Vgl. Ballhaus 2013b, S. 258. 
unterschiedlicher Protagonist_innen entsprochen wurde. In der Montage wurde somit durch eine schnelle Schnittfolge vieler Einzelaussagen eine Dynamik erzeugt, die die Aufmerksamkeit von Diskontinuitäten abzulenken vermag, selbst wenn sie an einzelnen Schnittübergängen entstanden sind.

Die letztendlich ausgewählten Interviewpassagen wurden „polyperspektivisch“362 und „mosaikartig“ angeordnet. ${ }^{363}$ Mit Fechner kann dieses Vorgehen wie folgt umrissen werden:

„In monatelanger Arbeit wurden dann am Schneidetisch die verschiedenen Einzelinterviews in kleine und kleinste Partikel zerlegt und nach Thema neu geordnet und so aneinandermontiert, daß Aussage und Aussage, Rede- und Gegenrede und Widerspruch, Erzählung auf Erzählung folgten, ein endloser Dialog von Menschen, die sich scheinbar miteinander unterhielten, obwohl sie getrennt voneinander aufgenommen waren." ${ }^{364}$

Dieser Montagestil verschleiert seine Konstruiertheit nicht, sondern ist explizit als „künstlich“365 oder „künstlerisch“366 rezipierbar. Voraussetzung für die Redlichkeit einer solchen Arbeitsweise ist, wie auch Wilbers hervorhebt, dass die gefilmten Menschen tatsächlich in einem „lebensgeschichtlichen Bezug zueinander“" stehen, da diese Art der Montage durchaus suggeriert, dass „eine räumliche und zeitliche Übereinstimmung" besteht. ${ }^{367}$ Auch wenn die Dreharbeiten zu Flut 1962 - Erinnern. Gedenken. Erzäblen. in einem Zeitraum über mehrere Monate an unterschiedlichen Orten stattgefunden hatten, und viele der Befragten einander nicht bekannt waren, so weist dieser Film auch über traditionelle ethnografische Grenzen hinaus, indem sein synchronistisch-beschreibender Duktus durch die Montage dekonstruiert wird und er somit sein, dem Medium inhärentes, durchaus emergentes Potenzial entfalten kann. Die Montage soll hier daher insgesamt als ein heuristisches Werkzeug angesehen werden.

362 Hißnauer 2011, S. 300.

363 Vgl. Wilbers 2001, S. 285.

364 Fechner, Eberhard: Rede anlässlich der PEN-Tagung Erlangen 1978. In: Nagel, Josef/Kirschner, Klaus (Hg.): Eberhard Fechner. Die Filme, gesammelte Aufsätze und Materialien. Erlangen 1984, S. 183-197, S. 192.

365 Wilbers 2001, S. 282.

366 „Genauso wie das wissenschaftliche Schreiben als schriftstellerische Tätigkeit erkannt wurde gilt es, das wissenschaftliche Fotografieren, Filmen, Arbeiten mit Klängen und Geräuschen oder Sammeln und Ausstellen von Objekten als produzierende, kreative Tätigkeit zu verstehen. Dies führt die wissenschaftliche Praxis in das Feld der Kunst mit ihren vielfältigen Formen und Strategien medialer Repräsentationen. Tatsächlich denke ich, dass ein artistic turn in den Kulturwissenschaften wünschenswert und notwendig ist, um die erkenntnistheoretischen Potentiale der verschiedenen Medienformate für die Ethnografie zu erschließen." Overdick, Thomas: Martin Rosswog: ein visueller Ethnograph des ländlichen Europas. In: Simon, Michael u. a. (Hg.): Bilder. Bücher. Bytes. Zur Medialität des Alltags. 36. Kongress der Deutschen Gesellschaft für Volkskunde in Mainz vom 23. bis 26. September 2007. Münster u. a. 2009, S. 173-182, S. 181. 
Nicht erst die Montage, sondern bereits die Dreharbeiten hatten aus mehreren Arbeitsphasen bestanden. Diese erfolgten nicht in zwei schematisch angelegten und in sich abgeschlossenen Feldphasen. ${ }^{368}$ Ballhaus plädiert dafür, die erste Phase als eine reine Forschungsphase ohne Kamera zu konzipieren, und die zweite, die Dreharbeiten, diene demzufolge ausschließlich der filmischen Umsetzung der zuvor erhobenen Erkenntnisse. Ballhaus' Forderung, „alle Elemente (also das während Dreharbeiten produzierte Material) [...] bereits im Feld in ihrer endgültigen und unwiderruflichen Form im Bild zu fixieren "369 vernachlässigt zum einen den Mehrwert einer Flexibilität gegenüber z. B. spontanen Ideen der gefilmten Interviewpartner_innen. Zum anderen würde bei einer solchen Vorgehensweise der für die Filmgestaltung wesentliche Bedeutungsgehalt der Montage - als wenn man so will ,dritter Arbeitsphase - außer Acht gelassen. Neuere methodologische Paradigmen antworten demgemäß auf diese Notwendigkeiten. Sandra Eckardt und Torsten Näser fassen zusammen:

„Während des ineinanderfließenden hermeneutischen Prozesses von Materialsichtung, Auswertung und Schneiden erster Probesequenzen wird das Rohmaterial immer wieder neu in Beziehung zueinander gesetzt. Die daraus gewonnenen Erkenntnisse führen zu einigen weiteren Aufnahmephasen, wodurch der kreative Prozess, der neue Sichtweisen, Erkenntnisse und Ideen hervorbringt, aufrechterhalten wird.“370

Wie oben dargelegt wurde, ist auch Flut 1962 - Erinnern. Gedenken. Erzählen. das Ergebnis eines sowohl hermeneutischen als auch kreativen Prozesses. Die in dem Film zusammengefassten zentralen Ergebnisse dieser Untersuchung werden im Folgenden näher ausgeführt.

368 Vgl. Ballhaus, Edmund: Erkenntnis als Inszenierung. Inszenierung als Erkenntnis. In: Simon, Michael u. a. (Hg.): Bilder. Bücher. Bytes. Zur Medialität des Alltags. 36. Kongress der Deutschen Gesellschaft für Volkskunde in Mainz vom 23. bis 26. September 2007. Münster u. a. 2009, S. 164-172, S. $168 f$.

369 Ballhaus 1995a, S. 17.

370 Eckardt/Näser 2014, S. 286. 


\section{Der Film Flut 1962 - Erinnern. Gedenken. Erzäblen.}

Der Mehrwert des Mediums Film besteht darin, durch sein audiovisuelles Paradigma die, gesellschaftlichen Phänomenen inhärente, Mehrdimensionalität herauszustellen und dabei in einem bewussten Zusammenwirken von Ton- und Bildebenen zu argumentieren. Der Film Flut 1962 - Erinnern. Gedenken. Erzählen. ordnet sich in das Genre ,Interviewfilm' ein, da er primär durch Interviewaussagen konzipiert ist, die einem, hier bereits erläuterten, Analyse- und Auswahlverfahren unterlagen. Nichtsdestotrotz sind im Medium Film „Bilder, Töne und Sprache zu einer sinnlich-suggestiven Einheit verdichtet. ${ }^{\text {“371 }}$ Somit argumentiert auch dieser Forschungsfilm mit Hilfe einer filmsprachlichen Gestaltung, die im besten Fall verstanden wird und dazu auch unterhaltsam ist. Leimgruber, Andris und Bischoff formulieren in diesem Kontext mit Bezug auf MacDougall zielführend:

„Die Herausforderung besteht darin, Formen zu finden, die einerseits auch jenseits eines Fachdiskurses verstanden werden, die aber andererseits in Inhalt und Form mit dem Herkömmlichen in einem kommerziellen wie in einem wissenschaftlichen Sinn brechen." ${ }^{372}$

\footnotetext{
371 Gerndt 2009, S. 321.

372 Leimgruber/Andris/Bischoff 2013, S. 276, mit Bezug auf MacDougall 1998.
} 
Zunächst muss daher auf Besonderheiten dieses Films im Kontext fachlicher Diskurse eingegangen werden, um in kritischer Bezugnahme die von mir gewählten filmischen Narrationsstrategien zu begründen. Diese zielen nicht nur darauf ab, mit dem „Herkömmlichen“ ethnografisch-filmischer Konventionen zu „brechen“, sondern den Konstruktionscharakter des Mediums Film kenntlich zu machen.

Um es vorweg zu nehmen: Die Machart von Flut 1962 - Erinnern. Gedenken. Erzäblen. kollidiert mit innerfachlichen hegemonialen Diskursen über das Wissensformat Film. Näser stellt in seiner Untersuchung der Fachtraditionen und -diskurse des volkskundlichen und des ethnografischen Films fest, dass die normativen Besonderheiten „aus bestimmten Klassifikationstraditionen, eingeübten Prozeduren und institutionellen Rahmungen" resultieren. ${ }^{373}$ Zur Konstruktion filmischer Aussagen nutze ich, wie bereits dargelegt, konstitutiv eine Darstellung der Protagonist_innen als Talking Heads. ${ }^{374}$ Ich spreche ihnen eine audiovisuelle und performative Kraft zu, die von anderen Fachvertreter_innen nicht so stark betont wird. Zudem verzichtet der Film entgegen der ethnografisch-filmischen Konvention auf explizite selbstreflexive Momente, etwa dadurch dass ich als Filmemacherin sicht- bzw. hörbar wäre oder dadurch, mittels einer bewegten Handkamera die Subjektivität der Filmbilder hervorzuheben. ${ }^{375}$ Entgegen dieser Prämisse sind hier die filmischen Aufnahmen ausschließlich mit einem Stativ gedreht. Selbstreflexivität offenbart sich innerhalb der bereits hier explizierten, extradiegetisch „erzählende[n] Instanz" ${ }^{\text {"376 }}$ der Filmemacherin: von der Bildgestaltung und Interviewauswahl bis hin zur die Interviewaussagen organisierenden Montage. ${ }^{377}$ Selbstreflexivität wird hier verstanden als insgesamt volkskundlich-kulturanthropologische Modalität, also auch unabhängig von intendierten filmischen Repräsentationsstrategien.

\section{Filmmusik}

Auf der auditiven Ebene ließen sich - innerfachlichen Diskursen folgend - sowohl der hier gewählte Einsatz von Musik als auch die zum Teil asynchrone Tonmontage als strukturierende und gestaltende filmische Mittel kritisieren. Beide gelten jedoch als dokumentarfilmische Parameter und sind auch für Flut 1962 - Erinnern. Gedenken. Erzählen. essentiell. Es werden drei Musikstücke eingesetzt. ${ }^{378}$

Das Musikstück Minimal 13 von Franziska Wodicka wird im Prolog und im Hauptteil des Films verwendet, in denen die damaligen Geschehnisse thematisiert sind. Es hat ein sehr langsames Tempo mit wenigen Harmonien, es sind aufsteigende Töne, innerhalb eines reduzierten, sich wiederholenden Motivs. Die Modulationen

\footnotetext{
373 Vgl. Näser 2014, S. 15. Zu den Fachtraditionen des volkskundlichen Filmes siehe Leimgruber 2010, S. $13 \mathrm{ff}$. sowie Ballhaus 1987.

374 Siehe hierzu ausführlich Kapitel 3.2.2.

375 Fachinterne Diskurse zur mobilen Handhabung der Kamera siehe Näser 2014, S. $220 f f$.

376 Kiener 1999, S. 175.

377 Vgl. ebd., S. 174.

378 Es handelt sich hierbei um sog. ,GEMA-freie` Musikstücke: www.soundtaxi.de.
} 
halten inne vor jedem Akkord, was eine gewisse Schwere vermittelt. Zudem entfalten die sich wiederholenden Mollklänge den Eindruck, als würde die Zeit abgebremst. Dieses Musikstück möchte den inneren Haltungen der Interviewpartner_innen entsprechen, wenn sie ihre Erfahrungen thematisieren. Durch die leichte Vibration und durch den Nachhall ist aber auch Bewegung assoziiert, die Akkorde entfalten sich. Somit soll impliziert werden, dass kein Stillstand herrscht, denn auch die Befragten verharren nicht in den von ihnen beschriebenen Erlebnissen, sondern bewegen sich erzählend aus ihnen hinaus.

Das Musikstück Nightwish von Jens Fiedler fungiert als leitmotivische Klammerung der persönlichen Masternarrationen im zweiten Filmteil. Es ist ein versöhnliches Thema und vermittelt eine positive Grundstimmung sowie insbesondere einen zeitlichen Abstand und entspricht damit der Haltung der Befragten, die aus heutiger Perspektive erzählen. Zudem sollen das anmutige Klavier und die beruhigende Wirkung von Cello und Geigen assoziieren, dass die Geschichte gut ausgeht. Die Musik entfaltet im Übergang zum dritten Filmteil -zeitgenössischem Filmmaterial, das Aufräumarbeiten zeigt - eine geradezu ,erleichternde` Wirkung und unterstützt so nicht nur den thematischen Wechsel, sondern soll als Angebot an das Filmpublikum verstanden werden, emotionale Entwicklungen der Protagonist_innen mitzuvollziehen.

Das Musikstück Sensitive von Markus Stambader wird im Vor- und Abspann zur Unterlegung von Texttafeln mit Informationsgehalt verwendet. Es ist ein versöhnliches und vermittelndes Thema, atmosphärisch positiv, in Dur. Die Kombination von Klavier und Rhythmusmaschine, zudem als Loop, weist eine große Gleichförmigkeit und Künstlichkeit auf. Dies findet auf der Bildebene eine Entsprechung durch die Verwendung von Texttafeln und unterstreicht somit musikalisch die analytische, extradiegetische Perspektive, durch die diese Filmproduktion bestimmt ist.

Insgesamt dienen die drei Musikstücke einer Akzentuierung der dramaturgischen Kapitelstruktur und als leitmotivische Verklammerung. Die hier also grundsätzlich asynchron verwendete Filmmusik, deren Quelle als sogenannter Soundtrack im Film nicht sichtbar ist, kann als eine „selbstständige Mitteilungsebene“ angesehen werden, da sie Bedeutungen evoziert, Interpretationsweisen nahelegt und beim Publikum Emotionen wecken kann. ${ }^{379}$ Diese Wirkungspotenziale prädestiniert eine Verwendung von Filmmusik allerdings geradezu dazu, innerhalb fachlicher Diskurse unter Manipulationsverdacht gestellt zu werden. Der Medienwissenschaftler Knut Hickethier hält dem nicht nur entgegen, dass „das visuell Gezeigte mit emotionalen Qualitäten versehen"werde, sondern er argumentiert, dass Filmmusik die „im Geschehen angelegte[n] Stimmungen“"verstärken könne. ${ }^{380}$ Diese Funktion hebt auch der Autor und Filmemacher Thomas Balkenhol hervor. Den Mehrwert von Musik innerhalb audiovisueller Vermittlungsstrategien sieht er in ihrem auch

\footnotetext{
379 Vgl. Hickethier, Knut: Film- und Fernsehanalyse. 4., aktualisierte und erweiterte Auflage. Stuttgart 2007, S. 94.

380 Vgl. ebd. Hervorhebung im Original.
} 
interpretierenden Gehalt. ${ }^{381}$ Zudem könne Musik, etwa nach längeren Interviewpassagen, bewirken, das Gesagte „nachklingen“ zu lassen. ${ }^{382}$

Diese explizierenden Wirkungsweisen interpretiere ich als gestalterischen Mehrwert in Hinblick auf das Erkenntnisinteresse und die Vermittlungsabsichten, daher finden neben den Sprachteilen der Interviewausschnitte auch Musikteile konstitutive Verwendung. Sie sind dem zeitgenössischen Foto- und Filmmaterial sowie am Ende des Films heutigen Landschaftsaufnahmen unterlegt. In den Interviewausschnitten sind bereits durch die Art der Erzählweise und durch die Aussageinhalte bestimmte Stimmungen angelegt. Da beabsichtigt ist, dass die Filmmusik diese nicht nur aufgreift, sondern dabei auch vermittelnd und bündelnd wirkt, kann der Filmmusik insgesamt eine die story moderierende Qualität zugesprochen werden.

\section{Asynchrone Tonmontage}

Auf der auditiven Ebene setzt Flut 1962 - Erinnern. Gedenken. Erzäblen. neben der Filmmusik als dramaturgischem Mittel auf eine dezidierte, zum Teil auch asynchrone Tonmontage der Interviewausschnitte. Diese werden mit zeitgenössischem Foto- oder Filmmaterial kombiniert: Wenn im Film etwa die Zeitzeugin Maren Schaub über ihre Erlebnisse in der Nacht vom 16. auf den 17. Februar 1962 erzählt, sieht man zunächst damalige Luftaufnahmen der St. Pankratius Kirche in HamburgNeuenfelde. Diese sind montiert mit heutigen Filmaufnahmen, die Frau Schaub zeigen, wie sie in diese Kirche hineingeht. Aus dem Off - also aufgenommen bei anderer Gelegenheit während des bei ihr zu Hause gefilmten Interviews - beschreibt sie die damalige Situation auf der Empore, wo sich die Menschen nachts Schutz suchend zusammengefunden hatten. Im Film ist zu sehen, wie sie, nun begleitet von der Kamera, wieder die Treppe dort hinauf steigt.

Diese Art der asynchronen Tonmontage - das Bild ist nicht durchgängig mit dem Originalton unterlegt - hat in seiner Wirkung eine Aufhebung von RaumZeit-Synchronitäten zur Folge. Es entsteht somit eine andere Ordnung der Informationsvermittlung: Die Befragten erzählen nicht nur Geschichten („das habe ich erlebt"), sondern dieses filmische Mittel zielt darauf darzustellen, dass die Erzählungen reflektiert und kontextualisiert („deshalb erinnere ich mich daran“) sind. „Episodische Momente ${ }^{\text {(383 }}$, die im Off mit Interviewausschnitten unterlegt sind, finden daher in allen drei Teilen des Films Verwendung. In diesen Sequenzen werden Fotoalben betrachtet, wird der damalige Zufluchtsort aufgesucht, oder am damaligen Wohnort (Garten-)Arbeit erledigt bzw. während des Spaziergangs auf einer Bank sitzend innegehalten.

\footnotetext{
381 Vgl. Balkenhol, Thomas: Pflicht und Kür der Dokumentarfilm-Montage. „24 Mal Wahrheit in der Sekunde" (Godard) - Der Umgang mit der Realität in der Montage von Dokumentarfilmen. In: Beller, Hans (Hg.): Handbuch der Filmmontage. München 1993, S. 123-143, S. 138.

382 Vgl. ebd., S. 139.

383 Vgl. Eckardt/Näser 2014, S. 287.
} 
Das zeitgenössische Film- und Fotomaterial ist innerhalb einer asynchronen Tonmontage zwar teilweise mit Musik unterlegt, nicht jedoch mit Geräuschen, wie es den Konventionen etwa in Fernsehdokumentationen und -reportagen entsprechen würde. ${ }^{384}$ Auch wenn Filmmaterial bis in die 1960er Jahre, also bis zum Aufkommen tragbarer Synchrontonaufnahmemöglichkeiten ${ }^{385}$ und im Amateurbereich zeitlich darüber hinaus, ebenfalls originär ohne Ton gewesen ist, so wurde es seit Beginn filmischer Repräsentation, also mit dem Stummfilm, zusammen mit Ton gezeigt, sei es mit Musik, Sprache oder Klängen. Die Audiovisualität wurde somit erst hergestellt. Auch das hier verwendete Material von 1962 hatte ursprünglich keine Tonspur. ${ }^{386}$ In Flut 1962 - Erinnern. Gedenken. Erzählen. ist dem zeitgenössischen Film- und Fotomaterial entweder Musik oder sogenannte ,Atmo' unterlegt, also eine Tonspur, auf der räumliche ,Stille' aufgenommen wurde, um in der Rezeption die Wirkung eines Tonlochs, also eines filmtechnischen Fehlers, zu vermeiden.

Die Abwesenheit von unterlegten Geräuschen ermöglicht es, sich auf das Visuelle $\mathrm{zu}$ fokussieren und eröffnet somit einen Vorstellungsraum, der in anderen Dokumentarfilmen zumeist durch beigefügte Geräusche schon mitgestaltet und somit vorgegeben wird. Die Stille wird dem gegenüber so zu einem Möglichkeitsraum des Innehaltens. Fotografien und Filmaufnahmen von 1962 ohne Geräuschunterlegung sind daher hier als Angebote an das Publikum zu verstehen, eigene auditive Assoziationen, etwa Hubschrauberrotorengeräusche, Ruderkommandos oder Stimmen und Rufe zu imaginieren. Es sind atmosphärische Partikel, die als impliziter Bestandteil der Interviewaussagen interpretiert, nun im Film mit den Vorstellungen des Publikums korrespondieren sollen.

\section{Zeitgenössisches Foto- und Filmmaterial}

Grundsätzlich dient das für Flut 1962 - Erinnern. Gedenken. Erzählen. verwendete zeitgenössische Foto- und Filmmaterial zur Unterstützung der filmischen Aussageund Vermittlungsabsichten. Fotografien sind nur spärlich eingesetzt, um dem Ein-

384 Filmaufnahmen etwa, die während des Zweiten Weltkriegs entstanden sind, werden häufig nachvertont. Bekannte Geräusche sind beispielsweise marschierende Stiefel, das Krachen von Bombeneinschlägen oder das Klappern von Panzerketten. Dabei handelt es ich um heutige Nachvertonungen, da das ursprüngliche Filmmaterial ohne Ton aufgenommen worden ist.

385 Zur Bedeutung der Synchrontonkamera für die Dokumentarfilmgeschichte des Direct Cinema und des Cinéma Vérité siehe Böhl, Michael: Entwicklung des ethnographischen Films. Die filmische Dokumentation als ethnographisches Forschungs- und universitäres Untersuchungsmittel in Europa. Göttingen 1985, S. 104ff. Kamera und Tonband waren in den 1960er Jahren (und im privaten Videobereich bis in die 1980er Jahre) noch zwei getrennte Geräte, die, mit einem Kabel verbunden, eine Synchrontonaufnahme ermöglichten.

386 Ausschnitte aus dem folgenden zeitgenössischen Filmmaterial von 1962 fanden im Film Verwendung: Flutkatastrophe in Hamburg (1962), Landesbildstelle Hamburg (LBH: Sign. 4200796), Die große Flut (1962), Baubehörde Hamburg - Tiefbauamt (LBH: Sign. 4200128) sowie Privataufnahmen des für diese Untersuchung interviewten Zeitzeugen Horst Stanik. 
zelbild eine größere Wirkung zu ermöglichen ${ }^{387}$ sowie um die Interviewaussagen in den Mittelpunkt des Filmes zu stellen. Die insgesamt 15 Privatfotografien akzentuieren primär die dramaturgische Struktur. ${ }^{388}$

Die zeitgenössischen Filmaufnahmen wurden nicht von Privatleuten, sondern nahezu allesamt von professionellen Kameramännern gedreht. Im Schnitt von mir neu kompiliert, zeigen sie im Gegensatz zu den lokal begrenzten Blick-, respektive Erfahrungsräumen der Befragten verschiedene und einen regionalen Überblick vermittelnde Perspektiven auf: Hubschrauberflüge über die überfluteten Gebiete und Deichbruchstellen etwa sollen es einem heutigen Publikum ermöglichen, einen Eindruck über das Ausmaß der damaligen Überschwemmungen zu erhalten.

Die Verwendung von zeitgenössischem Foto- und Filmmaterial ist zwar als Bestandteil dokumentarfilmischer Konventionen innerhalb wissenschaftlicher Paradigmen akzeptiert. ${ }^{389}$ Nichtsdestotrotz haben normative Vorbehalte innerhalb ethnografisch-filmischer Diskurse auch hier eine offensive Verwendung dieser filmsprachlichen Mittel bisher eingeschränkt. Als diskursiv legitime filmische Form gelte es stattdessen, nicht mit historischen, sondern mit „den gegenwärtig in der Wirklichkeit vorfindbaren Bildern zu operieren“, wie Näser mit Bezug auf Tobias Wendl hervorhebt. ${ }^{390}$

Mit dem Film Flut 1962 - Erinnern. Gedenken. Erzählen. positioniere ich mich somit in mancher Hinsicht in Abgrenzung zu fachlichen Diskursen, die eine bestimmte normative Machart für Filme vorzugeben scheinen. Stattdessen möchte ich dafür werben, die Potenziale filmischer Konventionen, die außerhalb fachlicher Vorbehalte längst erprobt und Teil dokumentarfilmischer Praxisdiskurse sind, für volkskundlich-kulturanthropologisches Filmschaffen verstärkt im Sinne einer extension of eye and mind zu nutzen.

Im Folgenden möchte ich anhand der dreiteiligen Struktur von Flut 1962 - Erinnern. Gedenken. Erzäblen. die darin jeweils thematisierten analytischen Schwerpunkte und Ergebnisse dieser Untersuchung ausführen: Die Relevanz von Fotografien bzw. von Fotoalben für die Tradierung der Erzählungen (Kapitel 4.1), die strukturellen und inhaltlichen Besonderheiten der persönlichen Masternarrationen (Kapitel 4.2) sowie die (Selbst-)Repräsentation der Zeitzeug_innen im Kontext heutiger Diskurse von Vulnerabilität und Resilienz (Kapitel 4.3).

387 Vgl. Gerndt 2009, S. 317.

388 Fotonachweis: Geschichtswerkstatt Wilhelmsburg \& Hafen; Heimatmuseum Wilhelmsburg; Kulturkreis Finkenwerder; Jutta Dierks; Gerhardt Wendt.

389 Vgl. Ballhaus 2014, S. 259f.

390 Vgl. Näser 2014, S. 332, mit Bezug auf Wendl, Tobias: Perspektiven der Visuellen Anthropologie. In: Wissenschaftlicher Film 44, 1992, S. 107-120, S. 112. 


\subsection{Filmteil 1: Erzählen mit zeitgenössischen Fotografien}

In die Interaktion mit den Interviewpartner_innen war ersichtlich geworden, dass den privaten Fotografien und Fotoalben eine große Bedeutung für die Tradierung ihrer Erzählungen zukommt.

Im Folgenden werden zunächst die damaligen Bedingungen, mit denen sogenannte Medienamateur_innen konfrontiert waren, nachvollzogen sowie deren konkrete Fotomotive näher ausgeführt werden. Anschließend wird das heutige Erzählen anhand von zeitgenössischen Fotografien und mit Fotoalben als filmischer Einstieg im ersten Filmteil plausibilisiert.

\subsubsection{Medienamateur_innen und ihre Fotomotive}

Als in der Nacht vom 16. auf den 17. Februar 1962 die Deiche brachen, hatten die Menschen zunächst sicherlich anderes im Sinn, als zu fotografieren. Sie kamen vermutlich gar nicht auf die Idee, weil sie um ihre materielle und physische Existenz kämpften. Darüber hinaus erschwerten es die fototechnischen Voraussetzungen der Kleinbildkameras in der nächtlichen Dunkelheit, bedingt zudem durch den damaligen Stromausfall, Aufnahmen zu machen. Die meisten der überlieferten Fotografien entstanden vor allem mit dem Tageslicht ab dem 17. Februar 1962, aufgenommen zumeist von denjenigen, „die über einen ,schussbereiten 'Fotoapparat verfügten und die außerdem aufgrund der Überflutungen ihre Häuser nicht verlassen konnten“ bzw. in höhere Stockwerke geflüchtet waren. ${ }^{391}$ Damit lässt sich die häufige Vogelperspektive der Fotografien erklären. ${ }^{392}$ Aufgenommen wurden, „die ersten Eindrücke [im] unmittelbaren Nahbereich: sichtbare Schäden, Wassermassen und -stände, die Häuser in der Nachbarschaft sowie Anstrengungen von Helfern, etwa Rettungseinsätze per Hubschrauber oder Sturmboot. “393

Bedauerlicherweise ist häufig über die Menschen, die damals fotografierten, wenig bekannt, und ihre Beweggründe für das Fotografieren sind kaum noch ermittelbar, sobald sich die Fotos nicht mehr im Besitz der damaligen Fotograf_innen befinden. ${ }^{394}$ Die Relevanz ihrer ,Schnappschüsse ist jedoch nachhaltig bis in die

391 Vgl. Paech 2008, S. 167.

392 Im Forschungsverlauf konnten etwa 700 Amateurfotografien von Zeitzeug_innen und aus lokalen Archiven zusammengestellt und digitalisiert werden. Eine systematische fotoanalytische Auswertung der Fotografien erfolgte nicht. Private Filmaufnahmen sind indes sehr selten, auch weil dies damals ein kostenintensives Hobby war. Ein Modell zur Analyse von Fotografien, mit Bezug auf Erwin Panowsky und Roland Barthes, siehe Hägele, Ulrich: Visual Folklore. Zur Rezeption und Methodik der Fotografie In: Göttsch, Silke/Lehmann, Albrecht (Hg.): Methoden der Volkskunde. Berlin 2001, S. 277-300, S. 290ff.

393 Paech 2008, S. 167.

394 Vgl. das Fotobeispiel bei Regener, Susanne: Medienamateure - Fotografie und soziale Praxis im Alltag. In: In: Helmut Gold u. a. (Hg.): DIY. Die Mitmach-Revolution. Katalog zur Ausstellung. Frankfurt a. M. 2011, 176-187. Die Fotografie befindet sich im Archiv der Geschichtswerkstatt Wilhelmsburg \& Hafen. Dort lagen keine das Foto kontextualisierende Informationen vor. Der Fotograf wird von Regener daher in der Publikation als „anonym“ bezeichnet. 
heutige Zeit, wie auch Susanne Regener betont, denn diese „visuellen Praktiken produzieren neue Zeichen und Codes für Authentizität und Identität. Sie stellen damit Alltagswirklichkeit her und lassen die Bilder in einem globalen Rahmen kommunizieren. "395 Bestimmte Bildmotive haben eine frappierende Ähnlichkeit mit heutigen, wie etwa Aufnahmen von überfluteten Gebieten oder von Zerstörungen. Diese Bildtypen scheinen zeitlos und global verständlich zu sein. Für die Amateurfotografien zur Hamburger Sturmflut von 1962 stellt sich daher auch für Regener die Frage: „Sind diese Fotos Teil einer Ikonografie der Katastrophe?"396

Diese Medienamateur_innen waren damals die ersten, weil schon vor Ort, die Fotos ,knipsten', zumeist aus dem privaten Wohnumfeld bzw. dem „rettenden Unterschlupf ${ }^{\mathrm{r}}{ }^{397}$ heraus. ${ }^{398}$ Pressefotografen kamen erst zusammen mit den Rettungsteams in die betroffenen Gebiete und hielten sich im Gegensatz dazu im öffentlichen Raum auf. ${ }^{399}$ Viele der Privatfotografien, die im Februar 1962 aufgenommen worden waren, sind häufig, vermutlich besonders innerhalb von Nachbarschaften, ausgetauscht worden. Ein Beleg für diese ,soziale Gebrauchsweise ${ }^{6400}$ ist, dass dieselben Aufnahmen als Abzüge in unterschiedlicher Papierqualität und Größe sowohl bei verschiedenen Gesprächspartner_innen als auch in lokalen Archiven - hier häufig als (Nachlass-)Schenkungen - zu finden sind. ${ }^{401}$ Die nachbarschaftliche Unterstützung hatte während der Flutnacht begonnen und sich zeitlich bis zu gemeinschaftlichen Aufräumarbeiten in den nachfolgenden Tagen und Wochen fortgesetzt. Somit erscheint der Austausch von Fotografien wie die Weiterführung eines Gemeinschaftssinns. Diese Praxis kann neben dem Austausch von Erzählungen darüber hinaus als ein Motiv gedeutet werden, um soziale Beziehungen zu vertiefen. ${ }^{402}$

395 Ebd., S. 178. Regener hebt insgesamt für die fotografischen Praktiken während der Hamburger Sturmflut von 1962 die Bedeutung der Medienamateur_innen hervor. Vgl. ebd., S. 176.

396

397 privaten Fotografien. Vgl. Bourdieu, Pierre u. a. (Hg.): Eine illegitime Kunst. Die sozialen Gebrauchsweisen der Fotografie. Frankfurt a. M. 1981. Eine konzise Einordnung dieser Studie siehe Mathys, Nora: Fotofreundschaften. Visualisierungen von Nähe und Gemeinschaft in privaten Fotoalben aus der Schweiz 1900-1950. Baden 2013, S. $34 f$.

401 Beispielsweise in der Geschichtswerkstatt Wilhelmsburg \& Hafen und im Archiv des Kulturkreises Finkenwerder e. V.

402 Zur Bedeutung dieser Tauschpraktiken führt Elisabeth Edwards aus: „The exchange of the photograph as image itself expresses the social value of the relationship that is maintained and sustained between groups and individuals, which demand reciprocity to consolidate the socially desired 
Unmittelbar nach den Ereignissen kam den Fotografien vermutlich darüber hinaus die Aufgabe zu, Sinn herzustellen. ${ }^{403}$ Als damalige Motivation für das Fotografieren kann daher gelten, das Unvorstellbare haptisch durch die Fotografie greifbar und somit begreifbar werden zu lassen. Heutzutage können sie als Ausdruck eines „desire for memory “ 404 interpretiert werden, also dem Wunsch danach, nicht zu vergessen. Damit einher geht eine Wertschätzung von Erinnerungen. Fotografien eröffnen selbstreflexive Rückbezüge bei der Auseinandersetzung mit Vergangenheit und deren Vergegenwärtigung. ${ }^{405}$ In diesem Zusammenhang steht eine heutige Nutzung als zeitlicher Vergleich. Als Referenz dienen hierbei die abgebildeten Orte in ihrem gegenwärtigen Zustand. Somit ist bei der heutigen Betrachtung von Fotografien, auf denen Zerstörungen abgebildet sind, implizit eingeschrieben, dass die damaligen Schäden beseitigt worden sind. Ein konnotativer Gehalt der Fotografien stellt somit die Erfahrung von Katastrophenbewältigung dar. Vor diesem Hintergrund sollen im Folgenden die in Flut 1962. Erinnern. Gedenken. Erzählen. dargestellten „sozialen Gebrauchsweisen“" ${ }^{406}$ von Fotografien in Fotoalben als einem wesentlichen Erzählanlass näher betrachtet werden.

\subsubsection{Fotoalben als Erzählanlass}

Fotoalben dienen Menschen dazu, persönliche Erinnerungen zu ordnen. In diesem Sinne können sie als Ausdruck lebensgeschichtlicher Repräsentation der ,Albenautor_innen ${ }^{407}$ angesehen werden. ${ }^{408}$ Durch die Auswahl und die zumeist chronologische Anordnung der Fotografien inklusive der Beschriftungen, also durch Inhalt, Form und Materialität, ist in ihnen eine Geschichte ,eingeklebt', die nun mit Hilfe des Albums abgerufen werden kann. ${ }^{409}$ Während bspw. ein Schuhkarton als

memory of images. Thus the implications of the gifting relationship are integral to the meaning of the photo-object in gestures which recapitulate or re-enact social articulations. They reinforce networks and identity built on the memory to which they relate, positioning individuals vis à vis the group, linking past, present and perhaps implying a future." Edwards, Elisabeth: Photographs as Objects of Memory. In: Kwint, Marius/Breward, Christopher/Aynsley, Jeremy (Hg.): Material Memories. Oxford/New York 1999, S. 221-236, S. 233. Hervorhebung im Original.

403 „Das Festhalten des Augenblicks attestiert dem Geschehen ,Sinn“ und überführt es in die Potenzialität, Geschichte zu werden. "Köstlin, Konrad: Photographierte Erinnerung? Bemerkungen zur Erinnerung im Zeitalter ihrer technischen Reduzierbarkeit. In: Brunhold-Bigler, Ursula/Bausinger, Hermann (Hg.): Hören Sagen Lesen Lernen. Bausteine zu einer Geschichte der kommunikativen Kultur. Festschrift für Rudolf Schenda zum 65. Geburtstag. Bern 1995, S. 395-410, S. 404.

404 Vgl. Edwards 1999, S. 222.

405 Vgl. Assmann 2013, S. 206.

406 Vgl. Bourdieu 1981

407 Zum Begriff des ,Albenautors'sowie ein Überblick über aktuelle Forschungsarbeiten zu privaten Fotografien siehe Mathys 2013, insbes. S. 34-38 und S. 45-48.

408 Starl spricht umfassender von „einem lebensgeschichtlichen Entwurf“, der sich in der Art und Weise, wie Fotografien ausgewählt, angeordnet und ggf. mit Bildunterschriften versehen werden, ausdrückt. Vgl. Starl 1995, S. 22 f.

409 Vgl. Köstlin 1995, S. 403; vgl. Mathys 2013, S. 42. 
Behältnis die Abgeschlossenheit, Intimität oder Unsortiertheit einer Erfahrung assoziieren könnte, so ließe sich das Fotoalbum zum einen als - stets griffbereites Repräsentationsmedium kennzeichnen. Konrad Köstlin führt aus: „In diesem Privatkult (der so privat, wie er sich gibt, nicht ist) stellen wir biographische Arrangements nicht nur fürs Gedächtnis, sondern auch zum Vorzeigen her. " ${ }^{410} \mathrm{Zum}$ anderen verarbeiten Menschen durch die Sammlung und Sortierung, also durch eine „aktive Organisation“ gegen das Vergessen und für eine Sinngebung sowie insbesondere durch ihre konstruktive Fixierung in einem Fotoalbum, ihre Erfahrungen, indem sie ihre Erlebnisse in eine "feste Reihenfolge" bringen. ${ }^{411}$

Somit kann die Erstellung von Fotoalben über die Hamburger Sturmflut von 1962 als Ausdruck einer Haltung interpretiert werden, die Ordnung in die Erfahrung von Chaos transformieren möchte. Dabei ist es wesentlich, dass die Fotografien objektiv als wahr interpretiert werden, um zu einer biografisch gelungenen, also konsistenten Bilanzierungsarbeit gelangen zu können. ${ }^{412}$

Die sortierte Zusammenstellung in Fotoalben bleibt stets unvollständig, denn nicht alles, was erinnert wird bzw. zu dem Geschichtsnarrativ beiträgt, ist auf den Fotografien abgebildet bzw. überhaupt abbildbar. Trotz dieser fragmentarischen Auswahl sind die Fotografien Erinnerungsobjekte, die im Kontext eines „larger biographical whole of which they are part" interpretiert werden können. ${ }^{413}$

Die hier angeführten Bedeutungen von Fotografien und von Fotoalben sind im ersten Teil des Films dargestellt. Dabei werden zwei erinnerungskulturelle Modalitäten thematisiert: zum einen der innerfamiliäre Austausch, zum anderen eine primär personalisierte Gebrauchsweise, mit der auch der Film beginnt: Gerhardt Wendt, Blumenhändler und Familienvater aus dem Wilhelmsburger Ortsteil Kirchdorf, blättert durch sein Fotoalbum und erklärt in priorisierender und das Erzähltempo variierender Weise, was er sieht bzw. was wir sehen sollen. Seiner Meinung nach „nicht so interessant[e]“ Fotografien überspringt Herr Wendt, und er hält inne bei der für ihn wichtigsten Fotografie, auf der, wie er erklärt, die emotionalen Erschütterungen seiner Angehörigen festgehalten sind.

410 Köstlin 1995, S. 397. In beiden Fällen ermöglichen es diese „kompakten Geschichtenreservoirs“ (Gerndt 2009, S. 318), Erfahrungswissen, respektive Erzählungen, über die Fotoinhalte zu konservieren und abzurufen.

411 Selke, Stefan: Private Fotos als Bilderrätsel - Eine soziologische Typologie der Sinnhaftigkeit visueller Dokumente im Alltag. In: Ziehe, Irene/Hägele, Ulrich (Hg.): Fotografien vom Alltag - Fotografieren als Alltag. Tagung der Kommission Fotografie der Deutschen Gesellschaft für Volkskunde und der Sektion Geschichte und Archive der Deutschen Gesellschaft für Photographie im Museum Europäischer Kulturen - Staatliche Museen zu Berlin vom 15. bis 17. November 2002. Münster 2004, S. 49-74, S. 62. Starl hebt hervor: „Von Bedeutung ist weniger die Fülle des Festgehaltenen als die Reihung der Abzüge im Album.“ Diese bilde „die Stichworte, die der Autor und Arrangeur der Fotografien zum Entwurf der Geschichte seines Lebens benötigt." Starl 1995, S. 23.

412 Vgl. Selke 2004, S. 72; vgl. Köstlin 1995, S. 401.

413 Vgl. Kirshenblatt-Gimblett, Barbara: Objects of Memory. Material Culture as Life Review. In: Oring, Elliot (Hg.): Folk Groups and Folklore Genres. A Reader. Logan 1989, S. 329-338, S. 331. 
Die im Folgenden beschriebene Filmsequenz ist das Ergebnis einer Montage aus mehreren Interviewausschnitten. Die darin von mir gesetzten Ton- und Bild-Schnitte sind durch „[...]“ kenntlich gemacht. Diese Kenntlichmachung im Text gilt ebenso für alle nachfolgenden Interviewauszüge. In dieser Sequenz nun ist Herr Wendt zunächst zu sehen, wie er in seinem Fotoalbum blättert, in halbnaher, frontaler Einstellung sowie über die Schulter gefilmt. Seine Stimme, aufgenommen während des Interviews, ist hierbei in asynchroner Tonmontage ins Off gelegt. Erst in der dritten Einstellung dieser Sequenz ist er in der Interviewsituation, also im $O n$, zu sehen. Seine Beschreibung der Fotografien sind im Film folgendermaßen zusammengefasst:

„Und das ist eine Tankstelle auch bei uns in unmittelbarer Nähe, die dann nur vom Bundeswehrboot angefahren wurde. Und hier oben war ein Punkt, der also dann trocken war, aber das war die Straße hier runter, wo auch denn hier unten der Schlachter ist, und ich hatte noch einmal- Ach nee, das ist auch noch diese Straße. Mmh, das ist also nicht so ganz interessant. Und das ist noch mal bei uns in unserem Schuppen, wie's so aussah, und das ist ein, mmh, Bild (Stimme leiser, Wechsel zum Interview) [...] Zum Glück hatte ich also während der Flutnacht einen Fotoapparat schussbereit im Haus, [...] und konnte also Fotos machen hier bei uns aus dem Fenster raus, wie das Wasser so richtig am höchsten stand. [...] Und auch Nachbarn, die also mir nachher Bilder gegeben haben, die also in derselben Situation waren, die auch wirklich im Wasser standen, und in der Situation noch Fotos gemacht haben von meinem Haus, wie die Bundeswehr hier anlegt mit'm Boot und wie das Wasser richtig bei uns so 20 Zentimeter über der Fensterbank stand. [...] Und äh, es, es war eigentlich schon sehr dramatisch. Also von, dass wir also nervlich auch sehr belastet waren, und wenn Sie sich mal Bilder angucken, die wir also haben, die wir hier gemacht haben in der kaputten Wohnung, wo meine Großmutter, die denn, also, äh, fast 80 war, und meine Mutter, wie die also hier in der Stube sitzen vor den ganzen zerstörten Möbeln und diesen ganzen Bildern, und Sie gucken in diese Gesichter, dann können Sie ungefähr ermessen, wie uns also hier so zumute war."

Am Beispiel von Gerhard Wendt zeigt sich, dass die Fotografien nicht nur einer Illustrierung des Gesagten dienen, sondern es werden „Wirkungszusammenhänge, Zeitabläufe und Raumkonstellationen in visuelle Formen transportiert“. 414 Diese Filmsequenz zeigt zudem, dass die Fotografien hier erst durch die subjektive Erzählung Bedeutung erlangen bzw. dass darin vermittelt und erkennbar wird, welches die für Herrn Wendt relevanten Fotografien sind. ${ }^{415}$ In dieser Sequenz wird somit der

\footnotetext{
414 Gerndt 2009, S. 314.

415 „In der Regel muss man auf Erzählbildern etwas Konkretes identifizieren können, doch wenn die verbildlichte Geschichte geläufig ist, kann sie auch mittels relativ abstrahierter Bilder wiedergegeben werden." Ebd., S. 316.
} 
Bilderserie eine besondere Aussagekraft zugesprochen. ${ }^{416}$ Der Protagonist ist allein in einem Sessel sitzend inszeniert, in Adressierung nach außen an ein Publikum.

In der zweiten Sequenz dieses ersten Filmteils von Flut 1962 - Erinnern. Gedenken. Erzählen. ist ein nach innen gerichteter Dialog dargestellt, der eine weitere erinnerungskulturell relevante Praxis zeigt. ${ }^{417}$ In einer nachgestellten, also bewusst inszenierten Szene nehmen Elsbeth Meyer aus Hamburg-Francop und ihre beiden Töchter, Jutta Dierks und Gitta Rosenau, ein Fotoalbum zum Anlass, miteinander ins Gespräch zu kommen, oder mit Köstlin resümiert: „Am Album entzündet sich die Kommunikation, an ihm wird der homo narrans aktiv, der sich in Bildergeschichten erzählt." ${ }^{118}$ Die Betrachterinnen des Albums tragen in dieser Szene miteinander ihre auch unterschiedlichen Erfahrungen, Wissensstände und Assoziationen zusammen. Sie werden im Gespräch mit in die Geschichte der jeweils Anderen einbezogen. ${ }^{419}$ Die Protagonistin Gitta Rosenau reflektiert im Interview über diese Situation:

„Und es ist auch heute immer noch so, dass immer noch mal die Flut auf 'n Tisch kommt, wenn man mal irgendwo sitzt und man hat irgendwo nen Gesprächsthema oder so, und irgendwo kommt man immer mal wieder da drauf zu sprechen. Und dann wundert man sich eigentlich, was man so, oftmals sagt man, Kinder vergessen vieles, aber solche Ereignisse, wie unsere Eltern eben halt früher den Krieg, da können die auch immer noch von erzählen, und ich denke mal, das sind so Einschnitte im Leben, die vergisst man auch nicht. [...] Aber dass wir das jetzt so unbedingt nach draußen tragen oder so, nö, also dann wird das hier schon innerhalb der Familie besprochen oder mal Erinnerungen ausgetauscht."

Nicht nur Bilderserien, wie an den beiden Beispielen dargestellt, evozieren Erinnerungsleistungen, auch einzelne Fotografien können einen großen Bedeutungsgehalt aufweisen, wie bereits anhand des Fotos von Gerhardt Wendt, das seine Angehörigen zeigt, deutlich wurde. ${ }^{420}$ Am Beispiel der Erzählungen von Familie Meyer veranschaulicht ebenfalls eine Einzelaufnahme, nämlich ihres zerstörten Wohnhauses, unter dem Stichwort „Puppenstube“, dass bestimmte Wissensbestände den Blick lenken können und einem Foto einen ikonischen Gehalt einzuschreiben vermögen. ${ }^{421}$ Gezeigt wird im Film stellvertretend für Familie Meyer die Aussage des Sohns bzw. Bruders Bernd Meyer:

416 Zur seriell-vergleichenden Analyse größerer Bildmengen am Beispiel privater Fotoalben vgl. Mathys 2013, S. 43.

417 Vgl. Ballhaus 2003, S. 38ff.

418 Köstlin 1995, S. 400.

419 Das Fotoalbum gehört Jutta Dierks. Die Fotografien, die das zerstörte Wohnhaus zeigen, hatte sie von einem Architekten erhalten, für den sie beruflich tätig war.

420 Vgl. Gerndt 2009, S. 314.

421 Zudem greifen bei diesem Beispiel „historische [...] medienimmanente [...] Mechanismen“ (ebd.), da zeitgenössische Filmaufnahmen von diesem Wohnhaus häufig in Fernsehreportagen und in Bildbänden abgebildet sind. 
„Das Bild, das vergisst man nicht. [...] Unser Haus, was aussah von der Seite aus nachher, wie eine Puppenstube. [...] Also ich könnte heute ein Blatt hernehmen und das Haus aufmalen, aus'n Kopf und wo jedes Zimmer war, und das vergisst man auch nicht wieder."

In Flut 1962 - Erinnern. Gedenken. Erzählen. korrespondieren die beiden beschriebenen Sequenzen als szenisch-episodische Darstellungen darüber hinaus mit weiteren materiellen Besonderheiten der thematisierten Fotoalben, diesen „kompakten Geschichtenreservoirs“ ${ }^{422}$ : Sie können sowohl großformatig und schwer sein, und die Erinnerungen werden raumgreifend sichtbar für mehrere Personen gleichzeitig ermöglicht oder sie sind in ihrer äußeren Erscheinung unscheinbar und kleinformatig, sodass sich das Album primär für eine individuelle Handhabung anbietet. Somit können die Fotoalben in ihrer Materialität unterschiedliche Haltungen der Adressierung und Vermittlung evozieren. Edwards stellt heraus: „Materiality is also integrally linked to social ways of viewing and thus accessing the past, personally or collectively. “ 423

Dieser Zugriff auf die Erinnerungserzählungen wird durch eine weitere materielle Eigenschaft von Fotoalben buchstäblich zeitlich strukturiert. Neben dem Umstand, dass Fotos in Alben so ausgewählt, angeordnet und die Seiten ggf. mit Text versehen sind sowie dass Erfahrungen retemporalisiert und durch Auslassung und Verdichtungen von Motiven ein Geschichtsnarrativ konstruiert werden, wie bereits dargelegt wurde, so ist hier auch die konkrete Handhabung der Alben von Interesse und somit im ersten Filmteil thematisiert. Gemeint ist die haptische Praxis des Umblätterns und des Verweilens auf Albumseiten. Die Protagonist_innen verdeutlichen damit subjektive Priorisierungspraktiken an dieses Extremereignis. ${ }^{424}$ Einzelne Fotografien bzw. Albumseiten haben scheinbar unterschiedliche Wertigkeiten, wie auch in der oben beschriebenen Sequenz mit Gerhard Wendt deutlich wird, und ihnen wird erst im Zusammenwirken mit als weniger bedeutsam erachteten Fotografien eine Bedeutung zugesprochen.

Die Relevanz einzelner Fotografien für ihre Besitzer_innen wird also erst durch die Erzählungen deutlich. Mehr noch stellt dieser Zusammenhang heraus, dass dieses „Reden über Erfahrung “ 425 mit Fotografien und Fotoalben als eine wichtige erinnerungskulturelle Praxis, besonders innerhalb von Familien, angesehen werden kann. Sie bilden Erinnerungsanker für die zentralen Geschichten aus, und die Fotografien werden durch die Erzählungen „verlebendigt“ ${ }^{426}$. Somit wiederum stimulieren sie Erzählungen, dienen aber gleichzeitig ihrer Lenkung, insbesondere aufgrund der ausgestal-

\footnotetext{
422 Ebd., S. 318.

423 Edwards 1999, S. 228.

424 Vgl. ebd., S. 230.

425 Lehmann 2007.

426 Gerndt 2009, S. 313. Christian Volmari führt aus: „Denn im Gegensatz zu dem fotografierten Objekt bleibt eine Stimmung abstrakt, nicht sinnlich erfahrbar und somit nur über den Umweg
} 
teten Rahmung in den Fotoalben. Diese „innere Ordnung der Alben “427 ist für diese Untersuchung besonders wichtig, korrespondiert sie doch mit dem ordnenden Charakter von Erzählstrukturen und -mustern sowie mit inhaltlichen Schwerpunktsetzungen der Interviewpartner_innen. Im zweiten Filmteil werden diese Analyseergebnisse, erhoben insbesondere anhand der persönlichen Masternarrationen, dargestellt.

\subsection{Filmteil 2: Erzählen als persönliche Masternarration}

Wie bereits im Kapitel 1.2 angedeutet wurde, vermittelten die Befragten nicht den Eindruck, dass sie sich in den Interviews zum ersten Mal zu den Geschehnissen äußern, sondern dass ihre Erlebnisse bereits gegenüber Familienangehörigen, Freund_innen oder Nachbar_innen formuliert bzw. untereinander ausgetauscht und tradiert worden sind. Dies betrifft insbesondere die zu Beginn der Interviews jeweils erfragten Erlebnisschilderungen. Diese Haupterzählungen wurden von den Zeitzeug_innen strukturiert wiedergegeben. Dabei orientierten sie sich an von ihnen bereits erprobten Erzählmustern und an gesellschaftlichen Erzählkonventionen, also an der in der Gesellschaft verankerten Meistererzählung, über die damaligen Ereignisse.

Der Film Flut 1962 - Erinnern. Gedenken. Erzählen. stellt einzelne Protagonist_innen in den Dienst einer Gesamterzählung. ${ }^{428}$ Der zweite Teil des Films ist in seiner Montage am stärksten darauf ausgerichtet. Im Folgenden werden daher die wesentlichen Analyseergebnisse einer transdisziplinär argumentierenden Erzählforschung als Bewusstseinsanalyse vorgestellt, die diesem Filmteil zugrunde liegen. ${ }^{429}$

\subsubsection{Muster des Erzählens}

Wesentlich strukturiert sind die hier analysierten autobiografischen Erzählungen durch thematische Leitlinien. ${ }^{430}$ Ihnen gilt zunächst die Aufmerksamkeit. Daran anschließend werden die als typisch für Erzählungen über Extremereignisse zu beurteilenden szenisch-episodischen Darstellungen mit ihrem besonderen Merkmal der direkten Rede untersucht. Als wichtige Bestandteile der Erzählmuster können zudem spezifische Topoi des Erzählens herausgearbeitet werden.

der mentalen Assoziation zugänglich. "Volmari, Christian: Vernakulare Fotografie zwischen analogen und digitalen Gebrauchsweisen. Göttingen 2012, S. 40.

427 Vgl. Pagenstecher, Cord: Zwischen Tourismuswerbung und Autobiografie. Erzählkulturen in Urlaubsalben. In: Spode, Hasso/Ziehe, Irene (Hg.): Gebuchte Gefühle. Tourismus zwischen Verortung und Entgrenzung. München/Wien 2005, S. 82-91, S. 84.

429 Zur Transdisziplinarität siehe Kapitel 1.2.

430 „Was ich mehrmals durchdacht und erzählt habe, bereitet meiner Erinnerung keine großen Schwierigkeiten mehr. Und mehrfach Erzähltes ergibt in der Situation dann auch eine ,flüssige Geschichte، [...] Auf dem Wege der Verfestigung ergeben sich dann für den Erzählenden eingefahrene, wohlvertraute Leitlinien seines Erzählens." Lehmann 1983, S, 23. 


\section{Leitlinien}

Eine große Auffälligkeit in den erhobenen Interviews ist die Ähnlichkeit der Ereignisschilderungen mit schematischen Darstellungsweisen aus dem Fundus der in der Gesellschaft verankerten Meistererzählung. ${ }^{431}$ Nahezu alle fünfzig Befragten scheinen ihre persönlichen Masternarrationen daran ausgerichtet zu haben. Zwar wird aus der Besonderheit der eigenen Situation heraus argumentiert, aber in diesen als „Schlüsselerzählungen “ 432 wird sowohl auf jene inhaltlichen Vorbilder zurückgegriffen als auch auf strukturelle Vorbilder.

Die Befragten bedienen sich eines „Emplotments“, also einer sequenziellen Struktur, die den Ablauf der Geschehnisse mit Bedeutungen versieht und nachvollziehbar macht. ${ }^{433} \mathrm{Als}$ linear konzipierte Erzählungen folgen sie einer formalisierten Darstellung in Anlehnung an das Drama: neben einer Plotstruktur mit einer Exposition des Themas und einem plausiblen Ende, einer Spannungssteigerung (Suspense) und Wendepunkten, Haupt- und Nebenfiguren sowie situiert in einem Setting, das Orte und Milieus, Sachverhalten und Lebensumstände, andere Menschen und verlorene Objekte zum Inhalt hat. Dieses Setting wird, wie Lucius-Hoene und Deppermann hervorheben, „benötigt, um den Hergang der Ereignisse, aber auch die spezifischen evaluativen Botschaften der Erzählung plausibel zu machen und Bedingungsfaktoren einzufügen. “434

Die von den Befragten verwendeten, thematischen Leitlinien orientieren sich in der Schilderung des Hergangs an einem ,roten Faden“, dem „Ordnungsprinzip“435 der Chronologie. Die Zeitzeug_innen beginnen ihre Erinnerungserzählungen mit Beschreibungen des Alltags innerhalb ihres sozialräumlichen Nahbereichs, konkret am Freitag, den 16. Februar 1962, also an dem Tag, bevor nachts die Deiche brachen. ${ }^{436}$ Darin sind zumeist Beschreibungen der Witterungsverhältnisse integriert.

Der erste erzählerische Wendepunkt ist der Moment, in dem die Befragten der Gefahr durch das Wasser gewahr wurden. Sehr detailreiche Schilderungen, im Sinne einer szenisch-episodischen Darstellung, zum Teil mit anekdotischem Charakter sowie unter Verwendung direkter Rede, werden von allen Befragten nun vermehrt genutzt: die Maßnahmen, die getroffen wurden - Flucht, Ausharren, Versuche der Rettung von materiellen Gütern - sowie insgesamt die Erlebnisse während der Nacht bilden den Kern der Ausführungen. Sie enden mit Schilderungen der Eindrücke des nächsten Morgens, an dem bei Tagesanbruch die Überschwemmungen sichtbar wurden.

431 Ausführlich zur in der Gesellschaft verankerten Meistererzählung siehe Kapitel 2.1.

432 Zum Begriff, „Schlüsselerzählung“ siehe Lucius-Hoene/Deppermann 2004, S. 33.

433 Vgl. White 1991, S. 21ff. Ausführlich hierzu siehe Kapitel 2.5.

434 Lucius-Hoene/Deppermann 2004, S. 63.

435 Jureit 1999, S. 88f. Jureit führt aus: „Die Chronologie gehört als Muster zu den ritualisierten Formen des biographischen Erzählens. “Ebd., S. 89.

436 „All narratives contained an initial segment on where the individual was or what activity he/she was engaged in, followed by an account of observations, thoughts and actions“. Bendix 1990, S. 340 . 
Den zweiten erzählerischen Wendepunkt bildet die jeweilige Rettung, die in den persönlichen Masternarrationen häufig durch die Bundeswehr erfolgte. Diese Schilderungen haben häufig ebenfalls einen anekdotischen Charakter, oder sie weisen einen erklärenden, das Erlebte belegenden Duktus auf. In diesem letzten Teil der Erzählungen, der bereits das Danach der Katastrophe markiert, werden die Ereignisse von nahezu allen Interviewten anhand von bestimmten Schlagwörtern stringent zu einem Ende geführt. Mit je unterschiedlicher Gewichtung sind dies Aspekte von ,Versorgung mit Lebensmitteln', der ,Rückkehr in das Wohnhaus', den dort vorgefundenen ,Schäden und Verlusten' sowie Erläuterungen zum innerfamiliär gemeisterten, Wiederaufbau'. Die Erzählhaltungen münden in einem für die Befragten guten Ausgang der Geschichte.

\section{Direkte Rede}

Eine Besonderheit der durch Leitlinien ausgestalteten persönlichen Masternarrationen ist der Rückgriff auf Modi des szenisch-episodischen Erzählens. Neben anekdotischen Anteilen ist dabei die Verwendung direkter Rede besonders auffällig. In ihrer Wirkung darauf ausgerichtet, sich selbst erzählend als integriert in das memorierte Geschehen darzustellen, erzeugt sie den Eindruck von Unmittelbarkeit und - im Fall der Sturmfluterzählungen - Dramatik. Unterscheiden lassen sich in diesem Zusammenhang Äußerungen, in denen sich die Interviewten selbst zitieren sowie die Wiedergabe von Aussagen anderer, damals anwesender Personen, zumeist älterer Verwandter, die Nebenfiguren in den Erzählungen sind. Ihnen wird ein Wissensvorsprung attestiert, häufig dadurch dass ihre Vorahnungen thematisiert werden.

Hans-Heinrich Hofmann aus Wilhelmsburg, Ortsteil Kirchdorf, schildert:

„Gegen ein Uhr nachts, äh, äh, kam dann, erklang Sirenengeheul. Damals war Feuer noch zwölf Mal Heulton und äh, diese zwölf Mal wurden überschritten und es heulte immer weiter und dann sagte mein Bruder: ,Das ist kein Feuer mehr."

Und Gerhard Wendt, ebenfalls aus Kirchdorf, betont:

„Und mein Großvater sagte damals schon: [...], Leute es ist also, es wird dramatisch, wenn ich das seh, was alles zusammenkommt, äh, was also Wasser in die Elbe bringen kann, Windrichtung und was alles so da drauf, alles da auf uns zukommt, es wird dramatisch."“

Damalige Kinder heben durch die Verwendung direkter Rede die Bedeutung ihrer Eltern hervor. Brigitte Krause aus Wilhelmsburg, Ortsteil Georgswerder, damals 13 Jahre alt, führt aus:

„Und ähm, dann hat unser Vater immer gesagt, ,Also wenn's nun noch höher kommt, müssen wir eben auf die Stühle und auf die Tische und auf die 
Schränke steigen', und es kam ja dann auch so, dass wir alle dann da hoch mussten."

Und auch Maren Schaub aus Neuenfelde, damals 14 Jahre alt, erzählt:

„[...] bis meine Mutter uns raus holte, uns alle anzog und sagte: ,Wir müssen raus'. Und das ging ziemlich hopplahopp. Wir haben also gar nicht so uns auf schick oder fein, sondern warm angezogen. [...] Und mein Vater hat gesagt zu meiner Mutter: ,Du gehst jetzt mit den beiden Mädchen raus, geh in die Kirche."“

Die Verwendung dieser Zitate hat im Interview zudem eine belegende Funktion, wenn der geschilderte Sachverhalt selbst nicht mehr genau erinnert wird, wie etwa

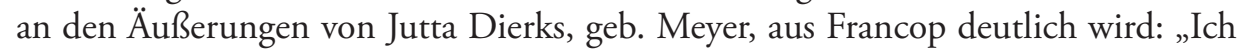
glaub die Idee hatte meine Mutter: ,Lass uns alle in ein Zimmer gehen, wenn irgendwas passiert, denn sind wir alle zusammen."“

Das markanteste Zitat, das von vielen Interviewten in die Erzählungen integriert ist, lautet „Das Wasser kommt!“, zumeist retrospektiv einem Nachbarn ,in den Mund gelegt', von dem die Befragten geweckt wurden. Exemplarisch dafür steht die Aussage von Gerhard Felka aus Wilhelmsburg, Ortsteil Georgswerder: „Da war denn der Nachbar da, und der rief nur, ,Raus, raus, raus, das Wasser kommt '."

Ernst Meyer aus Wilhelmsburg, Ortsteil Georgswerder, nutzt die direkte Rede zur Spannungssteigerung, indem er eine Frage formuliert, die die eigene Hilflosigkeit in den Mittelpunkt stellt: „Dann haben wir in dieser Nacht natürlich gezittert, gemacht, getan, am Fenster gestanden, ,was machen wir bloß, was soll denn nun bloß werden?"."

Damit einher geht die Beschreibung eigener Sprachlosigkeit, wie etwa von Gerhard Felka formuliert: „Man hat nur das Wasser gesehen, nur das Wasser. ,Scheiße hat man gesagt. [...] Da hat man denn auch die Zerstörung gesehen, was das Wasser gemacht hat. Und da konnte man nur mit'm Kopf schütteln, was das Wasser für Macht hat."

Für die Ausgestaltung der persönlichen Masternarrationen ist nicht nur die Einbeziehung direkter Rede konstitutiv und stellt ein wichtiges Unterscheidungsmerkmal zu der in der Gesellschaft verankerten Meistererzählung dar. Auch spezifische Topoi durchziehen die Erinnerungsgeschichten.

\section{Topoi}

Auch wenn für eine Untersuchung von Topoi der Einbezug des autobiografischen Kontextes unerlässlich ist, darf nicht außer Acht gelassen werden, dass diese „Schlüsselsätze persönlicher Erfahrung“ insgesamt „kollektive Erfahrungen widerspiegeln“. 437

437 Schröder 1992, S. 248. Lehmann fasst diese Verknüpfungen folgendermaßen zusammen: „Topoi stehen im Schnittpunkt sozialer und individueller Wertmaßstäbe. Sie sind subjektive Äußerungen und zugleich Indikatoren für kulturelle Normen. Sie sind zur typisierten Erinnerung geronnene persönliche und soziale Erfahrungen. Und sie sind Teile übergreifender kultureller Bild- 
Topoi sind Formulierungen, die in der Regel als wiederkehrendes Motiv in kurzen Einzelsätzen in Erzähltexten zu finden sind. Der Soziologe Hubert Knoblauch definiert Topoi als „feststehende Redeweisen, konstante Motive, verfügbare und stereotype Denkmodelle sowie Klischees. " ${ }^{438}$ Erst ihr wiederholtes Auftreten lässt auf ihre Bedeutung als eine mit bestimmten Wertungen versehene Denkfigur schließen. Im Kontext einer kulturwissenschaftlichen Bewusstseinsanalyse ist dabei weniger der exakte Wortlaut von Interesse, sondern die Wiederholung ihres bewertenden Gehalts. Somit markiert die Ähnlichkeit der Aussagen eine solche Sprachfigur und nicht ihr feststehender Charakter. Oder wie Schröder zusammenfasst: „Mit diesen Festlegungen wird der Topos als eine Sprachfigur aufgefasst, in der sich formale und inhaltliche Merkmale zu einem Amalgam verbinden. “439

Die von ihm im Kontext von Erzählungen ehemaliger deutscher Soldaten über ihre Erfahrungen während des Zweiten Weltkrieges in der Sowjetunion analysierten „Topoi autobiographischen Erzählens" sind auch für die hier untersuchten Interviews erkenntnisleitend. ${ }^{440}$ Schröder spricht zum einen von „Topoi der Beglaubigung“, also von Formulierungen, die den Wahrheitsgehalt der getätigten Äußerung unterstreichen sollen, wie etwa „wenn ich richtig informiert bin“ (Herbert Wenzel), „ich kann mich noch erinnern" (Maren Schaub) oder "das weiß ich" (Helga Rostowski). Diese Aussagen können sowohl als Markierungen der Selbstvergewisserung als auch als „Beglaubigungs- oder auch Beweisformel“ gegenüber der Interviewerin interpretiert werden. ${ }^{441}$ Des Weiteren führt Schröder „Topoi zur Schwierigkeit der Versprachlichung" an, die sich allerdings hier nur sehr gering in Aussprüche, etwa von Adolf Fick („wie soll ich sagen?" und „man kann's nicht beschreiben“) oder von Gerhard Felka („,Scheiße hat man gesagt") zeigen. Diese beziehen sich allesamt auf die Problematik, damals empfundene Gefühlslagen zu verbalisieren und sind Bestandteil des auf eine Bilanzierung der Ereignisse abzielenden Nachfrageteils der Interviews. Im Kontext ihrer persönlichen Masternarrationen hingegen formulieren alle Befragten stringent und souverän ihre Geschichten. Dies interpretiere ich als einen Beleg dafür, dass die Erinnerungen bereits mehrfach ausgetauscht und auf ihre Erzählwürdigkeit hin überprüft wurden, die Geschichten somit verfestigt sind. ${ }^{442}$

komplexe." Lehmann, Albrecht: Der Schicksalsvergleich. Eine Gattung des Erzählens und eine Methode des Erinnerns. In: Bönisch-Brednich, Brigitte/Brednich, Rolf Wilhelm/Gerndt, Helge (Hg.): Erinnern und Vergessen. Vorträge des 27. Deutschen Volkskundekongresses Göttingen 1989. Göttingen 1991, S. 197-207, S. 205.

438 Knoblauch, Hubert: Topik und Soziologie. Von der sozialen und kommunikativen Topik. In: Schirren, Thomas/Ueding, Gert (Hg.): Topik und Rhetorik. Ein interdisziplinäres Symposium. Tübingen 2000, S. 651-667, S. 655.

439 Schröder, Hans Joachim: Topoi des autobiographischen Erzählens. In: Hengartner, Thomas/ Schmidt-Lauber, Brigitta (Hg.): Leben - Erzählen. Beiträge zur Erzähl- und Biographieforschung. Festschrift für Albrecht Lehmann. Berlin/Hamburg 2005, S. 17-42, S. 20.

440 Siehe ausführlich Schröder 1992, S. $227 \mathrm{fff}$. und Schröder 2005, S. $21 \mathrm{ff}$.

441 Vgl. Schröder 2005, S. 23.

442 In diesem Zusammenhang steht der zunächst überraschende Befund, dass der Begriff, Katastrophe so gut wie gar nicht zum erzählerischen Repertoire der Zeitzeug_innen gehört. Siehe ausführlich 
Die auffallend häufigen Bekenntnisse von damaliger Unwissenheit und Ohnmacht, die die persönlichen Masternarrationen durchziehen, interpretiere ich im Zusammenhang mit einer erfolgreichen Bewältigung der Geschehnisse. Diesen Äußerungen haftet nichts ,Peinliches' an, da die Erzählungen allesamt in einer (Wieder-)Erlangung von Wissen und in der Wiederherstellung von Ordnung - hier verstanden als Gegensatz zur beschriebenen Ohnmacht - kulminieren. ${ }^{443}$ Diese, wie ich sie nenne, Topoi der Unkenntnis sind hier die wesentlichste Sprachfigur. Sie wird von allen Altersgruppen gleichermaßen genutzt. Als Beispiele angeführt seien Äußerungen von Maren Schaub (,aber als Kind hab ich ja keine Vorstellung gehabt, was das bedeutet“ und „es war ne Ohnmacht da, ne, man wusste nicht, was man tun sollte, ne, es war alles unheimlich“) sowie „sodass wir überhaupt keine Vorstellung hatten, wo ist das Wasser oder wie weit ist das Wasser“ (Ingrid Kannen), „nun wusste ich ja auch nicht darum, dass so viele Leute gestorben waren oder überhaupt Leute gestorben sind“ (Claus Bode), „,was machen wir bloß, was soll denn nun bloß werden?"“ (Ernst Meyer) und „,was ist los?’ Aber eine Antwort konnten wir uns nicht geben." (Adolf Kröger). Dieser Topos hat also auch einen bekennenden Charakter. Die eigene Unwissenheit wird eingestanden und somit der gesellschaftlich legitimierten Opferrolle durchaus entsprochen, wie sie sich in der öffentlichen Meistererzählung durch die ausschließlich quantifizierende Darstellung - den sogenannten „Opferzahlen“ - zeigt. Wie auch Topaloglou und Tschugguel im Kontext von durch Katastrophen hervorgerufenen Traumatisierungen hervorheben, dominieren zunächst „Gefühle von Hilflosigkeit, Ohnmacht und des Ausgeliefertseins. " ${ }^{444}$ Nach den Ereignissen wurde den Menschen durch staatliche finanzielle Unterstützung ermöglicht, materielle Schäden zu beseitigen, und bereits mit dem aktiv durch die Betroffenen vorangetriebenen Wiederaufbau wurden bisherige Selbstzuschreibungen, also ein Opferstatus, abgemildert. ${ }^{445}$ Ihre Erfolgsgeschichten finden insgesamt bis heute im Austausch untereinander und durch eine mediale Verbreitung der in der Gesellschaft verankerten Meistererzählung Bestätigung.

Diese hier ausgeführten Erzählmuster sind für die persönlichen Masternarrationen konstitutiv. Hinzu kommen inhaltliche Bezüge, die die Befragten in ihren Erlebnisschilderungen herstellen. Eine Analyse der Leitmotive des Erzählens wird daher im Folgenden vertieft.

hierzu Kapitel 4.2.3.

443 Lehmann interpretiert diese Darstellungsweise als „Entlastungsformel zum Selbstschutz gegen die Peinlichkeit subjektiver Gefühlsäußerungen." Lehmann 2007, S. 177.

444 Topaloglou/Tschugguel 2015, S. 40.

445 Vgl. Wörner, Simone: Hilfsprogramme - Maßnahmen für die Betroffenen. In: Hötte, Herbert (Hg.): Die große Flut. Katastrophe - Herausforderung - Perspektiven. Begleitbuch zu den Ausstellungen im Hamburger Rathaus und im Museum für Hamburgische Geschichte aus Anlass des 50. Jahrestages der Sturmflut von 1962. Hamburg 2012, S. 58-65. 


\subsubsection{Leitmotive des Erzählens}

Innerhalb der persönlichen Masternarrationen fungieren Leitmotive als Erzählorientierungen. ${ }^{446}$ Neben sozial-räumlich begründeten Orientierungen werden vielfach Sinneseindrücke hinzugezogen. Leitmotive des Erzählens sind für eine filmische Darstellung besonders geeignet, $\mathrm{da}$ in diesen Interviewaussagen zumeist auf ,innere Bilder' rekurriert wird. Diese können durch das Wissensformat Film thematisiert und vermittelt werden, da es sowohl auf einer auditiven (hier: gesprochene Interviews) als auch auf einer visuellen Ebene (hier: Protagonist_innen; szenisch-episodische Darstellungen; zeitgenössische Fotografien und Filmmaterial) zu argumentierten vermag.

\section{Soziale und räumliche Orientierungen}

Zunächst ist auffällig, dass die Befragten ihre damaligen Erfahrungen aus der Perspektive ihres lokalen und sozialen Umfelds schildern. Während die im kollektiven Gedächtnis der Stadt sowie deutschlandweit verankerte Meistererzählung lokal eher unspezifisch ist und lediglich, bedingt durch die hohen Todeszahlen, der Stadtteil Wilhelmsburg Erwähnung findet, so fokussieren die Befragten in den persönlichen Masternarrationen zumeist sehr dezidiert auf ihre unmittelbare Umgebung. Dies entspricht ihrem damaligen Erfahrungsraum, der in der Regel auf das Wohnhaus und das direkte Umfeld begrenzt war. In den Erzählungen entstehen „Erlebnisräume “447, in denen andere Menschen, Interaktionen und räumliche „Atmosphären" ${ }^{448}$ von Bedeutung sind.

Der Familie kommt in den Erzählungen eine zentrale Bedeutung zu, sie fungiert als wesentlicher Bezugsrahmen für die eigenen Erlebnisschilderungen. Den Abend vor den Deichbrüchen verbrachten nahezu alle Befragten mit ihren Familien zu Hause. Einige verfolgten gemeinsam das Fernsehprogramm oder die Wasserstände an nahegelegenen Deichen. ${ }^{449}$ Anschließend legte man sich schlafen und wurde zumeist von Nachbarn oder Angehörigen geweckt. Als exemplarisch für diese

446 Lehmann unterscheidet anhand seiner Untersuchungsergebnisse zwischen, privaten` Erzählorientierungen, wie Wohnort und beruflichem Hintergrund sowie lebensgeschichtlichen Orientierungen anhand von Erzählungen über die Kindheit bzw. über das Familienleben. Vgl. Lehmann 1983, S. 173-278.

447 Lehmann, Albrecht: Aspekte populären Landschaftsbewußtseins. In: Siemann, Wolfram (Hg.): Umweltgeschichte. Themen und Perspektiven. München 2003, S. 147-164, S. 149.

448 Als Grundlagenwerk gilt: Böhme, Gernot: Atmosphäre. Essays zur neuen Ästhetik. Frankfurt a. M. 1995.

449 Heßler und Kehrt konstatieren in Bezug auf die Sturmflut von 1962, man könne „von einer „Naturvergessenheit' der Hamburger Stadtgesellschaft sprechen“. Heßler/Kehrt 2014, S. 20, mit Bezug auf Altneer, Günter: Naturvergessenheit - Grundlagen einer umfassenden Bioethik. Darmstadt 1991. Diese Untersuchung kommt zu einem differenzierteren Ergebnis. Bestätigt werden kann diese Aussage für Bewohner_innen des städtisch geprägten Wilhelmsburger Ortsteils Reiherstieg sowie in Teilen für zugezogene Behelfsheimsiedler_innen auch anderer Stadtteile. Interviewpartner_innen aus ländlich geprägten Stadt- und Ortsteilen heben hervor, besorgt 
zwei Bezugsrahmen kann die Äußerung von Gerhard Felka angesehen werden, der damals in einer Behelfsheimsiedlung im Wilhelmsburger Ortsteil Georgswerder wohnte:

„Da war denn der Nachbar da, und der rief nur, ,Raus, raus, raus, das Wasser kommt'. Na ja, als Wilhelmsburger, äh, kenn ich so was mit dem Hochwasser und mit der Flut und so was, mir ist so was bekannt. Und, ich hab natürlich da drauf gleich reagiert, ich sag zur Frau, ,Raus, raus`, da war denn aber der Strom schon weg, und denn im Dunkeln, alles raussuchen, anziehen, die Frau wollte Waschen, die an-, die Klamotten waren denn inne Wäsche, und wir haben denn im Dunkeln aus'm Schrank da rausgeholt, was wir grade zu fassen kriegten, zum Anziehen. Und, na ja, wie wir denn raus kamen, der Mond schien taghell, und da hab ich denn schon gesehen, wie ich raus kam, die kleinen Gräben, die waren schon voll Wasser. Und wie ich das gesehen hab, da bin ich losgerast mit der Frau, die hab ich gezogen wie so'n Weltmeister, und denn raus hin zur Schule, dass wir da erstmal höher kamen, in Sicherheit."

Die Menschen waren räumlich durch das Wasser ,festgesetzt', somit gewinnt im Verlauf der Erzählungen die unmittelbare Umgebung an Bedeutung. Informationen über die Gesamtsituation, also über die anderen Stadtteile bzw. selbst darüber, was einige Straßenzüge weiter geschehen war, fehlten und konnten auch nicht ermittelt werden. Da die Stromversorgung nicht mehr funktionierte, wäre dies lediglich über batteriebetriebene Rundfunkgeräte möglich gewesen. Ingrid Kannen aus Wilhelmsburg, Ortsteil Kirchdorf, erinnert sich: „Und haben dann alle Mann am Radio gesessen und versuchten irgendwas zu erfahren. Aber es war eigentlich doch recht spärlich, sodass wir überhaupt keine Vorstellung hatten, wo ist das Wasser oder wie weit ist das Wasser."

Und Adolf Kröger aus Finkenwerder formuliert:

„Und das ganze Ausmaß der Flutkatastrophe haben wir erst in dem Augenblick erfahren, als Feuerwehr, Polizei mit Schlauchbooten, Hubschrauber in der Luft zu sehen waren, dann haben wir gedacht, ,Oh, jetzt muss es aber fürchterlich gewesen sein, was ist los?‘ Aber eine Antwort konnten wir uns nicht geben. Wir konnten uns nur mit dem befassen, was also in Sichtweite, in unserer unmittelbaren Nähe war."

Allerdings differenziert diese „räumliche Dimension“ “450 der Erinnerungen die Erzählungen auch deutlich aus. Dies ist primär darauf zurückzuführen, dass die Befragten nicht nur voneinander Abweichendes an verschiedenen Orten erlebt haben, mehr noch unterscheiden sich die Geschichten aufgrund der damaligen Bedrohungs-

bzw. aufmerksam bezüglich der damaligen Wasserstände gewesen zu sein. Eine „Naturvergessenheit" kann somit nicht grundsätzlich bestätigt werden. 
grade: ob jemand die Nacht auf dem Dach seines Behelfsheimes oder im dritten Stock eines Mietshauses verbrachte, ob man unmittelbar an einer Deichbruchstelle wohnte, wo die Wassermassen hereinbrachen oder im Hinterland, wo das Wasser weit weniger starke Kräfte aufwies, all dies evoziert räumlich prädisponierte Unterschiede in den persönlichen Masternarrationen. In den Interviews ist diese lokale Bezugnahme dennoch eine wesentliche Erzählorientierung.

Für die Zeitzeug_innen, die nach wie vor in den damals überfluteten Stadtteilen wohnen oder private Kontakte dorthin pflegen, sind die Ereignisse darüber hinaus heutzutage aufgrund der Auswirkungen, die die Sturmflut von 1962 auf die Stadtteile hatte „in die Landschaft eingeschrieben. “451 Dies ist sowohl in Bezug auf die verschiedenen Wilhelmsburger Ortsteile - in den Interviews sind dies das Reiherstiegviertel, Georgswerder und Kirchdorf - festzustellen als auch bei Bewohner_innen des ehemaligen Fischerdorfes Finkenwerder sowie in den nach dem Groß-Hamburg-Gesetz 1937 eingemeindeten Dörfern, Altenwerder, Francop und Neuenfelde. Während die beiden letztgenannten Stadtteile nach wie vor einen dörflich geprägten Charakter aufweisen, so ist Altenwerder nur noch als Hafenterminal bekannt und somit heutzutage kaum mehr als eine dörfliche Chiffre. Und auch Waltershof, eine damals belebte Hafen-Siedlung, wird ausschließlich mit den heutigen Kaianlagen in Verbindung gebracht. Ein Hafenerweiterungsgesetz war bereits 1961 in Kraft getreten und hatte damit vor den Ereignissen der Sturmflut Altenwerder, Waltershof sowie Moorburg in den Blick genommen. Diese Stadtteile wurden entweder unmittelbar nach 1962 für unbewohnbar erklärt (Waltershof) oder anschließend sukzessive umstrukturiert bzw. entsiedelt.

Wesentliche Baumaßnahmen als Reaktion auf die Sturmflut waren ab 1962 umfassenden Deichneugestaltungen (Erhöhung und modernere Bauweise der Hochwasserschutzanlagen), der (Neu-)Bau von Sperrwerken und Pumpstationen sowie die Abdeichung der alten Süderelbe in Finkenwerder, was eine Ansiedlung der DASA-Werke (heute Airbus) im Jahr 1970 begünstigte. Auch Planungen zur Umsiedlung von Bevölkerungsteilen des städtisch geprägten Wilhelmsburger Reiherstiegviertels, mit dem Ziel, dort einen Industriestandort zu errichten - was für den Ortsteil Neuhof mit dem Bau der Köhlbrandbrücke letzten Endes durchgeführt wurde - waren projektiert. ${ }^{452}$ Diese Planungen begründen dort stagnierende Investitionen in Modernisierungsvorhaben des Wohnungsbaus bis in die 1990er Jahre und damit einhergehend den Wandel der Bevölkerungsstruktur. ${ }^{453}$ Diese Veränderungen sind den Befragten gegenwärtig und finden trotz des inhaltlichen Fokus in den

$451 \quad$ Fischer 2016, S. 47.

452 Vgl. Schemmer, Janine: Ins Zentrum gerückt. Kultur auf der Elbinsel: Das Bürgerhaus Wilhelmsburg. In: Forschungsstelle für Zeitgeschichte in Hamburg (Hg.): 19 Tage Hamburg.

Ereignisse und Entwicklungen der Stadtgeschichte seit den fünfziger Jahren. München/ Hamburg 2012, S. 249-262, S. 252.

453 Vgl. Markert, Margret: Eine Insel wird Industriegebiet - Portrait des Reiherstiegviertels. In: Geschichtswerkstatt Wilhelmsburg \& Hafen/Museum Elbinsel Wilhelmsburg (Hg.): Wilhelmsburg. Hamburgs große Elbinsel. Hamburg 2008, S. 41-58, S. 55. 
Interviews auf die Ereignisse von 1962 sehr häufig Erwähnung, insbesondere die Themen ,Ausländerzuzug', ,Elbvertiefung', ,Hochwasserschutz ${ }^{6}$ und ,Airbus' bzw. die geplante Startbahnverlängerung in Neuenfelde. Zum Zeitpunkt der Interviews im Jahr 2004 wurde darüber vor Ort höchst kontrovers innerhalb der dortigen Bevölkerung diskutiert.

Unabhängig davon werden in den persönlichen Masternarrationen zur Sturmflut von 1962 primär genuin soziale Orientierungen durch solidarisierende Charakteristiken deutlich. Die Betonung eines gemeinsamen Erfahrungsraumes erfolgt anhand von ,Wir'-Erzählungen. Die lokalen Gegebenheiten werden nicht nur kleinteilig beschrieben, wie oben dargelegt, zudem stellt man sich gewissermaßen andere Personen, zumeist Familienmitglieder oder Nachbarn, erzählerisch an die Seite. Je nach Lebensalter, Geschlecht und damaliger konkreter Gefährdungssituation kann dieser narrativ gestaltete Raum mit Implikationen von Verantwortung, Schutz oder Schicksal konnotiert sein. ${ }^{454}$ Unabhängig davon, ob sich der bzw. die Erzähler_in als handelnde Person und somit als Inhaber_in von Kontrollmöglichkeiten und Entscheidungsspielräumen darstellt oder als Opfer „kontingenter Ereignisse“, die Geschichten sind stets in soziale Situationen eingebunden. ${ }^{455}$

Dennoch haben die Befragten sich ihre je individuellen Erzählperspektiven und Schwerpunktsetzungen bewahrt, was besonders in persönlichen Masternarrationen deutlich wird, die sich auf gemeinsame Erlebnisse gründen. Der sich hierbei ergänzende Charakter der Schilderungen wird im Film am Beispiel der Aussagen der Schwestern Brigitte Krause und Helga Rostowski, geb. Krause, verdeutlicht, die zusammen mit ihren Eltern, weiteren Geschwistern und Nachbar_innen die Nacht in einer Behelfsheimsiedlung ausharren mussten:

Brigitte Krause: „Das Wasser ging nachher so, dass dreiviertel vom Haus war schon unter Wasser, ne. Also die Fenster waren vielleicht noch einen Meter frei sozusagen, ne. [...] Und ähm, dann hat unser Vater immer gesagt, ,Also wenn's nun noch höher kommt, müssen wir eben auf die Stühle und auf die Tische und auf die Schränke steigen'. Und es kam ja dann auch so, dass wir alle dann da hoch mussten."

Helga Rostowski: „Ich weiß, dass meine Mutter gegenüber, die stand an der Seite der Wand, die hat ne Weile auf'n Nachtschrank gestanden, der ist dann aber als er eben ganz unter Wasser war zusammengebrochen, und da hat man versucht, so eine Leiter, so eine Hausleiter so wohl, da hat sie dann draufgestanden ne Weile. Und dann waren in dem Haus noch Nachbarn, die neben uns wohnten, zwei ältere Leute, die standen einer auf'n kleinen Schrank und der Mann stand auf dem Ofen, und als bei dem Mann das Wasser wohl so

\footnotetext{
$454 \mathrm{Zu}$ geschlechterspezifischen Besonderheiten im Erzählen über die damaligen Erfahrungen siehe ausführlich Paech 2013.

455 Vgl. Lucius-Hoene/Deppermann 2004, S. 59.
} 
hoch war, dass das bis zum Herzen, ist er jedenfalls umgekippt, der ist also gestorben da vor uns."

Brigitte Krause erwähnt die Nachbar_innen nicht, sondern sie führt aus beobachtender Erzählperspektive weiter aus:

„Und ähm, irgendwann hat der Vater versucht, durch dieses Dach, was da vorhanden war, das war so mit Dachpappe, äh, keine Dachpfannen, wie man das heute so kennt, eben mit Dachpappe, da hat er versucht, von innen irgendwie ein Loch da hoch zu, kaputt zu machen, und äh, sich da durchzuwringen, aber das war ne stundenlange Arbeit, und er bekam eben von dieser Dachpappe immer diese, diese Krümel davon ins Auge, weil es ja so stürmisch war, ne. Aber irgendwann hat er das mal geschafft und ähm, hat denn einen Teil, ich weiß nicht, welche Geschwister da hochgezogen."

Helga Rostowski erzählt aus einer primär involvierten Perspektive:

„Und denn sind wir eben, ja, alle einzeln denn so aufs Dach, und, das war ein schräges Dach mit Dachpappe, wie man das ja so früher hatte, und da hat mein Vater uns eben so nach und nach rauf gebracht, ja, und da hatten wir dann so in schräger Stellung sozusagen den Rest der Nacht verbracht."

Dass sich die persönlichen Masternarrationen nicht nur ergänzen, sondern auch durchaus voneinander abweichen können, wird am Beispiel der Familie Meyer aus Francop deutlich. ${ }^{456}$ Da das Wohnhaus in unmittelbarer Nähe zum Deich stand, waren die Familienmitglieder durch die Deichbrüche unmittelbar vom Wasser bedroht. Sie wurden am folgenden Tag per Hubschrauber evakuiert.

Bernd Meyer, damals 12 Jahre alt, schildert:

„Und denn haben wir oben so zwei Zimmer, die waren vermietet, da hat ne ältere Frau gewohnt, die war wie Oma im Haus, und die hatte Zeitungen, und denn sind wir denn bei Tante Emma in der Stube gewesen und hab diese Zeitung geholt aus'n Zeitungsständer und der stand direkt am Fenster. Und bin dann praktisch so drei Meter vom Fenster weg gewesen und da war bloß noch einmal nen Zischen zu hören, ,sch', und da konnten wir ins Freie gucken, da war der ganze Giebel weg."

Seine jüngere Schwester, Gitta Rosenau, damals 8 Jahre alt, erzählt:

„Und da haben wir eben halt gesessen bis es irgendwann einen unheimlichen Knall gegeben hat, und da kam mein Vater hoch, und wir denn immer, Vati, was hat da eben so geknallt?', ,Ach nein, das war nichts', und so. Und irgendwann zog das ganz fürchterlich im Haus, wir hatten oben damals vermietet

456 Dieses Beispiel fand im Film keine Berücksichtigung, jedoch nicht weil die Aussagen voneinander abweichen, sondern da es in das übergeordnete dramaturgische Gesamtkonzept von Flut 1962 Erinnern. Gedenken. Erzählen. nicht integriert werden konnte. Zu den Herausforderungen von Auswahl- und Montageverfahren in Bezug auf gefilmte Interviews siehe Kapitel 3.2.3 und 3.2.4. 
an eine ältere Frau, und die war bei ner Bekannten, also war die Wohnung da oben war frei, dass wir uns da aufhalten konnten, zumindestens in der Küche, und irgendwo zog es dann nachher immer, und da haben wir gesagt, irgendwie zieht es hier", und da sagte er dann, ja, ,ich muss euch jetzt ne Mitteilung machen, wir sitzen nur noch im halben Haus, die eine ganze Giebelwand ist weggebrochen'."

Deren ältere Schwester, die damals 18-jährige Jutta Dierks, beschreibt die damalige Situation folgendermaßen:

„Und dann äh hatte, ich glaub die Idee hatte meine Mutter, ,lass uns alle in ein Zimmer gehen, wenn irgendwas passiert, denn sind wir alle zusammen'. So und denn sind wir auch alle zusammen in dem Elternschlafzimmer, hab'n uns da auf die Betten gesetzt und dann hat das Haus ganz ganz furchtbar geschüttelt, also das war so entsetzlich, das, das kann man heute eigentlich so gar nicht mehr nachvollziehen, man hat so gedacht, das ist es jetzt eigentlich, $\mathrm{da}$, da muss was ganz Schreckliches passieren jetzt, ne? Und denn hat er, hat mein Vati denn nachher erst mal geguckt, und dann hat er mich nachher zur Seite genommen und da hat er gesagt, ,komm mal mit mir'. Und dann sind wir raus gegangen und wir hatten eine Wohnung oben im Obergeschoss vermietet, die war aber nicht da, und in deren Wohnung da war der ganze Giebel weg. Und da sacht er, ,erzähl das aber nicht Mutti, denn dreht die uns hier ab“."

Und die angesprochene Mutter, Elsbeth Meyer, zintegriert in ihre Schilderung auch religiöse Aspekte:

„Und dann lief das Wasser ja schon durch den Keller rein und dann sind wir gleich nachem Boden mit den Kindern, und wie ich denn so saß, auf einmal da poltert das immer so. Da sag' ich zu meinem Mann, ich sag',was ist das bloß?'. „Ach' sagt er, ,ich mag das gar nicht sagen. Der Giebel ist schon weg.' Und denn ja, ich hab mich dazu getröstet, mein Schwiegervater sagte ,wir haben einen doppelten Schornstein, der hält uns hier'. Und wir waren im Kinderzimmer und haben da natürlich geklappert, nich, und denn wenn ich ausm Fenster, konnte ich das Wasser ja erreichen. Wenn denn die ersten Schuhe und, und Stubben und was da so durchkam, ,oh' ich sag', ,jetzt ist Finkenwerder wohl abgesoffen'. Also da begann die Angst. Und da habe ich gesagt ,nee', ich hab von mein'n Vater vom Weltkrieg her einen Brief, einen Himmelsbrief, und das war mein Glaube, ich sag', den hab' ich und der beschützt uns', und das hat uns auch Glück gebracht."

Wie diese zwei Beispiele für die Polyphonie der persönlichen Masternarrationen zeigt, ist nicht nur eine implizite Selbstbezüglichkeit festzustellen, also ein „Ich der Erzählung [...], das ohne explizite Charakterisierung in der Geschichte selbst auf- 
geht ${ }^{“ 457}$, sondern auch Komponenten in den Geschichten, die hier als Ich-Erzählungen bezeichnet werden sollen. Insbesondere szenisch-episodische Darstellungen verweisen auf diese Besonderheit, respektive die besondere Dramatik der eigenen Erfahrung, die im Erzählen re-inszeniert wird. ${ }^{458}$ Diese Erzählperspektiven vermitteln zudem einen Eindruck von damaligen interfamiliären Konstellationen und damit verbundenen Selbstzuschreibungen.

Nichtsdestotrotz sind individualisierende Dimensionen der Erzählungen insbesondere bei denjenigen festzustellen, die 1962 noch Kinder waren. Die damals bei allen Betroffenen vorhandenen Gefühle von Ohnmacht und Schutzbedürftigkeit waren vermutlich bei Kindern am stärksten ausgeprägt. Damals erwachsene Interviewpartner_innen heben hingegen solidarisierende Aspekte hervor, insbesondere dadurch, dass sie in der Retrospektion nicht nur auf das gemeinsam ,geteilte Leid fokussieren, sondern ihre persönlichen Masternarrationen stets zu einem guten Ende führen, also auf die gemeinsame Überwindung von existentieller Bedrohung hin konzipieren. Hier ist eine Übereinstimmung bzw. zumindest eine Bezugnahme auf die in der Gesellschaft verankerte Meistererzählung deutlich erkennbar.

Allen Befragten gemeinsam ist also eine primäre Orientierung an zwischenmenschlichen Beziehungen. Im Gegensatz zur in der Gesellschaft verankerten Meistererzählung wird in diesem Zusammenhang vor allem der familiäre und nachbarschaftliche Zusammenhalt hervorgehoben und weniger die finanzielle Unterstützung durch anonyme Spenden oder staatliche Hilfe. ${ }^{459}$ Insbesondere diese Orientierungen können, im Gegensatz zu den in Kapitel 4.2.1 analysierten Leitlinien des Erzählens, die einen primär überindividuellen Gehalt aufweisen, als „subjektive Leistungen“" angesehen werden. 460

Neben lokalen und sozialen Erzählorientierungen kommt in den erhobenen Interviews Sinneseindrücken eine besondere Bedeutung zu.

\section{Sinneseindrücke}

Schilderungen des ,soziale[n] und [des] atmosphärische[n] Situationskontext[es] “461 gehen zumeist Hand in Hand, da es sich bei den geschilderten Erfahrungen um biografische Schlüsselereignisse handelt. Diese als extrem wahrgenommenen Erlebnisse werden zumeist aufgrund ihrer anhaltenden "sinnlichen Präsenz" erinnert. ${ }^{462}$ Reinhard Koselleck konstatiert, dass körperliche und emotionale Empfindungen gleichermaßen unverändert blieben, insbesondere „der Geruch, der Geschmack, das

\footnotetext{
457 Lucius-Hoene/Deppermann 2004, S. 68.

458 Vgl. ebd.

459 Vgl. Wörner 2012.

460 Vgl. Lehmann 1983, S. 26.

461 Lehmann 2007, S. 71.

462 Koselleck, Reinhard: Glühende Lava, zur Erinnerung geronnen. In: Frankfurter Allgemeine Zeitung vom 6. Mai 1995, o. S., zit. nach Welzer 2000, S. 56.
} 
Geräusch, das Gefühl und das sichtbare Umfeld““ ${ }^{463}$ Letzteres ist somit nicht nur, wie oben dargelegt, eine sozial-räumliche Orientierung im Erzählen, sondern erinnerte Orte sind konnotiert mit sinnlichen Wahrnehmungen. ${ }^{464}$

Die erhobenen Erzählungen thematisieren sowohl diese gehörten als auch die gesehenen Eindrücke. Atmosphärische Beschreibungen bilden daher den Einstieg in die persönlichen Masternarrationen, insbesondere der starke Wind. Die Familienväter Herbert Wenzel aus Kirchdorf und Adolf Kröger aus Finkenwerder erinnern sich: „Wir hatten tagelang vorher heftige Weststürme, die hier also gewaltig rüberrauschten [...].“(Wenzel) und: „Der Wind brauste auf, das Wasser lief auf, die Wasser kamen immer höher, und irgendwo im Hinterkopf hat man doch schon das Gefahrenmoment gehabt, erst dann." Adolf Kröger verweist damit bereits auf seinen heutigen Wissenshorizont, nämlich den des drohenden Unheils. Diese Erfahrung von existenzieller Gefährdung begünstigt die Erinnerungsleistung, da, mit Assmann formuliert, „der Affekt der wichtigste Verstärker von Erinnerung ist“ ".465 Und auch Lehmann führt aus: „Die Atmosphäre bleibt in Erinnerung, sie hält die Wirkung eines Ereignisses für die spätere Lebensgeschichte fest. “466

Die Erzählungen über die Ereignisse in der Nacht und insbesondere die Dramatik der eigenen Erlebnisse erhalten eine emotionale Färbung insbesondere dadurch, dass auf akustische Eindrücke fokussiert wird. Vermutlich ist diese Gewichtung davon beeinflusst, dass es in jener Nacht kaum etwas zu sehen gab, da mit dem Strom auch die Beleuchtung ausgefallen war. In Erinnerung bleiben insbesondere das „Sirenengeheul“ und die Warnrufe von Nachbarn sowie das ,Scheppern eines Deichbruchs', "Todesschreie“ der Tiere oder „Hilferufe“ von Nachbar_innen, die sich auf ihr Hausdach geflüchtet hatten. Nichtsdestotrotz sind diese akustischen Eindrücke eng verbunden mit anderen Sinneswahrnehmungen, wie beispielhaft Jutta Dierks aus Francop über ihre Todesangst während des nächtlichen Ausharrens im Wohnhaus deutlich macht:

„So und denn sind wir auch alle zusammen in dem Elternschlafzimmer, hab'n uns da auf die Betten gesetzt und dann hat das Haus ganz ganz furchtbar geschüttelt, also das war so entsetzlich, das, das kann man heute eigentlich so gar nicht mehr nachvollziehen, man hat so gedacht, das ist es jetzt eigentlich."

Optische Eindrücke prägen vorrangig die Schilderungen des nächsten Morgens, des 17. Februars 1962. Insbesondere wird die durch die Wassermassen veränderte Landschaft thematisiert. Ernst Meyer aus Kirchdorf, Ortsteil Georgswerder, beschreibt diese Situation:

„Dann haben wir in dieser Nacht natürlich gezittert, gemacht, getan, am Fenster gestanden, ,was machen wir bloß, was soll denn nun bloß werden?’ Und erst als der Morgen so'n bisschen dämmerte, wurde uns dieses ganze

\footnotetext{
463 Ebd.

464 Zur räumlichen Situiertheit von Eindrücken siehe Böhme 1995.

465 Vgl. Assmann 2003, S. 46.

466 Lehmann 2007, S. 72.
} 
Ausmaß dieser Katastrophe zu bewusst, zum Bewusstsein. Wir guckten auf die gegenüberliegenden Wiesen und sahen eben nur soweit das Auge reichte, Wasser, Wasser, Wasser."

Und Elisabeth Schwartau aus Altenwerder führt aus:

„Soweit man gucken konnte, soweit man links und rechts und wenn man hinten aus dem Fenster guckte, es war alles Wasser, wenn man vorne aus dem Fenster guckte, es war alles Wasser, wo man guckte, es war Wasser. Wasser, Wasser, Wasser."

Die persönlichen Masternarrationen über die Hamburger Sturmflut von 1962 sind durchgängig mit Sinneseindrücken unterlegt. Als optische Eindrücke sind dies vor allem die Wassermassen sowie häufig die umfangreichen Rettungsaktionen per Hubschrauber. So etwas hatten die Menschen vorher noch nicht mit eigenen Augen gesehen. Und auch die akustischen Eindrücke sind an das Besondere, an das zuvor nicht für möglich Gehaltene und auch emotional Berührende gekoppelt, insbesondere - wie oben dargelegt - an die Geräusche während der Nacht.

Darüber hinaus werden auch heutige Haltungen mit damaligen Sinneswahrnehmungen in Verbindung gebracht bzw. begründet. Helga Rostowski aus Wilhelmsburg, Ortsteil Georgswerder, stellt heraus: „Ich wüsste ja jetzt viel eher, was ich machen würde und äh, wäre viel hellhöriger auf alles." Und Marlies Wenzel aus Wilhelmsburg, Ortsteil Kirchdorf, äußert: „Wenn ich denn im Radio höre, der Wind soll noch rumgehen auf Nord-West, also ich schlaf dann die ganze Nacht nicht. Ich bleib die ganze Nacht dann wach." Ähnlich formuliert auch Elisabeth Schwartau aus Altenwerder: „Und wenn so ein, ein Sturm ist jetzt, dann wird man unruhig." Auch Ursula Seemann aus Wilhelmsburg, Ortsteil Reiherstieg, stellt sich die Frage: „Wann kommt das Nächste, wann kommt die nächste Flut? Und bei jedem, äh, ja bei jedem stärkeren Wind, was auch in den Orkan rein ging, hatte man ja Angst." Die erinnerten Sinneseindrücke haben somit großen Anteil an einer Sensibilisierung für heutige Gefahren und Risiken. Dies ist ein für die Argumentation dieser Untersuchung zentraler Befund, der in Kapitel 4.3 weiter ausgeführt wird.

Eine weitere Auffälligkeit in den persönlichen Masternarrationen besteht zudem darin, dass sich die Befragten zwar in ihren Geschichten an der Struktur und den Inhalten der in der Gesellschaft verankerten Meistererzählung orientieren, einige wesentliche Bestandteile werden von ihnen jedoch ausgelassen. Im Folgenden wird diese Besonderheit, die ich als Leerstellen des Erzählens bezeichne, analysiert.

\subsubsection{Leerstellen des Erzählens}

Die Zeitzeug_innen gestalten ihre Erzählungen mit Hilfe von sozial-räumlichen Orientierungen und dezidierten Schilderungen von Sinneseindrücken sowie anhand struktureller und inhaltlicher Bestandteile der in der Gesellschaft verankerten Meistererzählung. Eine weitere Auffälligkeit besteht in Auslassungen von Inhal- 
ten und in Abweichungen von dieser Meistererzählung: zum einen wird der Begriff ,Katastrophe in den Interviews nur äußerst selten verwendet, zum anderen ist die „Kristallisationsgestalt“ ${ }^{467}$ der Hamburger Sturmflut von 1962, der spätere Bundeskanzler Helmut Schmidt, kein Bestandteil der persönlichen Masternarrationen. Im Flut 1962 - Erinnern. Gedenken. Erzählen. wird dem entsprochen, indem diese beiden Komponenten der überindividuellen Meistererzählung zwar integriert, jedoch filmisch als randständig bzw. als zeitgeschichtliches, präfiguriertes Phänomen dargestellt werden: Der Begriff ,Katastrophe' findet lediglich im Vorspann Verwendung, dadurch dass er von einer Zeitzeugin aufgegriffen wird. Hierbei fungiert er als Marker, um das Publikum in die filmische Erzählung einzuführen und mittels dieser ihnen bekannten Vokabel ,abzuholen“. Mit Mauch formuliert, wird er „zur Beschreibung von Ereignissen herangezogen, die sich mit Worten eigentlich nicht mehr erklären lassen, aber dennoch durch sie bestimmt werden. “468 Da die damaligen Ereignisse von den Befragten jedoch ausführlich thematisiert werden, verliert der Terminus hier seine Relevanz, wie noch ausgeführt wird.

Helmut Schmidt wird im zweiten Teil des Films, der die persönlichen Masternarrationen zum Inhalt hat, dramaturgisch integriert. Dies geschieht mit Hilfe von filmischem Archivmaterial, welches in seiner Wirkung darauf ausgerichtet ist, ihn als zeitgenössische Figur zu ,verkapseln‘.

\section{,Katastrophe}

Der Begriff ,Katastrophe ist unspezifisch, oder wie die Soziologen Leon Hempel und Thomas Markwart konstatieren, verfügt er über eine breit konnotierte, insgesamt „politisch-theatralisch-ästhetisch-performative Dimension“"469. Während er in den zeitgenössischen Medien und behördlichen Verlautbarungen sowie in heutigen Publikationen in Zusammenhang mit der Sturmflut von 1962 durchgängig exponiert auftaucht, so ist dies in den Interviews nur in sehr geringem Maße der Fall. Zum Zeitpunkt der Erhebung erscheinen die Lebensverhältnisse der Befragten stabil. Aus dem damaligen Chaos ist längst nicht nur persönlich und lokal, sondern eben auch erzählend eine stabile Ordnung erwachsen. ${ }^{470}$ In den Geschichten hat der Ausdruck ,Katastrophe“ als Sinnbild für das Unbeschreibbare somit mittlerweile ausgedient. Mehr noch wird die damalige „Subjektivität und Unsicherheit“, die mit der Verwendung des Begriffes einher ginge, in den Interviews nicht verschleiert, sondern offensiv thematisiert und reflektiert, wie anhand der Topoi der Unkenntnis bereits ausgeführt wurde. ${ }^{41}$ Die Interviewten artikulieren aus heutiger Perspektive präzise ihre limitierten Handlungsspielräume.

\footnotetext{
467 Zum Terminus „Kristallisationsgestalt“ im Kontext von „politischen Mythologien“ siehe ausführlich Lehmann 2007, S. 129ff.

468 Mauch 2014, S. 132.

469 Hempel/Markwart 2013, S. 11.

470 Ausführlich hierzu siehe Kapitel 4.2.4.

471 Vgl. Hempel/Markwart 2013, S. 14. Siehe hierzu ausführlich Kapitel 4.2.1.
} 
Adolf Fick aus Finkenwerder führt aus: „Und da ist denn auch eine Kuh stecken geblieben, und dann sind natürlich diese, diese Todesschreie, das ist natürlich immer fürchterlich, nich. Und als Kind, äh, ist das natürlich, wenn man dann nicht helfen kann."

Hans-Heinrich Hofmann aus Wilhelmsburg, Ortsteil Kirchdorf, erzählt:

„Auf der gegenüberliegenden Straßenseite, ganz hinten, waren sogenannte Behelfsheime, sogenannte Plattenhäuser, und von dort her klangen Hilferufe, Sie müssen sich vorstellen, Windstärke zehn, elf in Böen zwölf, es klapperte, schepperte, heult, pfeift und dann kommt in Fetzen Hilferufe, richtige Hilferufe, also nicht irgendwie so, ,Hilfe, der will mir was tun', sondern das war richtig Angst, die da auch vermittelt wurde. Ja das ist das, was, äh, eigentlich so an, an gehörten Eindrücken, ja, bis heute geblieben ist. Und das vor dem Hintergrund, da kann man nicht hin, man kann nicht helfen, man kann doch nicht rüber rufen: ,Wir kommen gleich!' Erstens hätt’s keiner gehört, zweitens war's ja auch nicht wahr. Das ist auch 'n schlechtes Gefühl."

Inzwischen - mit zeitlichem Abstand - vermögen es die damals Betroffenen, ihre Erlebnisse nicht nur strukturiert und kohärent zu verbalisieren, sondern auch eigene Gefühle und Deutungen zu thematisieren. Gleichwohl dienen heutige Berichterstattungen über aktuelle, als ,Naturkatastrophen' bezeichnete Ereignisse als Referenz dafür, die eigenen Erlebnisse, mit den Worten der Politikwissenschaftlerin Marie Bartels, „auf der Skala der Entsetzlichkeit [...] zu bewerten als ein Ereignis, das als solches bereits in der Geschichte der Katastrophen Anerkennung gefunden hat. “472

Die im Rahmen dieser Untersuchung interviewten Zeitzeug_innen der Hamburger Sturmflut von 1962 setzen ihre Bewertung des Vergangenen primär jedoch in Beziehung zur Gegenwart. Die latente Gefahr im heutigen stabilen und überschaubaren Leben benennt in diesem Zusammenhang Gerhard Wendt aus Wilhelmsburg, Ortsteil Kirchdorf, einem Stadtteil der unter Normal Null (NN), also unter dem Meeresspiegel, liegt und daher ohne Hochwasserschutzanlagen bei jeder Flut unter Wasser stünde, wenn er äußert:

„Also ich meine, dass das unbedingt erwähnenswert ist und erinnerungswichtig ist, dass wir also diese Sachen auch unseren Kindern und Enkelkindern immer wieder vor Augen führen. Ob das kleine Ereignisse sind wie die Flut, für uns groß, aber für die Weltgeschichte kleine Ereignisse sind, die überall jeden Tag passieren. Aber die unmittelbar hier wohnen und äh, äh, damit leben und dieses Gebiet auch toll finden, dass wir auch sagen: ,Leute, die Gefahr ist da, und die ist überall."“

472 Bartels, Marie: Katastrophen und Kausalität. In: Hempel, Leon/Dies./Markwart, Thomas (Hg.): Aufbruch ins Unversicherbare. Zum Katastrophendiskurs der Gegenwart. Bielefeld 2013, S. 193-207, S. 196. 
In der zeitgenössischen medialen Berichterstattung wurde durch die Einordnung der Ereignisse als ,Katastrophe ' ein Deutungsangebot geschaffen, um eine gesamtgesellschaftlich solidarisierende Haltung zu befördern. ${ }^{473}$ Gleichzeitig führte diese Bewertung des Ereignisses als ,Katastrophe ' dazu, auf Wiedererlangung von Ordnung hin ausgerichtete Handlungsstrategien in Kraft zu setzen. Diese Deutung manifestierte rasch eine Darstellung, die „die ordnende Funktion der administrativen Macht “474 als Antwort mit einschrieb. Die Bundeswehr und weitere Institutionen - Polizei, Feuerwehr, technische Hilfsdienste sowie als Einzelperson der damalige Polizeisenator Helmut Schmidt - wurden so als Ordnung wiederherstellende Akteur_innen implementiert. Dies beinhaltet auch eine Unterscheidung zwischen der Bevölkerung als passive, Hilfe empfangene Opfer einerseits und Vertreter von Hilfsorganisationen als aktive Retter andererseits. Da diese Darstellung in der Meistererzählung bis heute konstitutiv ist, so ist darin den Befragten nach wie vor eine passive Rolle zugewiesen. In den erhobenen Interviews hingegen findet die Rekonstruktion einer Emanzipation der „Katastrophenobjekte“ hin zu agierenden Subjekten statt. ${ }^{475}$

Grundsätzlich hatte die Bezeichnung ,Katastrophe“ also zunächst das Angebot der Zugehörigkeit zu einer „Betroffenengemeinschaft auf Zeit“ geschaffen. ${ }^{476}$ Die humanitäre Helferin in Katastrophengebieten Ina Blümel konstatiert aus ihrer Praxiserfahrung eine „geradezu mythische [...] Erfahrung von Unterschiedslosigkeit“. Zugleich hebt auch Blümel die „implizite hierarchische Struktur“ hervor, die dem Begriff innewohnt. ${ }^{477}$ Wer also ein Extremereignis als ,Katastrophe' behauptet, verschafft sich Handlungsräume, in denen außergewöhnlich agiert werden darf, etwa dadurch, den regionalen Radius für Evakuierungszonen und Hilfsmaßnahmen zu definieren. ${ }^{478}$

473 Vgl. Schott 2002, S. 197

474 Hempel/Markwart 2013, S. 15.

475 Siehe hierzu Kapitel 4.3. Dombrowsky folgert, so fassen Hempel und Markwart zusammen, dass „Katastrophenerklärungen vor dem Hintergrund mündiger Bürger [fraglich werden], die sich in der Ausnahmesituation selbst zu helfen und zu organisieren wissen." Hempel/Markwart 2013,

S. 18, mit Bezug auf Dombrowsky, Wolf R.: Der Mensch als Risiko - oder geht alle Gefahr vom Volke aus? In: Hempel, Leon/Bartels, Marie/Markwart, Thomas (Hg.): Aufbruch ins Unversicherbare. Zum Katastrophendiskurs der Gegenwart. Bielefeld 2013, S. 29-52.

476 Vgl. Keller, Reiner: Zur Chronik angekündigter Katastrophen. Die Umweltkrise als Dauerevent. In: Gebhardt, Winfried/Hitzler, Ronald/Pfadenhauer, Michaela (Hg.): Events. Soziologie des Außergewöhnlichen. Opladen 2000, S. 263-285, S. 280.

477 Vgl. Hempel/Markwart 2013, S. 24, mit Bezug auf Blümel, Ina: „Es lohnt immer, den ganzen Menschen wahrzunehmen. "Ein Interview zur Praxis der humanitären Hilfe. In: Hempel, Leon/ Bartels, Marie/Markwart, Thomas (Hg.): Aufbruch ins Unversicherbare. Zum Katastrophendiskurs der Gegenwart. Bielefeld 2013, S. 421-436.

478 Bundeswehreinsätze sind heutzutage ,ausschließlich im Katastrophenfall erlaubt, worin deutlich wird, dass der Begriff Handlungsräume schafft, die sonst nicht toleriert würden. "Bartels 2013, S. 194. Das nach wie vor bekannteste Beispiel hierfür ist die vom damaligen Polizeisenator Helmut Schmidt initiierte, rechtlich fragwürdige Einbindung der Bundeswehr in die Rettungs- und Bergungsaktionen im Februar 1962. Vgl. Hempel/Markwart 2013, S. 12. Die Autoren merken 
In Flut 1962 - Erinnern. Gedenken. Erzählen. werden anhand von zeitgenössischem Filmmaterial die durch die Bundeswehr erfolgten Rettungs- und Bergungsarbeiten gezeigt. Sowohl in der Meistererzählung als auch in den persönlichen Masternarrationen bilden diese Maßnahmen das ,Danach der Katastrophec, sie sind der erzählerische Wendepunkt, an dem begonnen wurde, das Chaos in eine Ordnung zu transformieren. ${ }^{479}$ Während in den persönlichen Geschichten die von den Betroffenen selbst geleisteten Wiederaufbauarbeiten im Mittelpunkt stehen, so wird dies in der in der Gesellschaft verankerten Meistererzählung den bürokratischen Institutionen, unter Führung des damaligen Polizeisenators Helmut Schmidt, zugeschrieben.

\section{,Helmut Schmidt}

Während in der Meistererzählung der Person Helmut Schmidt eine zentrale Rolle als Krisenmanager zukommt - er kann grundsätzlich als eine der „Kristallisationsfiguren des politischen Erzählens im 20. Jahrhundert “480 angesehen werden - so ist augenfällig, dass er zunächst in den persönlichen Masternarrationen keine Erwähnung findet. Erst auf meine Nachfrage erfolgen standardisiert wirkende, zum Teil floskelhaft anmutende Äußerungen, die erstens auf seine Tatkraft als „Organisator“, so ein Befragter, zweitens auf eine, in Zusammenhang damit stehende, fragliche Gesetzeskonformität seiner Handlungen sowie drittens auf eine große Dankbarkeit ihm gegenüber rekurrieren.

Helmut Schmidt werden von den Zeitzeug_innen bestimmte Charaktereigenschaften zugeschrieben, nämlich aufgrund seiner „Verbindungen“ zur Bundeswehr und zur Nato die "Fäden in der Hand gehabt" und „die Koordinierung an sich genommen " bzw. ,an sich gerissen" und "forciert" zu haben. Seine Initiative wird als „,engagiertes Eingreifen“ gewürdigt, insbesondere wenn betont wird, er habe „die richtigen Entscheidungen zum richtigen Zeitpunkt" getroffen. Dieses, die Situation „im Griff gehabt“ zu haben, wird in Verbindung damit erzählt, dass er die Nerven behielt, „der Mann, der hat Format“. Seine Popularität ist nach wie vor deutlich, wie Edeltraud Schulz aus Wilhelmsburg hervorhebt:

„Also, er hat das echt, ich find den, ich find ihn nach wie vor find ich ihn also einmalig. Muss ich ganz ehrlich sagen, was er geleistet hat. Und ja auch teilweise heute noch, ne. Also, das ist wirklich eine Persönlichkeit, die, da kommt so schnell keiner mit. ${ }^{*}{ }^{81}$

weiter an, dass Schmidt „gewissermaßen das Urteil des Bundesverfassungsgerichts aus dem Jahr 2012 über den Einsatz der Bundeswehr im Katastrophenfall vorweg" nahm. Vgl. ebd.

479 Vgl. Bartels 2013, S. 199 f.

480 Lehmann 2007, S. 130.

481 Diese Äußerung stammt aus einem per Audiogerät aufgezeichneten Interview und fand im Film keine Verwendung. Zu den Auswahlkriterien siehe Kapitel 3.2.3. 
Die von Schmidt erbetene Unterstützung durch die Bundeswehr wurde von ihm Jahre später selbst in seiner Rechtmäßigkeit angezweifelt. ${ }^{482}$ In jenen Tagen im Februar 1962 hatte er sich auf den „übergesetzlichen Notstand“483 berufen. Dies kollidierte mit Artikel 143 des Grundgesetzes. Erst seit der Verabschiedung der Notstandsgesetze im Jahr 1968 ist es verfassungsrechtlich zulässig, dass u. a. auch die Bundeswehr im Falle einer „Naturkatastrophe“ oder eines „Unglücksfalles“ innerhalb des Landes eingesetzt werden kann. ${ }^{484}$

Von den Befragten wird dieses juristisch fragwürdige Verhalten ausnahmslos gutgeheißen. Schmidt habe „sich über alle Kompetenzen“ hinweggesetzt und wie ein Interviewpartner hinzufügt, „da wurde dann ja auch nicht nach Kosten gefragt“. Diese Äußerung bezieht sich darauf, dass auch die unmittelbaren finanziellen Hilfen an die Bevölkerung „aus Gründen des Haushaltrechts“ nicht ohne Weiteres hätten zugesagt bzw. ausgezahlt werden dürfen. ${ }^{485}$ Schmidt wird dies als in der Situation notwendiges Verhalten zugestanden. Christa Henke aus Wilhelmsburg führt aus: „Muss jemand sein, der eben durchgreift und das auf seine Kappe nimmt, Verantwortung zeigt, ne.“

Viele der Befragten bekunden demgemäß eine nach wie vor große Dankbarkeit, die sie Helmut Schmidt bis heute entgegenbringen. Interessant ist jedoch, dass unumwunden eingestanden wird, erst „im Nachhinein“ - zumeist werden hier „die Medien“ als Informationsquelle genannt - von seiner Rolle als „Herr der Flut“486, wie die Zeitschrift DER SPIEGEL titelte, erfahren zu haben. Bereits tagsüber am 16. Februar 1962 und während der darauffolgenden Nacht zum 17. Februar 1962, also bevor Schmidt in der Polizeibehörde am Karl-Muck-Platz eintraf und die Koordination und Leitung der Hilfsmaßnahmen übernahm, hatte es „eine improvisierte Zusammenarbeit der Behörden " 487 sowie von Seiten der Bundeswehr initiierte Hilfsmaßnahmen zur Deichsicherung gegeben.

Hans Peter Göhrke, der 1962 als Wehrdienstleistender in der Kaserne HamburgHeimfeld stationiert war, erläutert:

„Aber am späten Nachmittag (des 16. Februars) rückten wir dann aus und marschierten Richtung Francop. Das ist im Alten Land und dort sollten wir Deiche sichern. Dort hieß es erst einmal das Wasser würde sehr hoch kommen, aber hier ist eine Schwachstelle und das Alte Land befürchtete, dass dort Überschwemmungen sein könnten. Naja, und da haben wir dann Sandsäcke geschleppt und alles Mögliche aufgebaut und Barrieren aufgerichtet bis Mitternacht."

\footnotetext{
482 Vgl. Soell, Helmut: Helmut Schmidt. 1918-1969. Vernunft und Leidenschaft. München 2003, S. 388.

483 Helmut Schmidt im Interview mit dem NDR am 17.02.1962, zit. nach Soell 2003, S. 388.

484 Vgl. Artikel 35 BGBL, Siebzehntes Gesetz zur Ergänzung des Grundgesetzes („Notstandsgesetze“), 24.06.1968, S. 709, zit. nach Mauch 2015, S. 141.

485 Vgl. Soell 2003, S. 388.

486 DER SPIEGEL, 07.03.1962.

487 Soell 2003, S. 391.
} 
Der Historiker Jens Ivo Engels hebt hervor, dass es „wohl naiv“ wäre „anzunehmen, Schmidt verdanke seine Popularität allein der Tatsache, daß er am richtigen Ort zur rechten Zeit das Notwendige tat. “488 Hinzu kam zum einen, dass sich sein „Habitus des Katastrophenmanagers " deutlich von den anderen Politikern unterschied, die allesamt primär durch moralische Argumentationen auffielen denn als Pragmatiker. Nicht Zivilisationskritik, sondern Berichte über Verluste und Koordinierungsmaßnahmen standen bei Schmidt im Mittelpunkt. Engels fasst mit Bezug auf damalige religiöse Deutungen der Geschehnisse zusammen: „Er war nicht Prediger, sondern Techniker. “489

Zum anderen ist es auch Schmidt selbst, der sich durch diese ,Katastrophen'-Erzählung bzw. durch deren „dramatische Diagnose“ ${ }^{“ 490}$ zum Akteur macht und als „tatkräftige[r] Krisenmanager “491 anbietet, sogenannte „,alternativlose“ Handlungszwänge dienen ihm [...] als Argument, das Heft in die Hand zu nehmen. "492 Auch Hempel und Markwart stellen fest, dass „Katastrophen-Sprechakte [...] als Souveränitätsbehauptungen konzipiert" sind. ${ }^{493}$ Schmidt rekurrierte in seinen Deutungen der Ereignisse dabei auf vergangene, im Gedächtnis der Stadt fest verankerte, Geschehnisse, konkret auf den Hamburger Brand von 1842 und auf den Hamburger Feuersturm im Jahr 1943. ${ }^{494}$ Der Appell zur Besinnung auf den Wiederaufbau, ermöglicht „durch hanseatische Zähigkeit“" 495 , entsprach einem Deutungsangebot, das nicht auf eine „negative Geschichte“ ${ }^{\text {496 }}$, sondern auf vorhandene Ressourcen fokussierte. Die damalige Berichterstattung nahm diese Sichtweise auf, die es zudem erlaubte, „die Komplexität der Lage“ auf eine Akteursgestalt „zu reduzieren“, bzw. war es auch Schmidt selbst, der, wie sein ehemaliger Mitarbeiter und Biograf Hartmut Soell herausstellt, „Journalisten relativ frühzeitig am Handeln und dessen Darstellung [...] teilnehmen ließ.“497 Die Massenmedien moderierten nicht nur den Prozess hin zu einer „(Wieder-)Herstellung geordneter Lebensverhältnisse“, sondern damit verbunden auch die Rückerlangung von „Normalität und die Bewältigung der Katastrophe ${ }^{\text {498 }}$.

\footnotetext{
488 Engels 2003, S. 128.

489 Ebd.

490 Meyer/Patzel-Mattern/Schenk 2013, S. 10.

491 Nünning, Ansgar: Grundzüge einer Narratologie der Krise. Wie aus einer Situation ein Plot und eine Krise (konstruiert) werden. In: Grunwald, Henning/Pfister, Manfred (Hg.): Krisis! Krisenszenarien, Diagnosen und Diskursstrategien. München 2007, S. 48-71, S. 64.

492 Meyer/Patzel-Mattern/Schenk 2013, S. 11.

493 Vgl. Hempel/Markwart 2013, S. 12.

494 Vgl. Schott 2002.

495 Helmut Schmidt an Erich Lüth (Staatliche Pressestelle), 15.03.1962. In: StAHH, 135-1 VI, 901, zit. nach Mauch 2015, S. 88 (StAHH = Staatsarchiv Hamburg).

496 Siehe hierzu Koselleck, Reinhardt: Formen und Traditionen des negativen Gedächtnisses. In: Knigge, Volkhard/Frei, Norbert (Hg.): Verbrechen erinnern. Die Auseinandersetzung mit Holocaust und Völkermord. München 2002, S. 21-32.

497 Soell 2003, S. 382.

498 Scholz, Tobias: Distanziertes Mitleid. Mediale Bilder, Emotionen und Solidarität angesichts von Katastrophen. Frankfurt a. M./New York 2012, S. 13.
} 
Die Hamburger Sturmflut von 1962 kann insgesamt als ein herausragendes mediales Ereignis angesehen werden, denn neben Zeitungen und dem Hörfunk, ist es 1962 erstmalig das Fernsehen, das die Geschehnisse bildreich in die bundesdeutschen Haushalte transportiert. Und Helmut Schmidt wird zur Hauptfigur dieser medialen Berichterstattung. Durch seine „diffuse Popularität weit über Hamburgs Grenzen hinaus“, wie Engels anmerkt, „erstritt er sich“ - durchaus telegen, wie auch das Archivmaterial im Film Flut 1962 - Erinnern. Gedenken. Erzählen. zeigt - „im öffentlichen Raum eine präzise Rollenzuschreibung, die den Macher-Kanzler der 1970er Jahre präfigurierte. “499

Für die Interviewpartner_innen stehen im Gegensatz dazu Personen im Mittelpunkt, die, aus dem lokalen Umfeld stammend, Hilfe geleistet haben, wie der damalige Wilhelmsburger Ortsamtsleiter Hermann Westphal, nach dem heute im Stadtteil eine Straße benannt ist. Weitere Personengruppen, die in den Interviews benannt werden, sind Mitarbeiter_innen des Roten Kreuzes und „Ärzte, die bis zur Erschöpfung, äh, in der Nacht [...] herumgefahren wurde[n] und hier Spritzen gab[en] und dort Medikamente gab[en]" und sich dadurch „sehr verdient gemacht" haben, wie Ernst Meyer die Situation in Wilhelmsburg beschreibt. Auch der Neuenfelder Polizeimeister Fritz Hilke findet ausdrückliche Erwähnung, da er dem Bruder einer Zeitzeugin das Leben rettete. Helmut Schmidt hingegen ist nicht Bestandteil der persönlichen Masternarrationen, er wird, nicht aus eigenem Impuls heraus, sondern erst auf meiner Nachfrage hin thematisiert. Zwei Aussagen sind hierfür beispielhaft. ${ }^{500}$

Elisabeth Schwartau aus Altenwerder etwa setzt ihre eigenen Schwerpunkte:

„Also am 17. und 18. ist es immer noch reingeströmt, und dann allmählich, ähm, haben die, Leute, die 'n LKW hatten und auch mit den Treckern haben sie dann erstmal Sand geholt und nachher kamen dann auch LKWs gefahren. Helmut Schmidt ist dann hier gewesen, hat dafür gesorgt, dass die Bundeswehr mithilft und dann haben sie Sandsäcke gefüllt und haben erstmal versucht die, ähm, Löcher wieder dicht zu machen. Ich weiß, dass ein Nachbar erzählte, er hat im Wohnzimmer gestanden, äh, und hat raus geguckt und dann hat er plötzlich gesehen, dass das Wasser nicht mehr, äh, höher wird, sondern dass es äh, wegläuft, und dann hat er gesagt, ,So, jetzt haben wir das Schlimmste hinter uns'. Und dann ist er in die Küche gegangen und wollte 'n Schnaps trinken und dabei hat er festgestellt, dass zwischen dem, ihm und dem Nachbarn der Deich gebrochen war. Und da war's denn ganz vorbei, da hat er seinen Schnaps nicht mehr getrunken. (lacht)“

Ingrid Kannen aus Wilhelmsburg, Ortsteil Kirchdorf, formuliert stellvertretend für viele der Befragten:

„Also gut, was man nachher im Nachherein gehört hat, aber damals so, zu der Zeit war wirklich nur so die nähere Umgebung, und das waren eigent-

499 Engels 2003, S. 128.

500 Beide Aussagen sind im Film nicht enthalten. Zu den Auswahlkriterien siehe Kapitel 3.2.3. 
lich eben die Leute, die geholfen haben, und äh, die man kannte, und die vor Ort waren. Aber alles andere war eigentlich sehr weit weg. So, Gedanken gemacht, wer das jetzt eigentlich alles so schnell organisiert hat oder so, hab ich leider nicht gemacht zu der Zeit. Ich hab's einfach so hingenommen und für toll empfunden, dass es so war."

Die persönlichen Masternarrationen sind auf die unmittelbaren, eigenen Erlebnisse fokussiert. Helmut Schmidt agierte für die Bevölkerung fast unsichtbar ,hinter den Kulissen'. Er veranlasste am Morgen des 17. Februar 1962 umfangreiche Hilfsmaßnahmen, insbesondere durch militärische Verbündete der Bundesrepublik, zu denen er aus seiner Zeit im Verteidigungsausschuss des Deutschen Bundestages Kontakt hatte. Durch eine umfängliche Berichterstattung erlangte er mediale Präsenz. Drei Wochen nach den Ereignissen wurde ein Gesetzentwurf für die Schaffung einer Behörde für Inneres der Hamburger Bürgerschaft vom Senat vorgelegt, Schmidt wurde im selben Jahr Innensenator. ${ }^{501}$ Somit war auch er in verantwortlicher Position, als in Hamburg sukzessive wieder Ordnung hergestellt wurde.

\subsubsection{Zusammenfassung: Erzählen als Wiederherstellung von Ordnung}

Bereits im direkten Anschluss an die Erlebnisse haben die Menschen damit begonnen, sich über das Geschehene auszutauschen. Diese Geschichten waren ein unbewusster Bestandteil der Aufräumarbeiten und Ausdruck der eigenen mentalen Verfassung. ${ }^{502}$ Inwieweit die Menschen traumatisiert waren, das ist im Nachhinein nicht zu bestimmen. ${ }^{503}$ Entscheidend für diese Untersuchung sind jedoch die Erfahrungen von existenzieller Not. Die Erschütterungen des seelischen Gleichgewichts kommen dabei einer inneren Unordnung gleich, hervorgerufen durch das Erleben einer Diskrepanz „zwischen bedrohlichen Situationsfaktoren und individuellen Bewältigungsmöglichkeiten " 504 . Als hilfreiche Zugänge für die Wiederherstellung einer inneren Ordnung, also einer Verarbeitung der Erfahrungen, gelten mentale Strategien mit dem Ziel, die Ereignisse zu verstehen, ihnen einen Sinn einzuschreiben bzw. sie als in Zukunft kontrollierbar zu interpretieren. ${ }^{505}$

Diese etwaigen Kompetenzen sind abhängig von den sozio-kulturell zur Verfügung stehenden Ressourcen, wie anhand von Konzepten zu ,Vulnerabilität und Resilienz' oben ausgeführt wurde. ${ }^{506}$ Eine dieser Ressourcen ist die Möglichkeit und Fähigkeit, sich anderen mitteilen zu können, etwa um tröstende Reaktionen oder

\footnotetext{
501 Vgl. Soell 2003, S. 392.

502 Vgl. Bendix 1990, S. 333.

503 Schätzungen von Expert_innen gehen davon aus, dass etwa 30 \% der Menschen nach den Erlebnissen einer sogenannten ,Naturkatastrophe‘ psychologische Betreuung benötigen. Vgl. Rieken 2010, S. 189; vgl. Sticher 2013, S. 327.

504 Topaloglou/Tschugguel 2015, S. 39f., mit Bezug auf Fischer, Gottfried/Riedesser, Peter: Lehrbuch der Psychotraumatologie. 3. Auflage. München/Basel, 2003, S. 82.

505 Vgl. Perren-Klingler 2004, S. 219.

506 Siehe ausführlich Kapitel 2.4; vgl. Perren-Klingler 2004, S. 222.
} 
Anerkennung für das Erlebte zu erhalten. Die Sprache fungiert dabei als Schnittstelle zwischen dem Individuum und den Mitmenschen. Dieses Mitteilen ist dann ordnend „ein Schritt in Richtung Selbstkohärenz."507 Wie die bereits erwähnte Untersuchung von Bendix aus dem Jahr 1990 herausgestellt hat, kann durch eine Strukturierung der Erlebnisse im kommunikativen Austausch demgemäß erzählend Ordnung wiederhergestellt werden. ${ }^{508}$ Mehr noch transformieren die Geschichten die Betroffenen „from being the helpless victim one becomes the effective story teller “. ${ }^{509}$ Das Erzählen hat demgemäß einen emanzipatorischen Effekt.

Die oben dargelegten Leitlinien des Erzählens können somit, wie Lehmann darlegt, als „Sinnsystem“ begriffen werden „das für den Sprecher und für seine Zuhörer Ordnung und Verständlichkeit in die disparaten Erfahrungen eines Lebens bringen soll." 510

Diese auf Ordnung ausgerichtete Erzählweise verdeutlicht, dass der strukturelle Charakter von Leitlinien „für die narrative lebensgeschichtliche Selbstvergewisserung konstitutiv ist“, wie Schröder anmerkt. ${ }^{511}$ Lucius-Hoene und Deppermann heben die Relevanz hervor, die eine heutzutage memorierte „faktische und emotionale Beteiligung an den Ereignissen“, in Kombination mit Wissensständen und Bewertungen hat. ${ }^{512}$ Für die Befragten hat sich im Laufe der Jahre eine bestimmte Perspektive auf das Geschehen verfestigt. Es ist eine Selbstpositionierung mit ,eigensinniger' Anmutung und kann als „Markenzeichen“ der Befragten angesehen werden. ${ }^{513}$ Dieses konstituierte sich bereits 1962, und mit Topaloglou und Tschugguel formuliert, als

„Solidarität und [eine] Solidargemeinschaft [...] dort, wo sich Menschen durch ein zufälliges Schicksal, wie eine Naturkatastrophe, in ,einer' Situation wiederfinden und durch die Gemeinsamkeit emotionale Bindungen zueinander entwickeln. Diese entfalten sich umso stärker, je bewegender und intensiver das geteilte Schicksal ist und dafür kein Fremd- oder Selbstverschulden vorliegt." 514

Das Bewusstsein mangelnder Schuld ist auch für die damaligen Ereignisse festzustellen. Laut einer ifas-Studie aus dem Jahr 1964 waren 51\% der Hamburger Bevölkerung der Meinung, dass die Katastrophe nicht zu vermeiden war und niemanden

\footnotetext{
507 Perren-Klingler 2004, S. 223.

508 „For those who experienced the quake, however, rendering and listening to entire stories was a crucial first step in regaining order after chaos." Bendix 1990, S. 345.

509 Wolfenstein 1957, S. 139, zit. nach Bendix 1990, S. 334.

510 Lehmann 1983, S. 18.

511 Vgl. Schröder 2005, S. 17.

512 Vgl. Lucius-Hoene/Deppermann 2004, S. 23.

513 Vgl. Topaloglou/Tschugguel 2015, S. 60.

514 Ebd., S. 59, mit Bezug auf Höffe, Otfried: Gerechtigkeit. Eine Philosophische Einführung. 3. Auflage. München 2007, S. 91.
} 
eine Schuld trifft. Dieses Ergebnis interpretieren die Demoskop_innen insbesondere als Ausdruck eines fehlenden Interesses an der Schuldfrage. ${ }^{515}$

Bereits wenige Tage nach der Sturmflut hatten neben Helmut Schmidt auch andere politische Akteure in Rückbezug auf den Hamburger Brand und den Hamburger Feuersturm - letzterer war 1962 nicht mit Schuldfragen assoziiert - den Wertekanon einer Wir-Gemeinschaft beschworen. Diese Implementierung einer Schicksalsgemeinschaft war zunächst darauf ausgelegt, ein Bewusstsein lokaler und nationaler Solidarität zu erzeugen. ${ }^{516}$ Bereits im direkten Anschluss an die Ereignisse wurden positive Aspekte, wie nachbarschaftliche Hilfsbereitschaft und das Engagement der Helfer_innen sowie Schmidt als erfolgreicher Krisenmanager medial etabliert, wie aus Presseberichten und Ansprachen hervorgeht. ${ }^{517}$ Darüber hinaus steht die in der Gesellschaft verankerten Meistererzählung über dieses Extremereignis im Kontext des damaligen "Narrativ[s] eines sozioökonomischen Aufschwungs“ der Nachkriegszeit. ${ }^{518}$ Auch aus heutiger Erzählperspektive werden von den Interviewpartner_innen kohärente und somit letztendlich erfolgreiche Geschichten konstruiert, dadurch dass die damaligen Extremereignisse vor dem Hintergrund eigener, heutzutage verbesserter Lebensbedingungen eingeordnet werden.

Die persönlichen Masternarrationen konstituieren sich demgemäß aus mehreren Faktoren. Heutige Grundlage ist das implizite Wissen der Befragten über die Strukturierung und die Inhalte von Erzählmustern, die sich sowohl bewährt haben, als auch eine gewisse Vorhersehbarkeit in sich tragen, also einem bestimmten Emplotment folgen. ${ }^{519}$ Hierbei schöpfen die Befragten aus einem gemeinsamen „Geschichtenrepertoire“. Meyer spricht in diesem Zusammenhang von „,[n]arrativ[er] Kompetenz“.520 Der Detailreichtum, die Stringenz und die unverschleierte Subjektivität einer Binnenperspektive markieren zudem die "Glaubwürdigkeit des Erzählten" ${ }^{221}$. Die Zeitzeug_innen behaupten sich durch die Artikulation ähnlicher Erzählmus-

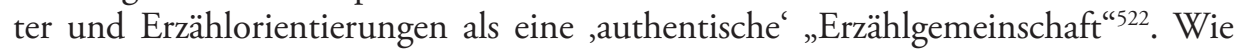
Löffler herausstellt, beanspruchen bereits „die knappen Erklärungs- und Argumen-

515 Vgl. Institut für angewandte Sozialwissenschaft: Die Flut. Ergebnisse einer Repräsentativerhebung in Hamburg. Winter 1962/63. Bad Godesberg 1964. Laut Demoskop_innen fehle es auch an Kenntnissen darüber, welche Institutionen überhaupt verantwortlich wären. Deichbauamt, Warndienste und Wetterdienst werden daher nicht benannt. Grundsätzlich sind in der Umfrage keine nennenswerten Unterschiede zwischen damals Betroffenen und der übrigen Hamburger Bevölkerung festzustellen.

516 Vgl. Schott 2002.

517 Vgl. Mauch 2014, S. 139.

518 Vgl. ebd.

519, ,I]t is the feel for what kind of narrative move leads to what next kind of move [...] I describe this tacit knowledge as predictable plot completions [...]. "Frank, Arthur W.: Letting Stories Breathe. A socio-narratology. Chicago/London 2010, S. 54. Hervorhebung im Original. „Menschen sind, das ist dabei wichtig hervorzuheben, nicht narrativ determiniert, sondern enkulturiert und sozialisiert." Meyer 2014, S. 251.

521 Ebd., S. 260.

522 Nünning 2013, S. 44. 
tationssätze der Topoi [...] Allgemeingültigkeit und Autorität.“ In ausgestalteten Geschichten gelte dies umso mehr, insbesondere wenn in ihnen Theorien und Wissensbestände gesammelt sind. ${ }^{523}$ Dieses Expert_innentum beinhaltet zudem explizite Abweichungen bzw. Auslassungen wesentlicher Bestandteile der in der Gesellschaft verankerten Meistererzählung, wie eine Betonung des Katastrophischen und der Rolle von Helmut Schmidt. Diese Gemeinsamkeiten der persönlichen Masternarrationen können somit insgesamt als ein nach wie vor von den Befragten miteinander geteilter Wertekanon interpretiert werden, der, trotz biografischer Unterschiede, für die Haltung gegenüber der Sturmflut von 1962 Gültigkeit hat.

Im dritten Teil des Films Flut 1962 - Erinnern. Gedenken. Erzählen. werden diese Haltungen als emanzipatorische Reflexionsstrategien im Kontext von Resilienz und Vulnerabilität plausibilisiert.

\subsection{Filmteil 3: Erzählen im Kontext von Resilienz und Vulnerabilität}

Im letzten Teil von Flut 1962 - Erinnern. Gedenken. Erzählen. stehen das Zusammenwirken von Erzählpraktiken mit einem distinktiven ,Lernen aus der Katastrophe‘ im Mittelpunkt. Das Erzählen, interpretiert als eine Strategie der Erfahrungsbewältigung, ist einer Resilienzstärkung dienlich, da es darauf ausgerichtet ist, „die eigene Geschichte auch auf Zukunft hin in einer kohärenten Weise" darzustellen, was Sedmak hervorhebt. ${ }^{524}$ In diesem Zusammenhang greift erneut die Argumentation von Pfister, der betont „memory is crucial for the development of coping strategies." 525

Bereits in den persönlichen Masternarrationen - thematisiert im zweiten Teil des Films - finden sich zentrale Sinnsysteme, die darauf schließen lassen, dass die Befragten ihre Erfahrungen im Kontext eigener Resilienz interpretieren. Ihre Erzählungen orientieren sich an Schilderungen sozialer Kompetenzen innerhalb familiärer und nachbarschaftlicher Netzwerke sowie von Disziplin und Selbstkontrolle. Exemplarisch hierfür beschreibt Adolf Kröger aus Finkenwerder seine Beteiligung an der lokalen Versorgung. Hieran ist erkenntnisleitend, dass er diese Aussage mit seiner damaligen Hilfsbedürftigkeit verknüpft:

„So, und dann war die erste Überlegung, wie viel Menschen sind wir in diesem Abschnitt, die versorgt werden müssen, weil also kein anderer Zugang da war, und dann hat man also das aufgenommen, und hat uns im Grunde genommen per Hubschrauber versorgt, mit warmem Essen. Es kamen Essenkübel, die also ein Etikett dran hatten, Dortmund. Es kam Butter, es kam

\footnotetext{
523 Vgl. Löffler 1999, S. 98.

524 Vgl. Sedmak 2013, S. 27.

525 Pfister 2011, S. 3.
} 
Wasser, das war das Allererste, was wir haben wollten, wir hatten ja nichts zu trinken, wir konnten ja also das Elbwasser, das war unmöglich zu trinken, und Reserven hatten wir auch nicht, und wenn wir 'n bisschen also in Flaschen hätten, das stand im Keller, da konnten wir ja nicht ran, das war also so. Also von der Seite her sind wir eigentlich sehr, sehr (betont) gut versorgt worden."

Diese „Wir“-Schilderung benennt soziale Beziehungen, innerhalb derer „Organisationsmuster, Kommunikationskultur, Problemlösungsstrategien und positive Orientierungssysteme" zutage treten. ${ }^{526}$ Die Interviewpartner_innen scheint zudem ein Wertekanon zu einen, wie bereits angedeutet wurde. Im dritten Filmteil wird diese Erzählperspektive durch szenische Filmaufnahmen mit den Befragten in ihrem heutigen Lebensumfeld zusätzlich betont. Die für diesen Teil ausgewählten Interviewausschnitte stammen jeweils aus der Nachfrage- und der Bilanzierungsphase der Interviewerhebung. ${ }^{527}$

\subsubsection{Erzählhaltungen zwischen Erfahrungswissen und Risikobewusstsein}

Der Übergang vom zweiten zum dritten Filmteil ist gekennzeichnet durch zeitgenössische Filmaufnahmen, die beispielhaft Wiederaufbaumaßnahmen in Neuenfelde zeigen, interpretiert als Sinnbild für die Wiederherstellung von Ordnung. ${ }^{528}$ Den Beginn dieses letzten Filmteils bildet eine Aufblende zu Adolf Fick, Obstbauer aus Finkenwerder, der bei ordnenden Tätigkeiten auf seinem Hof gezeigt wird: dem Sortieren seiner Apfelernte. Diese heutigen Filmbilder veranschaulichen Entwicklungen zu einem modernen Obstanbaubetrieb und setzen sie in Beziehung zu seinen Erinnerungserzählungen über den damaligen Wiederaufbau, die von Frustration, Mühen und Zweifeln geprägt sind.

Im Film sind die Äußerungen von Adolf Fick wie folgt zusammengefasst:

„Ich weiß nicht ob das der Anlass war [...] aber ich weiß nicht, ob das jetzt das Erlebnis von '62 war [...] Und, äh, wir haben dann auch, äh, unsere Betriebsform auch umgestellt. [...] Sonst hatten wir ja immer Landwirtschaft und Obstbau noch, und dann haben wir uns ganz auf Obstbau, äh, spezialisiert. [...] Und somit ist es schon, also wenn ich das aus meiner heutigen Sicht sehe, äh, dann ist das natürlich ganz schön deprimierend, weil, äh, wenn man so um die Mitte 50 ist, und da wird alles auf mal zerstört, dann ist es schon deprimierend, nich. Denn sagt man, ,Mensch, was machen wir nun?`. [...] Das ist ja so, es ist ja nachher ne Entschädigung gelaufen, und [...] Aber wie gesagt es ist viel ersetzt worden. Äh, wir haben günstige Kredite bekommen, aber es ist eben so, äh, letzten Endes steht man selber davor, und das ist natürlich so, dass man diese, ja, alles, ähm, das schmeißt einen wohl

526 Vgl. Sedmak 2013, S. 31f.

527 Ausführlich zur Interviewerhebung siehe Kapitel 3.1.2.

528 Ausschnitte aus dem Film ,Die große Flut' (Baubehörde - Tiefbauamt (LBH: Sign. 4200128)). 
20 Jahre wieder zurück. Das, was man neu wieder investieren muss, und sich wieder neu verschulden muss. [...] Und, äh, man kann's nicht beschreiben, das ist eben so, mit der Hoffnung, dass es weiter geht."

Die Bild- und Tonebenen wirken an dieser Stelle nicht nur komplementär, die filmszenisch ausgestalteten Tätigkeiten liefern darüber hinaus konkrete Hinweise darauf, dass „es weiter“ gegangen ist bzw. legt die Filmmontage heutige - der geäußerten finanziellen Neuverschuldung zum Trotz - verbesserte ökonomische Lebensverhältnisse nahe.

Neben einer monetären Unterstützung durch die Stadt Hamburg und den Bund werden für die Bewältigung der damaligen Geschehnisse von den Befragten eigene innere Haltungen sowie ein sozialer Zusammenhalt als wesentlich reflektiert. Den Umgang mit den Erfahrungen von Zerstörung und Verlust vor dem Hintergrund einer wiedererlangten Selbstkontrolle, also einer Abkehr von der Opferrolle, fasst im Film stellvertretend für die Haltung damals junger Erwachsener Gerhard Felka aus Wilhelmsburg, Ortsteil Georgswerder, zusammen. Diese Aussage ist ebenfalls filmszenisch im Kontext bereits hergestellter Ordnung inszeniert, hier am Beispiel seiner gärtnerischen Tätigkeiten:

„Das war- und äh, wie=wie, wie sie heute sagen, ,Steh auf, wenn Du'n Schalker bist', ne, und so war das auch. Äh, wir waren am Boden zerschlagen, wir waren aber nicht nervlich, wir waren aber nicht so kaputt, dass wir gesagt haben, ,das hat ja doch keinen Zweck, was soll das?", so waren wir nicht. [...] Wir waren Kämpfernaturen, wir haben das alles verloren und haben gesagt, ,So, jetzt müssen wir wieder neu anfangen.' [...] Sie müssen normal weiterleben können, sonst gehen Sie kaputt. [...] Sonst würden Sie sich, glaube ich, äh, selbst, äh, nervlich zerfleischen, wenn Sie das immer im Hinterkopf haben würden, das würde dann, das würde kein Leben sein, wenn Sie das nicht abschalten können, das würde kein Leben sein."

Neben Adolf Fick vergleicht auch Marlies Wenzel, wohnhaft in Wilhelmsburg, Ortsteil Kirchdorf, ihre Erfahrungen des Wiederaufbaus mit eigenen Erfahrungen nach dem Zweiten Weltkrieg. ${ }^{529}$ Im dritten Filmteil wird mit ihr ein weiteres Mal szenisch eine Interviewaussage betont, hier mit dem Ziel, das Gesagte zu unterstützen und zu veranschaulichen. Das Publikum erhält in dieser Szene eine Vorstellung davon, was Frau Wenzel vor ihrem inneren Auge sieht, nämlich ihren Garten, in dem sie es sich zusammen mit ihrem Ehemann Herbert Wenzel „schön“ gemacht hat:

„Es ist einfach schön hier, wir haben uns das ja aufgebaut nach dem Krieg, als ich hierher kam, als wir geheiratet haben, da war alles noch so kaputt vom

529 Lehmann definiert die Gattung des Vergleichs als „meistens zweigliedriges, meistens kurzes, thesenhaft abstrahierendes, in Oppositionen angelegtes Mittel der Erkenntnis, der Klärung von moralischen oder rechtlichen Positionen, der Veranschaulichung und Unterhaltung in Gesprächssituationen“. Lehmann 1991, S. 198. 
Krieg und so, und wir haben da so viel, so viel Arbeit rein gesteckt, so viel Geld rein gesteckt und alles, da hat man gar nicht dran gedacht hier weg zu gehen oder irgendwie. Das ist eben nur diese Angst eben immer noch, wenn die Sturmflut droht. Die werd' ich auch nicht mehr los. Auch wenn ich in der Stadt wohnen würde, würde ich die nicht loswerden. [...] Also jetzt bin ich soweit, ich weiß, wenn wir Süd-West-Sturm haben, schadet das nichts. Wenn ich dann im Radio höre, der Wind soll noch rum gehen auf Nord-West, also ich schlaf dann die ganze Nacht nicht. Ich bleib die ganze Nacht dann wach."

Marlies Wenzel verknüpft mit ihrer Argumentation zugunsten eines Wiederaufbaus heutige Vorbehalte in punkto Sicherheit. In diesem Zusammendenken wird ein Risikobewusstsein deutlich, das typisch ist für die hier befragten Zeitzeug_innen.

\subsubsection{Angst als Argument}

Risikobewusstsein interpretiere ich in Anlehnung an Felgentreff und Glade als das Ergebnis eines „Lerneffekt[es], basierend auf den noch abrufbaren Erinnerungen“, also auf einem Erfahrungswissen, das auch „Handlungsroutinen“ im Fall einer erneuten Überflutung zur Verfügung stellen kann. ${ }^{530}$

Besonders wichtig ist in diesem Zusammenhang die Umdeutung von Erfahrungen, die vermutlich bereits mit den ersten, die Erlebnisse ordnenden Erzählungen eingesetzt hatte. Helga Rostowski, die als Kind im Wilhelmsburger Ortsteil Georgswerder in einer Behelfsheimsiedlung massiv von den Wassermassen bedroht war, steht exemplarisch für diesen Umdeutungs- respektive Bewältigungsprozess. Ihre Reflexionen sind im Film wie folgt zusammengefasst:

„Und ich für meinen Teil denke immer, [...] wenn das Wasser, das würde, da würden wir uns auf jeden Fall vor retten können. [...] So hoch kann das gar nicht kommen, ich wüsste ja jetzt viel eher, was ich machen würde und äh, wäre viel hellhöriger auf alles. Also insofern denke ich, ja, [...] ich find's nach wie vor eigentlich schön hier zu wohnen, grad eben Kirchdorf."

Aus den Erfahrungen als Opfer erwuchs durch eine Uminterpretation „kulturell präfigurierte[r] ,Opferrollen ““331, eine Geschichte der aktiven Gestaltung und somit eine durch Erzählungen erfolgte Aneignung dieser Extremsituation. Zu diesem konstruktiven Umgang gehört auch das unumwundene Eingeständnis von heutiger Ängstlichkeit, wie dies auch Marlies Wenzel thematisiert. Die als hierfür exemplarisch interpretierte Haltung von Ursula Seemann aus Wilhelmsburg, Ortsteil Reiherstieg, ist im Film folgendermaßen zusammengefasst:

530 Vgl. Felgentreff/Glade 2008, S. 444.

531 Simon, Michael: Ethnologische Anmerkungen zu Bernd Riekens „Gesprächen mit Einheimischen“. In: Rieken, Bernd (Hg.): Wie bewältigt man das Unfassbare? Interdisziplinäre Zugänge am Beispiel der Lawinenkatastrophe von Galtür. Münster 2015, S. 93-105, S, 96. 
„Und das ist ja die Angst, mit der wir hier leben. [...] Wann kommt das nächste, wann kommt die nächste Flut, und bei jedem, äh, ja bei jedem stärkeren Wind, was auch in den Orkan rein ging, hatte man ja Angst. [...] Und ich glaube so diese alten Wilhelmsburger, die das, äh, miterlebt haben, wenn Sie da mal so richtig hinhorchen, die haben, glaube ich, alle Angst noch."

Die bewusste Entscheidung vieler Zeitzeug_innen ihrer „Angst“ zum Trotz an dem Ort des damaligen Geschehens wohnen zu bleiben, gründet sich auf Freiwilligkeit, also vermutlich nicht primär auf einer pragmatischen bis resignativen und somit „realistische[n] Sicht, die Common Sense ist auch in anderen, vergleichbaren Siedlungslagen "532, die mit einem Hochwasserrisiko belastet sind, sondern vielmehr auf der erfahrungsbasierten Abwägung von Risiken zugunsten eines Gefühls der Beheimatung. ${ }^{533}$ Auch Ursula Seemann rekurriert somit letztendlich in ihren Äußerungen auf wesentliche Faktoren einer Resilienzstärkung, wenn sie auf die sozialen Beziehungen der lokalen Bevölkerung („diese alten Wilhelmsburger“) hinweist.

Auf eine emotional grundierte Ortsbezogenheit beziehen sich sowohl Helga Rostowski („Ich find's nach wie vor eigentlich schön hier zu wohnen, grad eben Kirchdorf [... .“) als auch Marlies Wenzel („Es ist einfach schön hier.“). Und Adolf Kröger aus Finkenwerder fasst diese Haltung gewissermaßen zusammen, es ist die letzte Aussage im Film: „Unsere Familien, meine noch länger als die Familie meiner Frau, ist hier in Finkenwerder seit 1624 beheimatet, da zieht man nicht weg. Da bleibt man."

Aus diesen Äußerungen spricht eine insgesamt wertebewahrende Haltung. Hintergrund dafür ist ebenfalls ein ausgeprägtes „Landschaftsbewusstsein“, also eine Identifikation mit bestimmten Orten, die bis in die heutige Zeit nachhaltig ist. ${ }^{53}$ Zudem zeigt sich der Wunsch nach Verhaltenssicherheit, also „sich solche Plätze und soziale Milieus mental zu sichern, mit denen sie sich lebensgeschichtlich identifizieren können", ermöglicht durch die Beständigkeit des Bekannten und Gewohnten. ${ }^{535}$ Dies gilt umso mehr wenn dort Extremereignisse durchlebt wurden.

Die Ortsbezogenheit in den Erzählungen der Befragten kann somit als ein für die Resilienz relevanter Faktor interpretiert werden, nämlich „im Spannungsfeld von Beharrung und Veränderung“, hier mit eben jenem Schwerpunkt auf dem „Beharrungsvermögen“ der Menschen. ${ }^{536}$ War also die erste Zeit nach den Extremereignissen im Februar 1962 zunächst geprägt von der Notwendigkeit, mit unmittelbaren Veränderungen umzugehen und diese zu meistern, so sind es insbesondere die anschließende, jahrzehntelange lokale Verbundenheit und eine soziale Beständigkeit, die eine die Resilienz stärkende Wirkung begünstigen.

\footnotetext{
532 Rieken 2010, S. 194.

533 Siehe hierzu auch Sticher 2013, S. 311.

534 Vgl. Lehmann 2003, S. 159: „Das Landschaftsbewußtsein gehört zu den konservativen Elementen unserer Kultur."

535 Vgl. ebd., S. 149, mit Bezug auf Greverus, Ina-Maria: Der territoriale Mensch. Ein literaturanthropologischer Versuch zum Heimatphänomen. Frankfurt a. M. 1972.

536 Vgl. Bonß 2015, S. $17 f$.
} 


\subsubsection{Lokalität und Resilienz}

Die Aussagen der Protagonist_innen im dritten Filmteil über damalige Vorbehalte in Bezug auf einen gelingenden Wiederaufbau, über mentale Strategien der Bewältigung, über Beheimatung und insgesamt über die Relevanz von Erinnerungen an die Geschehnisse werden durch die filmische Gestaltung von Flut 1962 - Erinnern. Gedenken. Erzählen. hervorgehoben. Herr Fick und Herr Felka ordnen gewissermaßen ihre wiederhergestellte Ordnung - der eine sortiert und transportiert seine erwirtschafteten Obstbestände, der andere jätet sein Gemüsebeet - und dies geschieht an den Orten damals erlebter Extremerfahrungen. Während Frau Wenzel sich gemeinsam mit ihrem Ehemann an ihrem mittlerweile schön hergerichteten Garten erfreut, verweilt Herr Kröger am Ufer der als Hochwasserschutzmaßnahme nach 1962 in Finkenwerder eingedeichten Süderelbe. Auch er hat sich mit dieser Veränderung der Landschaft als hazardscape arrangiert und sie auf seiner Route für einen Spaziergang integriert. ${ }^{537}$

Neben diesen privaten, persönlich konnotierten, Orten der Erinnerung können auch lokale Artefakte, die im Nachhinein an öffentlichen Orten errichtet wurden, hier etwa Denkmäler oder Hochwasserschutzanlagen, von Bedeutung für die individuellen Erinnerungen an die Sturmflut von 1962 sein, ebenso wie anschließende städtebauliche Veränderungen. ${ }^{538}$ In ihrem Zusammenwirken bilden sie Gedächtnislandschaften aus, die eine nachhaltig atmosphärische Wirkung auf die Erzählungen der Befragten entfalten. Dies gilt auch für seither auftretende Wetterlagen, insbesondere Sturm bzw. dadurch bedingte Sturmfluten. ${ }^{539}$

Auch ritualisierte erinnerungskulturelle Praktiken erfüllen diese identitätsstiftende Funktion. Gerhard Wendt aus Wilhelmsburg, Ortsteil Kirchdorf, betont: „Für uns ist es wichtig, dass wir also sagen, in Wilhelmsburg, in dem Gebiet, wo wir also betroffen waren, wir müssen einfach dran erinnern."

Als dramaturgische Klammer fungieren im Film öffentliche Gedenkorte. $\mathrm{Zu}$ Beginn ist es eine Gedenkveranstaltung am Deichdenkmal an der Kirchdorfer Straße in Wilhelmsburg. Am Filmende sind weitere, modernere Gedenkorte integriert, wie das ehemalige Flutdenkmal am Vogelhüttendeich in Wilhelmsburg - das vormals als Brunnenensemble am Stübenplatz errichtet worden war und einer Umgestaltung des Platzes weichen musste - sowie das 2002 errichtete Denkmal für die Flutopfer aus Francop und Neuenfelde. Diese „Denkmäler in der Zeit" ${ }^{\text {" }} 40$ schaffen Anlässe regelmäßiger gemeinsamer Partizipation, sie ermöglichen Wir-Inszenierungen, die

537 Mauch definiert hazardscapes als „Zusammenspiel von Naturgefahren, Verwundbarkeit und kultureller Adaption“. Mauch 2015, S. 27.

538 Ausführlich zu städtebaulichen Veränderungen in den damaligen Überflutungsgebieten siehe Kapitel 4.2.4.

539 „Die Sturmflut erzählt folglich auch von einer Region, in der Fluten bis heute unter bestimmten Umständen die Norm und ein strukturierendes Merkmal der Landschaft sind. " Mauch 2014, S. 135 .

540 Assmann, Aleida: Jahrestage - Denkmäler in der Zeit. In: Münch, Paul (Hg.): Jubiläum, Jubiläum ... Zur Geschichte öffentlicher und privater Erinnerung. Essen 2005, S. 305-314. 
gleichzeitig auch Anstöße zur persönlichen Reflexion, insbesondere durch „regelmäßige Wiederkehr und starke Ritualisierungen“ eröffnen. ${ }^{541}$ Entscheidend ist hierbei die Interdependenz der symbolisch an diesen Orten und zu diesen Anlässen praktizierten Haltungen der Anwesenden: einerseits das Wissen über ein anhaltendes Bedrohungspotenzial durch Sturmfluten und andererseits ein alljährlich zur Erinnerung gebrachtes Bewusstsein darüber, das vergangene Extremereignis bewältigt zu haben, also letztendlich einer Emanzipation von der Opferrolle. ${ }^{542}$

Diese im dritten Filmteil fokussierten Bedeutungsebenen von lokaler Verbundenheit und einer sozialen Beständigkeit, also einer Ortsbezogenheit, interpretiert als ein „Wunsch nach Heimat“543, eröffnet Erkenntnisse über die Vulnerabilität dieser Erinnerungsgemeinschaft. Bereits die Freiwilligkeit der Entscheidung, vor Ort, in einem prinzipiell überflutungsgefährdeten Gebiet, wohnen zu bleiben, also dem Wissen darüber, „mit Unsicherheit zu leben“544 kann als eine Haltung zur Resilienzstärkung angesehen werden. Hinzu kommt ein Bewusstsein von agency, also darüber, sowohl in einer Extremsituation als auch zu deren Bewältigung ein „Verhaltensrepertoire und Handlungsreservoir" ${ }^{455}$ zur Verfügung zu haben, wie die Beschreibung der damaligen Versorgungsabläufe von Adolf Kröger als auch die Einschätzungen von Helga Rostowski nahelegen. Dem zugrunde liegt eine innere Haltung über die Relevanz und über die Kohärenz des eigenen Tuns, eine Sinnhaftigkeit, die sowohl individuell als auch für diese Erzählgemeinschaft identitätsbildend wirken kann. ${ }^{546}$

Entscheidend ist jedoch, wie Sedmak zusammenfasst, „die Einsicht, dass resilient nicht die Person ist, die nicht berührt und verletzt werden kann, sondern die Person, die um die eigene Verwundbarkeit weiß und auch beschädigt werden kann. " ${ }^{447}$ Wenn die Befragten also mehrfach artikulieren, dass sie nach wie vor „Angst“ haben oder sie „unruhig“ werden, wenn eine erneute Sturmflut droht, wie auch Elisabeth Schwartau aus Altenwerder im Film eingesteht, so interpretiere ich dies eben nicht als Ausdruck von Vulnerabilität, sondern von Resilienz, nämlich als Beleg für ein angemessenes Risikobewusstsein in Bezug auf zukünftige Gefährdungslagen.

541 Vgl. ebd., S. 310f.

542 Vgl. Rheinheimer, Martin: Mythos Sturmflut. Der Kampf gegen das Meer und die Suche nach Identität. In: Demokratische Geschichte. Jahrbuch für Schleswig-Holstein 15, 2003, S. 9-58, S. 49.

543 Lehmann 2007, S. 149. Die Definition von Ortsbezogenheit führt Lehmann dahingehend aus, dass „primär die Sozialzusammenhänge der Bewohner bestimmter Orte bzw. Regionen [...] die Bindung an eine Wohngemeinde, d. h. ihre Ortsbezogenheit bewirken“. Lehmann 2007, S. 149, mit Bezug auf Treinen, Heiner: Symbolische Ortsbezogenheit. In: Kölner Zeitschrift für Soziologie und Sozialpsychologie 17 (1965), Heft 1, S. 73-97 sowie Heft 2, S. 254-297.

544 Sedmak 2013, S. 20.

545 Ebd.

546 Vgl. ebd. Im Kontext einer Gefühlen von Beheimatung inhärenten wertebewahrenden Haltung besteht allerdings auch die Gefahr einer ausgrenzenden Moralisierung, wie Sedmak ergänzt, da Gruppen sich auch in Abgrenzung zu anderen definieren. Vgl. ebd., S. 23.

547 Ebd., S. 34. 


\subsubsection{Cultures of Awareness}

Gefühle von Angst werden also durchaus reaktiviert, wenn witterungsbedingt Situationen auftreten, die an die damaligen Gegebenheiten erinnern. Den „Stellenwert der Angst" interpretiere ich allerdings nicht „,als dauerhafte Struktur " ${ }^{\text {“48 }}$, sondern als einen weiterführenden Bestandteil von, wie ich dies nenne, Cultures of Awareness. ${ }^{549}$ Volkskundlich-kulturanthropologische Perspektiven auf Wandel und subjektive Deutungspraktiken eröffnen Untersuchungen zu Erfahrungswissen und Risikobewusstsein und somit Anknüpfungen an Forschungsdiskurse zu Resilienz und Vulnerabilität, deren analytische Zugänge sowohl zur Untersuchung von individuellen als auch für kollektive Zuschreibungen Verwendung finden können. ${ }^{550}$

Die von Bonß prognostizierte und geforderte „neue [...] Kultur der Unsicherheit“, als ein, wie bereits in Kapitel 2.4 dargelegt wurde, „prinzipielles Bekenntnis zu Unsicherheit als Basis und Bezugspunkt menschlichen Lebens bei gleichzeitiger

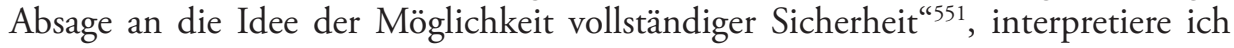
daher nicht nur als ein Plädoyer für einen zukünftigen Umgang mit Extremereignissen. Die Analyseergebnisse der erhobenen Erinnerungserzählungen haben vielmehr gezeigt, dass dies innerhalb einer gefährdeten Bevölkerung bereits gelebte Praxis ist. Die Interviewaussagen interpretiere ich entsprechend als Ausdruck von Resilienz, nämlich als Fähigkeit „zu antizipieren, zu reagieren, sich zu wehren und sich von der möglichen Realisierung eines unwägbaren Ereignisses zu erholen. "“552

Die Frage einer kulturwissenschaftliche Bewusstseinsanalyse danach, ob die „Menschen im Alltag ein Gefühl ihrer eigenen Geschichtlichkeit " ${ }^{\text {"553 }}$ besitzen, interpretiere ich als eine weitere Untersuchungsperspektive von Cultures of Awareness. Erinnerungserzählungen offenbaren eine historische Dimension, indem sie sich, um eine „kulturelle Anschlußfähigkeit“ zu gewährleisten, ,auf vorhandene Erzählstrukturen [...], kollektive Deutungsmuster, Metaphern, Kollektivsymbole u. a. m." ${ }^{554}$ zurückgreifen, wie sie in der Meistererzählung bereitgehalten werden. ${ }^{55} \mathrm{Mehr}$ noch vermitteln die Befragten den Eindruck eines Bewusstseins davon, dass ihre Erfahrungsgeschichten eine gesellschaftliche Relevanz haben. Dies wird weniger innerhalb der erhobenen

\footnotetext{
548 Rieken 2007a, S. 44.

549 Auch der von Sticher verwendete Terminus, Risikowahrnehmung' impliziert noch keine Handlungsorientierung, wendet sich aber ebenfalls gegen eine Viktimisierung, denn dieser gründe sich, wie Sticher ausführt, auf einen „aktiven Verarbeitungsprozess unter Rückgriff auf eigenen Erfahrungen, Wissen, Werte und Wünsche“. Sticher 2013, S. 309.

550 Vgl. hierzu auch Rieken 2015, S. 121.

551 Bonß 2010, S. 65.

552 Walter 2010, S. 16.

553 Vgl. Lehmann, Albrecht: Homo narrans - Individuelle und kollektive Dimensionen des Erzählens. In: Brednich, Rolf Wilhelm (Hg.): Erzählkultur. Beiträge zur kulturwissenschaftlichen Erzählforschung. Hans-Jörg Uther zum 65. Geburtstag. Berlin/New York 2009, S. 60-70, S. 67.

554 Keller 2000, S. 276.

555 Siehe hierzu ausführlich Kapitel 2.1 und Kapitel 4.2.
} 
persönlichen Masternarrationen deutlich, als vielmehr in den Nachfrage- und Bilanzierungsphasen der Interviews. Dieser Haltung wird im dritten Teil von Flut 1962 Erinnern. Gedenken. Erzählen. entsprochen. Mit ihren Aussagen zu einer potenziellen Bedrohung der Gebiete durch Hochwasser wird die filmisch erzeugte Erzählgemeinschaft in einer mahnenden und damit auf die Zukunft hin orientierten Position dargestellt.

Gerhard Wendt aus Wilhelmsburg, Ortsteil Kirchdorf, formuliert:

„Es sollte vielleicht noch 'n bisschen mehr bekannt gemacht werden, damit auch Leute, die also hier wohnen, sich das in Erinnerung rufen und sagen, „Mensch, das ist hier passiert.', das kann, also könnte, theoretisch kann das irgendwann wieder passieren, wie auch immer, auch wenn wir Schleusen hier hatten oder was noch höher kommt, was wir alle nicht hoffen wollen. Aber das Bewusstsein, dass ich in einem Gebiet lebe, wo also so was passiert ist, und eigentlich auch wiederkommen könnte."

Ein Geschichtsbewusstsein, das sich nicht nur auf die Vergangenheit bezieht, sondern, wie Rüsen anmerkt, „zugleich bestimmt ist durch Zukunftserwartungen und Orientierungsabsichten im Blick auf gegenwärtige Lebensverhältnisse “556, bietet somit womöglich sogar den Grundstein zur Untersuchung von Cultures of Awareness, nämlich in Artikulationen eines Risikobewusstsein, das sich sowohl aus dem Erfahrungswissen von Zeitzeug_innen speist als auch aus sozialen Verbundenheiten. Darüber hinaus eint die Befragten der Wunsch „to voice their concern“557, also eine Bereitschaft zu zivilgesellschaftlicher Partizipation.

Daran anknüpfend möchte ich drei Aspekte herausstellen, die mir in diesem Zusammenhang besonders bedenkenswert erscheinen:

Erstens kann gerade die souveräne Selbstrepräsentation der Zeitzeug_innen in Bezug auf ihr Erfahrungswissen eine wertvolle Ressource zur Vermittlung von Anpassungs- und Bewältigungsstrategien in den Diensten einer mit Naturrisiken konfrontierten Gesellschaft sein. Zeitzeug_innen tragen somit auch Verantwortung, sich als Teil der Gemeinschaft einzubringen und ihr Erfahrungswissen zur Verfügung zu stellen. ${ }^{558}$ Daher plädiere ich zweitens dafür, Zeitzeug_innen als zentralen Bestandteil von Cultures of Awareness zu integrieren, da sie innerhalb einer auf die Gegenwart und die Zukunft gerichteten Partizipation an der kulturellen Produktion und Verhandlung von Deutungen vermittelnd wirken können und ihnen eine Vorbildfunktion zukommt. ${ }^{559}$ Und drittens: Um den aktuellen Herausforderungen

556 Rüsen, Jörn: Einleitung. Geschichtsbewußtsein thematisieren - Problemlagen und Analysestrategien. In: Ders. (Hg.): Geschichtsbewusstsein: psychologische Grundlagen, Entwicklungskonzepte, empirische Befunde. Köln/Weimar/Wien 2001, S. 1-13, S. $10 f$.

557 Kapucu/Hawkins/Rivera 2013, S. 9.

558 Zur Kritik an sog. „Schadensausgleichmechanismen“, etwa durch staatliche Hilfen und Versicherungen, die eine Eigenverantwortlichkeit schmälern können, vgl. Felgentreff/Glade 2008, S. 444.

559 Fellmer erhebt anhand ihrer Untersuchungsergebnisse die Forderung, „dass seitens der staatlichen Küsten- und Katastrophenschutzverwaltungen mehr Möglichkeiten zur zivilgesell- 
im Umgang mit Naturrisiken und -gefahren zu begegnen, läge es somit nahe, auch das Erfahrungswissen der betroffenen Bevölkerung anderer ,Naturkatastrophen stärker qualitativ-interpretativ und transdisziplinär zu untersuchen. ${ }^{560}$ Auch wenn die hier erhobenen Erzählungen ein Extremereignis aus großem zeitlichen Abstand thematisieren, so liegt die Vermutung nah - und Bendix' Studie von 1990 bestätigt dies - dass sowohl in Bezug auf kürzer zurückliegende Extremereignisse als auch mit direkt im Anschluss erhobenen Interviews, hier relevante Ergebnisse einer Erzählforschung als Bewusstseinsanalyse erzielt werden können.

Das Erfahrungswissen und das Risikobewusstsein der im Film dargestellten Protagonist_innen konstituieren sich aus ihren lokalen Erlebnissen in jenen Tagen. Im Kontext der im Rahmen dieser Untersuchung dargestellten Konzepte von Vulnerabilität und insbesondere von Resilienz konnten weiterführende Erkenntnisse gewonnen werden. Mit ihren Äußerungen im dritten Filmteil verweisen die Befragten sowohl auf ihre gegenwärtige Haltung als auch auf das Wissen um potenzielle Risiken und Gefahren. Lokale Resilienz beinhaltet demgemäß auch die bodenständige Artikulation und Repräsentation eines ,Nichtsdestotrotz', bei dem es gerade nicht darum geht „die Fähigkeit, Hindernissen auszuweichen oder Widrigkeiten zu leugnen "561. Vielmehr sind die - hier filmisch interpretierten - Äußerungen Ausdruck davon, dass es den interviewten Zeitzeug_innen gelungen ist, ihr Erfahrungswissen in ein Risikobewusstsein zu transformieren, das, im wahrsten Sinne des Wortes, vielerlei Gesichter hat.

schaftlichen Mitgestaltung im Sturmflutschutz geschaffen werden sollten. " Fellmer 2014, S. 258.

Beispielhaft zu nennen ist eine ethnographische Untersuchung der Universität Rom „Sapienza“ zum Erdbeben in L'Aquila im Jahr 2009. Sie verfolgte das Ziel, „die individuelle und kulturelle Identität der Opfer des Erdbebens zu stärken“ und wurde von lokalen Vereinen unterstützt. Vgl. Simonicca, Alessandro: Das Erdbeben von L'Aquila, 6. April 2009. Zwischen Kultur der Katastrophe und politischem Handeln. In: Braun, Karl/Dieterich, Claus-Marco/Treiber, Angela (Hg.): Materialisierung von Kultur. Diskurse Dinge Praktiken. Würzburg 2015, S. 459-465, S. 462.

Sedmak 2013, S. 16. 


\section{Fazit: Erzählforschung und Film - Warum Worte Bilder brauchen}

Mit dem Zusatz in dieser Überschrift „Warum Worte Bilder brauchen“ nehme ich erneut Bezug auf die Forschungsberichte in den Dissertationen von Lisa Röösli und Marius Risi. ${ }^{562}$ Letzterer argumentiert in seiner Einleitung unter der Überschrift „Warum Bilder Worte brauchen"563 für kulturwissenschaftliche Filme ergänzende, schriftliche Ausführungen, um (s)einem Forschungsfilm, unter die Arme zu greifen'. ${ }^{564}$ Die Argumentation des Fazits von Risi umzudrehen, stellt indes keinen Widerspruch dar. Vielmehr habe ich mit meinen Darlegungen versucht, diesem Paradigma in großer Ausführlichkeit zu entsprechen. Nichtsdestotrotz überwiegt hier als grundsätzliches Anliegen, Film als ein Wissensformat zu nutzen, das im Sinne MacDougalls eine Entwicklung filmspezifischer Zugänge betont und von denen das Fach insgesamt profitieren kann. MacDougall spricht von der Notwendigkeit, „to build an intellectual foundation for visual anthropology by enabling a shift from word-and-sentence-based anthropological thought to image-and-sequence-based anthropological thought", auch vor dem Hintergrund,

\footnotetext{
$562 \mathrm{Zu}$ den Untersuchungen von Risi und Röösli siehe Kapitel 1.

563 Risi 2010, S. 149.

564 Vgl. ebd., S. 152.
} 
dass filmisches Arbeiten nicht als ein Ersatz für verschriftlichte Untersuchungen angesehen werden sollte. ${ }^{565}$

Obwohl der Film Flut 1962 - Erinnern. Gedenken. Erzählen. mittlerweile nicht mehr gängigen Standards der Film- und Fernsehtechnik entspricht - aufgrund seines 4:3-Formats und weil er nicht in HD produziert wurde - hat er ein großes Publikum erreicht. Sein „evokatives“ Potenzial entfaltet auch dieser Film bislang innerhalb verschiedener Veröffentlichungsarenen. ${ }^{566}$ Er wurde wiederholt gezeigt, etwa dreimalig im Februar und März 2008 in einer sonntäglichen Matinee des Abaton-Kinos in Hamburg und zuletzt bei Veranstaltungsreihen aus Anlass des 50. Gedenktages im Jahr 2012, sowohl außerhalb Hamburgs, in Stade und in Jork, als auch im Stadtteil Wilhelmsburg. Die Zahl des Publikums beläuft sich insgesamt auf über 800 Zuschauer_innen, zumeist waren dies Zeitzeug_innen und deren Familienangehörige sowie eine interessierte Hamburger Öffentlichkeit unterschiedlicher Jahrgänge.

Die Rezeption dieses Films legt die Vermutung nahe, dass die Protagonist_innen vom Publikum als Erinnerungsakteur_innen mit Erfahrungswissen und Risikobewusstsein wahrgenommen wurden und nicht - wie in gängigen Fernsehdokumentationen - reduziert auf ihren damaligen Opferstatus. Vielmehr werden die Erlebnisse durch viele Protagonist_innen im Sinne einer Erfahrungsgemeinschaft erzählt, also „polyvokal“ vereint, dargestellt. ${ }^{567}$ Dies entspricht den eigenen Erfahrungen der Zeitzeug_innen, bspw. im innerfamiliären oder nachbarschaftlichen Austausch der Geschichten. Nicht nur die Interviewinhalte, sondern auch diese Mehrstimmigkeit wurde daher wiedererkannt.

Der Film löste bei seinen Screenings eine emotionale und zumeist nachdenkliche Stimmung aus. Er regte Teile des Publikums dazu an, nach der Vorführung über die eigenen Erfahrungen zu sprechen, also gewissermaßen selbst ergänzend Zeugnis abzulegen und die eigene Geschichte beizusteuern. ${ }^{568}$ Auch löste er im Kinosaal kontroverse Diskussionen über das Für und Wider der Elbvertiefung aus, und es wurde Kritik an dem fehlenden Erfahrungswissen heutiger Behördenvertreter_innen geäußert. Das Publikum wirkte insgesamt gut informiert und meinungsfreudig. Geprägt waren die Äußerungen sowohl von einem durchaus auch moralischen Impetus, es selbst heute ,besser zu wissen', als auch einer gewissen Frustration über die mangelnde Anerkennung ihrer Wissensbestände von behördlicher Seite.

Diese Reaktionen interpretiere ich dahingehend, dass die Zeitzeug_innen insgesamt stark in das filmische Geschehen involviert und sehr aufmerksam waren. Diese

565 Vgl. MacDougall 1997, S. 292 f.

566 Vgl. Schmidt-Lauber 2009, S. 112. Zum Begriff, Veröffentlichungsarenen` siehe Eckardt/Näser 2014, S. 273.

567 Vgl. Schmidt-Lauber 2009, S. 112.

568 Zur emotionalen Wirkungsweise von Dokumentarfilmen siehe Naber, Christina: Alles andere als nüchtern. Der aktuelle Dokumentarfilm und sein emotionales Wirkungspotenzial. In: Schick, Thomas/Ebbrecht, Tobias (Hg.): Emotion - Empathie - Figur: Spielformen der Filmwahrnehmung. Berlin 2008, S. 107-127. 
Rezeptionshaltung war davon beeinflusst, dass die Filminhalte eine für sie nach wie vor große Relevanz haben bzw. eigene, als gewichtig empfundene Erfahrungen thematisieren. ${ }^{569}$ Oder wie eingangs bereits mit Assmann formuliert, greift das, was in diesem Film als Vergangenheit „ausgewählt und aufgerufen wird, unmittelbar in die Gegenwart ein". ${ }^{570}$

Folgende Aspekte des Films Flut 1962 - Erinnern. Gedenken. Erzählen. als ein Wissensformat möchte ich daher noch einmal zusammenfassen. Dabei sei hervorgehoben, dass er als ein Repräsentationsmedium sowohl von einem ,Laien'-Publikum als auch von Fachwissenschaftler_innen rezipierbar sein soll. Anders als bei einem schriftlichen Text mit eingebetteten transkribierten Interviewausschnitten ist die Unmittelbarkeit der Erzählungen im Film gewinnbringend: Die Mehrdimensionalität gesprochener Worte bleibt in den Personen mit ihren Gesten, ihrer Mimik und ihrer Stimme im wahrsten Sinne des Wortes erhalten. ${ }^{571}$ Ein Mehrwert an rezeptionsrelevanten Eindrücken wird zudem dadurch generiert, dass die Interviewten in einer für sie repräsentativen Umgebung situiert und gefilmt sind, zumeist im privaten Wohnumfeld. Dieses Zusammenwirken von Inhalt, Aussage und Darstellung kann über das Medium Film deutlich herausgearbeitet werden, da Bild- und Tonebenen nicht nur gleichzeitig, sondern auch gleichwertig argumentieren.

Darüber hinaus entfalten Erinnerungserzählungen im Film ihre pluralen Perspektiven durch eine dramaturgische Struktur und durch filmische Mittel, wobei der Montage im Genre ,Interview-' bzw. ,Gesprächsfilm' eine wesentliche Bedeutung zukommt. Da die Protagonist_innen als eine auf Mehrstimmigkeit basierende Erzählgemeinschaft dargestellt sind, wird die Bedeutung gemeinschaftlichen Erzählens als ein zentraler Bestandteil erinnerungskultureller Praktiken betont. ${ }^{572}$ Das Medium Film eröffnet somit Zugänge, die „biografische Dimension der Motive der Erinnerung " ${ }^{\text {"573 }}$ auch an dieses Extremereignis aufzuzeigen, denn die in filmische Erzählkonventionen transferierte Stringenz und Kohärenz der Einzelaussagen unterstreicht deren sinnbildende Praxis und ihren identitätsstiftenden Charakter. Gerade die durch Dramaturgie und Montage ermöglichte kombinierte Betonung von sowohl damaligen begrenzten Handlungsspielräumen als auch von heutigen Kenntnisständen ermöglicht es, die Erzählungen als Erfolgsgeschichten zu plausibilisieren. Durch diese Art der Darstellung der „Vergegenwärtigung“ von Erinnerungen kann das Wissensformat Film im Kontext einer „neuen Erinnerungskultur“ zudem leisten, Zeitzeug_innen nicht nur abzubilden, sondern vielmehr, und das sei mit Verweis auf

\footnotetext{
569 Vgl. Cohen, Jonathan: Defining Identification. A Theoretical Look at the Identification of Audiences With Media Characters. In: Mass Communication and Society 4 (3), 2001, S. 245-264.

570 Assmann 2013, S. $205 f$.

571 Vgl. Bendix 2005.

572 „Erzählungen sind nicht nur immer an ein Publikum gerichtet, sondern sie zielen auch auf Konsens, Zustimmung und Gruppenbildung ab. Darin gründet ihre Funktion als Medium der Vergemeinschaftung, die der Vorstellung von Kulturen als Erzählgemeinschaften zugrunde liegt." Nünning 2013, S. 43.

573 Thießen 2007, S. 19.
} 
Assmann hervorgehoben, ,ihre Eigenart [zu] markieren, ihr Selbstbewusstsein [zu] stärken und Orientierung für die Zukunft" zu geben. ${ }^{574}$ Dies erfolgt insbesondere dadurch, dass der Wert und Eigensinn der Erzählungen für ein Publikum dargestellt, respektive nachvollziehbar gemacht wird. Somit versteht sich Flut 1962 - Erinnern. Gedenken. Erzählen. auch als das Ergebnis einer Vermittlungsarbeit.

Vor diesem Hintergrund lässt sich die entwickelte Dramaturgie hier ein weiteres Mal begründen, da sie darauf abzielt, die heutige aktive Erzählhaltung der Protagonist_innen zu betonen. Dies ist durch die gezeigten Erinnerungspraktiken im ersten Filmteil sowie durch die in szenische Momente eingebetteten Reflexionen im dritten Filmteil umgesetzt. Neben meiner bereits angeführten Erzählabsichten - inklusive der Adressierung eines Publikums mit dem Ziel, einen gemeinsamen Erfahrungsraum herzustellen - sind dies performative Repräsentationsweisen seitens der Befragten, die ihre audiovisuelle Entsprechung besonders durch das Medium Film erhalten können.

Eine Besonderheit der hier analysierten Erinnerungserzählungen ist eine bildreiche, an das Drama angelehnte, Plotstruktur. Sie verläuft in ihrem strukturellen Aufbau analog zu den Sehgewohnheiten filmischer Darstellungskonventionen. Daher können insbesondere die persönlichen Masternarrationen gut in das Wissensformat Film transformiert werden: Sie verfügen über einen Anfang und ein Ende sowie einen Spannungsaufbau und Wendepunkte. Somit begünstigt diese Nähe zu fiktionalen Erzählweisen ein filmisches „Explanation by Emplotment“. ${ }^{575}$ Dieser Konstruktionscharakter der Erinnerungserzählungen wird im Film durch zeitgenössisches Foto- und Filmmaterial zusätzlich verstärkt. ${ }^{576}$

Als Repräsentationsmedium bietet Flut 1962 - Erinnern. Gedenken. Erzählen. somit besondere Möglichkeiten, wissenschaftliche Ergebnisse über das untersuchte Feld hinaus öffentlich zu präsentieren. Der selbst produzierte Dokumentarfilm wird hier zu einem Wissensformat, das Ergebnisse einer Erzählforschung als Bewusstseinsanalyse zu dem oben zitierten "image-and-sequence-based anthropological thought“ zusammenführt. ${ }^{577}$ Dabei ist es unerheblich, dass es sich hier um einen auf Interviewaussagen basierenden Film handelt, in dem nur wenige szenische Sequenzen enthalten und zumeist ähnliche Einstellungsgrößen der Talking Heads zu sehen sind. Dies könnte den Eindruck einer wenig innovativen filmischen Gestaltung erwecken, wie die hier dargelegten innerfachlichen kritischen Diskurse womöglich nahelegen. ${ }^{578}$ Vielmehr konnten durch die Dramaturgie und die gewählten filmischen Mittel - insbesondere durch eine Montage, umgesetzt nicht „anhand der Bilder, sondern anhand der Worte ${ }^{\text {" } 579}$ - wesentliche Untersuchungsergebnisse in das Medium Film transformiert werden, nämlich die für die Erinnerungserzählungen relevanten Foto-

\footnotetext{
574 Vgl. Assmann 2013, S. 205.

575 White 1975, S. 7. Siehe hierzu ausführlich Kapitel 2.5.

576 Weiterführend zur Konstruktion des Erzählens siehe Lehmann 2007, S. $50 \mathrm{ff}$.

577 Vgl. MacDougall 1997, S. 292.

578 Siehe hierzu Kapitel 2.6.1 und Kapitel 4.2.

579 Vgl. Wilbers 2001, S. 281.
} 
grafien und Fotoalben sowie die strukturellen und inhaltlichen Besonderheiten der persönlichen Masternarrationen. Darüber hinaus können diese Geschichten im Kontext erinnerungskultureller Praktiken als Artikulationen von Erfahrungswissen und - im Hinblick auf mögliche zukünftige Gefahren durch Sturmfluten bzw. Hochwasser - eines Risikobewusstseins interpretiert werden. Diese Ergebnisse, erhoben aus der Verbindung von Erzählforschung und ethnografisch-filmischer Methode, stellen somit zudem einen erfahrungsorientierten Beitrag zu aktuellen transdisziplinären Diskursen im Kontext von Resilienz und Vulnerabilität dar. 



\section{Literatur- und Quellenverzeichnis}

\section{Sekundärliteratur}

Alexander, Jeffrey C.: Towards a Theory of Cultural Trauma. In: Ders. u. a. (Hg.):

Cultural Trauma and Collective Identity. Berkeley/London 2004, S. 1-30.

Allemeyer, Marie Luisa: Nordsee ist Mordsee. Rezension. In: sehepunkte 6 (2006),

Nr. 7/8: http://www.sehepunkte.de/2006/07/9339.html (aufgerufen am

11.11.2019)

Assmann, Aleida: Erinnerungsräume. Formen und Wandlungen des kulturellen Gedächtnisses. München 1999.

Assmann, Aleida: Gedächtnis als Leitbegriff der Kulturwissenschaften. In: Musner, Lutz/Wunberg, Gotthart (Hg.): Kulturwissenschaften. Forschung - Praxis - Positionen. Freiburg i. Br. 2003, S. 27-47.

Assmann, Aleida: Jahrestage - Denkmäler in der Zeit. In: Münch, Paul (Hg.):

Jubiläum, Jubiläum ... Zur Geschichte öffentlicher und privater Erinnerung. Essen 2005, S. 305-314.

Assmann, Aleida: Das neue Unbehagen an der Erinnerungskultur. München 2013.

Balkenhol, Thomas: Pflicht und Kür der Dokumentarfilm-Montage. „24 Mal Wahrheit in der Sekunde“ (Godard) - Der Umgang mit der Realität in der Montage von Dokumentarfilmen. In: Beller, Hans (Hg.): Handbuch der Filmmontage. München 1993, S. 123-143.

Ballhaus, Edmund: Der volkskundliche Film. Ein Beitrag zur Theorieund Methodendiskussion. In: Hessische Blätter für Volks- und Kulturforschung NF 21 (1987), S. 108-130.

Ballhaus, Edmund: Film und Feldforschung. In: Ders./Engelbrecht, Beate (Hg.): Der ethnographische Film. Eine Einführung in Methoden und Praxis. Berlin 1995, S. 13-46 (abgekürzt: 1995a).

Ballhaus, Edmund: Das Dilemma als Chance. Der kulturwissenschaftliche Film im Prozeß der Feldforschung. In: Lipp, Carola (Hg.): Medien popularer Kultur. Erzählung, Bild und Objekt in der volkskundlichen Forschung. Frankfurt a. M./New York 1995, S. 417-432 (abgekürzt: 1995b).

Ballhaus, Edmund (Hg.): Kulturwissenschaft, Film und Öffentlichkeit. Münster 2001.

Ballhaus, Edmund: Rede und Antwort. Antwort oder Rede? Interviewformen im kulturwissenschaftlichen Film. In: Wossidlo, Joachim/Roters, Ulrich (Hg.): Interview und Film. Volkskundliche und Ethnologische Ansätze zu Methodik und Analyse. Münster u. a. 2003, S. 11-47. 
Ballhaus, Edmund: Erkenntnis als Inszenierung. Inszenierung als Erkenntnis. In: Simon, Michael u. a. (Hg.): Bilder. Bücher. Bytes. Zur Medialität des Alltags. 36. Kongress der Deutschen Gesellschaft für Volkskunde in Mainz vom 23. bis 26. September 2007. Münster u. a. 2009, S. 164-172.

Ballhaus, Edmund (Hg.): Dokumentarfilm: Schulen - Projekte - Konzepte. Berlin 2013 (abgekürzt: 2013a).

Ballhaus, Edmund: Kulturwissenschaftliche Erkenntnis als dokumentarische Inszenierung. Dokumentarfilm in Göttingen. In: Ders. (Hg.):

Dokumentarfilm: Schulen - Projekte - Konzepte. Berlin 2013, S. 234-264 (abgekürzt: 2013b).

Bankoff, Greg: Cultures of Disaster. Society and natural hazards in the Philippines. London 2003.

Bankoff, Greg: The Historical Geography of Disaster. ,Vulnerability' and ,Local Knowledge' in Western Discourse. In: Ders./Frerks, Georg/Hilhorst, Dorothea (Hg.): Mapping Vulnerability. Disasters, Development and People. London/Sterling 2004, S. 25-36.

Bankoff, Greg: Comparing Vulnerabilities. Towards Charting a Historical Trajectory of Disasters. In: Historical Social Research 32 (3), 2007, S. 103-114.

Bartels, Marie: Katastrophen und Kausalität. In: Hempel, Leon/Dies./Markwart, Thomas (Hg.): Aufbruch ins Unversicherbare. Zum Katastrophendiskurs der Gegenwart. Bielefeld 2013, S. 193-207.

Beck, Stefan: Die Bedeutung der Materialität der Alltagsdinge. Anmerkungen zu den Chancen einer wissenschaftstheoretisch informierten Integration von Symbol- und Sachforschung. In: Brednich, Rolf Wilhelm/Schmitt, Heinz (Hg.): Symbole. Zur Bedeutung der Zeichen in der Kultur. 30. Deutscher Volkskundekongreß in Karlsruhe vom 25. bis 29. September 1995.

Münster u. a. 1997, S. 175-186.

Beck, Ulrich: Risikogesellschaft. Auf dem Weg in eine andere Moderne. Frankfurt a. M. 1986.

Bendix, Regina: Reflections on Earthquake Narratives. In: Western Folklore 49 (1),1990, S. 331-347.

Bendix, Regina: Zwischen Chaos und Kultur. Zur Ethnographie des Erzählens im ausgehenden 20. Jahrhundert. In: Zeitschrift für Volkskunde 92 (1), 1996, S. 169-184.

Bendix, Regina: Die Stimme: Eine Spurensuche. In: Hengartner, Thomas/ Schmidt-Lauber, Brigitta (Hg.): Leben - Erzählen. Beiträge zur Erzählund Biographieforschung. Festschrift für Albrecht Lehmann. Berlin/ Hamburg 2005, S. 71-95. 
Berek, Mathias: Kollektives Gedächtnis und die gesellschaftliche Konstruktion der Wirklichkeit. Eine Theorie der Erinnerungskulturen. Wiesbaden 2009.

Böhl, Michael: Entwicklung des ethnographischen Films. Die filmische

Dokumentation als ethnographisches Forschungs- und universitäres

Untersuchungsmittel in Europa. Göttingen 1985.

Böhme, Gernot: Atmosphäre. Essays zur neuen Ästhetik. Frankfurt a. M. 1995.

Bohle, Hans-Georg: Leben mit Risiko - Resilience als neues Paradigma für die

Risikowelten von morgen. In: Felgentreff, Carsten/Glade, Thomas (Hg.):

Naturrisiken und Sozialkatastrophen. Heidelberg 2008, S. 435-441.

Bohnsack, Ralf: Qualitative Bild- und Videointerpretation. Die dokumentarische

Methode. 2., durchgesehene und aktualisierte Auflage. Opladen/

Farmington Hills 2011.

Bonß, Wolfgang: (Un-)Sicherheit in der Moderne. In: Zoche, Peter/Kaufmann,

Stefan/Haverkamp, Rita (Hg.): Zivile Sicherheit. Gesellschaftliche

Dimensionen gegenwärtiger Sicherheitspolitiken. Bielefeld 2010,

S. 43-71.

Bonß, Wolfgang: Karriere und sozialwissenschaftliche Potenziale des

Resilienzbegriffs. In: Endreß, Martin/Maurer, Andrea (Hg.): Resilienz im

Sozialen. Wiesbaden 2015, S. 15-31.

Borst, Arno: Das Erdbeben von 1348. Ein historischer Beitrag zur

Katastrophenforschung. In: Historische Zeitschrift 233 (3),1981,

S. 529-569.

Bourdieu, Pierre u. a. (Hg.): Eine illegitime Kunst. Die sozialen Gebrauchsweisen der Fotografie. Frankfurt a. M. 1981.

Bracke, Sarah: Bouncing Back. Vulnerability and Resistance in Times of Resilience. In: Butler, Judith/Gambetti, Zeynep/Sabsay, Leticia (Hg.): Vulnerability in Resistance. London 2016, S. 52-75.

Brednich, Rolf Wilhelm: Vorwort. In: Ders./Schneider, Annette/Werner, Ute (Hg.): Natur - Kultur. Volkskundliche Perspektiven auf Mensch und Umwelt. 32. Kongreß der Deutschen Gesellschaft für Volkskunde in Halle vom 27.9. bis 1.10.1999. Münster u. a. 2001, S. XI-XII.

Cohen, Jonathan: Defining Identification. A Theoretical Look at the Identification of Audiences With Media Characters. In: Mass Communication and Society 4 (3), 2001, S. 245-264.

Christmann, Gabriela B./Balgar, Karsten/Mahlkow, Nicole: Zur sozialwissenschaftlichen Konzeption von Vulnerabilität und Resilienz. Konzeptionelle Überlegungen und empirische Betrachtungen am Beispiel von Städten. In: Endreß, Martin/Maurer, Andrea (Hg.): Resilienz des Sozialen. Wiesbaden 2015, S. 123-149. 
Douglas, Mary: Risk and Blame. Essays in Cultural Theory. London/New York 1992.

Eckardt, Sandra/Näser, Torsten: Ethnografisches Filmen. In: Bischoff, Christine/ Oehme-Jüngling, Karoline/Leimgruber, Walter (Hg.): Methoden der Kulturanthropologie. Bern 2014, S. 273-290.

Edwards, Elisabeth: Photographs as Objects of Memory. In: Kwint, Marius/ Breward, Christopher/Aynsley, Jeremy (Hg.): Material Memories. Oxford/ New York 1999, S. 221-236.

Engelbrecht, Beate: Film als Methode in der Ethnologie. In: Ballhaus, Edmund/ Dies. (Hg.): Der ethnographische Film. Eine Einführung in Methoden und Praxis. Berlin 1995, S. 143-186.

Engelbrecht, Beate: For Whom Do We Produce? In: Crawford, Peter Ian (Hg.): The Construction of the Viewer. Aarhus 1996, S. 163-176.

Engels, Jens Ivo: Vom Subjekt zum Objekt. Naturbild und Naturkatastrophen in der Geschichte der Bundesrepublik Deutschland. In: Groh, Dieter/ Kempe, Michael/Mauelshagen, Franz (Hg.): Naturkatastrophen. Beiträge zu ihrer Deutung, Wahrnehmung und Darstellung in Text und Bild von der Antike bis ins 20. Jahrhundert. Tübingen 2003, S. 119-142.

Eßler, Henrik: (Rezension zu) Felix Mauch: Erinnerungsfluten. Das Sturmfluthochwasser von 1962 im Gedächtnis der Stadt Hamburg. München/Hamburg 2015. In: Zeitschrift für Geschichtswissenschaft. 64. Jg. (2016) 10, S. 917-919.

Felgentreff, Carsten/Glade, Thomas: Naturereignisse sind unausweichlich, Katastrophen nicht?! In: Dies. (Hg.): Naturrisiken und Sozialkatastrophen. Heidelberg 2008, S. 443-448.

Fellmer, Mareike: Bürgerschaftliches Engagement und Sturmfluten. Ausprägungen und Einflussfaktoren am Beispiel des Flussgebietes der Tideelbe. Detmold 2014.

Fischer, Norbert: Wassernot und Marschgesellschaft. Zur Geschichte der Deiche in Kehdingen. Stade 2003.

Fischer, Norbert: Einführung. In: Fiedler, Gudrun (Hg. im Auftrag des Stader Geschichts- und Heimatvereins): Stader Jahrbuch 2011. 50 Jahre Sturmflut 1962. Ereignis - Geschichte - Gedenken. Stade 2011, S. 13-15 (abgekürzt: 2011a).

Fischer, Norbert: Maritime Gedächtniskultur an der Nordseeküste. Adaptionen der Katastrophe. In: Fischer, Ludwig/Reise, Karsten (Hg.): Küstenmentalität und Klimawandel. Küstenwandel als kulturelle und soziale Herausforderung. München 2011, S. 77-95 (abgekürzt: 2011b).

Fischer, Norbert: Der wilde und der gezähmte Fluss - Zur Geschichte der Deiche an der Oste. Stade 2011 (abgekürzt: 2011c). 
Fischer, Norbert: Gedächtnislandschaften in Geschichte und Gegenwart.

Kulturwissenschaftliche Studien. Wiesbaden 2016.

Frank, Arthur W.: Letting Stories Breathe. A socio-narratology. Chicago/London 2010.

Frank, Saskia: Zeppelin-Ereignisse. Technikkatastrophen im medialen Prozess. Marburg 2008.

Gerhold, Lars: Risikobewusstsein. Sicherheit als Konstrukt gesellschaftlicher

Wahrnehmung. In: Daase, Christopher/Offermann, Philipp/Rauer,

Valentin (Hg.): Sicherheitskultur. Soziale und politische Praktiken der

Gefahrenabwehr. Frankfurt a. M./New York 2012, S. 341-356.

Gerndt, Helge: Kulturvermittlung. Modellüberlegungen zur Analyse eines

Problemkomplexes am Beispiel des Atomunglücks von Tschernobyl. In:

Zeitschrift für Volkskunde 86 (1), 1990, S. 1-13.

Gerndt, Helge: Mit Bildern erzählen. In: Brednich, Rolf Wilhelm (Hg.):

Erzählkultur. Beiträge zur kulturwissenschaftlichen Erzählforschung.

Hans-Jörg Uther zum 65. Geburtstag. Berlin/New York 2009, S. 309-325.

Gönnert, Gabriele: Sturmfluten in der Elbe - Das Hochwasser- und

Bemessungskonzept für Hamburg. In: Ratter, Beate M. W. (Hg.): Küste

und Klima. Hamburger Symposium Geographie, Band 1. Hamburg 2009, S. 23-33.

Groh, Dieter/Kempe, Michael/Mauelshagen, Franz: Einleitung.

Naturkatastrophen - wahrgenommen, gedeutet, dargestellt. In: Dies.

(Hg.): Naturkatastrophen. Beiträge zu ihrer Deutung, Wahrnehmung und Darstellung in Text und Bild von der Antike bis ins 20. Jahrhundert.

Tübingen 2003, S. 11-33.

Hägele, Ulrich: Visual Folklore. Zur Rezeption und Methodik der Fotografie In:

Göttsch, Silke/Lehmann, Albrecht (Hg.): Methoden der Volkskunde.

Berlin 2001, S. 277-300.

Hägele, Ulrich: Visuelle Kultur? Thesen zum erweiterten Fachverständnis

bildmedialer Forschung. In: Gerndt, Helge/Haibl, Michaela (Hg.):

Der Bilderalltag. Perspektiven einer volkskundlichen Bildwissenschaft.

Münster u. a. 2005, S. 375-388.

Hänel, Dagmar: Anmerkungen zum gefilmten Interview in der Volkskunde.

In: Wossidlo, Joachim/Roters, Ulrich (Hg.): Interview und Film.

Volkskundliche und Ethnologische Ansätze zu Methodik und Analyse.

Münster u. a. 2003, S. 107-118.

Halbwachs, Maurice: Das Gedächtnis und seine sozialen Bedingungen.

Frankfurt a. M. 1985 (1925) (abgekürzt: 1985a).

Halbwachs, Maurice: Das kollektive Gedächtnis. Frankfurt a. M. 1985 (1939)

(abgekürzt: 1985b). 
Hattendorf, Manfred: Dokumentarfilm und Authentizität. Ästhetik und Pragmatik einer Gattung. Konstanz 1994.

Hempel, Leon/Markwart, Thomas: Einleitung. Ein Streit über die Katastrophe. In: Hempel, Leon/Bartels, Marie/Markwart, Thomas (Hg.): Aufbruch ins Unversicherbare. Zum Katastrophendiskurs der Gegenwart. Bielefeld 2013, S. 7-27.

Henley, Paul: Are you happy? Interviews, 'conversations' and 'talking heads' as methods for gathering oral testimony in ethnographic documentary. In: Wossidlo, Joachim/Roters, Ulrich (Hg.): Interview und Film. Volkskundliche und Ethnologische Ansätze zu Methodik und Analyse. Münster u. a. 2003, S. 51-67.

Herlyn, Gerrit: Computer im Alltag - Computer als Alltag. Erzählstrategien und biographische Deutungen im Veralltäglichungsprozess von Technik. Hamburg 2008.

Herlyn, Gerrit: Systematik oder Intuition? Zur kulturanalytischen Praxis der Auswertung qualitativer Interviews. In: Johler, Reinhard u. a. (Hg.): Kultur_Kultur. Denken. Forschen. Darstellen. 38. Kongress der Deutschen Gesellschaft für Volkskunde in Tübingen vom 21.-24. September 2011. Münster u. a. 2013, S. 485-491.

Heßler, Martina/Kehrt, Christian: Einleitung: Die Hamburger Sturmflut. Betrachtungen aus zeit-, technik- und umwelthistorischer Perspektive. In: Dies. (Hg.): Die Hamburger Sturmflut von 1962. Risikobewusstsein und Katastrophenschutz aus zeit-, technik- und umweltgeschichtlicher Perspektive. Göttingen/Bristol 2014, S. 9-34.

Hickethier, Knut: Film- und Fernsehanalyse. 4., aktualisierte und erweiterte Auflage. Stuttgart 2007.

Hinrichsen, Jan: Evidenz und Unsicherheit. Zur materiellen Kultur der Katastrophe. In: Braun, Karl/Dieterich, Claus-Marco/Treiber, Angela (Hg.): Materialisierung von Kultur. Diskurse Dinge Praktiken. Würzburg 2015, S. 444-451.

Hinrichsen, Jan/Johler, Reinhard/Ratt, Sandro: Katastrophen. Vom kulturellen Umgang mit (außer)alltäglichen Bedrohungen. In: Frie, Ewald/Meier, Mischa (Hg.): Aufruhr - Katastrophe - Konkurrenz - Zerfall. Bedrohte Ordnungen als Thema der Kulturwissenschaften. Tübingen 2014, S. 61-82.

Hißnauer, Christian: Fernsehdokumentarismus. Theoretische Näherungen, pragmatische Abgrenzungen, begriffliche Klärungen. Konstanz 2011.

Hugger, Paul: Elemente einer Ethnologie der Katastrophe in der Schweiz. In: Zeitschrift für Volkskunde 86 (1), 1990, S. 25-36. 
Imhof, Kurt: Katastrophenkommunikation in der Moderne. In: Pfister, Christian/ Summermatter, Stephanie (Hg.): Katastrophen und ihre Bewältigung. Perspektiven und Positionen. Bern/Stuttgart/Wien 2004, S. 145-163. Jakubowski-Tiessen, Manfred/Lehmann, Hartmut: Religion in Katastrophenzeiten: Zur Einführung. In: Dies. (Hg.): Um Himmels Willen. Religion in Katastrophenzeiten. Göttingen 2003, S. 7-13. Jank, Anna: Erzählen über Katastrophen. 8. Tagung der Kommission für Erzählforschung in der Deutschen Gesellschaft für Volkskunde. Gösing an der Mariazellerbahn, 3. bis. 6. September 2014. In: Österreichische Zeitschrift für Volkskunde 117 (3/4), 2014, S. 300-305.

Jeismann, Karl-Ernst: Geschichtsbewußtsein. In: Bergmann, Klaus u. a. (Hg.): Handbuch der Geschichtsdidaktik. 4. Auflage. Seelze 1992, S. 40-43. Johler, Reinhard: Die Kultur der Katastrophe und die Materialisierung von Kultur. In: Braun, Karl/Dieterich, Claus-Marco/Treiber, Angela (Hg.): Materialisierung von Kultur. Diskurse Dinge Praktiken. Würzburg 2015, S. 438-443.

Jureit, Ulrike: Überlebensdiskurse. Zur Methodik wissenschaftlicher Analyse von Interviews mit Überlebenden der Konzentrations- und Vernichtungslager. Hamburg 1999.

Kapucu, Naim/Hawkins, Christopher V./Rivera, Fernando: Disaster Resiliency. Interdisciplinary Perspectives. In: Dies. (Hg.): Disaster Resiliency. Interdisciplinary Perspectives. New York/London 2013, S. 1-14. Kaufmann, Stefan: Resilienz als ,Boundary Object'. In: Daase, Christopher/ Offermann, Philipp/Rauer, Valentin (Hg.): Sicherheitskultur. Soziale und politische Praktiken der Gefahrenabwehr. Frankfurt a. M./New York 2012, S. 109-131.

Kaufmann, Stefan/Blum, Sabine: Vulnerabilität und Resilienz. Zum Wandern von Ideen in der Umwelt- und Sicherheitsdiskussion. In: Detten, Roderich von/Faber, Fenn/Bemmann, Martin (Hg.): Unberechenbare Umwelt. Wiesbaden 2013, S. 91-120.

Kawan, Christine Shojaei: Tagungsbericht. 8. Tagung der Kommission für Erzählforschung in der dgv. Gösling, 3.9.-6.9.2014. In: Fabula 55 (1/2), 2014, S. 301-306.

Keifenheim, Barbara: Der Einsatz von Film und Video. In: Beer, Bettina (Hg.): Methoden und Techniken der Feldforschung. Berlin 2003, S. 249-263.

Keller, Reiner: Zur Chronik angekündigter Katastrophen. Die Umweltkrise als Dauerevent. In: Gebhardt, Winfried/Hitzler, Ronald/Pfadenhauer, Michaela (Hg.): Events. Soziologie des Außergewöhnlichen. Opladen 2000, S. 263-285. 
Kempe, Michael: 'Mind the Next Flood!' Memories of Natural Disasters in Northern Germany from the Sixteenth Century to the Present. In: The Medieval History Journal 10 (1/2), 2007, S. 327-354.

Ketterer, Werner/Spada, Hans: Der Mensch als Betroffener und Verursacher von Naturkatastrophen. In: Plate, Erich J. (Hg.): Naturkatastrophen und Katastrophenvorbeugung. Bericht des Wissenschaftlichen Beirats der DFG für das Deutsche Komitee für die „International Decade for Natural Disaster Reduction“ (IDNDR). Weinheim u. a. 1993, S. 73-107.

Kiener, Wilma: Die Kunst des Erzählens. Narrativität in dokumentarischen und ethnographischen Filmen. Konstanz 1999.

Kirshenblatt-Gimblett, Barbara: Objects of Memory. Material Culture as Life Review. In: Oring, Elliot (Hg.): Folk Groups and Folklore Genres. A Reader. Logan 1989, S. 329-338.

Knieling, Jörg/Fellmer, Mareike: Climate Adaption in Metropolis Hamburg: Paradigm Shift in Urban Planing and Water Management towards 'Living with Water'? In: Schmidt-Thomé, Philipp/Klein, Johannes (Hg.): Climate Change Adaptation in Practice: From Strategy Development to Implementation. Chichester u. a. 2013, S. 83-93.

Knieling, Jörg/Schaerffer, Mareike/Tressl, Stephan: Klimawandel und Raumplanung. Flächen- und Risikomanagement überschwemmungsgefährdeter Gebiete am Beispiel der Hamburger Elbinsel. Hamburg 2009.

Knoblauch, Hubert: Topik und Soziologie. Von der sozialen und kommunikativen Topik. In: Schirren, Thomas/Ueding, Gert (Hg.): Topik und Rhetorik. Ein interdisziplinäres Symposium. Tübingen 2000, S. 651-667.

Köstlin, Konrad: Photographierte Erinnerung? Bemerkungen zur Erinnerung im Zeitalter ihrer technischen Reduzierbarkeit. In: Brunhold-Bigler, Ursula/ Bausinger, Hermann (Hg.): Hören Sagen Lesen Lernen. Bausteine zu einer Geschichte der kommunikativen Kultur. Festschrift für Rudolf Schenda zum 65. Geburtstag. Bern 1995, S. 395-410.

Kofler, Alexandra: Erzählen über Liebe. Die Konstruktion von Identität in autobiografischen Interviews. Frankfurt a. M./New York 2012.

Koselleck, Reinhardt: Formen und Traditionen des negativen Gedächtnisses. In: Knigge, Volkhard/Frei, Norbert (Hg.): Verbrechen erinnern. Die Auseinandersetzung mit Holocaust und Völkermord. München 2002, S. 21-32.

Leh, Almut: Forschungsethische Probleme in der Zeitzeugenforschung. In: BIOS 13 (1), 2000, S. 64-76.

Lehmann, Albrecht: Erzählen eigener Erlebnisse im Alltag. Tatbestände, Situationen, Funktionen. In: Zeitschrift für Volkskunde 74 (2), 1978, S. 198-215. 
Lehmann, Albrecht: Rechtfertigungsgeschichten. Über die Funktion des Erzählens eigener Erlebnisse im Alltag. In: Fabula 21 (1), 1980, S. 56-69.

Lehmann, Albrecht: Erzählstruktur und Lebenslauf. Autobiographische Untersuchungen. Frankfurt a. M./New York 1983.

Lehmann, Albrecht: Der Schicksalsvergleich. Eine Gattung des Erzählens und eine Methode des Erinnerns. In: Bönisch-Brednich, Brigitte/Brednich, Rolf Wilhelm/Gerndt, Helge (Hg.): Erinnern und Vergessen. Vorträge des 27. Deutschen Volkskundekongresses Göttingen 1989. Göttingen 1991, S. 197-207.

Lehmann, Albrecht: Aspekte populären Landschaftsbewußtseins. In: Siemann, Wolfram (Hg.): Umweltgeschichte. Themen und Perspektiven. München 2003, S. 147-164.

Lehmann, Albrecht: Reden über Erfahrung. Kulturwissenschaftliche Bewusstseinsanalyse des Erzählens. Berlin 2007.

Lehmann, Albrecht: Homo narrans - Individuelle und kollektive Dimensionen des Erzählens. In: Brednich, Rolf Wilhelm (Hg.): Erzählkultur. Beiträge zur kulturwissenschaftlichen Erzählforschung. Hans-Jörg Uther zum 65. Geburtstag. Berlin/New York 2009, S. 60-70.

Leikauf, Roland: „Welcome to My Bunker“ - Vietnamkriegserfahrungen im Internet. Bielefeld 2016.

Leimgruber, Walter: Ethnografischer Film: (un)geliebtes Stiefkind der Kulturwissenschaft. In: Röösli, Lisa/Risi, Marius: Lebensbilder Bilderwandel. Zwei ethnografische Filmprojekte im Alpenraum. Münster 2010, S. 9-24.

Leimgruber, Walter/Andris, Silke/Bischoff, Christine: Visuelle Anthropologie: Bilder machen, analysieren, deuten und präsentieren. In: Hess, Sabine/ Moser, Johannes/Schwertl, Maria (Hg.): Europäisch-ethnologisches Forschen. Neue Methoden und Konzepte. Berlin 2013, S. 247-281. Lindner, Rolf: Ohne Gewähr. Zur Kulturanalyse des Informanten. In: Jeggle, Utz (Hg.): Feldforschung. Qualitative Methoden in der Kulturanalyse. Tübingen 1984, S. 59-71.

Löffler, Klara: Zurechtgerückt. Der Zweite Weltkrieg als biographischer Stoff. Berlin 1999.

Lucius-Hoene, Gabriele/Deppermann, Arnulf: Rekonstruktion narrativer Identität. Ein Arbeitsbuch zur Analyse narrativer Interviews. 2. Auflage. Wiesbaden 2004.

MacDougall, David: Ein nichtpriviligierter Kamerastil. In: Friedrich, Margarete (Hg.): Die Fremden sehen. München 1984, S. 73-83. 
MacDougall, David: The Visual in Anthropology. In. Banks, Marcus/Morphy, Howard (Hg.): Rethinking Visual Anthropology. New Haven 1997, S. 276-295.

MacDougall, David: Visual Anthropology and the Ways of Knowing. In: Ders.: Transcultural Cinema. Princeton 1998, S. 61-92.

Markert, Margret: Eine Insel wird Industriegebiet - Portrait des Reiherstiegviertels. In: Geschichtswerkstatt Wilhelmsburg \& Hafen/Museum Elbinsel Wilhelmsburg (Hg.): Wilhelmsburg. Hamburgs große Elbinsel. Hamburg 2008, S. 41-58.

Mathys, Nora: Fotofreundschaften. Visualisierungen von Nähe und Gemeinschaft in privaten Fotoalben aus der Schweiz 1900-1950. Baden 2013.

Mauch, Christof: Introduction. In: Ders./Pfister, Christian (Hg.): Natural Disasters, Cultural Responses. Case Studies towards a Global Environmental History. Lanham u. a. 2009, S. 1-16.

Mauch, Felix: Die Natur der Katastrophe. Ein umwelthistorischer Rückblick auf die Hamburger Sturmflut. In: Heßler, Martina/Kehrt, Christian (Hg.): Die Hamburger Sturmflut von 1962. Risikobewusstsein und Katastrophenschutz aus zeit-, technik- und umweltgeschichtlicher Perspektive. Göttingen/Bristol 2014, S. 129-148.

Mauch, Felix: Erinnerungsfluten. Das Sturmfluthochwasser von 1962 im Gedächtnis der Stadt Hamburg. München/Hamburg 2015.

Meyer, Carla/Patzel-Mattern, Katja/Schenk, Gerrit Jasper: Krisengeschichte(n). „Krise“ als Leitbegriff und Erzählmuster in kulturwissenschaftlicher Perspektive - Eine Einführung. In: Dies. (Hg.): Krisengeschichte(n). „Krise“ als Leitbegriff und Erzählmuster in kulturwissenschaftlicher Perspektive. Stuttgart 2013, S. 9-23.

Meyer, Silke: Was heißt Erzählen? Die Narrationsanalyse als hermeneutische Methode der Europäischen Ethnologie. In: Zeitschrift für Volkskunde 110 (2), 2014, S. 243-267.

Naber, Christina: Alles andere als nüchtern. Der aktuelle Dokumentarfilm und sein emotionales Wirkungspotenzial. In: Schick, Thomas/Ebbrecht, Tobias (Hg.): Emotion - Empathie - Figur: Spielformen der Filmwahrnehmung. Berlin 2008, S. 107-127.

Näser, Torsten: Film und Text. Ethnografische Wissensformate im Diskursvergleich. Berlin 2014.

Näser, Torsten: Die Rede vom Stiefkind. Sprachliche Performativität des ethnografischen Films. In: kulturen (2), 2016, S. 37-54.

Netenjakob, Egon: Eberhard Fechner. Lebensläufe dieses Jahrhunderts im Film. Weinheim/Berlin 1989. 
Nolte, Paul: Von Glück und Streit, Lernen und Stabilität. Historiografische Meistererzählungen deutscher Demokratie. In: Hertfelder, Thomas/ Lappenküper, Ulrich/Lillteicher, Jürgen (Hg.): Erinnern an Demokratie in Deutschland. Demokratiegeschichte in Museen und Erinnerungsstätten der Bundesrepublik. Göttingen 2016, S. 121-137.

Nünning, Ansgar: Grundzüge einer Narratologie der Krise. Wie aus einer Situation ein Plot und eine Krise (konstruiert) werden. In: Grunwald, Henning/Pfister, Manfred (Hg.): Krisis! Krisenszenarien, Diagnosen und Diskursstrategien. München 2007, S. 48-71.

Nünning, Ansgar: Wie Erzählungen Kulturen erzeugen. Prämissen, Konzepte und Perspektiven für eine kulturwissenschaftliche Narratologie. In: Strohmaier, Alexandra (Hg.): Kultur - Wissen - Narration. Perspektiven transdisziplinärer Erzählforschung für die Kulturwissenschaften. Bielefeld 2013, S. 15-53.

Oliver-Smith, Anthony/Hoffman, Susanna M.: Introduction. Why Anthropologists Should Study Disasters. In: Dies. (Hg.): Catastrophe \& Culture. The Anthropology of Disaster. Oxford 2002, S. 3-22.

Overdick, Thomas: Martin Rosswog: ein visueller Ethnograph des ländlichen Europas. In: Simon, Michael u. a. (Hg.): Bilder. Bücher. Bytes. Zur Medialität des Alltags. 36. Kongress der Deutschen Gesellschaft für Volkskunde in Mainz vom 23. bis 26. September 2007. Münster u. a. 2009, S. 173-182.

Paech, Frauke: „Solange der Michel steht“. Lebensgeschichtliche Erzählungen als Thema für den volkskundlich-kulturwissenschaftlichen Film. In: VOKUS 11 (2), 2001, S. 4-43.

Paech, Frauke: „Die ganzen menschlichen Geschichten“ - Die Hamburger Sturmflut von 1962 im Bewusstsein der Wilhelmsburger Bevölkerung. In: Geschichtswerkstatt Wilhelmsburg \& Hafen/Museum Elbinsel Wilhelmsburg (Hg.): Wilhelmsburg. Hamburgs große Elbinsel. Hamburg 2008, S. 161-173.

Paech, Frauke: Eine Naturkatastrophe gendern?! Erzählungen über die Hamburger Sturmflut von 1962. In: Ariadne. Forum für Frauen- und Geschlechtergeschichte 64, 2013, S. 58-65.

Pagenstecher, Cord: Zwischen Tourismuswerbung und Autobiografie. Erzählkulturen in Urlaubsalben. In: Spode, Hasso/Ziehe, Irene (Hg.): Gebuchte Gefühle. Tourismus zwischen Verortung und Entgrenzung. München/Wien 2005, S. 82-91. 
Perren-Klingler, Gisela: Katastrophen: Betroffenheit von Einzelnen und Gemeinschaften. Über die Rolle der Narrative beim Umgang mit psychischen Folgen. In: Pfister, Christian/Summermatter, Stephanie (Hg.): Katastrophen und ihre Bewältigung. Perspektiven und Positionen. Bern/Stuttgart/Wien 2004. S. 217-230.

Pethes, Nicolas: Kulturwissenschaftliche Gedächtnistheorien zur Einführung. Hamburg 2008.

Pfister, Christian: Naturkatastrophen und Naturgefahren in geschichtlicher Perspektive. Ein Einstieg. In: Ders. (Hg.): Am Tag danach - Zur Bewältigung von Naturkatastrophen in der Schweiz 1500-2000. Bern/ Stuttgart/Wien 2002, S. 11-25.

Pfister, Christian: „The Monster Swallows You“. Disaster Memory and Risk Culture in Western Europe, 1500-2000. In: RCC Perspectives (1) 2011, S. 1-23.

Plato, Alexander von: Medialität und Erinnerung. Darstellung und „Verwendung“ von Zeitzeugen in Ton, Bild und Film. In: BIOS 21 (1), 2008, S. 79-92.

Pohl, Rüdiger: Was ist Gedächtnis/Erinnerung? Das autobiographische Gedächtnis. In: Gudehus, Christian/Eichenberg, Ariane/Welzer, Harald (Hg.): Gedächtnis und Erinnerung. Ein interdisziplinäres Handbuch. Stuttgart/Weimar 2010, S. S. 75-84.

Ratter, Beate/Kruse, Nicole: Klimawandel und Wahrnehmung. Risiko und Risikobewusstsein in Hamburg. In: Böhner, Jürgen/Ratter, Beate (Hg.): Klimawandel und Klimawirkung. Hamburger Symposium Geographie, Band 2. Hamburg 2010, S. 119-137.

Regener, Susanne: Medienamateure - Fotografie und soziale Praxis im Alltag. In: In: Helmut Gold u. a. (Hg.): DIY. Die Mitmach-Revolution. Katalog zur Ausstellung. Frankfurt a. M. 2011, 176-187.

Rheinheimer, Martin: Mythos Sturmflut. Der Kampf gegen das Meer und die Suche nach Identität. In: Demokratische Geschichte. Jahrbuch für Schleswig-Holstein 15, 2003, S. 9-58.

Rieken, Bernd: Nordsee ist Mordsee. Sturmfluten und ihre Bedeutung für die Mentalitätsgeschichte der Friesen. Münster 2005.

Rieken, Bernd: Angst vor dem Meer. Sturmfluten aus Sicht der volkskundlichhistorischen Katastrophenforschung. In: Volkskunde in Rheinland-Pfalz 22 (2007), S. 23-48 (abgekürzt: 2007a). 
Rieken, Bernd: Vom Nutzen volkskundlich-historischer Zugänge für die Katastrophenforschung: New Orleans 2005. In: Hartmann, Andreas/ Meyer, Silke/Mohrmann, Ruth-E. (Hg.): Historizität. Vom Umgang mit Geschichte. Hochschultagung „Historizität als Aufgabe und Perspektive“ der Deutschen Gesellschaft für Volkskunde vom 21.-23. September 2006 in Münster. Münster u. a. 2007, S. 149-162 (abgekürzt: 2007b).

Rieken, Bernd: Schatten über Galtür? Ein Beitrag zur Katastrophenforschung. Münster 2010.

Rieken, Bernd (Hg.): Erzählen über Katastrophen. Beiträge aus deutscher Philologie, Erzählforschung und Psychotherapiewissenschaft. Münster/ New York 2016.

Rieken, Bernd: http://www.sehepunkte.de/2006/09/kommentar/bernd-riekenueber-rezension-die-geschichte-der-flutkatastrophen-35/ (aufgerufen am 11.11.2019)

Risi, Marius: Im Lauf der Zeiten. Oberwalliser Lebenswelten. In: Röösli, Lisa/ Ders.: Lebensbilder - Bilderwelten. Zwei ethnografische Filmprojekte im Alpenraum. Münster 2010, S. 149-254.

Röösli, Lisa/Risi, Marius: Lebensbilder - Bilderwelten. Zwei ethnografische Filmprojekte im Alpenraum. Münster 2010.

Rosenthal, Gabriele/Witte, Nicole: Zur Analyse videographierten Datenmaterials. In: Dies.: Interpretative Sozialforschung. Eine Einführung. 5. aktualisierte und ergänzte Auflage. Weinheim/Basel 2015, S. 130-149.

Ruby, Jay: Picturing Culture. Explorations of Film \& Anthropology. Chicago 2000.

Rüsen, Jörn: Historische Orientierung. Über die Arbeit des Geschichtsbewußtseins, sich in der Zeit zurechtzufinden. Köln/Weimar/Wien 1994.

Rüsen, Jörn: Einleitung. Geschichtsbewußtsein thematisieren - Problemlagen und Analysestrategien. In: Ders. (Hg.): Geschichtsbewusstsein: psychologische Grundlagen, Entwicklungskonzepte, empirische Befunde. Köln/Weimar/ Wien 2001, S. 1-13.

Sabrow, Martin/Frei, Norbert (Hg.): Die Geburt des Zeitzeugen nach 1945. Göttingen 2012.

Saini, Pierrine/Schärer, Thomas: Erinnerung, Film- und Fotoelicitation. In: Bischoff, Christine/Oehme-Jüngling, Karoline/Leimgruber, Walter (Hg.): Methoden der Kulturanthropologie. Bern 2014, S. 313-330.

Schemmer, Janine: Ins Zentrum gerückt. Kultur auf der Elbinsel: Das Bürgerhaus Wilhelmsburg. In: Forschungsstelle für Zeitgeschichte in Hamburg (Hg.): 19 Tage Hamburg. Ereignisse und Entwicklungen der Stadtgeschichte seit den fünfziger Jahren. München/Hamburg 2012, S. 249-262. 
Schlumpf, Hans-Ulrich: Von sprechenden Menschen und Talking Heads. In: Ballhaus, Edmund/Engelbrecht, Beate (Hg.): Der ethnographische Film. Eine Einführung in Methoden und Praxis. Berlin 1995, S.105-119.

Schmidt, Andreas: „Wolken krachen, Berge zittern, und die ganze Erde weint ...“. Zur kulturellen Vermittlung von Naturkatastrophen in Deutschland 1755 bis 1855 . Münster u. a. 1999.

Schmidt-Lauber, Brigitta: Das qualitative Interview oder: Die Kunst des Redenlassens. In: Göttsch, Silke/Lehmann, Albrecht (Hg.): Methoden der Volkskunde. Positionen, Quellen, Arbeitsweisen der Europäischen Ethnologie. Berlin 2001, S. 165-186.

Schmidt-Lauber, Brigitta: Grenzen der Narratologie. Alltagskultur(forschung) jenseits des Erzählens. In: Hengartner, Thomas/Dies. (Hg.): Leben Erzählen. Beiträge zur Erzähl- und Biographieforschung. Festschrift für Albrecht Lehmann. Berlin/Hamburg 2005, S. 145-162.

Schmidt-Lauber, Brigitta: Einleitung zu Panel III: Repräsentationspraxen. Wissens- und Vermittlungsformate kultureller Realitäten. In: Simon, Michael u. a. (Hg.): Bilder. Bücher. Bytes. Zur Medialität des Alltags. 36. Kongress der Deutschen Gesellschaft für Volkskunde in Mainz vom 23. bis 26. September 2007. Münster u. a. 2009, S, 143-147.

Scholz, Tobias: Distanziertes Mitleid. Mediale Bilder, Emotionen und Solidarität angesichts von Katastrophen. Frankfurt a. M./New York 2012.

Schott, Dieter: One City - Three Catastrophes: Hamburg from the Great Fire 1842 to the Great Flood 1962. In: Massard-Guilbaud, Geneviève/Platt, Harold L./Ders. (Hg.): Cities and Catastrophes. Coping with Emergency in European History. Frankfurt a. M. u. a. 2002, S. 185-204.

Schott, Dieter: Naturkatastrophen und städtische Resilienz. Die Hamburger Sturmflut im Kontext städtischer Naturkatastrophen der Neuzeit. In: Heßler, Martina/Kehrt, Christian (Hg.): Die Hamburger Sturmflut von 1962. Risikobewusstsein und Katastrophenschutz aus zeit-, technik- und umweltgeschichtlicher Perspektive. Göttingen/Bristol 2014, S. 37-57.

Schröder, Hans Joachim: Die gestohlenen Jahre. Erzählgeschichten und Geschichtserzählungen im Interview: der Zweite Weltkrieg aus der Sicht ehemaliger Mannschaftssoldaten. Tübingen 1992.

Schröder, Hans Joachim: Topoi des autobiographischen Erzählens. In: Hengartner, Thomas/Schmidt-Lauber, Brigitta (Hg.): Leben - Erzählen. Beiträge zur Erzähl- und Biographieforschung. Festschrift für Albrecht Lehmann. Berlin/Hamburg 2005, S. 17-42. 
Schröder, Hans Joachim: Biografisches Erzählen im Umgang mit Technik. In: Seifert, Manfred/Friedreich, Sönke (Hg.): Alltagsleben biografisch erfassen. Zur Konzeption lebensgeschichtlich orientierter Forschung. Dresden 2009, S. 61-68.

Sedmak, Clemens: Innerlichkeit und Kraft. Studie über epistemische Resilienz. Freiburg i. Br. 2013.

Selke, Stefan: Private Fotos als Bilderrätsel - Eine soziologische Typologie der Sinnhaftigkeit visueller Dokumente im Alltag. In: Ziehe, Irene/Hägele, Ulrich (Hg.): Fotografien vom Alltag - Fotografieren als Alltag. Tagung der Kommission Fotografie der Deutschen Gesellschaft für Volkskunde und der Sektion Geschichte und Archive der Deutschen Gesellschaft für Photographie im Museum Europäischer Kulturen - Staatliche Museen zu Berlin vom 15. bis 17. November 2002. Münster 2004, S. 49-74.

Simon, Michael: Ethnologische Anmerkungen zu Bernd Riekens „Gesprächen mit Einheimischen “. In: Rieken, Bernd (Hg.): Wie bewältigt man das Unfassbare? Interdisziplinäre Zugänge am Beispiel der Lawinenkatastrophe von Galtür. Münster 2015, S. 93-105.

Simonicca, Alessandro: Das Erdbeben von L'Aquila, 6. April 2009. Zwischen Kultur der Katastrophe und politischem Handeln. In: Braun, Karl/ Dieterich, Claus-Marco/Treiber, Angela (Hg.): Materialisierung von Kultur. Diskurse Dinge Praktiken. Würzburg 2015, S. 459-465.

Soell, Hartmut: Helmut Schmidt. 1918-1969. Vernunft und Leidenschaft. München 2003.

Starl, Timm: Knipser. Die Bildgeschichte der privaten Fotografie in Deutschland und Österreich von 1880 bis 1980. München 1995.

Sticher, Brigitta: Das mit dem Unversicherbaren konfrontierte Individuum. Eine psychologische Betrachtung. In: Hempel, Leon/Bartels, Marie/Markwart, Thomas (Hg.): Aufbruch ins Unversicherbare. Zum Katastrophendiskurs der Gegenwart. Bielefeld 2013, S. 307-333.

Sutter, Ove: Erzählte Prekarität. Autobiographische Verhandlungen von Arbeit und Leben im Postfordismus. Frankfurt a. M./New York 2013.

Thießen, Malte: Eingebrannt ins Gedächtnis. Hamburgs Gedenken an Luftkrieg und Kriegsende 1943 bis 2005. Hamburg 2007.

Thießen, Malte: Drei Geschichten des „Feuersturms“. Erinnerungen von Zeitzeugen zwischen privaten, familiären und öffentlichen Erzählungen des Luftkriegs. In: Dierken, Jörg (Hg.): Geisteswissenschaften in der Offensive. Hamburger Standortbestimmungen. Hamburg 2009, S. 353-374. 
Topaloglou, Helena M./Tschugguel, Sabine: Lawinenunglück Galtür. Die Frage nach der individuellen Bewältigung aus personenzentrierter Perspektive. In: Rieken, Bernd (Hg.): Wie bewältigt man das Unfassbare? Interdisziplinäre Zugänge am Beispiel der Lawinenkatastrophe von Galtür. Münster 2015, S. 37-61.

Uekötter, Frank: Wege zu einer ökologischen Erinnerungskultur. In: Ders. (Hg.): Ökologische Erinnerungsorte. Göttingen 2014, S. 7-26.

Viehöver, Willy: Die Klimakatastrophe als ein Mythos der reflexiven Moderne. In: Clausen, Lars/Geenen, Elke M./Macamo, Elisio (Hg.): Entsetzliche soziale Prozesse. Theorie und Empirie der Katastrophen. Münster 2003, S. 247-286.

Volmari, Christian: Vernakulare Fotografie zwischen analogen und digitalen Gebrauchsweisen. Göttingen 2012.

Voss, Martin (Hg.): Der Klimawandel. Sozialwissenschaftliche Perspektiven. Wiesbaden 2010.

Walter, Florian: Kollaborative Feld- und Filmforschung. Gleichberechtigte Formen der Kommunikation und transkulturelle Verstehensprozesse. In: Berliner Blätter 67, 2014, S. 56-67.

Walter, François: Katastrophen. Eine Kulturgeschichte vom 16. bis ins 21. Jahrhundert. Stuttgart 2010.

Welzer, Harald: Das Interview als Artefakt. Zur Kritik an der Zeitzeugenforschung. In: BIOS 13 (1), 2000, S. 51-63.

Welzer, Harald: Das kommunikative Gedächtnis. Eine Theorie der Erinnerung. 2. Auflage. München 2008.

Welzer, Harald: Vom Zeit- zum Zukunftszeugen. Vorschläge zur Modernisierung der Erinnerungskultur. In: Sabrow, Martin/Frei, Norbert (Hg.): Die Geburt des Zeitzeugen nach 1945. Göttingen 2012, S. 33-48.

White, Hayden: Metahistory. Die historische Einbildungskraft im 19. Jahrhundert in Europa. Frankfurt a. M. 1991.

White, Hayden: Metahistory. The Historical Imagination in nineteenth-century Europe. Baltimore/London 1975.

Wilbers, Dörte: „Montierte Erkenntnis“. Überlegungen zur Relevanz der Methoden Eberhard Fechners für den kulturwissenschaftlichen Film. In: Ballhaus, Edmund (Hg.): Kulturwissenschaft, Film und Öffentlichkeit. Münster u. a. 2001, S. 275-289.

Wörner, Simone: Hilfsprogramme - Maßnahmen für die Betroffenen. In: Hötte, Herbert (Hg.): Die große Flut. Katastrophe - Herausforderung - Perspektiven. Begleitbuch zu den Ausstellungen im Hamburger Rathaus und im Museum für Hamburgische Geschichte aus Anlass des 50. Jahrestages der Sturmflut von 1962. Hamburg 2012, S. 58-65. 
Wossidlo, Joachim/Roters, Ulrich: Vorwort. In: Dies. (Hg.): Interview und Film. Volkskundliche und Ethnologische Ansätze zu Methodik und Analyse. Münster u. a. 2003, S. 7-9.

\section{Gedruckte Quellen}

Allgemeine deutsche Real=Encyklopädie für die gebildeten Stände.

(Conversations=Lexikon) Bd. 6. 8. Auflage. Leipzig 1835.

Bericht des vom Senat der Freien und Hansestadt Hamburg berufenen

Sachverständigenausschusses zur Untersuchung des Ablaufs der

Flutkatastrophe. Hamburg 1962.

Boie, Kirsten: Ringel, Rangel, Rosen. Hamburg 2010.

Boie-Eyer, Ursula: Die Hamburger Sturmflut. Göttingen o. J. (ca. 1963).

Eismann, Martina/Mierach, Michael: Wenn die Flut kommt ... Erinnerungen an die Katastrophe von 1962 und heutiger Hochwasserschutz. Hg. von der Behörde für Bau und Verkehr Hamburg/Amt für Wasserwirtschaft. Hamburg o. J. (2002).

Falke, Ulla: „Bis zur großen Sturmflut war mir nicht bewusst, dass ich auf einer Insel lebe." In: Geschichtswerkstatt Wilhelmsburg \& Hafen (Hg.): Wilhelmines Gedächtnis. Geschichte weiblich in fünf exemplarischen Lebensläufen. Hamburg 2002, S. 17-39.

Fechner, Eberhard: Rede anlässlich der PEN-Tagung Erlangen 1978. In: Nagel, Josef/Kirschner, Klaus (Hg.): Eberhard Fechner. Die Filme, gesammelte Aufsätze und Materialien. Erlangen 1984. S. 183-197.

Geschichtswerkstatt Wilhelmsburg/Pauw Literaturmanagement: Als der Deich brach. Sturmflut - Wortflut. Jugendliche erzählen. Hamburg 2012.

Die große Flut in Hamburg. Eine Chronik der Katastrophe vom Februar 1962. Im Auftrag und in Zusammenarbeit mit der Schulbehörde zusammengestellt von Hans Bütow. Hamburg o. J. (1963).

Hamburger Abendblatt: Das war die große Flut. Chronik und Dokumentation der bitteren Tage im Februar 1962. Sonderdruck. Hamburg 1962.

Herlin, Hans: Die Sturmflut. Nordseeküste und Hamburg im Februar 1962. Hamburg 1982 (Hg. zusammen mit dem Hamburger Abendblatt).

Herlin, Hans: Die Sturmflut. Nordseeküste und Hamburg im Februar 1962. Hamburg 1997 (Wiederauflage mit stark reduzierter Anzahl von Fotografien).

Hötte, Herbert (Hg.): Die große Flut. Katastrophe. Herausforderung. Perspektiven. Begleitbuch zu den Ausstellungen im Hamburger Rathaus und im Museum für Hamburgische Geschichte aus Anlass des 50. Jahrestages der Sturmflut vom Februar 1962. Hamburg 2012. 
Homfeld, Karl: Die große Flut von 1962. Zerstörung und Wiederaufbau in Wilhelmsburg. Hamburg 1982.

Institut für angewandte Sozialwissenschaft: Die Flut. Ergebnisse einer Repräsentativerhebung in Hamburg. Winter 1962/63. Bad Godesberg 1964.

Keesenberg, Hermann: Die Wilhelmsburger Sturmflutkatastrophe 1962 aus heimatgeschichtlicher Sicht. Hg. von der Wilhelmsburger Zeitung. Hamburg o. J. (ca. 1962).

Ley, Raymond (Hg.): Die Nacht der großen Flut. Gespräche mit Zeitzeugen und Helmut Schmidt. Hamburg 2006.

Markert, Margret/Paech, Frauke: 50 Jahre Flut. Projektbericht über die Veranstaltungsreihe zum 50sten Gedenkjahr an die Hamburger Sturmflut von 1962 in Wilhelmsburg. Geschichtswerkstatt Wilhelmsburg \& Hafen. Hamburg 2012.

Mohr, Henry/Köpke, Rudolf (Hg.): Die große Flut. Augenzeugen berichten über ihre Erlebnisse vom 16. auf den 17. Februar 1962. Hamburg 2003.

Parnack, Charlotte/Kuhr, Daniela: Angriff eines echten Rüpels. Wie sich Hamburg auf „Xaver" vorbereitet - und mit welchen Problemen auf Schienen und Straßen zu rechnen ist. In: Süddeutsche Zeitung vom 5.12.2013, S. 2.

Schuller, Alexander: Sturmflut über Hamburg. Die Nacht, in der die Stadt ertrank. Ein Tatsachenroman. München 2006.

Sturmflut-Katastrophe Februar 1962. Stade/Buxtehude 1962.

Wilhelmsburg 1962. Hg. von den Wilhelmsburger Heimatvereinen. Hamburg 1962.

Wilhelmsburger Zeitung (Hg.): Der 17. Februar 1962. Hamburg o. J. (ca. 1962). „Wir haben viel Glück gehabt“. Lebensgeschichten zur Sturmflut 1962. Hg. von der Stadtteilschule Stellingen/Ida Ehre Schule. Hamburg 2016 (inkl. DVD mit einem Interview mit Helmut Schmidt).

\section{Foto- und Filmquellen}

Dierks, Jutta

Flutkatastrophe in Hamburg (1962), Landesbildstelle Hamburg

Die große Flut (1962), Baubehörde Hamburg - Tiefbauamt

Geschichtswerkstatt Wilhelmsburg \& Hafen

Heimatmuseum Wilhelmsburg e. V.

Kulturkreis Finkenwerder e. V.

Stanik, Horst

Wendt, Gerhardt 


\section{Anhang}

\section{Interviewsample - Biografische Informationen}

Im Film verwendete Interviews

\begin{tabular}{|c|c|c|c|}
\hline Name & $\begin{array}{l}\text { Geb.- } \\
\text { Jahr }\end{array}$ & 1962: & $\begin{array}{l}\text { Datum des } \\
\text { Interviews }\end{array}$ \\
\hline Bode, Claus & 1952 & $\begin{array}{l}9 \text { Jahre, Schüler, Sohn der Inhaber der } \\
\text { „Gaststätte Sohre“; Wilhelmsburg, } \\
\text { Ortsteil Kirchdorf }\end{array}$ & 15.11 .2004 \\
\hline Dierks, Jutta & 1943 & $\begin{array}{l}18 \text { Jahre, Angestellte, Deutsche Werft, } \\
\text { Finkenwerder; Francop; Schwester von } \\
\text { Bernd Meyer u. Gitta Rosenau }\end{array}$ & 27.08.2004 \\
\hline Felka, Gerd & $\begin{array}{l}\text { ca. } \\
1937\end{array}$ & $\begin{array}{l}\text { ca. } 25 \text { Jahre, verheiratet, } 1 \text { Kind, } \\
\text { Arbeiter im Baugewerbe; } \\
\text { Wilhelmsburg, Ortsteil Georgswerder } \\
\text { (KGV Brummerkaten) }\end{array}$ & 20.10 .2004 \\
\hline Fick, Adolf & 1950 & 11 Jahre, Schüler; Finkenwerder & 10.11 .2004 \\
\hline $\begin{array}{l}\text { Göhrke, Hans- } \\
\text { Peter }\end{array}$ & 1941 & $\begin{array}{l}21 \text { Jahre, Wehrdienstleistender; } \\
\text { Wilhelmsburg, Ortsteil Reiherstieg }\end{array}$ & 12.10 .2004 \\
\hline $\begin{array}{l}\text { Hofmann, } \\
\text { Hans-Heinrich }\end{array}$ & 1943 & $\begin{array}{l}18 \text { Jahre, Schüler; Wilhelmsburg, } \\
\text { Ortsteil Kirchdorf }\end{array}$ & 15.11 .2004 \\
\hline Kannen, Ingrid & 1942 & $\begin{array}{l}20 \text { Jahre, verheiratet, Friseurin; } \\
\text { Wilhelmsburg, Ortsteil Kirchdorf }\end{array}$ & 15.11 .2004 \\
\hline Krause, Brigitte & 1948 & $\begin{array}{l}14 \text { Jahre, Schülerin; Wilhelmsburg, } \\
\text { Ortsteil Georgswerder (KGV } \\
\text { Brummerkaten); Schwester von Rena- } \\
\text { te Heymann u. Helga Rostowski }\end{array}$ & 12.10 .2004 \\
\hline Kröger, Adolf & 1924 & $\begin{array}{l}38 \text { Jahre, verheiratet, } 2 \text { Kinder, } \\
\text { Beamter bei der Oberfinanzdirektion } \\
\text { Hamburg; Finkenwerder }\end{array}$ & 10.11 .2004 \\
\hline Meyer, Bernd & 1949 & $\begin{array}{l}13 \text { Jahre, Schüler; Francop; Bruder } \\
\text { von Jutta Dierks u. Gitta Rosenau }\end{array}$ & 27.08.2004 \\
\hline Meyer, Ernst & 1935 & $\begin{array}{l}27 \text { Jahre, ledig, Bäckermeister; } \\
\text { Wilhelmsburg, Ortsteil Georgswerder }\end{array}$ & 15.11 .2004 \\
\hline
\end{tabular}




\begin{tabular}{|c|c|c|c|}
\hline Name & $\begin{array}{l}\text { Geb.- } \\
\text { Jahr }\end{array}$ & 1962: & $\begin{array}{l}\text { Datum des } \\
\text { Interviews }\end{array}$ \\
\hline Rosenau, Gitta & 1953 & $\begin{array}{l}8 \text { Jahre, Schülerin; Francop; Schwester } \\
\text { von Jutta Dierks u. Bernd Meyer }\end{array}$ & 27.08.2004 \\
\hline $\begin{array}{l}\text { Rostowski, } \\
\text { Helga }\end{array}$ & 1950 & $\begin{array}{l}12 \text { Jahre, Schülerin; Wilhelmsburg, } \\
\text { Ortsteil Georgswerder (KGV Brum- } \\
\text { merkaten); Schwester von Renate } \\
\text { Heymann u. Brigitte Krause }\end{array}$ & 29.09 .2004 \\
\hline Schaub, Maren & 1947 & $\begin{array}{l}14 \text { Jahre, Schülerin; Neuenfelde, Orts- } \\
\text { teil Rosengarten }\end{array}$ & 09.11 .2004 \\
\hline $\begin{array}{l}\text { Schwartau, } \\
\text { Elisabeth }\end{array}$ & 1931 & $\begin{array}{l}31 \text { Jahre, verheiratet, } 3 \text { Kinder, Mit- } \\
\text { arbeit im Gemüsebaubetrieb der } \\
\text { Familie; Altenwerder }\end{array}$ & 05.11 .2004 \\
\hline Seemann, Ursula & $\begin{array}{l}\text { ca. } \\
1937\end{array}$ & $\begin{array}{l}\text { ca. } 25 \text { Jahre, verheiratet, } 1 \text { Kind, Ver- } \\
\text { käuferin im Lebensmitteleinzelhandel; } \\
\text { Wilhelmsburg, Ortsteil Reiherstieg }\end{array}$ & 17.11.2004 \\
\hline Wendt, Gerhard & 1938 & $\begin{array}{l}24 \text { Jahre, verheiratet, selbstständig } \\
\text { Gemüsebaubetrieb u. Blumengeschäft; } \\
\text { Wilhelmsburg, Ortsteil Kirchdorf }\end{array}$ & 20.10 .2004 \\
\hline Wenzel, Herbert & 1929 & $\begin{array}{l}33 \text { Jahre, verheiratet mit Marlies } \\
\text { Wenzel, } 2 \text { Kinder, Arbeitstechniker } \\
\text { Howaldtswerke Neuhof; } \\
\text { Wilhelmsburg, Ortsteil Kirchdorf }\end{array}$ & 29.09 .2004 \\
\hline Wenzel, Marlies & $\begin{array}{l}\text { ca. } \\
1935\end{array}$ & $\begin{array}{l}\text { ca. } 27 \text { Jahre, verheiratet mit Herbert } \\
\text { Wenzel, } 2 \text { Kinder, Hausfrau; } \\
\text { Wilhelmsburg, Ortsteil Kirchdorf }\end{array}$ & 29.09 .2004 \\
\hline
\end{tabular}

Weitere gefilmte Interviews

\begin{tabular}{|l|l|l|l|}
\hline Name & $\begin{array}{l}\text { Geb.- } \\
\text { Jahr }\end{array}$ & $\mathbf{1 9 6 2 :}$ & $\begin{array}{l}\text { Datum des } \\
\text { Interviews }\end{array}$ \\
\hline Ahrens, Jürgen & 1933 & $\begin{array}{l}\text { 29 Jahre, ledig; Wilhelmsburg, Orts- } \\
\text { teil Kirchdorf }\end{array}$ & 12.10 .2004 \\
\hline $\begin{array}{l}\text { Beckedorf, } \\
\text { Heinz }\end{array}$ & 1937 & $\begin{array}{l}\text { 24 Jahre, verlobt, Meister in einem } \\
\text { Werftbetrieb auf Neuhof; Altenwerder }\end{array}$ & 19.10 .2004 \\
\hline Fock, Uwe & 1941 & $\begin{array}{l}\text { 21 Jahre, Wehrdienstleistender; } \\
\text { Finkenwerder }\end{array}$ & 09.11 .2004 \\
\hline Hilke, Fritz & 1912 & $\begin{array}{l}\text { 49 Jahre, verheiratet, 2 Kinder, Polizei- } \\
\text { meister (Einzelposten); Neuenfelde }\end{array}$ & 14.10 .2004 \\
\hline
\end{tabular}




\begin{tabular}{|c|c|c|c|}
\hline Name & $\begin{array}{l}\text { Geb.- } \\
\text { Jahr }\end{array}$ & 1962: & $\begin{array}{l}\text { Datum des } \\
\text { Interviews }\end{array}$ \\
\hline $\begin{array}{l}\text { Hückmann, } \\
\text { Renate }\end{array}$ & 1939 & $\begin{array}{l}23 \text { Jahre, ledig, Angestellte in einem } \\
\text { Baugeschäft; Wilhelmsburg, Ortsteil } \\
\text { Reiherstieg }\end{array}$ & 03.08 .2004 \\
\hline Meyer, Elsbeth & 1918 & $\begin{array}{l}43 \text { Jahre, verheiratet, } 3 \text { Kinder, Haus- } \\
\text { frau; Francop; Mutter von Jutta } \\
\text { Dierks, Bernd Meyer u. Gitta Rosenau }\end{array}$ & 27.08.2004 \\
\hline $\begin{array}{l}\text { Schürmann, } \\
\text { Anni }\end{array}$ & 1914 & $\begin{array}{l}38 \text { Jahre, verheiratet, } 1 \text { Kind, Hausfrau, } \\
\text { Teilzeitbeschäftigung in einer Stickerei; } \\
\text { Wilhelmsburg, Ortsteil Georgswerder; } \\
\text { Tante von Renate Heymann, Brigitte } \\
\text { Krause u. Helga Rostowski }\end{array}$ & 20.10 .2004 \\
\hline $\begin{array}{l}\text { Seufert, } \\
\text { Sieglinde }\end{array}$ & 1938 & $\begin{array}{l}24 \text { Jahre, ledig, Krankenschwester } \\
\text { Krankenhaus Groß Sand; Wilhelms- } \\
\text { burg, Ortsteil Reiherstieg }\end{array}$ & 13.11 .2004 \\
\hline Seufert, Werner & $\begin{array}{l}\text { ca. } \\
1936\end{array}$ & $\begin{array}{l}\text { ca. } 26 \text { Jahre, ledig, Tiefdrucker; } \\
\text { Bahrenfeld; Ehemann von Sieglinde } \\
\text { Seufert }\end{array}$ & 13.11 .2004 \\
\hline Spinna, Heinz & $\begin{array}{l}\text { ca. } \\
1935\end{array}$ & $\begin{array}{l}\text { ca. } 27 \text { Jahre, verheiratet, } 1 \text { Kind, } \\
\text { Maschinenbauer; Wilhelmsburg, Orts- } \\
\text { teil Kirchdorf }\end{array}$ & 13.10 .2004 \\
\hline Stanik, Horst & $\begin{array}{l}\text { ca. } \\
1928\end{array}$ & $\begin{array}{l}\text { ca. } 35 \text { Jahre, verheiratet, } 1 \text { Kind, } \\
\text { Techniker Raffinerie Hohe Schaar; } \\
\text { Wilhelmsburg }\end{array}$ & 03.08.2004 \\
\hline Wagner, Kurt & $\begin{array}{l}\text { ca. } \\
1935\end{array}$ & $\begin{array}{l}\text { ca. } 27 \text { Jahre, verheiratet, } 1 \text { Kind, } \\
\text { Maschinenbauer; Finkenwerder } \\
\text { 2004: Vorsitzender des Kulturkreises } \\
\text { Finkenwerder e. V. }\end{array}$ & 09.11 .2004 \\
\hline
\end{tabular}

\section{Audio-Interviews}

\begin{tabular}{|l|l|l|l|}
\hline Name & $\begin{array}{l}\text { Geb.- } \\
\text { Jahr }\end{array}$ & 1962: & $\begin{array}{l}\text { Datum des } \\
\text { Interviews }\end{array}$ \\
\hline Buttgereit, Herta & $\begin{array}{l}\text { keine } \\
\text { Angabe }\end{array}$ & $\begin{array}{l}\text { verheiratet, vermutlich Hausfrau; } \\
\text { Wilhelmsburg, Ortsteil Kirchdorf }\end{array}$ & $\begin{array}{l}15.09 .2004 \\
\text { (zus. m. Christa } \\
\text { Moldenhauer) }\end{array}$ \\
\hline Deckert, Kurt & 1920 & $\begin{array}{l}\text { 41 Jahre, verheiratet, Kaufmann u. } \\
\text { Gaststättenbesitzer „Sächsischer Hof“; } \\
\text { Finkenwerder }\end{array}$ & $\begin{array}{l}14.07 .2004 \\
\text { (zus. m. Mar- } \\
\text { gret Kolzer) }\end{array}$ \\
\hline
\end{tabular}




\begin{tabular}{|c|c|c|c|}
\hline Name & $\begin{array}{l}\text { Geb.- } \\
\text { Jahr }\end{array}$ & 1962: & $\begin{array}{l}\text { Datum des } \\
\text { Interviews }\end{array}$ \\
\hline Dierks, Emma & $\begin{array}{l}\text { keine } \\
\text { Angabe }\end{array}$ & $\begin{array}{l}\text { verheiratet, } 3 \text { Kinder, Hausfrau u. } \\
\text { Mitarbeit im landwirtschaftlichen } \\
\text { Betrieb; Neuenfelde }\end{array}$ & $\begin{array}{l}13.09 .2004 \\
\text { (zus. m. } \\
\text { Günter u. Nor- } \\
\text { bert Dierks) }\end{array}$ \\
\hline Dierks, Jutta & 1943 & $\begin{array}{l}18 \text { Jahre, Angestellte, Deutsche Werft, } \\
\text { Finkenwerder; Francop; Schwester von } \\
\text { Bernd Meyer und Gitta Rosenau }\end{array}$ & 06.08 .2004 \\
\hline $\begin{array}{l}\text { Eggers, } \\
\text { Margarete }\end{array}$ & $\begin{array}{l}\text { keine } \\
\text { Angabe }\end{array}$ & $\begin{array}{l}\text { verheiratet, vermutlich Hausfrau; } \\
\text { Wilhelmsburg, Ortsteil Reiherstieg }\end{array}$ & 16.04 .2004 \\
\hline Falke, Ulla & 1937 & $\begin{array}{l}25 \text { Jahre, ledig, Kinderpflegerin; } \\
\text { Wilhelmsburg, Ortsteil Kirchdorf }\end{array}$ & $\begin{array}{l}09.10 .2003 \text { u. } \\
10.02 .2004\end{array}$ \\
\hline Frick, Lisa & $\begin{array}{l}\text { keine } \\
\text { Angabe }\end{array}$ & $\begin{array}{l}\text { verheiratet, } 1 \text { Kind, Hausfrau u. Pfarr- } \\
\text { sekretärin Kreuzkirche; Wilhelmsburg, } \\
\text { Ortsteil Kirchdorf }\end{array}$ & 24.09 .2004 \\
\hline Gräfe, Else & 1917 & $\begin{array}{l}44 \text { Jahre, verheiratet, vermutlich } \\
\text { Hausfrau, } 1 \text { Kind; Wilhelmsburg }\end{array}$ & 07.05 .2004 \\
\hline Henke, Christa & 1936 & $\begin{array}{l}26 \text { Jahre, ledig, Büroangestellte; } \\
\text { Wilhelmsburg, Ortsteil Reiherstieg }\end{array}$ & 08.11 .2004 \\
\hline $\begin{array}{l}\text { Heymann, } \\
\text { Renate }\end{array}$ & 1953 & $\begin{array}{l}9 \text { Jahre, Schülerin; Wilhelmsburg, } \\
\text { Ortsteil Georgswerder (KGV Brum- } \\
\text { merkaten); Schwester von Brigitte } \\
\text { Krause und Helga Rostowski }\end{array}$ & 16.06 .2004 \\
\hline $\begin{array}{l}\text { Hofmann, } \\
\text { Hans-Heinrich }\end{array}$ & 1943 & $\begin{array}{l}18 \text { Jahre, Schüler; Wilhelmsburg, } \\
\text { Ortsteil Kirchdorf }\end{array}$ & 16.06 .2004 \\
\hline Hundeck, Astrid & 1922 & $\begin{array}{l}39 \text { Jahre, verheiratet, Technische } \\
\text { Zeichnerin u. Fotografin; Wilhelms- } \\
\text { burg, Ortsteil Kirchdorf }\end{array}$ & 14.05 .2004 \\
\hline $\begin{array}{l}\text { Kaptuller- } \\
\text { Eichberg, Bärbel }\end{array}$ & 1943 & $\begin{array}{l}19 \text { Jahre, Ausbildung zur Erzieherin; } \\
\text { Tochter des damaligen Ortsamtsleiters } \\
\text { Hermann Westphal; Wilhelmsburg, } \\
\text { Ortsteil Reiherstieg }\end{array}$ & 27.07 .2004 \\
\hline Kolzer, Margret & 1942 & $\begin{array}{l}19 \text { Jahre, Zahnarzthelferin; } \\
\text { Finkenwerder }\end{array}$ & $\begin{array}{l}14.07 .2004 \\
\text { (zus. m. Kurt } \\
\text { Deckert) }\end{array}$ \\
\hline Marotzki, Inge & 1926 & $\begin{array}{l}36 \text { Jahre, verheiratet, } 2 \text { Kinder, ver- } \\
\text { mutlich Hausfrau; Wilhelmsburg, } \\
\text { Ortsteil Reiherstieg }\end{array}$ & 30.04 .2004 \\
\hline
\end{tabular}




\begin{tabular}{|c|c|c|c|}
\hline Name & $\begin{array}{l}\text { Geb.- } \\
\text { Jahr }\end{array}$ & 1962: & $\begin{array}{l}\text { Datum des } \\
\text { Interviews }\end{array}$ \\
\hline $\begin{array}{l}\text { Moldenhauer, } \\
\text { Christa }\end{array}$ & 1933 & $\begin{array}{l}28 \text { Jahre, verheiratet, vermutlich } \\
\text { Hausfrau; Wilhelmsburg, Ortsteil } \\
\text { Kirchdorf }\end{array}$ & $\begin{array}{l}15.09 .2004 \\
\text { (zus. m. Herta } \\
\text { Butgereit) }\end{array}$ \\
\hline Quast, Grete & $\begin{array}{l}\text { keine } \\
\text { Angabe }\end{array}$ & $\begin{array}{l}\text { verheiratet, } 3 \text { Kinder, Hausfrau, Mit- } \\
\text { arbeit im eigenen landwirtschaftlichen } \\
\text { Betrieb; Neuenfelde }\end{array}$ & 20.10 .2004 \\
\hline Pein, Christel & 1927 & $\begin{array}{l}34 \text { Jahre, verheiratet, } 1 \text { Kind, Schicht- } \\
\text { arbeiterin in einem Furnierwerk; } \\
\text { Wilhelmsburg, Ortsteil Georgswerder } \\
\text { (KGV Brummerkaten) }\end{array}$ & $\begin{array}{l}03.12 .2004 \\
\text { (zus. m. } \\
\text { der Tochter } \\
\text { Waltraud } \\
\text { Schmoldt) }\end{array}$ \\
\hline Röttmer, Reimar & 1948 & $\begin{array}{l}23 \text { Jahre, ledig, Facharbeiter im } \\
\text { Freihafen; Wilhelmsburg, Ortsteil } \\
\text { Reiherstieg }\end{array}$ & $\begin{array}{l}25.10 .2004 \\
\text { (zus. m. } \\
\text { Ehefrau Renate } \\
\text { Röttmer) }\end{array}$ \\
\hline Röttmer, Renate & 1950 & $\begin{array}{l}21 \text { Jahre, ledig, Postangestellte; } \\
\text { Wilhelmsburg, Ortsteil Reiherstieg }\end{array}$ & $\begin{array}{l}25.10 .2004 \\
\text { (zus. m. Ehe- } \\
\text { mann Reimar } \\
\text { Röttmer) }\end{array}$ \\
\hline $\begin{array}{l}\text { Schulz, } \\
\text { Edeltraut }\end{array}$ & 1942 & $\begin{array}{l}19 \text { Jahre, Postangestellte; } \\
\text { Wilhelmsburg, Ortsteil Reiherstieg }\end{array}$ & 30.04 .2004 \\
\hline Weidner, Holger & 1945 & $\begin{array}{l}16 \text { Jahre, Schüler; Rothenburgsort } \\
\text { (KGV Rothenburgsort) }\end{array}$ & 21.09 .2004 \\
\hline Werner, Harald & $\begin{array}{l}\text { keine } \\
\text { Angabe }\end{array}$ & $\begin{array}{l}\text { verheiratet, } 1 \text { Kind, Wehrdienstleis- } \\
\text { tender; Waltershof }\end{array}$ & $\begin{array}{l}11.11 .2004 \\
\text { (zus. m. } \\
\text { Ehefrau Karin } \\
\text { Werner) }\end{array}$ \\
\hline Werner, Karin & $\begin{array}{l}\text { keine } \\
\text { Angabe }\end{array}$ & $\begin{array}{l}\text { verheiratet, } 1 \text { Kind, Hausfrau u. Teil- } \\
\text { zeitbeschäftigung im Friseurbedarfs- } \\
\text { handel; Waltershof }\end{array}$ & $\begin{array}{l}11.11 .2004 \\
\text { (zus. m. Ehe- } \\
\text { mann Harald } \\
\text { Werner) }\end{array}$ \\
\hline Zirwes, Olga & $\begin{array}{l}\text { keine } \\
\text { Angabe }\end{array}$ & $\begin{array}{l}\text { verheiratet, } 1 \text { Kind, Mitarbeit im } \\
\text { Garagenbetrieb ihres Ehemannes; } \\
\text { Wilhelmsburg, Ortsteil Reiherstieg }\end{array}$ & 19.03 .2004 \\
\hline
\end{tabular}


D ie Sturmflut von 1962 ist tief im kollektiven Gedächtnis der Stadt Hamburg verankert und die Ereignisse werden deutschlandweit erinnert. Damals starben mehr als 300 Menschen, unzählige Nutz- und Haustiere ertranken, Landwirtschaftsbetriebe, Wohn- und Geschäftshäuser wurden zerstört. Tausende Einwohner_innen der damals überfluteten Stadtteile aber haben dieses Ereignis überlebt, und viele von ihnen können noch heute davon erzählen. Von großer Bedeutung sind diese Erinnerungserzählungen, da sie den Betroffenen nach der Überwindung von Chaos und Verlusterfahrungen auch zur Herstellung von innerer Ordnung verhalfen.

Die Analysen der über fünfzig Interviews verdeutlichen darüber hinaus, dass die Befragten ein profundes Erfahrungswissen eint, das - in Hinblick auf mögliche zukünftige Gefahren durch Sturmfluten beziehungsweise Hochwasser - auch eine Form von Risikobewusstsein darstellt. Somit sind die Erinnerungserzählungen Bestandteil von Cultures of Awareness.

Die Ergebnisse dieser Untersuchung, welche die Methoden der volkskundlichkulturanthropologischen Erzählforschung mit denen des ethnografischen Films verbindet, sind daher ebenfalls ein Beitrag zur aktuellen transdisziplinären Erforschung von Resilienz und Vulnerabilität.

Der hier online zugänglich gemachte Dokumentarfilm Flut 1962 - Erinnern. Gedenken. Erzählen. fasst die wesentlichen Befunde der Studie zusammen und überführt sie in Bild und Ton. 